\title{
INNOVATIVE SEPARATIONS RESEARCH AND DEVELOPMENT NEEDS FOR Advanced Fuel Cycles
}

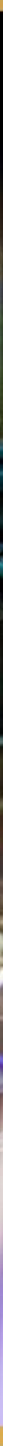

Science-Based Technology Directions for Accelerating Nuclear Fuel Recycle 


\begin{abstract}
This report was prepared as an account of work sponsored by an agency of the United States Government. Neither the United States Government nor any agency thereof, nor any of their employees, makes any warranty, express or implied, or assumes any legal liability or responsibility for the accuracy, completeness, or usefulness of any information, apparatus, product, or process disclosed, or represents that its use would not infringe privately owned rights. Reference herein to any specific commercial product, process, or service by trade name, trademark, manufacturer, or otherwise, does not necessarily constitute or imply its endorsement, recommendation, or favoring by the United States Government or any agency thereof. The views and opinions of authors expressed herein do not necessarily state or reflect those of the United States Government or any agency thereof.
\end{abstract}

Suggested citation: Innovative Separations R\&D Needs for Advanced Fuel Cycles Workshop: Science-Based Technology Directions for Accelerating Nuclear Fuel Recycle; Final workshop report to the US Department of Energy, Office of Nuclear Energy: Washington DC, 2022.

On the cover, Amanda Casella, chemical engineer at Pacific Northwest National Laboratory, adjusts a bank of $2 \mathrm{~cm}$ centrifugal contactors in a glovebox. The contactors have been used for testing flowsheets of proposed processes for recovering actinides from dissolved used nuclear fuel. Contactors of this size provide a realistic demonstration of multistage countercurrent solvent extraction in a laboratory setting. They can be adapted for use in hot cells to test high-activity solutions, and the centrifugal contactor technology can be readily scaled up for use in plant-scale processes. 


\section{Report for the US Department of Energy, Office of Nuclear Energy \\ Innovative Separations R\&D Needs for Advanced Fuel Cycles Workshop}

August 30-September 1, 2021

Hosted virtually by Oak Ridge National Laboratory

Workshop Co-Chairs

Bruce A. Moyer

Oak Ridge National Laboratory

Gregg J. Lumetta

Pacific Northwest National Laboratory

Panel Leads

Solid-Gas Separations

Stephanie H. Bruffey

Oak Ridge National Laboratory

Sarah Finkeldei

University of California, Irvine

\section{Solid-Liquid Separations}

Ken C. Marsden

Michael F. Simpson

Idaho National Laboratory

University of Utah

\section{Liquid-Liquid Separations}

Mark P. Jensen

Colorado School of Mines

Peter R. Zalupski

Idaho National Laboratory

Crosscutting R\&D Leads

Aurora E. Clark

Washington State University

Ping Yang

Los Alamos National Laboratory

Gregory P. Horne

Idaho National Laboratory

\section{Office of Nuclear Energy Lead}

Stephen Kung, Director, Office of Materials and Chemical Technologies 


\section{TABLE OF CONTENTS}

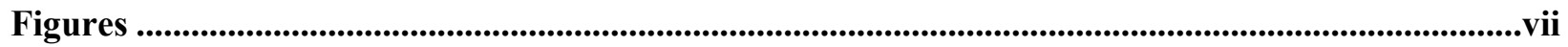

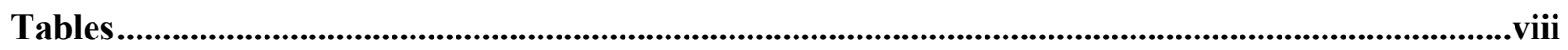

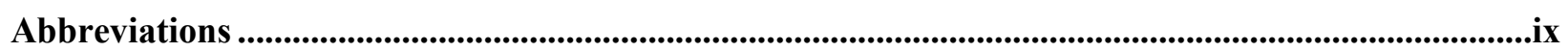

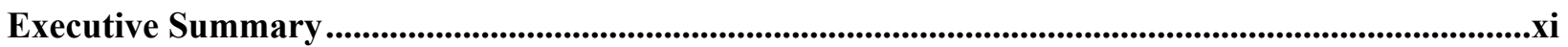

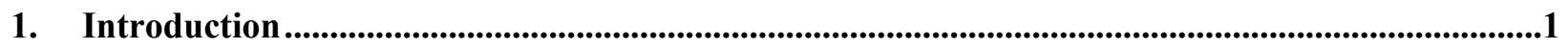

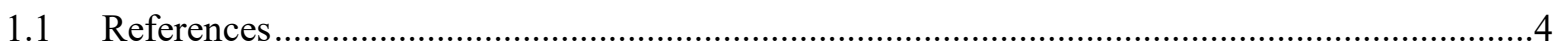

2. FRD 1: Develop Methods to Efficiently Remove and Treat Coating and Cladding

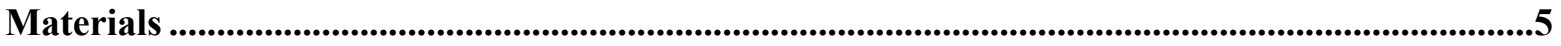

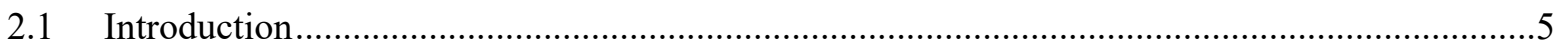

2.2 Research Needs for Decladding and Decontamination ........................................................6

2.3 Removal of Coating Materials from TRISO-Based Fuels ......................................................

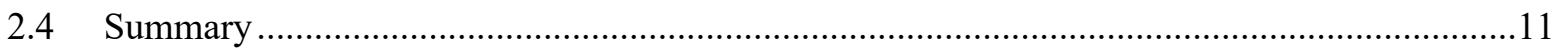

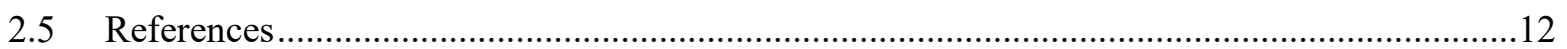

3. FRD 2: Devise Chemistry that Operates Directly on Irradiated Fuel .............................................14

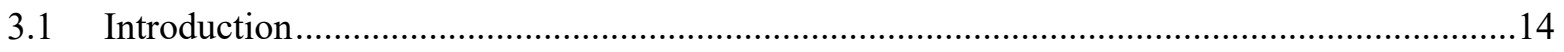

3.2 Voloxidation and Direct Dissolution in Process Solvents .....................................................15

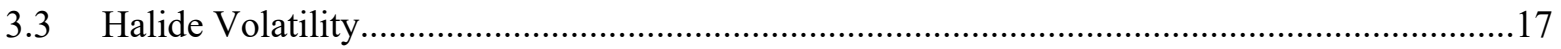

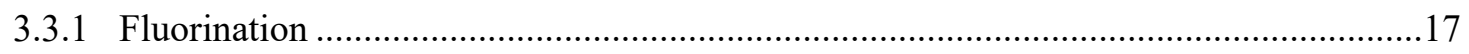

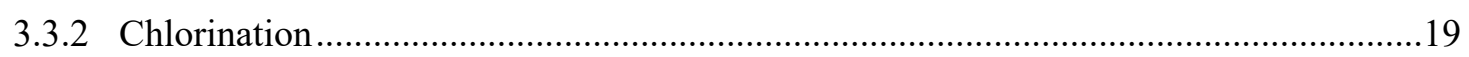

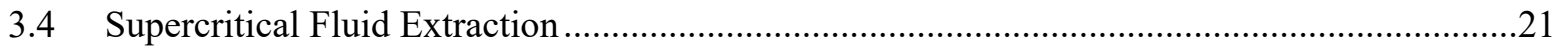

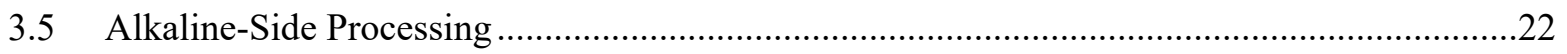

3.6 Separations for Molten Salt Reactor Fuel Salts ...................................................................24

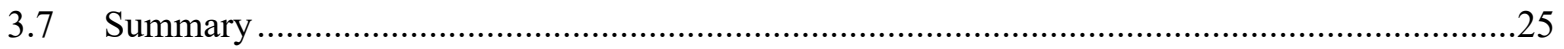

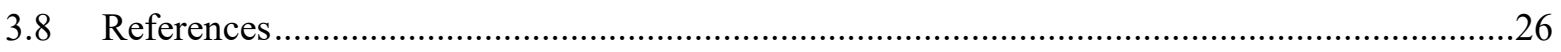

4. FRD 3: Design Robust Materials for Separation of Gas-Phase Species ......................................29

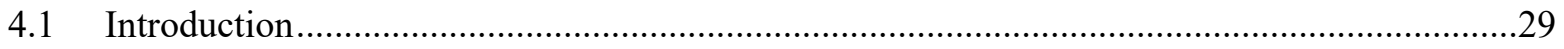

4.2 Technical Challenges in Materials Development for Gas-Solid Nuclear Fuel Cycle

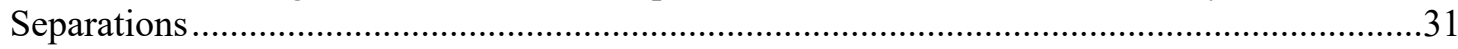

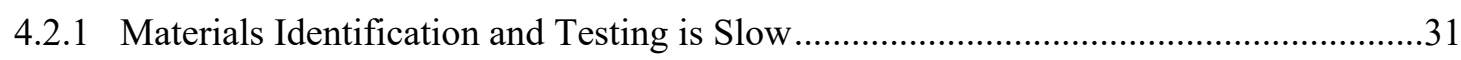

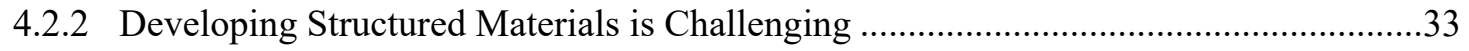

4.2.3 Working Capacity and Slow Adsorption Kinetics are Obstacles to

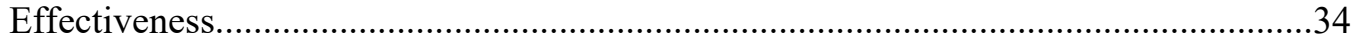

4.2.4 Materials Development should Consider Final Disposition........................................36

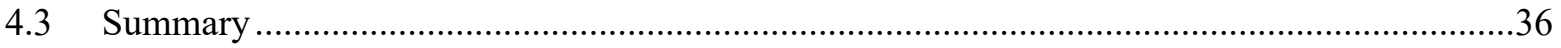

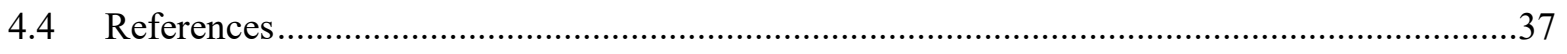

5. FRD 4: Apply Physical Phenomena, Fields, and Gradients to Intensify Separations ..................39

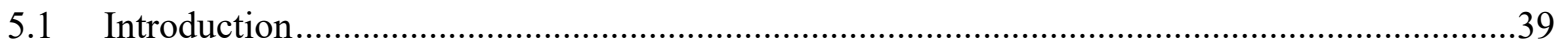


5.2 Harnessing External Forces and Fields for Separations ..................................................... 40

5.3 Controlling Interfacial Chemistry to Drive Separations ........................................................43

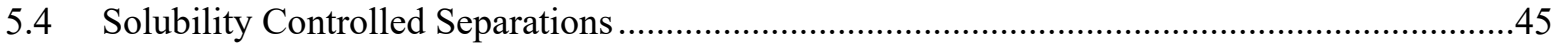

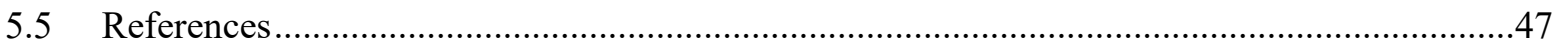

6. FRD 5: Exploit Principles of Coordination Chemistry to Simplify Actinide Separations..........49

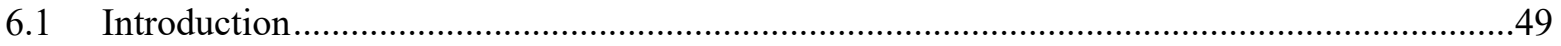

6.2 Current State of Coordination Chemistry in Actinide Separations .........................................50

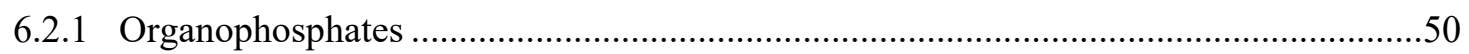

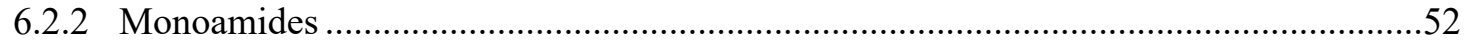

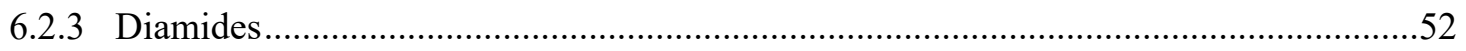

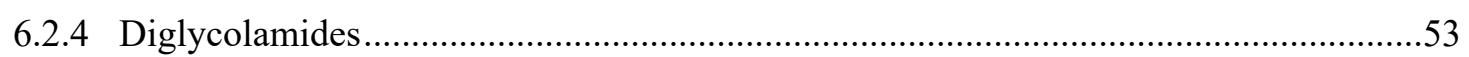

6.2.5 Bis-triazinyl-Pyridines, Bis-Triazinyl-Bipyridines and Bis-Triazolylpyridines ............53

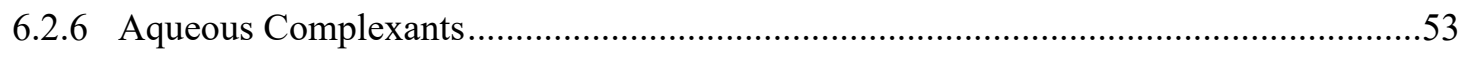

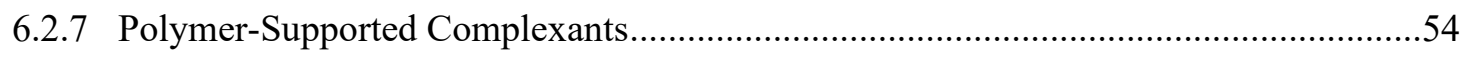

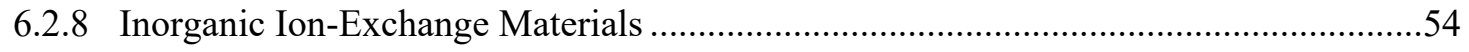

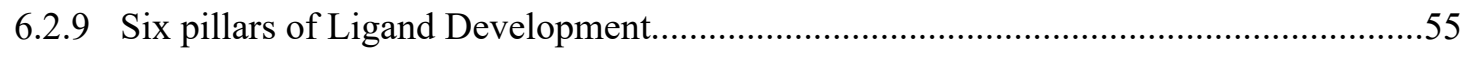

6.3 Research Needs to Advance the Application of Coordination Chemistry ................................55

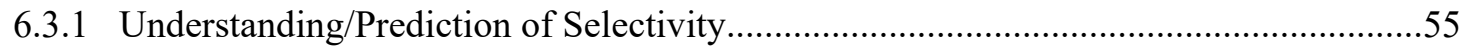

6.3.2 Accelerate Ligand/Adsorbent Design with Integrated High-Throughput Synthesis, Experimental Evaluation, High-Performance Computing, and Data

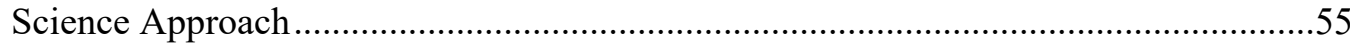

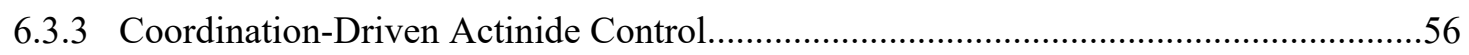

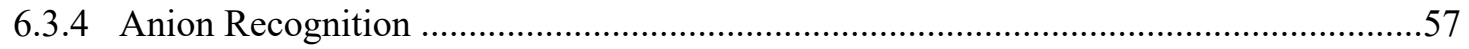

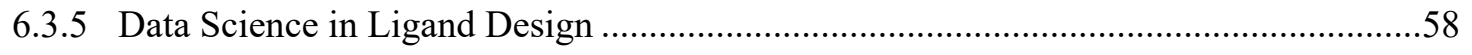

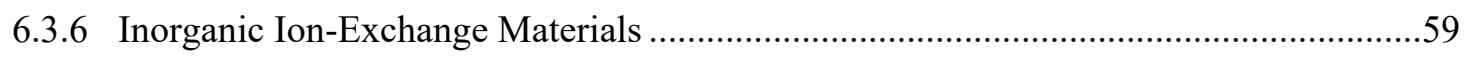

6.3.7 Ion Exchangers on Polymer Supports and Composites...............................................6

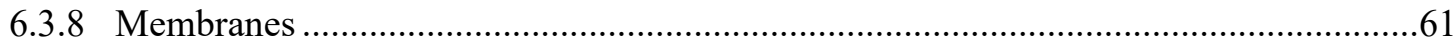

6.3.9 Radiation Effects in Actinide Coordination Chemistry...............................................61

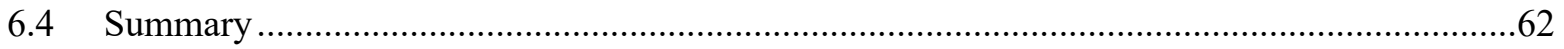

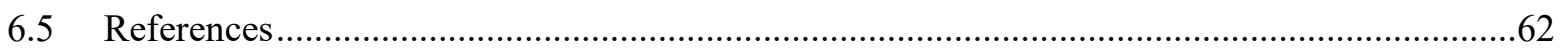

7. FRD 6: Develop Technologies for Real-Time Quantification of Chemical Species .....................66

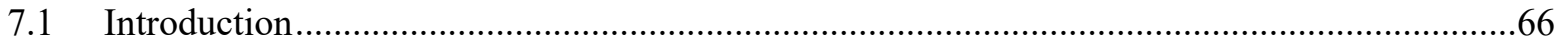

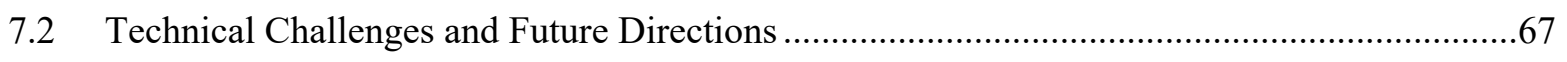

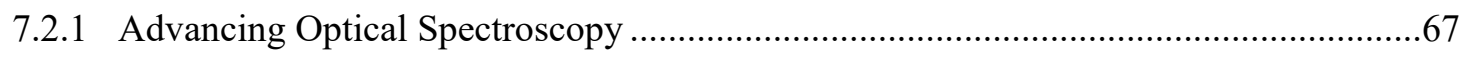

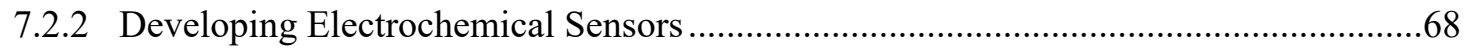

7.2.3 Applying LIBS and Laser Ablation Molecular Isotope Spectrometry (LAMIS)

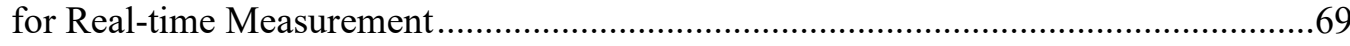

7.2.4 Applying X-ray Spectroscopies to Characterize Nuclear Processing Systems ..............71

7.2.5 Developing Sensitive Sensors for Gas-Phase Species.................................................. 73 
7.2.6 Advancing Volume, Density, and Flow Measurement Technologies...........................74

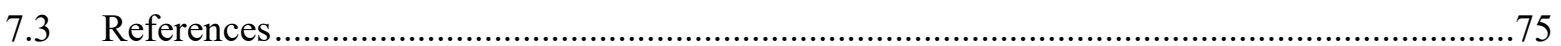

8. FRD 7: Achieve Control of Redox Potential in Molten Salt Systems.............................................79

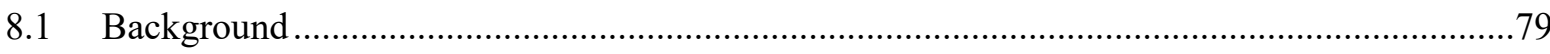

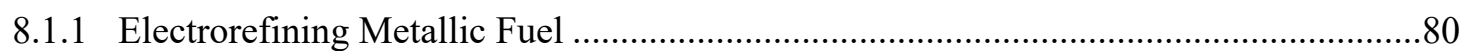

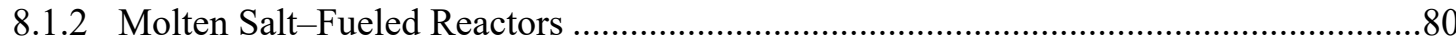

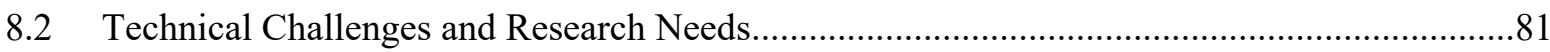

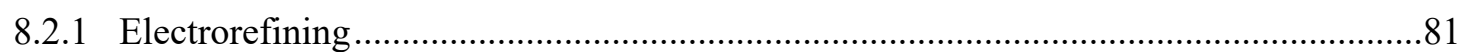

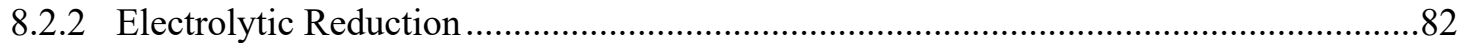

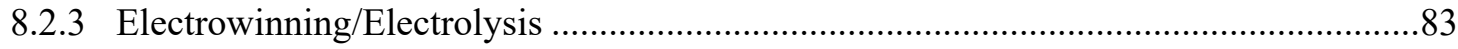

8.2.4 Control of Redox Potential in MSR Fuel Salt ..............................................................84

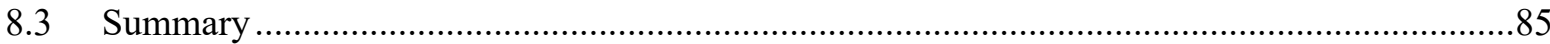

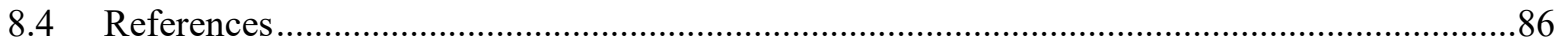

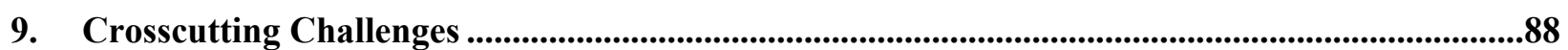

9.1 Accelerate the Rate of Innovation by Leveraging Simulation and Modeling ..........................88

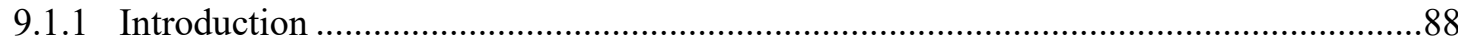

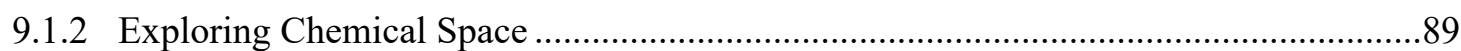

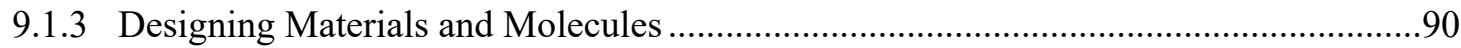

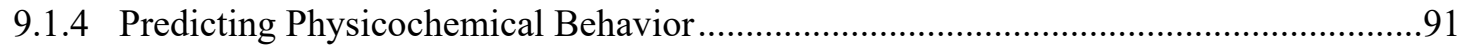

9.1.5 Data Analysis to Reveal the Fundamental Science of Multidimensional

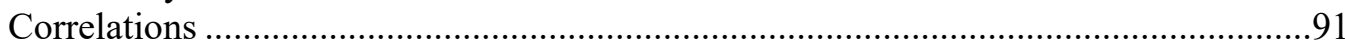

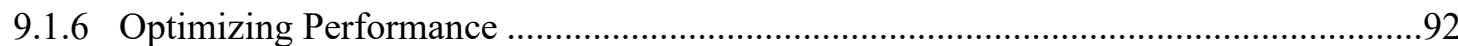

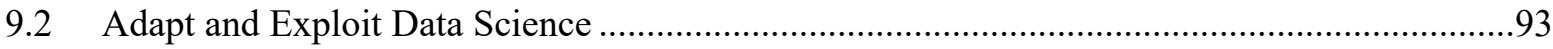

9.2.1 Technical Challenges in Applying Data Science in Advanced Nuclear Fuel

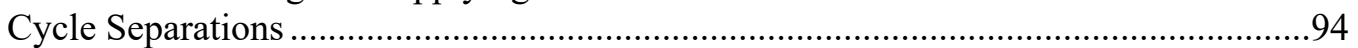

9.2.2 Relevant Data Science Research Needs ..................................................................96

9.3 Understand and Manage Radiation Effects on Materials and Processes ................................97

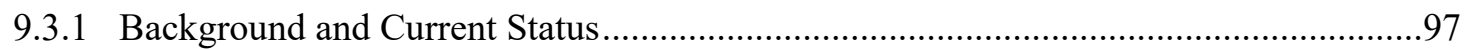

9.3.2 Technical Challenges and Research Directions for Radiation Effects ..........................99

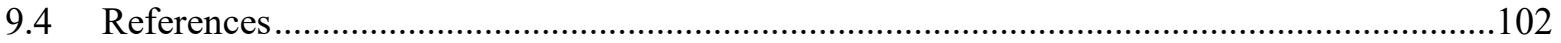

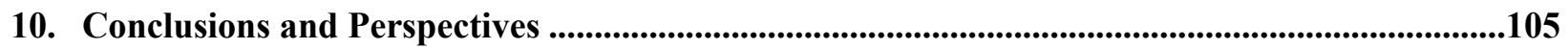

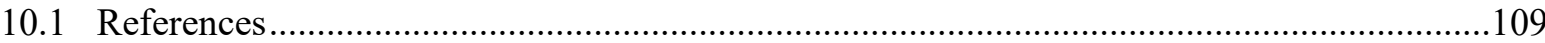

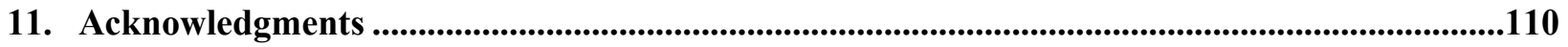

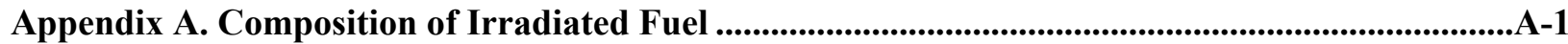

Appendix B. Example Fuel Cycle Options …..............................................................................................1

Appendix C. Current State-of-the-Art: The PUREX Process...................................................................

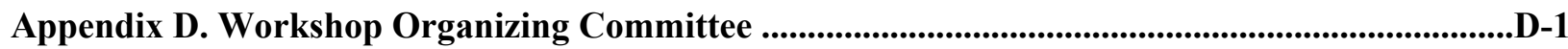

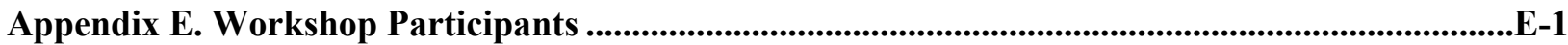

Appendix F. Workshop Agenda ................................................................................................................... 
Appendix G. White Papers ....................................................................................................................................G-1

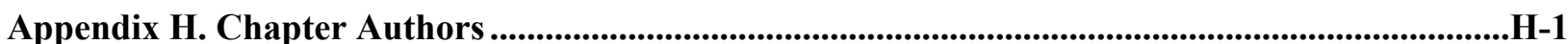




\section{FIGURES}

Figure 1.1. Use of virgin natural $U$ relative to the once-through fuel cycle implementing

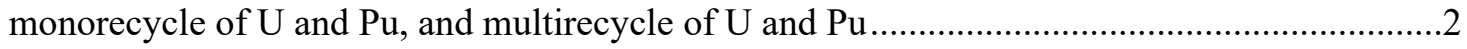

Figure 2.1. Composition of spent nuclear fuel by mass percent of LWRs ................................................

Figure 2.2. Flowchart of chlorination of fuel cladding material of UNF ................................................

Figure 2.3. Flowsheet of $\mathrm{Zr}$ purification process using thionyl chloride ................................................

Figure 2.4. Cross section of a TRISO fuel particle showing the $\mathrm{OPyC}, \mathrm{SiC}$, and IPyC layers and the uranium kernel

Figure 2.5. (a) Diagram of the formation and collapse of a bubble near a liquid/solid interface creating a microjet (Gonzalez-Avila et al. 2020); (b) SEM image of graphite materials and structural damage (pits) after 15 min cavitation erosion time.

Figure 3.1. Conversion of $\mathrm{UO}_{2}$ pellets to $\mathrm{UO}_{3}$ by voloxidation with $\mathrm{NO}_{2}$, and dissolution of the $\mathrm{UO}_{3}$ in TBP solvent.

Figure 3.2. Separation scheme exploiting selective fluorination properties of $\mathrm{NF}_{3}$ . .18

Figure 3.3. Uranium chloride deposits on glass tube after chlorinating and volatilizing uranium metal under flowing $\mathrm{Cl}_{2}$ gas at $500{ }^{\circ} \mathrm{C}$.

Figure 3.4. Schematic representation of a used fuel separation scheme based on carbonateperoxide dissolution of the fuel .23

Figure 4.1. Inspiration from biological molecules in binding and transporting noble gases..... .33

Figure 4.2. Molecular iodine interacts with a sorbent having hierarchical materials design observable at the nanometer, micrometer, and millimeter scale (Matyáš 2021).

Figure 4.3. Light and electric-field induced structural transformation of molecular solids

Figure 5.1. The magnetic susceptibilities of actinide ions suggest the possibility of magnetic separation of ionic species in irradiated fuel.

Figure 6.1. Example of nickel-based cationic MOF material developed for ${ }^{99} \mathrm{TcO}_{4}{ }^{-}$uptake.....................54

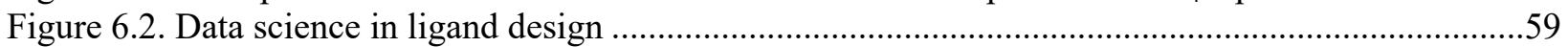

Figure 6.3. Ion exchange of uranyl adsorption by metal sulfide KMS-1 material .................................60

Figure 7.1. Experimental observation of spectra from a variety of process conditions..............................68

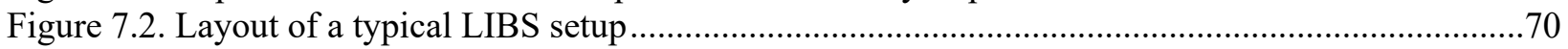

Figure 7.3. Temperature-dependent XANES spectral overlays (one scan per temperature point) of anhydrous $\mathrm{EuCl}_{3}$ and $17.5 \mathrm{~mol} \% \mathrm{EuCl}_{3}-82.5 \mathrm{~mol} \% \mathrm{KCl}$ eutectic composition collected using easyXAFS300 equipped with a miniature vacuum furnace...........................72

Figure 7.4. Design and development of advanced functional materials for sensing application.................74

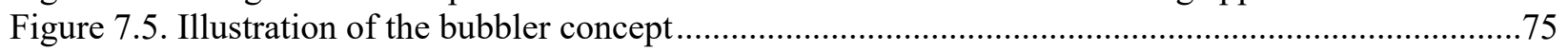

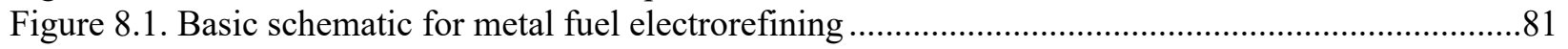

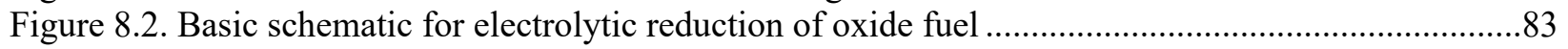

Figure 9.1. A selection of simulation approaches across length scales ...............................................89

Figure 9.2. Schematic for the radiation-induced reduction of nickel cations to nanoparticles in molten zinc chloride

Figure 9.3. Change in the short-wavelength absorption properties of fiber optics with absorbed $\gamma$ dose

Figure 10.1. Future research directions supporting the goal of actinide recycle for sustainable nuclear energy draw from crosscutting research on modeling and simulation, data science, and minimizing radiation effects.....

Figure 10.2. Notable workshop reports dealing with research needs in SS\&T relevant to nuclear fuel cycles

Figure A.1. Postirradiation composition of 1 ton $3.2 \%$ enriched $\mathrm{UO}_{2}$ fuel 10 years after irradiation in a light-water moderated PWR to $33 \mathrm{GWd} / \mathrm{t}$ burnup 
Figure A.2. Postirradiation radioactivity in 1 ton low-enriched uranium dioxide fuel 10 years after irradiation in a light water moderated pressurized water reactor or boiling water reactor

Figure B.1. Example fuel cycle scenario with monorecycle of $\mathrm{U}$ and $\mathrm{Pu}$ B-1

Figure B.2. Example fuel cycle scenario with multirecycle of $\mathrm{U}$ and $\mathrm{Pu}$ B-2

Figure C.1. Chemical structure of TBP C-2

\section{TABLES}

Table 3.1. Relative Volatilities of Fluorides of Significance to Irradiated Fuel Reprocessing ..................18

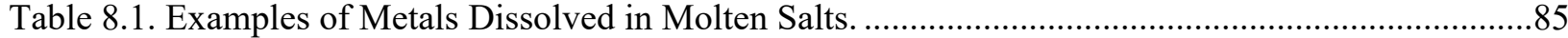

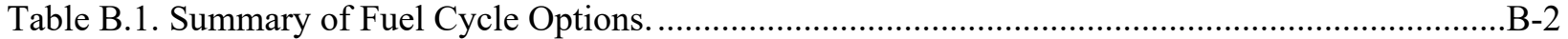




\section{ABBREVIATIONS}

\begin{tabular}{|c|c|}
\hline ADS & accelerator-driven systems \\
\hline AI & artificial intelligence \\
\hline ALSEP & actinide-lanthanide extraction \\
\hline ARPA-E & Advanced Research Projects Agency-Energy \\
\hline BWR & boiling water reactor \\
\hline $\mathrm{COF}$ & covalent organic frameworks \\
\hline DFT & density functional theory \\
\hline DOE & US Department of Energy \\
\hline DOE-NE & US Department of Energy's Office of Nuclear Energy \\
\hline EBR-II & Experimental Breeder Reactor II \\
\hline FP & fission product \\
\hline FRD & future research direction \\
\hline GCMC & grand canonical Monte Carlo \\
\hline HALEU & high-assay low-enriched uranium \\
\hline HDEHP & di(2-ethylhexyl)phosphoric acid \\
\hline HLW & high-level waste \\
\hline INL & Idaho National Laboratory \\
\hline LAMIS & laser ablation molecular isotope spectrometry \\
\hline LEU & low-enriched uranium \\
\hline LIBS & laser-induced breakdown spectroscopy \\
\hline LLW & low-level waste \\
\hline LWR & light water reactor \\
\hline MCFR & molten chloride fast reactor \\
\hline ML & machine learning \\
\hline MOFs & metal-organic frameworks \\
\hline MOX & mixed oxide fuel \\
\hline MSR & molten salt reactor \\
\hline MSRE & Molten Salt Reactor Experiment \\
\hline NEUP & Nuclear Energy University Program \\
\hline ORNL & Oak Ridge National Laboratory \\
\hline PNNL & Pacific Northwest National Laboratory \\
\hline PUREX & plutonium uranium reduction extraction \\
\hline PWR & pressurized water reactor \\
\hline $\mathrm{sc}-\mathrm{CO}_{2}$ & supercritical $\mathrm{CO}_{2}$ \\
\hline SFE & supercritical fluid extraction \\
\hline SNM & special nuclear material \\
\hline SS\&T & separation science and technology \\
\hline TALSPEAK & $\begin{array}{l}\text { trivalent actinide-lanthanide separation by phosphorus reagent extraction from } \\
\text { aqueous komplexes }\end{array}$ \\
\hline TBP & tri- $n$-butyl phosphate \\
\hline THOREX & thorium extraction \\
\hline TRISO & tri-structural isotropic \\
\hline TRL & technology readiness level \\
\hline TRU & transuranium \\
\hline UNF & used nuclear fuel \\
\hline XANES & X-ray absorption near-edge structure \\
\hline XAS & X-ray absorption spectroscopy \\
\hline ZIF & zeolitic imidazole framework \\
\hline
\end{tabular}




\section{EXECUTIVE SUMMARY}

Deployment of advanced nuclear reactors will inevitably introduce new challenges for devising and implementing an efficient, safe, and economical nuclear fuel cycle that meets society's need for clean energy and expectations for environmental stewardship. The growing urgency for decarbonizing the US and global economies makes such technological challenges all the more compelling. The Office of Materials and Chemical Technologies within US Department of Energy's Office of Nuclear Energy stewards the capabilities and knowledge relied upon by government policy makers to make informed decisions regarding nuclear fuel cycle options. Such decisions in turn rely on the development of efficient and economical separation methods that can accept the used nuclear fuel containing actinides and fission products (FPs) to recycle selected actinides, recover valuable by-products, and deliver waste streams that are suitable for disposal.

To help guide the future direction of fuel cycle separations research, taking into account emerging technologies, the Office of Materials and Chemical Technologies sponsored the Innovative Separations R\&D Needs for Advanced Fuel Cycles workshop, held virtually August 30-September 1, 2021. Based upon 60 contributed white papers, 6 plenary lectures, and 3 days of discussions, the outcome of the workshop and subsequent deliberations was the generation of this report identifying seven future research directions (FRDs) plus three crosscutting areas of research. Each FRD is described in an individual chapter in this report as follows:

FRD 1: Develop Methods to Efficiently Remove and Treat Coating and Cladding Materials

FRD 2: Devise Chemistry that Operates Directly on Irradiated Fuel

FRD 3: Design Robust Materials for Separation of Gas-Phase Species

FRD 4: Apply Physical Phenomena, Fields, and Gradients to Intensify Separations

FRD 5: Exploit Principles of Coordination Chemistry to Simplify Actinide Separations

FRD 6: Develop Technologies for Real-Time Quantification of Chemical Species

FRD 7: Achieve Control of Redox Potential in Molten Salt Systems.

The three crosscutting research areas are identified and incorporated as a subsequent chapter:

Crosscut 1: Accelerate the Rate of Innovation by Leveraging Simulation and Modeling

Crosscut 2: Adapt and Exploit Data Science

Crosscut 3: Understand and Manage Radiation Effects on Materials and Processes.

FRD 1. Develop Methods to Efficiently Remove and Treat Coating and Cladding Materials. New technologies are needed to process the advanced fuels, coatings, and claddings integral to advanced fuel cycles, as well as materials from light water reactors. With some exceptions (e.g., molten salt reactors), nuclear fuel cycle separations begin with breaching or removing the cladding or coatings, which can constitute a major fraction of the mass of the used fuel assembly that must be processed. Historically, this has been performed through shearing of the Zircaloy cladding used to contain uranium oxide fuel pellets for light water reactors. Now, concepts such as accident-tolerant fuels, accident-tolerant claddings, and coated particle fuels require new solutions to this first step of separations. Separating these materials for disposal as low-level waste would substantially benefit the utilization of geological repositories intended for high-level waste. Thus, research needs are identified for recovery and decontamination of metallic cladding materials, separation of bulk carbon material from tri-structural isotropic (TRISO)-based fuel assemblies, and exposure of TRISO fuel kernels for subsequent separation activities.

FRD 2. Devise Chemistry that Operates Directly on Irradiated Fuel. This FRD explores methods other than direct dissolution of the fuel in hot nitric acid for accessing the actinide elements in irradiated fuel for recovery and recycle. The approaches seek to mitigate issues that arise from the dissolution, including 
management of nitrate, tritium, and iodine and could result in overall simplification of separations. The methods considered for future research include voloxidation of the fuel followed by direct dissolution in process solvents, halide volatility, supercritical fluid extraction, and alkaline-side processing. Also considered in this FRD are separation methods that act directly on the salt in molten salt reactors. Large swaths of unknown chemistry make this FRD particularly ripe with opportunity.

FRD 3. Design Robust Materials for Separation of Gas-Phase Species. This FRD prompts consideration of how materials design can be used to advance efficient and cost-effective gas-phase separations and monitoring relevant to the nuclear fuel cycle. Volatile elements or chemical compounds can be released by a wide range of fuel cycle operations, spanning fuel irradiation, fuel processing, and waste stabilization. These volatile materials can be released in many forms and may merit attention based on their value (e.g., ${ }^{3} \mathrm{He}, \mathrm{Xe}$ ), their role in the fuel cycle (e.g., $\mathrm{UF}_{6}$ ), or their waste management requirements (e.g., compounds containing $\left.{ }^{129} \mathrm{I},{ }^{3} \mathrm{H}\right)$. Multiple areas in which advanced materials design could be productively applied include sequestration, separations, sensing, or stabilization of gaseous by-products present during recovery of uranium or other valuable materials. High-priority research needs for advancement of gas-solid separations within the nuclear fuel cycle include (1) accelerating materials discovery, (2) advancing structural forms of novel materials, and (3) applying physical stimuli to materials to effect separations. Integration of separations with disposition is an especially important success criterion.

FRD 4. Apply Physical Phenomena, Fields, and Gradients to Intensify Separations. Separations depend on physically isolating species of interest from all other components of a mixture, which requires physical movement of at least some of the components. Consequently, the control and manipulation of properties that can alter the transport or accumulation of specific species in separation systems represent a key approach to alter the efficiency and selectivity of separations. The research priorities outlined in this FRD represent opportunities to design new separations approaches or augment existing separations to achieve critical improvements in the recovery of materials that can intensify separation processes and simplify management of the back end of nuclear fuel cycles. Methods that manipulate separations through the application of external fields, exploit the unique properties of interfaces, and use solubility and novel solvation states constitute fertile research directions.

FRD 5. Exploit Principles of Coordination Chemistry to Simplify Actinide Separations. This FRD highlights the value of coordination chemistry in actinide separations. Many separative strategies are available for actinide recovery, many of which prioritize excellent product purity and yields. Recently, the economic feasibility of actinide recycling has become the main determinant, with process simplification at the forefront of research and development (R\&D) efforts. The design of materials and molecules is integral to finding novel approaches in actinide separations that allow significant process simplification. The discovery efforts must consider six important features of a promising coordination environment as compatibility, selectivity, affinity, kinetics, stability, and predictability are all important physicochemical properties that contribute to process development. The identified challenges are to (1) improve the understanding of $f$-element selectivity, (2) accelerate ligand design, (3) manage fission products, (4) predict reagent stability, which all must deliver (5) overall process simplification. A well-coordinated link between the experiment and theory can further advance the theoretical description of actinide binding, benefiting from a data-science approach. Machine learning tools, theoretical modeling, and high-throughput synthesis and screening will open new approaches to intelligent ligand design and chemical separations, sampling of vast areas of chemical space. Research needs are recognized for understanding and exploiting the coordination chemistries of actinides and ruthenium, zirconium, niobium, iodine, and technetium in nuclear fuel cycles, as management of those particular fission products is most problematic to actinide separations. Novel actinide coordination environments will inherently sustain chemical and radiolytic degradation. Understanding of such degradation processes must be mastered at the molecular level, developing recommendations on reagent classes and strategies to build in robustness. Such R\&D efforts may also be 
uniquely transformed with a data-science approach, computations, and high-throughput synthesis and screening.

FRD 6. Develop Technologies for Real-Time Quantification of Chemical Species. Economics and safeguarding of special nuclear materials (SNM) are two significant barriers. This FRD describes methods to enable real-time or near real-time quantification of critical constituents in nuclear recycling streams. Multiple techniques are desired for an advanced plant, as each generally has limitations and provides unique information. Combinations of techniques provide enhanced reliability, utility, and safeguarding of SNM. Analytical methods that would benefit from research include optical spectroscopy (absorbance and Raman), electrochemical sensors, laser-induced breakdown spectroscopy, laser ablation molecular isotope spectrometry, X-ray spectroscopy techniques, and sensors for gas-phase species. Advancements in instrumentation and data analysis techniques (e.g., chemometric modeling) will push these technologies forward to enable more rapid process development and eventual deployment in processing plants for the purposes of process monitoring and control, as well as safeguarding of special nuclear materials. Research needs for the related monitoring requirements for solution volumes, densities, and flowrates are also identified.

FRD 7. Achieve Control of Redox Potential in Molten Salt Systems. This FRD focuses on separations from molten salts, which are important for processing metallic fuel or molten salt fuel irradiated in advanced reactors. Molten salts are generally accepted to be highly if not entirely ionic in structure, and thus electrochemistry is a key approach to achieving selective separations. Separation can be driven by changing actinides from salt-soluble to salt-insoluble oxidation states. Research needs for pyroprocessing include selectivity for uranium and transuranic elements in recovery operations, anode stability, reference-electrode stability, and improving processing rate. Molten salt fuel processing is relatively underdeveloped. Leveraging the science and technology from metallic fuel pyroprocessing with modifications and optimization is a ripe area for future research.

Crosscut 1. Accelerate the Rate of Innovation by Leveraging Simulation and Modeling. Modeling and simulation offer the potential to "turbocharge" the rate of research aimed at new separation technologies relevant to nuclear fuel cycles. Computational techniques have permeated and already accelerate modernday scientific research at the project level, yet a more holistic vision encompasses the incorporation of modeling and simulation throughout a full cycle of R\&D leading to functional separation systems. This R\&D workflow consists of an explore-design-predict-analyze-optimize paradigm in which modeling and simulation play a central role exploring the free energy of separation processes, designing new materials and molecules tailored for meeting the goals in the FRDs, predicting physicochemical behavior in separation systems, analyzing the multiscale functional attributes of separation systems, and finally optimizing the functional performance to realize an efficient separation.

Crosscut 2. Adapt and Exploit Data Science. Data science is a broadly applicable and growing interdisciplinary field that incorporates statistical methods, data analysis, informatics, and related algorithms. This field presents a major opportunity for making quantum leaps in technology needs identified in multiple FRDs, including real-time monitoring and design of separation agents with superior properties. Use of data science has so far been scant in research for nuclear fuel cycle separations. The major barriers lie in developing the mega databases needed as well as the algorithms that will access the data and use it to predict new materials and processes. Critical capabilities needed include building reliable and accessible databases, adapting generic machine learning models for chemistry, and developing high-throughput experimental techniques.

Crosscut 3. Understand and Manage Radiation Effects on Materials and Processes. Radiation effects are ubiquitous throughout all aspects of managing used nuclear fuel and underpin the feasibility of a given separations technology. Radiation-induced processes promote the destruction of molecules and materials 
with the formation of potentially detrimental degradation products and corresponding changes in physical and chemical properties, thereby impacting the effectiveness and longevity of a separations process. Although destructive effects are usually associated with radiation, beneficial effects can be foreseen. To support the accelerated development of separation technologies for advanced fuel cycles and fuels, a molecular-level understanding of radiation effects must therefore be attained over multiple time, distance, and material domains. This requires a transition from empirical measurement to accurate computer-based predictive capabilities for benchtop to plant scale by addressing the following technical challenges: (1) identifying and manipulating radiation-induced synergies for the efficient removal and treatment of coating and cladding materials in advanced reactor fuel forms; (2) evaluating the radiation robustness of materials designed for the separation of gas-phase species; (3) understanding and mastering the effects of radiation on complexants intended to simplify and compact actinide separations; (4) correlating radiationinduced changes in physical and chemical properties with the response of real-time quantification of chemical species in nuclear processing streams; and (5) integrating multiscale radiation modeling methods with validated computer models and data science approaches for accelerating the rate of reprocessing innovation.

The emerging technologies and future research directions identified here along with three crosscutting research topics aim to solve separation problems that will enable safe, secure, economical, and environmentally responsible recycling of actinides needed for advanced fuel cycle options. As the demand for economical clean energy grows more acute toward domestic and global decarbonization targets, pursuit of the research goals described in this report will more precisely inform future decision-makers in choices among the available fuel cycles. Separation science and technology (SS\&T) is foundational to the nuclear enterprise. Since the dawn of the nuclear era in the late 19th and early 20th centuries, SS\&T has enabled the advancement of neutron science and technology, leading to commercial nuclear power on a global scale. To gain full advantage of emerging nuclear power reactor technology, the continued development of nuclear SS\&T is essential. The physics underlying a sustainable nuclear power future - multirecycle of actinides into a fleet of thermal and fast-spectrum power reactors - is well understood. Advancements in the FRDs supported by crosscutting research identified in this report will underpin the implementation of this vision. 


\section{Introduction}

To avoid human-induced catastrophic climate change, the international community is driving toward a netzero carbon economy. The production of electrical power through the application of nuclear fission will play a central role in achieving the net-zero vision (IAEA 2021). The role of nuclear power in reducing carbon emission is recognized in the strategic plan of the US Department of Energy's Office of Nuclear Energy (DOE-NE), which was issued in January 2021 (DOE-NE 2021) and in other initiatives being pursued by the US government. ${ }^{1}$ This effort will necessitate an expansion in the number of nuclear power stations deployed, with new power plants employing new reactor technologies. To support this trend, DOE$\mathrm{NE}$ has instituted programs to accelerate the development and deployment of advanced reactor technologies. ${ }^{2}$ Deployment of these advanced reactors will inevitably introduce new challenges with respect to the nuclear fuel cycle, particularly the management and disposition of irradiated fuels generated in these reactors. ${ }^{3}$ The Office of Materials and Chemical Technologies within DOE-NE stewards the capabilities and knowledge relied upon by government policy makers to make informed decisions regarding nuclear fuel cycle options. Such decisions in turn rely on the development of efficient and economical separation methods that can accept the used nuclear fuel (UNF) containing actinides and fission products (FPs) to recycle selected actinides and deliver suitable streams for disposal.

In 2011 DOE-NE chartered a study to evaluate and screen nuclear fuel cycle options (Wigeland, Taiwo et al. 2014). ${ }^{4}$ The study specified nine evaluation criteria to identify promising fuel cycle options compared with the current once-through nuclear fuel cycle in the United States. These criteria included proliferation risk, nuclear material security risk, safety, environmental impact, resource utilization, development and deployment risk, institutional issues, and financial risk and economics. At the end of this process, 40 groups of fuel cycles were evaluated and were found sufficient to comprehensively represent all fuel cycle options to inform on their potential for providing substantial improvements. The study found four most promising fuel cycles, all involve recycling $\mathrm{U}, \mathrm{Pu}$, and transuranic elements (TRU):

1. Continuous recycle of $\mathrm{U} / \mathrm{Pu}$ with new natural $\mathrm{U}$ fuel in fast critical reactors

2. Continuous recycle of U/TRU with new natural $U$ fuel in fast critical reactors

3. Continuous recycle of U/TRU with new natural $U$ fuel in both fast and thermal critical reactors

4. Continuous recycle of $\mathrm{U} / \mathrm{Pu}$ with new natural $\mathrm{U}$ fuel in both fast and thermal critical reactors.

The operation of each reactor type was further divided according to variation in fissile type: $\mathrm{Pu} / \mathrm{U},{ }^{233} \mathrm{U} / \mathrm{Th}$, and TRU/U. Eight of the 21 continuous recycle concepts involved the use of the ${ }^{233} \mathrm{U} / \mathrm{Th}$ breeder concept. In terms of solid fueled reactors, continuous fuel recycling machines and burner reactors included heavy water reactors, pressurized water reactors (PWRs), sodium fast reactors, and accelerator-driven systems. The molten salt reactor (MSR) concepts included the ${ }^{233} \mathrm{U} / \mathrm{Th}$ breeder concept, molten salt fast reactor concepts, and the accelerator-driven MSR systems. Because the DOE-NE study was chartered in 2011, the current revival of MSR concepts was underrepresented.

Following the release of the DOE-NE fuel cycle options report, a review of the impact of various nuclear fuel cycles on nuclear waste management metrics from each of the 40 fuel cycles discussed in the study above was published (Stauff, Kim et al. 2015). The review provides calculations of the mass, activity, toxicity, and decay heat of spent nuclear fuel and high-level waste at 10,100, and 100,000 years after

\footnotetext{
${ }^{1}$ For example, https://www.state.gov/program-to-create-pathways-to-safe-and-secure-nuclear-energy-included-inbiden-harris-administrations-bold-plans-to-address-the-climate-crisis/.

2 See https://www.energy.gov/ne/advanced-reactor-demonstration-program.

${ }^{3}$ See Appendix A for a generalized discussion of the composition of irradiated nuclear fuel.

${ }^{4}$ See Appendix B for a discussion of possible fuel cycle options.
} 
reactor discharge. The overarching takeaway from this study was that regardless of reactor or fuel type, the long-term wastes generated by reactors that are coupled with continuous fuel recycle strategies (solid fuels) or periodic cleaning (MSR fuels) are significantly lower than those that are not. Furthermore, studies by the Organisation for Economic Co-Operation and Development Nuclear Energy Agency (NEA) have demonstrated the overwhelming positive effects of multirecycling of $\mathrm{Pu}$ on the front end of the nuclear fuel cycle with a greater than $99 \%$ reduction in the amount of virgin $U$ needing to be mined (Figure 1.1) (NEA 2006; NEA 2021). Given that both uranium mining and high-level waste disposal encounter public scrutiny and opposition at the front and back end of nuclear fuel cycle, the societal benefits of fuel recycle strategies and resultant increased likelihood of public acceptance of nuclear energy are clear.

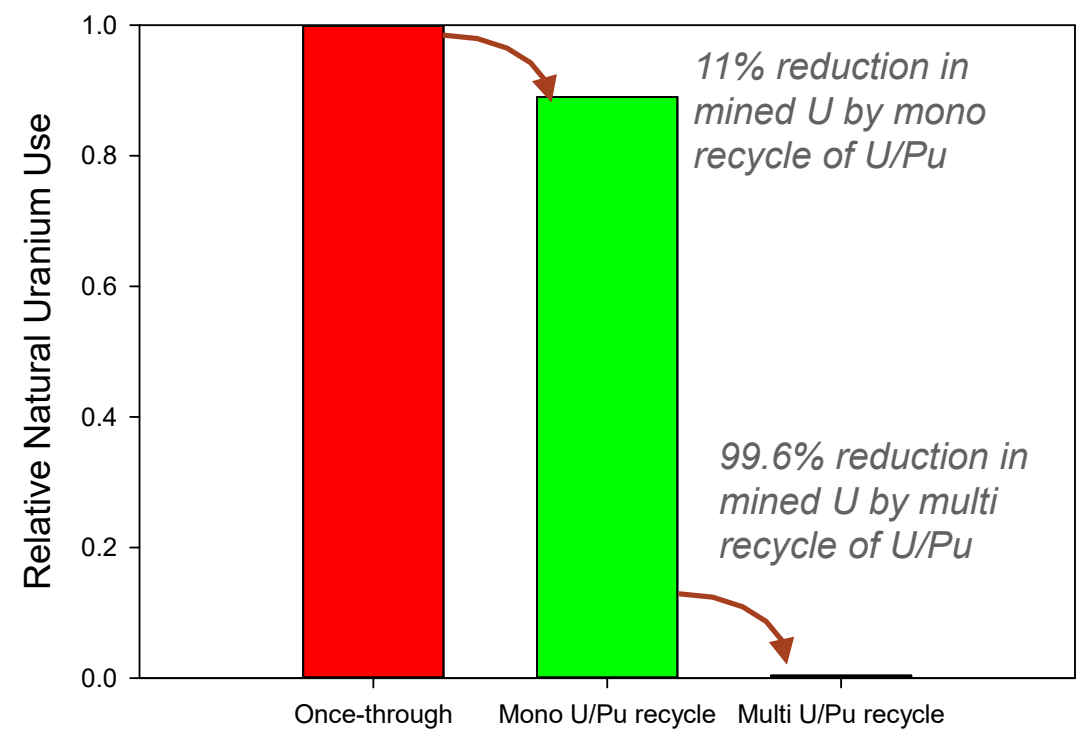

Figure 1.1. Use of virgin natural $U$ relative to the once-through fuel cycle $(r e d)$ implementing monorecycle of $U$ and $\mathrm{Pu}$ (green), and multirecycle of $\mathrm{U}$ and $\mathrm{Pu}$ (black). (Courtesy of PNNL. Based on data from NEA 2006.)

These prior studies point to the need for DOE-NE Office of Materials and Chemical Technologies to develop technical options for recycling actinide materials from advanced power reactors for the United States to be positioned to respond to evolving policy choices within the many existing constraints (economics, proliferation resistance, environmental impact, public acceptance, etc.). The problem is particularly challenging not only because of the uncertainty in future policy choices bearing upon what will be recycled and how to dispose of the residuals but also because of the wide variety of fuel types that might be deployed, such as tri-structural isotropic (TRISO), metallic, or molten salt fuels. Thus, because the parameters of the applicable separation problem currently remain undefined, research by necessity must adopt an options-based or "toolbox" approach. To provide a point of reference for such an approach, a highlevel description of the plutonium uranium reduction extraction (PUREX) process is provided in Appendix C. The PUREX process represents the current state-of-the-art technology for a single recycle of $\mathrm{U}$ and $\mathrm{Pu}$ from irradiated commercial nuclear reactor fuel at plant scale. Although PUREX is highly mature, other fuel cycle options have been less developed to varying degrees. Examples are given in Appendix B. Progress in developing new separations chemistry relevant to many of these options has been reviewed (NEA 2018). To help guide the future direction of fuel cycle separations research, taking into account such emerging technologies, DOE-NE Office of Materials and Chemical Technologies sponsored the Innovative Separations R\&D Needs for Advanced Fuel Cycles workshop, which was held virtually August 30September 1, 2021. 
The workshop spanned three days, including six plenary presentations and five breakout sessions. The purpose of the workshop was to identify opportunities for improving nuclear fuel cycle separations and to provide the basis for corresponding future research directions (FRDs) for the DOE-NE Office of Materials and Chemical Technologies. The workshop generated enthusiastic discussion about new separation concepts with corresponding approaches and methods, producing a list of proposed research needs. The workshop organizing committee consisted of representatives from laboratories across the US Department of Energy (DOE) complex as well as academia (Appendix D). A total of 103 participants registered for the workshop (Appendix E) and 60 white papers with relevant research ideas were submitted in advance of the workshop. Representation was diverse, including 53 participants from DOE national laboratories, 30 from academia, 7 from industry, 8 from DOE, and 5 foreign participants. Nine national laboratories were represented.

Six plenary lectures (shown in the agenda in Appendix F) served to provide a foundation for discussion of the white papers. The topics included a DOE program overview, description of major fuel cycle options, research needs encountered in process implementation for both aqueous and pyrochemical systems, and separation requirements for waste disposition. A summary of the Advanced Research Projects AgencyEnergy (ARPA-E) CURIE (Cleaning Up Radioisotope Enventories) workshop that had been held approximately 1 month earlier provided useful background about improving the economics of reprocessing.

Each nominally only one page in length, the white papers constituted the primary input for concepts discussed during the workshop, ultimately informing the content of this report. The complete list of white papers is attached in Appendix G. Three simultaneous breakout sessions divided the white papers into categories, roughly according to whether the separation concept primarily dealt with a solid-gas, solidliquid, or liquid-liquid system. Albeit with some overlap in topics, this categorization served to group participants according to common interests in different parts of used fuel processing. The breakout sessions on the first day of the workshop allowed each white paper author to present their concept with minimal panel discussion. On the second day, the breakout sessions reconvened to discuss the white papers in more depth.

The third day was devoted to taking the output from the first two days and producing a list of research needs. By the conclusion of the workshop, the organizing committee had gathered a tremendous amount of information along with a list of approximately 20 research needs. In the ensuing weeks, the list was assimilated and distilled to a concise list of FRDs and crosscutting research areas.

The outcome of the workshop and subsequent deliberations was the generation of this report with identification of seven FRDs plus three crosscutting areas of research. Each FRD is described in an individual chapter in this report. The seven FRDs are roughly organized according to the process flow in a fuel reprocessing scheme:

FRD 1: Develop Methods to Efficiently Remove and Treat Coating and Cladding Materials

FRD 2: Devise Chemistry that Operates Directly on Irradiated Fuel

FRD 3: Design Robust Materials for Separation of Gas-Phase Species

FRD 4: Apply Physical Phenomena, Fields, and Gradients to Intensify Separations

FRD 5: Exploit Principles of Coordination Chemistry to Simplify Actinide Separations

FRD 6: Develop Technologies for Real-Time Quantification of Chemical Species

FRD 7: Achieve Control of Redox Potential in Molten Salt Systems

The three crosscutting research areas were identified and incorporated as a subsequent chapter as follows: 
- Accelerate the Rate of Innovation by Leveraging Simulation and Modeling

- Adapt and Exploit Data Science

- Understand and Manage Radiation Effects on Materials and Processes.

Overall, the resulting report is intended to inform DOE-NE decision-making in developing its research strategy for separations supporting sustainable nuclear fuel cycles.

\subsection{References}

DOE-NE. Office of Nuclear Energy: Strategic Vision. 2021, US Department of Energy, https://www.energy.gov/sites/default/files/2021/01/f82/DOE-NE\%20Strategic\%20Vision\%20Web\%20-\%2001.08.2021.pdf.

IAEA. Nuclear Energy for a Net Zero World. 2021, International Atomic Energy Agency, https://www.iaea.org/sites/default/files/21/10/nuclear-energy-for-a-net-zero-world.pdf.

NEA. Advanced Nuclear Fuel Cycles and Radioactive Waste Management. 2006, Nuclear Energy Agency, Organisation for Economic Co-Operation and Development, Paris, France.

NEA. State-of-the-Art Report on the Progress of Nuclear Fuel Cycle Chemistry. 2018, Nuclear Energy Agency, Organisation for Economic Co-Operation and Development, Paris, France.

NEA. Strategies and Considerations for the Back End of the Fuel Cycle. 2021, Nuclear Energy Agency, Organisation for Economic Co-Operation and Development, Paris, France.

Stauff, N. E.; Kim, T. K.; Taiwo, T. A. Variations in Nuclear Waste Management Performance of Various Fuel-Cycle Options. Journal of Nuclear Science and Technology 2015, 52, 1058-1073.

Wigeland, R.; Taiwo, T.; Ludewig, H.; Todosow, M.; Halsey, W.; Gehin, J.; Jubin, R.; Buelt, J.; Stockinger, S.; Jenni, K.; Oakley, B. Nuclear Fuel Cycle Evaluation and Screening-Final Report. INL/EXT-14-31465. Idaho National Laboratory, 2014. 


\section{FRD 1: Develop Methods to Efficiently Remove and Treat Coating and Cladding Materials}

\begin{abstract}
New technologies are needed to process the advanced fuels, coatings, and claddings integral to advanced fuel cycles, as well as materials from light water reactors (LWRs). With some exceptions (e.g., MSRs), nuclear fuel cycle separations begin with breaching or removing the cladding or coatings, which can constitute a major fraction of the mass of the used fuel assembly that must be processed. Historically, this has been performed through shearing of the Zircaloy cladding used to contain uranium oxide fuel pellets for LWRs. Now, concepts such as accident-tolerant fuels, accident-tolerant claddings, and coated particle fuels require new solutions to this first step of separations. Separating these materials for disposal as low-level waste (LLW) would substantially benefit the utilization of geological repositories for high-level waste. Thus, research needs are identified for recovery and decontamination of metallic cladding materials, separation of bulk carbon material from TRISO-based fuel assemblies, and exposure of TRISO fuel kernels for subsequent separation activities.
\end{abstract}

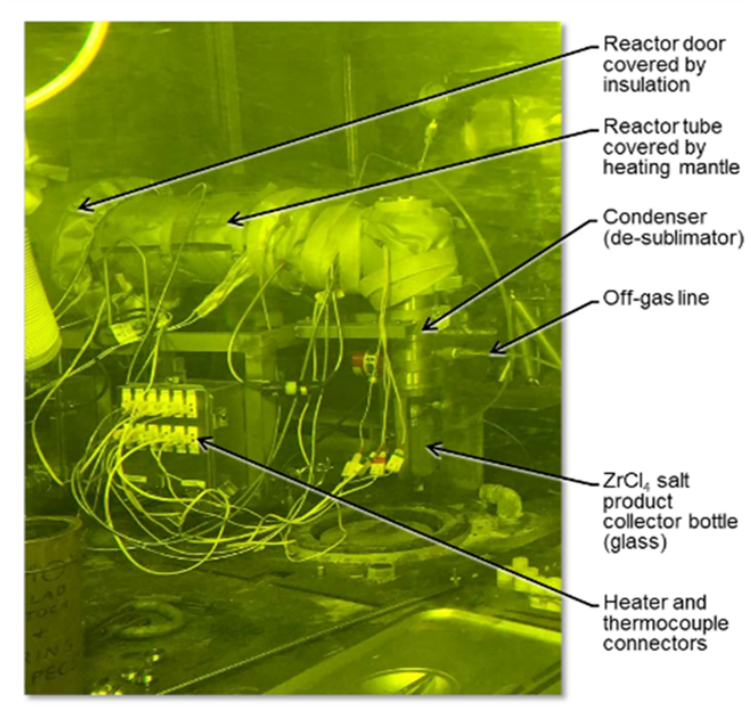

Chlorination of irradiated Zircaloy cladding segments in the Oak Ridge National Laboratory (ORNL) Hot Fuel Examination Facility. (Photo courtesy of ORNL.)

\subsection{Introduction}

The urgent need to decarbonize the energy sector is currently being addressed in the United States and worldwide. Interest in nuclear power production has increased because of its minimal generation of greenhouse gases and its ability to provide steady baseload power regardless of weather conditions. Developing and transitioning toward new generations of reactor technologies requires a strong emphasis on solution-oriented research that provides recycling and disposal strategies for both the used advanced reactor fuel and its coating or cladding material.

Many advanced nuclear reactor designs are based on using high-assay low-enriched uranium (HALEU) fuel, which has ${ }^{235} \mathrm{U}$ enrichments up to $19.5 \%$ and greatly exceeds the common $3-5 \%{ }^{235} \mathrm{U}$ enrichment levels used in Generation III reactors. Thus, depending on the burnup achieved, used HALEU fuel could still have $U$ enrichment above natural levels, increasing the economic attractiveness of $U$ recycling for HALEU fuels. As advanced reactor designs are demonstrated and deployed commercially, forecasts for HALEU supply requirements show a rapid increase with significant continuing demand. This increasing need for HALEU is a primary driver for reprocessing advanced fuels. Some of the coating and cladding materials of advanced fuels are challenging to penetrate and thus require the development of new strategies for exposing the used fuel for subsequent processing and dealing with residual coatings and claddings.

A second driver for developing new cladding and coating removal technologies is the ability to dramatically reduce the high-level waste (HLW) associated with UNF processing. Current international practice at commercial fuel reprocessing facilities of LWR fuel entails decladding of Zr-based cladding hulls via fuel segmentation through a mechanical shearing process, followed by acid dissolution of the fuel matrix out of the cladding. The empty cladding hulls contain embedded radionuclides that cannot be removed by acid 
washing. Therefore, they require final disposal as HLW, consuming valuable space in a deep geological repository.

In case of direct disposal of intact UNF, this strategy will include the cladding and matrix material as well as the fissile material (often $U$ ). If the claddings and coatings are disposed of as part of an intact fuel assembly, they represent a large amount of the waste, with $25 \%$ of the mass requiring disposition as HLW for LWR fuel. Alternatively, cladding and coating materials can be removed and decontaminated from the fissile material and FPs, allowing for their disposal as intermediate or low-level waste (LLW).

To maximize the fuel cycle improvements promised by the use of HALEU fuels and to minimize the amount of HLW produced, the following research needs have been identified to develop new cladding and coating management strategies:

1. Removing and decontaminating current and future cladding materials

2. Removing coating materials from TRISO-based fuels for accessibility to recycle valuable elements

\subsection{Research Needs for Decladding and Decontamination}

UNF reprocessing technology industrially applied to LWR fuels is currently based on the PUREX solvent extraction process (Appendix C), which involves the associated steps of fuel disassembly and dissolution, conversion of uranium and plutonium to oxide products, and vitrification of the minor actinide-FP fraction into a robust borosilicate waste form. Compaction of the cladding into a metallic waste form has been deployed in France as a means of waste volume reduction.

However, the separated cladding still classifies as intermediate-level waste in France and would classify as HLW in the United States because it still contains embedded radionuclides. Thus, this irradiated cladding material requires disposal by emplacement in a geologic repository in the United States in comparable volumes of vitrified high-level waste and technological wastes. Below, emerging technologies and future research needs are identified for waste minimization and recycling of valuable elements via decladding approaches.

Zircaloy cladding is the second largest mass (about 25\%) of all components in used US LWR fuels (Figure 2.1), based on the US inventory of used fuel through July 2002 (Collins et al. 2010). The inventory consists of $71 \%$ PWR used fuel and $29 \%$ boiling water reactors (BWR) used fuels, calculated on the basis of a 40 year decay period. Currently, used fuel is generated at a rate of about 2,000 MT/year. With expected growth over the next 40 years, the generation rate could increase to about 3,000 MT/year, containing about 1,000 MT of Zr. Owing to the large amount of cladding material, there is great incentive to explore other more economical disposal strategies than the direct disposal of spent fuel or the disposal of cladding material as HLW after the decladding process currently applied in nuclear fuel recycling.

Because of the embedded radionuclides that cannot be removed by acid washing, research and development (R\&D) was undertaken in 2010-2018 to recover and purify the $\mathrm{Zr}$ (98\% of the Zircaloy cladding) to enable reuse or disposal as LLW (Collins, et al. 2017). The major challenge was to remove tritium and other embedded radioactive elements such as U, TRUs, FPs, and activation products. Previous R\&D on $\mathrm{Zr}$ recycling explored dry pyrochemical chlorination methods using $\mathrm{Cl}_{2}$ or $\mathrm{HCl}$ and decontamination and purification of the Zircaloy in older fuel claddings, which contained primarily $\mathrm{Sn}$ as the alloying agent. A flowchart of the process is depicted in Figure 2.2. The chlorination method is a two-stage process. In a first step, the cladding material is heated and exposed to chlorine gas $\left(\mathrm{Cl}_{2}\right)$ at temperatures at $350-380{ }^{\circ} \mathrm{C}$ to form and sublime volatile $\mathrm{ZrCl}_{4}$. Most of the impurities are either more volatile or less volatile. A secondary 
purification via chlorination is sufficient to purify the Zr from Zircaloy-2 or Zircaloy-4 (the latter is still used in BWR cladding).

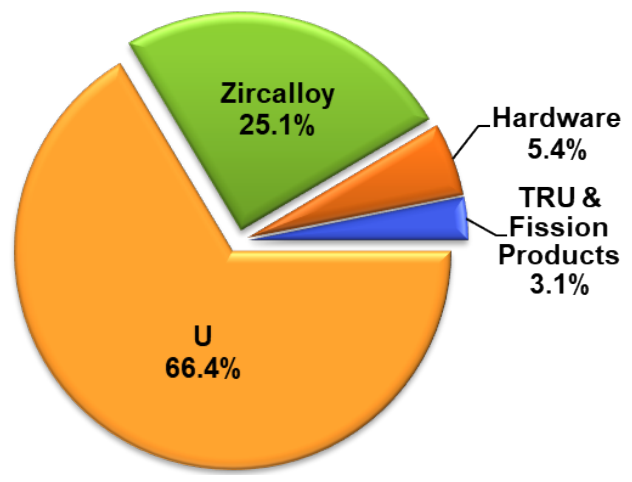

Figure 2.1. Composition of spent nuclear fuel by mass percent of LWRs (Collins et al. 2010).

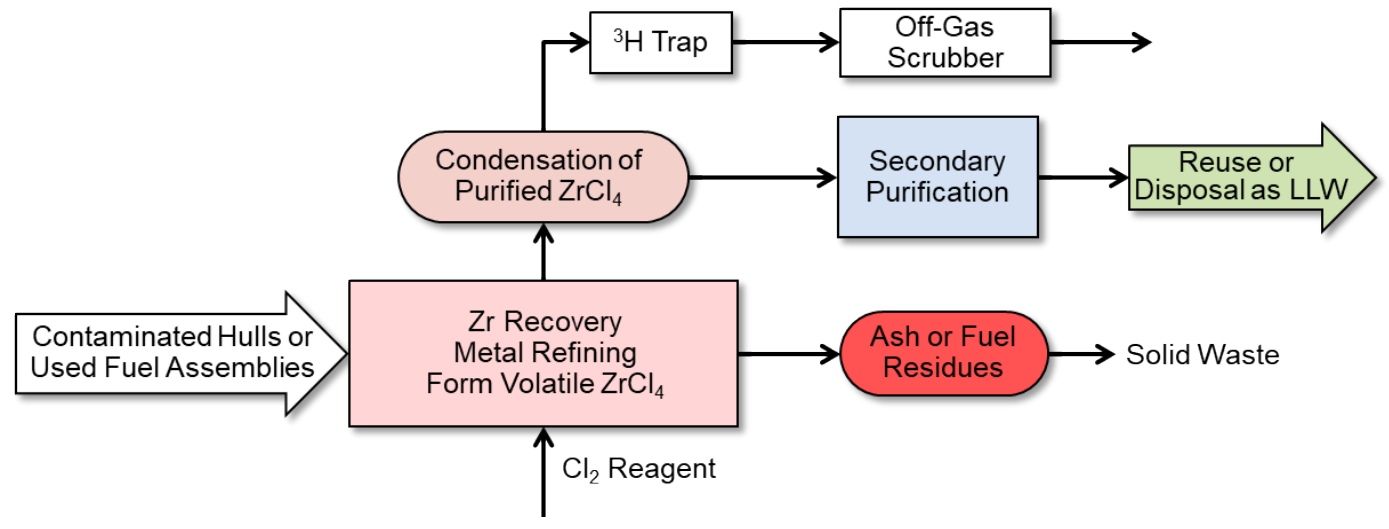

Figure 2.2. Flowchart of chlorination of fuel cladding material of UNF. (Courtesy of ORNL.)

However, since about $1990, \mathrm{Zr}$ cladding containing $1 \% \mathrm{Nb}$ as a major alloying agent has been used for PWR fuel, complicating separations. During reactor operation and irradiation, $\mathrm{Nb}$ is activated to ${ }^{94} \mathrm{Nb}$ and is converted to ${ }^{94} \mathrm{NbOCl}_{3}$ during chlorination, which has a similar vapor pressure as $\mathrm{ZrCl}_{4}$, making the above-described approaches no longer suitable for removal of $\mathrm{Zr}$ for recycling purposes. The ${ }^{94} \mathrm{Nb}$ has a 20,000 year half-life and a strong $\gamma$ emission. Therefore, a more extensive and shielded secondary purification of $\mathrm{Zr}$ is required. A Nuclear Energy University Program (NEUP) study was initiated in 2016 at the University of Nevada Las Vegas and the University of Tennessee, Knoxville, to develop a purification process to remove $\mathrm{Nb}$ from $\mathrm{Zr}$. During these efforts, the University of Tennessee process used a stronger chlorinating agent than $\mathrm{Cl}_{2}$, thionyl chloride $\left(\mathrm{SOCl}_{2}\right)$, to convert $\mathrm{NbOCl}_{3}$ to $\mathrm{NbCl}_{5}$, which is more volatile than $\mathrm{ZrCl}_{4}$ and thus can enable a partial separation of $\mathrm{Nb}$ from $\mathrm{Zr}$. However, if the conversion of the cladding material into LLW is the goal, simpler and more effective purification using aqueous or electrochemical processing may be possible and should be explored.

Advancement of methods for $\mathrm{Zr}$ purification and recycle are needed. Continued NEUP research at the University of Tennessee, Knoxville, has developed an alternative $\mathrm{Zr}$ recovery and purification process that uses dissolution of $\mathrm{ZrCl}_{4}$ and subsequent recrystallization in liquid $\mathrm{SOCl}_{2}$ (Barnes et al. 2021). A diagram of the process is shown in Figure 2.3. Chlorination and dissolution of several types of $\mathrm{Zr}$ alloy cladding is slow (days) in refluxing $\mathrm{SOCl}_{2}\left(\mathrm{bp} \sim 80{ }^{\circ} \mathrm{C}\right.$ ) but accelerated significantly during pressurized refluxing at higher temperatures, thereby achieving practical rates. When the final solution is cooled to room 
temperature, the $\mathrm{ZrCl}_{4} \cdot \mathrm{SOCl}_{2}$ adduct is obtained as a crystalline solid. After filtration or centrifugation of the mother liquor, purified $\mathrm{ZrCl}_{4}$ can be obtained by mild heating under vacuum or in a stream of dry nitrogen, and the released $\mathrm{SOCl}_{2}$ can be recycled. Future research could benefit from demonstrations using irradiated fuel, application to other cladding types, and equipment development and scale up. In the longterm, alternatives to chlorination may be worth considering as approaches to reduction of the mass of cladding material destined for disposal.

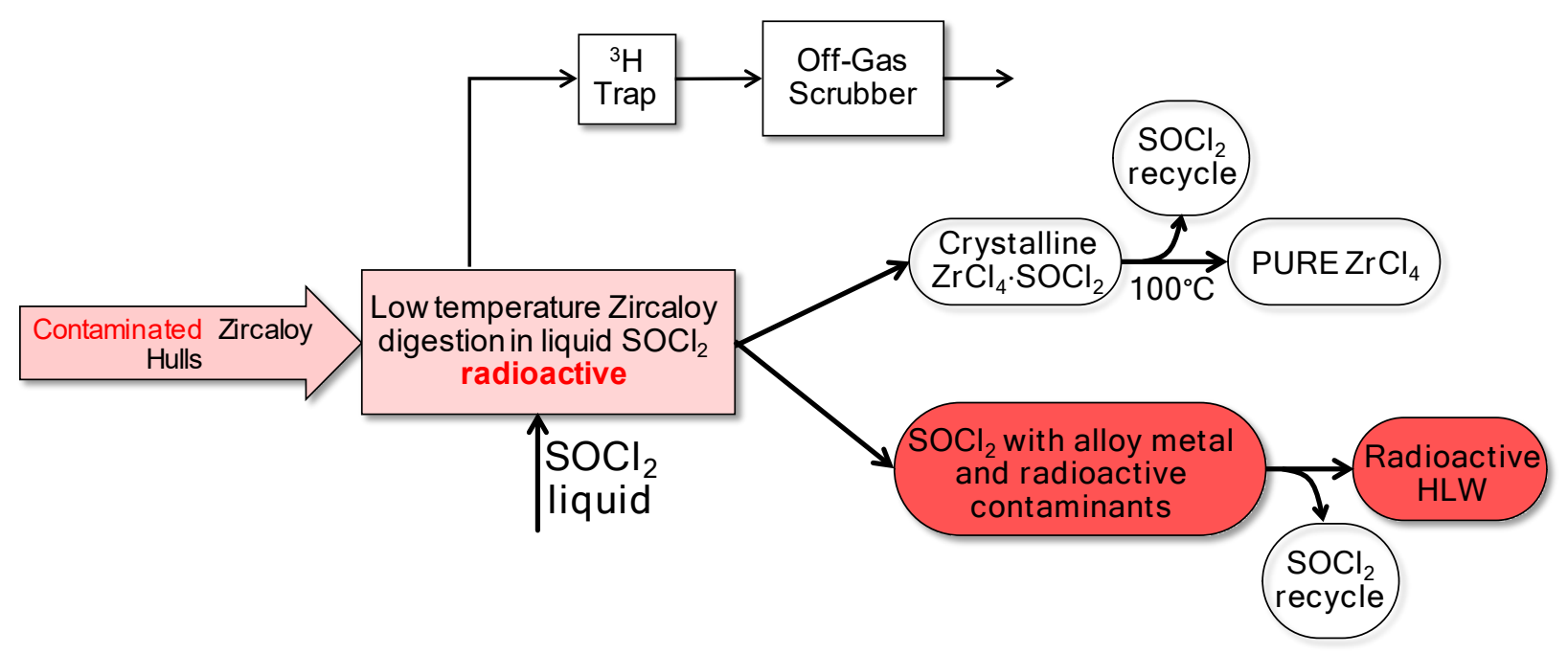

Figure 2.3. Flowsheet of $\mathrm{Zr}$ purification process using thionyl chloride $\left(\mathrm{SOCl}_{2}\right)$. (Courtesy of ORNL.)

Options for reprocessing and component recycle of claddings from accident-tolerant fuels are needed. In the near future, accident-tolerant fuel claddings may be widely deployed in existing and new power reactor fuels. These claddings include chromium-coated zirconium alloy and iron-chromium-aluminum (FeCrAl) alloy and have been developed on the basis of irradiation performance during normal and design-basis accident situations. Knowledge gained during previous R\&D via dry volatilization, aqueous chemical separations, and electrochemical operations serves as an excellent starting point for processing accidenttolerant fuel cladding. For example, externally $\mathrm{Cr}$-coated $\mathrm{Zr}$ alloy cladding could be disassembled by shearing with fuel recovery by aqueous dissolution and hull treatment by dry chlorination, as described above. For FeCrAl cladding hulls, both Fe(III) and Al(III) can be chlorinated and volatilized to separate from $\mathrm{Cr}$ and embedded radioactive impurities.

\subsection{Removal of Coating Materials from TRISO-Based Fuels}

Technologies are needed to remove the large amounts of activated graphite from used TRISO fuel assemblies to reduce the amount of material disposed in a deep geological repository. Continuous development of TRISO fuels over the past 70 years has led to their demonstration in high-temperature gas reactors and planned use in very-high-temperature gas reactors of varied design. Interest in this technology has been

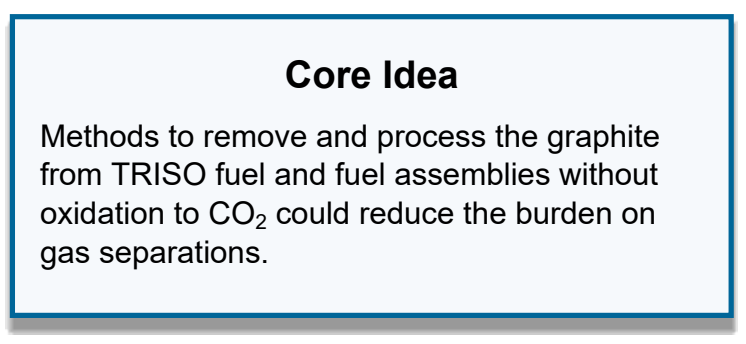
renewed with companies such as BWXT and X-Energy currently producing TRISO fuel and small modular reactor designs such as the Xe-100 scheduled for deployment as early as 2028. Similar to the situation described above for Zircaloy-clad LWR fuel, a substantial mass of TRISO fuel assemblies consists of nonfuel material, primarily carbon. Indeed, carbon makes up most of the mass of the TRISO fuel assemblies. Many of the advanced reactor concepts using TRISO fuel will contain HALEU, and thus 
problems to be expected involve recovery of HALEU from TRISO production batches that do not meet specification or irradiated TRISO fuel that may contain $U$ at higher-than-natural enrichment. The key challenge to developing such technologies is breaking through the carbon and $\mathrm{SiC}$ layers to expose the fuel kernel for subsequent chemical separations operations.

Typical TRISO fuel particles are small ( $1 \mathrm{~mm}$ diameter) spherical pyrolytic carbon/ceramic coated $\mathrm{UO}_{2}$ or UCO fuel kernels (350-600 $\mu \mathrm{m}$ diameter). Figure 2.4 shows the cross section of a TRISO fuel particle. The $\mathrm{UO}_{2}$ or UCO kernel is coated with a pyrocarbon (PyC) buffer layer that creates a void volume to accommodate kernel swelling and $\mathrm{FP}$ gas formation during irradiation. The buffer layer is surrounded by successive layers consisting of an inner PyC layer (IPyC), a silicon carbide layer ( $\mathrm{SiC}$ ), and an outer PyC layer (OPyC). The IPyC provides support for the SiC during irradiation (operation), prevents chlorine reacting with the fuel kernel during $\mathrm{SiC}$ deposition (manufacturing), and retains gaseous FPs. The $\mathrm{SiC}$ layer acts as the pressure vessel and retains gaseous and metal FPs. The OPyC layer lends structural support of the $\mathrm{SiC}$, acts as a FP barrier for particles with defective SiC layers, and provides a bonding surface for further immobilization in a bulk graphite matrix, which acts to both contain the TRISO particles and also as a neutron moderator.

All TRISO fuel concepts require the encapsulation of TRISO particles in a

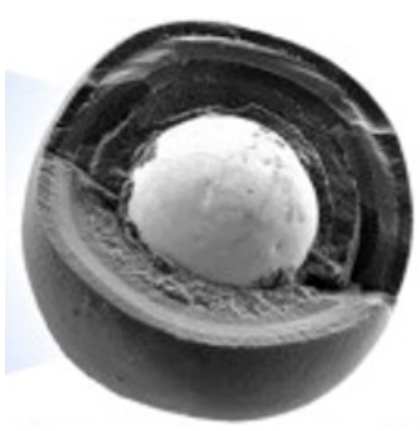

Figure 2.4. Cross section of a TRISO fuel particle showing (from outside to inside) the OPyC, SiC, and IPyC layers and the uranium kernel (Demkowicz 2019, republished with permission of Journal of Nuclear Materials). bulk graphite matrix, the two most common fuel shapes being cylindrical compacts or spherical pebbles. For reactors based on a prismatic core, the TRISO fuel particles are mixed with an organic carbonized binder and graphite, pressed into cylindrical compacts, and sintered to remove any volatile impurities (IAEA 2010). The cylindrical compacts are loaded in graphite sleeves (pseudo fuel rods) and inserted into predrilled hexagonal graphite blocks, which also contain additional holes to accommodate control rods and pathways for coolant flow. Pebble bed reactors use billiard ball-sized (60 $\mathrm{mm}$ diameter) spherical fuel elements. To fabricate them, the TRISO fuel particles are mixed homogeneously with graphite and a carbonized organic binder, pressed into spheres, coated with $5 \mathrm{~mm}$ of fuel-free nuclear-grade carbon, and sintered. Designed to withstand temperatures up to $1,600{ }^{\circ} \mathrm{C}$ without structural damage or any release of FPs (Del Cul et al. 2002), these extremely robust accident-tolerant fuel particles present considerable challenges for recycling.

A typical material balance for one graphite fuel block or fuel element is $0.8 \mathrm{~kg}$ of recoverable fissionable material per $117 \mathrm{~kg}$ total mass of the fuel element. If the compacts are removed from the graphite block before reprocessing, the total mass of carbon will be reduced by $90 \mathrm{~kg}(\sim 81 \%)$ (Del Cul et al. 2002). If the TRISO fuel particles themselves were further removed from the carbon compacts, this step would provide $\sim 90 \%$ overall reduction in the mass of material (mostly carbon) to be disposed or processed. Once removed, TRISO particles would contain a combined $\mathrm{SiC}$ and graphite fraction of $76 \%$, with the remainder of the material being the desired fissionable material and FP wastes. The contaminated bulk carbon presents a severe challenge, since it is present in such large quantities compared to the recoverable fissionable material.

An ORNL report (Del Cul et al. 2002) describes several multistep approaches to processing prismatic TRISO fuel forms, with pebble bed fuel requiring the same processing steps. In the first approach, the graphite fuel blocks are crushed mechanically, and the individual TRISO fuel particles are separated from the graphite. The particles are then burned to remove any filler $\mathrm{C}$ and the OPyC layer to expose the $\mathrm{SiC}$ layer. The outer $\mathrm{SiC}$ layered particles are then crushed and the IPyC layer, buffer carbon layer, and fuel kernel are burned with $\mathrm{O}_{2}$ at $875^{\circ} \mathrm{C}$. The ashes are leached with nitric acid, and the resultant solution can be treated with conventional solvent extraction methods (IAEA 2010; Del Cul et al. 2002). A second 
approach involved burning entire graphite fuel blocks to simplify the process by reducing the number of steps. This approach produces significant quantities of $\mathrm{CO}_{2}$. Because of the presence of ${ }^{14} \mathrm{C}$ from the activation of ${ }^{13} \mathrm{C}$ and the presence of ${ }^{14} \mathrm{~N}$ impurities leading to a ${ }^{14} \mathrm{~N}(\mathrm{n}, \mathrm{p}) \rightarrow{ }^{14} \mathrm{C}$ reaction during irradiation, complete oxidation is undesirable because of the difficulty in recapturing ${ }^{14} \mathrm{CO}_{2}$ without environmental release. Sequestration techniques to capture $\mathrm{CO}_{2}$ are not only expensive, but they would also promote a considerable increase to the mass and volume of the waste compared to the original elemental carbon. Finally, a third approach suggested a grind-leach process, which is used industrially for purification of graphite powder.

Alternatives to the crush-burn-leach approach follow studies conducted by Savannah River National Laboratory on the oxidation of the carbon fuel matrix by nitrate salts, which could reduce the treatment temperature required (Pierce 2017). However, this method also results in formation of ${ }^{14} \mathrm{C}$-contaminated $\mathrm{CO}_{2}$, so capture and disposal of this $\mathrm{CO}_{2}$ would also be an issue that would need to be addressed with this low-temperature oxidation method.

Another alternative approach to removal of bulk carbon from TRISO fuel assemblies without complete oxidation to $\mathrm{CO}_{2}$ is to electrochemically deconsolidate the bulk graphite matrix. During this process, the TRISO compact or pebble will be connected to an electrochemical cell and serve as the anode. When current is applied, the bulk graphite breaks apart into small particles and releases the TRISO fuel particles without breaching the $\mathrm{SiC}$ and carbonaceous layers. This process would allow the separation of the TRISO fuel particles from the deconsolidated bulk graphite matrix by physical means relying on differences in size, density, and shape. This approach is relatively new but has shown promise in literature reports (Tian et al. 2009).

Research is needed to deconsolidate bulk graphite to liberate TRISO particles under controlled conditions. TRISO fuel particles have been found to remain intact during the electrolytic deconsolidation process in concentrated nitric acid. This technique has been used to separate the TRISO particles for postirradiation examination and to examine the escape of FPs from the particles into the compacts (Baldwin et al. 2014; Hunn et al. 2018). Historically, this approach has been thought to be useful for analytical purposes, but due to reaction of strong mineral acids with structural materials, it will be impractical for industrial implementation (Hoogen and Merz 1983). However, recent studies have demonstrated that this approach can utilize more benign supporting electrolytes such as $\mathrm{NH}_{4} \mathrm{NO}_{3}$ and $\mathrm{NaNO}_{3}$ (Tian et al. 2010; Zhang et al. 2016). The current density applied appears to have a great effect on the deconsolidation rate (Xiaotong et al. 2016), with the supporting electrolyte also having an effect but to a much lesser extent. In this way, a holistic approach needs to be identified to select an electrolyte that will be benign to equipment, allow rapid electrolytic deconsolidation, offer compatibility with subsequent processing steps, and minimize formation of ${ }^{14} \mathrm{CO}_{2}$. To achieve this, deconsolidation rates under controlled conditions for a variety of electrolytes with multiple current densities need to be measured and cataloged in the short term. The mechanism of deconsolidation is believed to proceed through a combination of partial oxidation of the carbon along grain boundaries and pressure created along the grain boundaries by intercalation of the anions present in the electrolyte (Lee et al. 2017; Zhang et al. 2019). A more detailed understanding of the mechanism will allow design of improved electrochemical cells and conditions.

After removal of bulk graphite from TRISO particles is completed, breaching the $\mathrm{SiC}$ layer presents a further, and perhaps even more difficult, challenge because of its inert nature. A promising method for this purpose is sonochemistry, which involves acoustic cavitation through formation, growth, and collapse of bubbles in liquid media (Suslick 1990, 2008). Suslick reports the collapsing bubbles induced by cavitation produce

\section{Core Idea}

Efficient means to break through the silicon carbide layer in TRISO fuel particles will allow exposure of the fuel kernel to facilitate separations. 
intense local heating and high pressures in very short times. These localized hot spots reach temperatures of $\approx 5,000 \mathrm{~K}$, pressures approaching $500 \mathrm{~atm}$, and heating and cooling rates $>10^{9} \mathrm{~K} \mathrm{~s}^{-1}$. The large temperature and pressure differentials deliver high-energy heating and microscopic explosive shock waves to a liquid or liquid-solid interface. Cavitation at the surface of a solid in solution induces a deformation in the bubble cavity upon collapse. This deformation reinforces the bubble structure and sends a fast-moving stream of liquid through the cavity at the surface with velocities greater than $100 \mathrm{~m} \mathrm{~s}^{-1}$ (Lauterborn 1984). These impacts leave behind characteristic microscopic pitting on the solid surface that should either penetrate the $\mathrm{SiC}$ shell or destroy it completely during prolonged acoustic irradiation. Figure 2.5(a) shows the formation of such a microjet, and Figure 2.5(b) shows pitting damage of graphite over time due to cavitation at the liquid-solid interface.

(a)

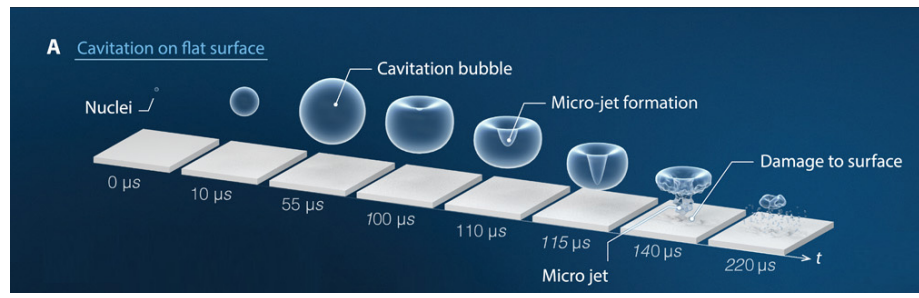

(b)
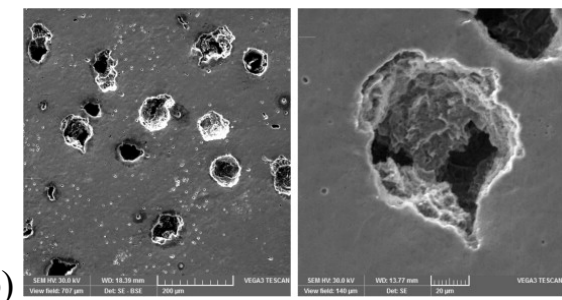

Figure 2.5. (a) Diagram of the formation and collapse of a bubble near a liquid/solid interface creating a microjet (Gonzalez-Avila et al. 2020); (b) SEM image of graphite materials and structural damage (pits) after 15 min cavitation erosion time (Mitelea 2015, republished with permission of Ultrasonics Sonochemistry).

The high energetics of sonochemistry offer a promising approach for future research. The objective here is to penetrate the inner and outer layers of the pyrolytic carbon and the SiC layer of the TRISO fuel particles with localized high pressures and high temperatures (that exceed the $1600{ }^{\circ} \mathrm{C}$ operational window by a factor of 3). The result of using this strategy will expose the desired HALEU uranium fuel kernels, minimize the costly off-gas sequestration by limiting capture to FPs (because $\mathrm{CO}_{2}$ gas does not evolve during sonication), thus establishing a lower cost, significantly reduced volume, simplified one-step process to breach the TRISO coating for subsequent processing.

\subsection{Summary}

Given current US waste disposal regulations, substantial quantities of materials with relatively low radioactivity must be disposed geologically, consuming valuable repository space. Prime examples of this include the $\mathrm{Zr}$ in Zircaloy cladding (from the existing reactor fleet) and the graphite and SiC in TRISObased fuel assemblies (from future reactors). Research is needed to develop methods to separate and decontaminate these materials so that they do not occupy valuable repository space but rather can be either economically disposed of in near-surface LLW disposal facilities or recycled for further use. Furthermore, as the demand for HALEU fuel increases and the fuel form becomes more expensive, it will be important for the United States to pursue novel enabling technologies described here to access enriched $U$ encapsulated in HALEU-based TRISO fuel particles so that it can be recovered for further use. Considerable technical challenges pose barriers to realizing these goals. Research needs are identified for $\mathrm{Zr}$ purification and recycle, reprocessing and component recycle of claddings from accident-tolerant fuels, removing large amounts of graphite from TRISO fuels, deconsolidating graphite, and breaching robust SiC coatings. 


\subsection{References}

Baldwin, C. A.; Hunn, J. D.; Morris, R. N.; Montgomery, F. C.; Silva, C. M.; Demkowicz, P. A. First Elevated-Temperature Performance Testing of Coated Particle Fuel Compacts from the AGR-1 Irradiation Experiment. Nuclear Engineering and Design 2014, 271, 131-41. https://doi.org/10.1016/j.nucengdes.2013.11.021.

Barnes, C.; Vestal, B.; Del Cul, G. D. Recycling Spent Nuclear Fuel Rods Using Sulfur Chloride Reagents. ANS Transactions 2021, 98-99.

Collins, E. D.; Del Cul, G. D.; Rushton, J. E.; Williams, K. A. A Practical Solution to Used Nuclear Fuel Treatment to Enable Sustained Nuclear Energy and Recovery of Vital Materials. ORNL/TM2010/81. Oak Ridge National Laboratory, April 2010.

Collins, E. D.; Del Cul, G. D.; Johnson, J. A.; Hylton, T. D.; Hunt, R. D.; Spencer, B. B.; Bradley, E. C. "Engineering Scale Development/Demonstration of Zirconium Recovery from Used Fuel Cladding." Trans. 2017 ANS Annual Meeting, American Nuclear Society, San Francisco, CA, June 2017.

Del Cul, G. D.; Spencer, B. B.; Forsberg, C. W.; Collins, E. D.; Rickman, W. S. TRISO-Coated Fuel Processing to Support High-Temperature Gas-Cooled Reactors. ORNL/TM-2002/156. Oak Ridge National Laboratory, 2002.

Demkowicz, P. A.; Liu, B.; Hunn, J. D. Coated Particle Fuel: Historical Perspectives and Current Progress. Journal of Nuclear Materials 2019, 515, 434-50.

Gonzalez-Avila, S. R.; Nguyen, D. M.; Arunachalam, S.; Domingues, E. M.; Mishra, H.; Ohl, C.-D. Mitigating Cavitation Erosion Using Biomimetic Gas-Entrapping Microtextured Surfaces (GEMS). Science Advances 2020, 6, 13-22.

Hoogen, N. G.; Merz, E. R. Evaluation of Potential Head-End Procedures for Graphite-Containing Fuel Elements. State-of-the-Art Report on the Progress of Nuclear Fuel Cycle Chemistry, OECD NEA Report 7267. Nuclear Technology 1983, 61(3), 380-87. https://doi.org/10.13182/NT83-A33160.

Hunn, J. D.; Baldwin, C. A.; Montgomery, F. C.; Gerczak, T. J.; Morris, R. N.; Helmreich, G. W.; Demkowicz, P. A.; Harp, J. M.; Stempien, J. D. Initial Examination of Fuel Compacts and TRISO Particles from the US AGR-2 Irradiation Test. Nuclear Engineering and Design 2018, 329 (April 2017), 89-101. https://doi.org/10.1016/j.nucengdes.2017.09.017.

IAEA. High Temperature Gas Cooled Reactor Fuels and Materials. IAEA-TECDOC-1645. International Atomic Energy Agency: Vienna, 2010. https://wwwpub.iaea.org/MTCD/Publications/PDF/TE_1645_CD/PDF/TECDOC_1645.pdf

Lauterborn, W.; Vogel, A. Modern Optical Techniques in Fluid Mechanics. Annual Review of Fluid Mechanics 1984, 16, 223-44.

Lee, J.-H.; Shim, J.-B.; Kim, E.-H.; Yoo, J.-H.; Snyder, C. T.; Lee, J.-H.; Shim, J.-B.; et al. A Feasibility Study for the Development of Alternative Methods to Treat a Spent Triso Fuel. Nuclear Technology 2017, 162(2), 250-58. https://doi.org/10.13182/NT08-A3953.

Mitelea, I.; Bordeasu, I.; Pelle, M.; Crăciunescu, C. Ultrasonic Cavitation Erosion of Nodular Cast Iron with Ferrite-Pearlite Microstructure. Ultrasonics Sonochemistry 2015, 23, 385-90.

Pierce, Robert A. Low Temperature Chemical Processing of Graphite-Clad Nuclear Fuels. US Patent 2017. https://www.osti.gov/servlets/purl/1399896.

Suslick, K. S. Sonochemistry. Science 1990, 247, 1439-45. 
Suslick, K. S.; Flannigan, D. J. Inside a Collapsing Bubble: Sonoluminescence and the Conditions During Cavitation. Annual Review of Physical Chemistry 2008, 59, 659-83.

Tian, L.; Wen, M.; Chen, J. Analysis of Electrochemical Disintegration Process of Graphite Matrix. Electrochimica Acta 2010, 56(2), 985-89. https://doi.org/10.1016/j.electacta.2010.09.050.

Tian, L.; Wen, M.; Li, L.; Chen, J. Disintegration of Graphite Matrix from the Simulative High Temperature Gas-Cooled Reactor Fuel Element by Electrochemical Method. Electrochimica Acta 2009, 54, 7313-17.

Xiaotong, C.; Zhenming, L.; Hongsheng, Z.; Bing, L.; Junguo, Z.; Chunhe, T. The Electric Current Effect on Electrochemical Deconsolidation of Spherical Fuel Elements. International Topical Meeting on High Temperature Reactor Technology, HTR 2017, 767-71.

Zhang, C.; Chen, X.; Liu, B.; Jiao, Z.; Fan, L.; Xu, G.; Wang, T.; He, L; Qi, M; Lu, Z.; Zhao, H.; Yin, Z.; Tang, Y. The Electrochemical Deconsolidation Mechanism of Graphite Matrix in HTGR Spherical Fuel Elements. Journal of Nuclear Materials 2019, 525, 1-6. https://doi.org/10.1016/j.jnucmat.2019.07.023.

Zhang, G.; Wen, M.; Wang, S. Insights into Electrochemical Behavior and Anodic Oxidation Processing of Graphite Matrix in Aqueous Solutions of Sodium Nitrate. Journal of Applied Electrochemistry 2016, 46(12), 1163-76. https://doi.org/10.1007/s10800-016-0999-0. 


\section{FRD 2: Devise Chemistry that Operates Directly on Irradiated Fuel}

\begin{abstract}
This FRD explores methods, other than direct dissolution of the fuel in hot $\mathrm{HNO}_{3}$, for accessing the actinide elements in irradiated fuel for recovery and recycle. Mostly at low technology readiness levels (TRLs), the approaches seek to mitigate issues that arise from the $\mathrm{HNO}_{3}$ dissolution, including management of nitrate, ${ }^{3} \mathrm{H}$, and $I$ and could result in overall simplification of separations. The methods considered for future research include voloxidation of the fuel followed by direct dissolution in process solvents, halide volatility, supercritical fluid extraction, and alkaline-side processing. Also considered in this FRD are separation methods that act directly on the salt in MSRs. Large swaths of unknown chemistry make this FRD particularly ripe with opportunity.
\end{abstract}

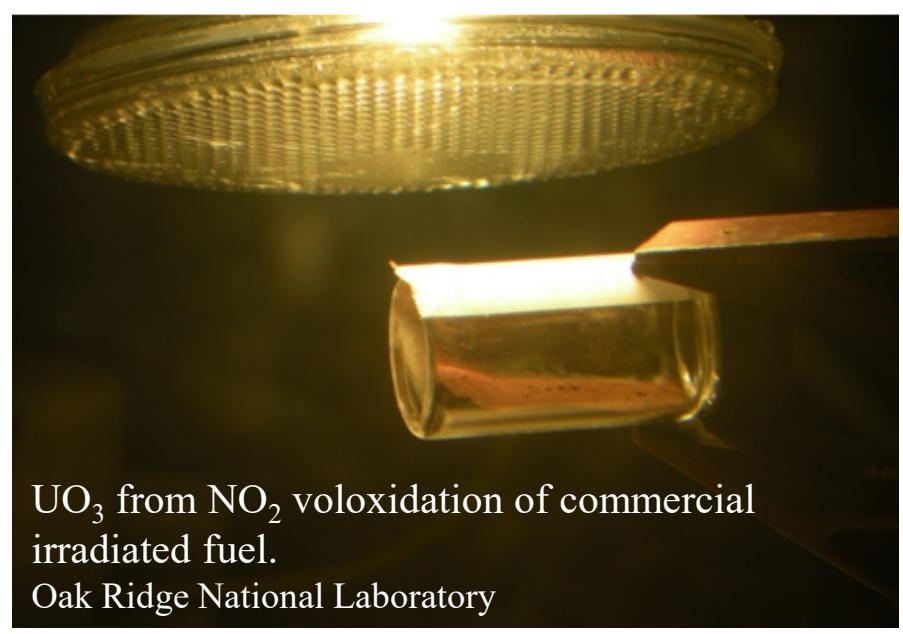

\subsection{Introduction}

As described in Appendix C, the PUREX process represents the current state-of-the-art for recovering $\mathrm{U}$ and $\mathrm{Pu}$ from UNF. The PUREX process is initiated by chopping the fuel rods into small segments and dissolving the fuel in hot $\mathrm{HNO}_{3}$. Although this method of accessing the $\mathrm{U}$ and $\mathrm{Pu}$ for recovery is technologically very mature, it does pose some challenges:

1. Nitrate management. To minimize waste arising from a PUREX reprocessing facility, $\mathrm{HNO}_{3}$ is typically extensively recycled within the facility. This necessitates the inclusion of evaporators within the plant to concentrate solutions and collect the $\mathrm{HNO}_{3}$ rich condensates for reuse. Nitrogen oxide $\left(\mathrm{NO}_{x}\right)$ fumes are evolved during both fuel dissolution and calcination of the HLW stream prior to vitrification; the $\mathrm{NO}_{x}$ is captured for recycle as $\mathrm{HNO}_{3}$ within the reprocessing facility. These nitratemanagement activities add complexity and size to the plant (increased capital cost) and increased operating costs through plant operation and secondary waste management.

2. Tritium management. When the irradiated fuel is dissolved in $\mathrm{HNO}_{3}$, the tritium $\left({ }^{3} \mathrm{H}\right)$ accumulated in the fuel is converted to tritiated water, which is distributed in streams throughout the plant. Existing fuel reprocessing plants located outside of the United States occasionally bleed off the tritiated condensate from evaporators and release the decontaminated (but still tritiated) water to the environment, typically to an ocean. This practice will not be accepted for a fuel reprocessing facility in the United States, especially if the plant is located inland. Instead, tritiated water would need to be managed to likely involve recycle (using large radiological storage systems) and periodic disposal as LLW.

3. Iodine management. Iodine- 129 represents a long-lived $\left(t_{1 / 2}=1.57 \times 10^{7} \mathrm{y}\right)$ radioisotope in irradiated fuel that is mobile in the environment and poses a health hazard because of its propensity to accumulate in the thyroid gland. During $\mathrm{HNO}_{3}$ dissolution of the fuel, most of the iodine is partitioned to the dissolver off-gas stream where it is separated. If not removed another way, the small fraction of iodine remaining in the dissolver solution further partitions between the vessel off-gas during solvent extraction and the HLW raffinate. The iodine in the latter is then mostly released into the off-gas stream 
during calcination of the HLW prior to vitrification. This complex iodine behavior necessitates implementation of complicated iodine abatement systems distributed throughout the plant.

All these complications arise from the fact that the fuel is dissolved in hot $\mathrm{HNO}_{3}$ to put the $\mathrm{U}$ and $\mathrm{Pu}$ into a form (i.e., in solution) that can be exploited for their separation and purification for reuse. Are there alternative methods that can be used to access the $\mathrm{U}, \mathrm{Pu}$, and other actinides that would enable simplification of the plant flowsheet, eliminating or greatly reducing the complications described above? This FRD addresses this question. Five themes are described: (1) voloxidation of the fuel followed by direct dissolution in a process solvent, (2) halide volatility, (3) supercritical fluid extraction, (4) alkaline-side processing, and (5) processing of MSR fuels.

\subsection{Voloxidation and Direct Dissolution in Process Solvents}

Recent literature suggests that nuclear fuel reprocessing flowsheets might be substantially simplified and made appropriate for US application by first performing voloxidation and then directly dissolving the voloxidized fuel in a process solvent consisting of tri- $n$ butyl phosphate (TBP) (Del Cul et al. 2012; Rudisill et al. 2019; Arm. 2021). Two possibilities have been pursued for the voloxidation step: (1) voloxidation in the presence of oxygen or air (referred to as standard voloxidation), or (2) voloxidation using nitrogen dioxide $\left(\mathrm{NO}_{2}\right)$ as oxidant (referred to as advanced voloxidation). Standard voloxidation is conducted at 480-600 ${ }^{\circ} \mathrm{C}$ and results in triuranium octoxide $\left(\mathrm{U}_{3} \mathrm{O}_{8}\right)$ as the product. A primary benefit of performing standard voloxidation is the quantitative release of ${ }^{3} \mathrm{H}$ from the fuel matrix. This allows capture of the ${ }^{3} \mathrm{H}$ on the front end, preventing its distribution throughout the reprocessing facility, and more importantly, allowing management of this radioisotope so that it is not released into the environment. Advanced voloxidation also offers this benefit for ${ }^{3} \mathrm{H}$ management but also operates at a lower temperature, further suggesting other potential avenues to simplify separations flowsheets for iodine and the actinides.

The product of the advanced voloxidation process depends on process conditions employed. Reaction of $\mathrm{UO}_{2}$ with $\mathrm{NO}_{2}$ at $>450{ }^{\circ} \mathrm{C}$ proceeds according to the following reaction:

$$
3 \mathrm{UO}_{2}+2 \mathrm{NO}_{2}(\mathrm{~g}) \rightleftharpoons \mathrm{U}_{3} \mathrm{O}_{8}+2 \mathrm{NO}(\mathrm{g})
$$

Continued treatment of the $\mathrm{U}_{3} \mathrm{O}_{8}$ with $\mathrm{NO}_{2}$ during cooling $\left(200-450{ }^{\circ} \mathrm{C}\right)$ results in the formation of $\mathrm{UO}_{3}$ according to

$$
\mathrm{U}_{3} \mathrm{O}_{8}+\mathrm{NO}_{2}(\mathrm{~g}) \rightleftharpoons 3 \mathrm{UO}_{3}+\mathrm{NO}(\mathrm{g})
$$

Finally, reaction of $\mathrm{UO}_{3}$ with $\mathrm{NO}_{2}$ can continue between 10 and $50{ }^{\circ} \mathrm{C}$ to produce a uranyl nitroso nitrate compound:

$$
\mathrm{UO}_{3}+5 \mathrm{NO}_{2}(\mathrm{~g}) \rightleftharpoons \mathrm{UO}_{2}\left(\mathrm{NO}_{3}\right)_{3} \mathrm{NO}+\mathrm{NO}(\mathrm{g})
$$

So, the range of products that can be formed by voloxidation methods include $\mathrm{U}_{3} \mathrm{O}_{8}, \mathrm{UO}_{3}$, and $\left[\mathrm{UO}_{2}\left(\mathrm{NO}_{3}\right)_{3} \mathrm{NO}\right]$.

As originally envisioned, the $\mathrm{U}_{3} \mathrm{O}_{8}$ produced by standard voloxidation would be dissolved in $\mathrm{HNO}_{3}$, and the resulting solution would be subjected to a PUREX-type solvent extraction flowsheet. However, elimination of the $\mathrm{HNO}_{3}$ dissolution step has recently been suggested by directly dissolving the voloxidized 
fuel into the TBP solvent. This concept has been primarily considered in terms of dissolving the products from advanced voloxidation, namely $\mathrm{UO}_{3}$ or $\left[\mathrm{UO}_{2}\left(\mathrm{NO}_{3}\right)_{3} \mathrm{NO}\right]$ (Figure 3.1).

The dissolution of $\mathrm{UO}_{3}$ and $\left[\mathrm{UO}_{2}\left(\mathrm{NO}_{3}\right)_{3} \mathrm{NO}\right]$ in TBP solvent or TBP solvent saturated with $\mathrm{HNO}_{3}$ has recently been demonstrated (Rudisill et al. 2019). In that study, irradiated fuel simulants prepared by mechanical mixing of actinides and nonradioactive FPs, as well as solid solutions prepared by co-precipitation and calcination of the fuel components were investigated. Effective dissolution of $U$ was achieved under ambient temperature and pressure conditions. Interestingly, $\mathrm{Pu}$ was not efficiently dissolved from the mechanically mixed used fuel simulant, but effective $\mathrm{Pu}$ dissolution was achieved for the solid solutions (which would be expected to be more representative of actual irradiated fuel). Another interesting result from this study was the substantial dissolution of the trivalent lanthanides and actinides (e.g., Am), which is counterintuitive given the low affinity of TBP for the trivalent f-block elements under liquid-

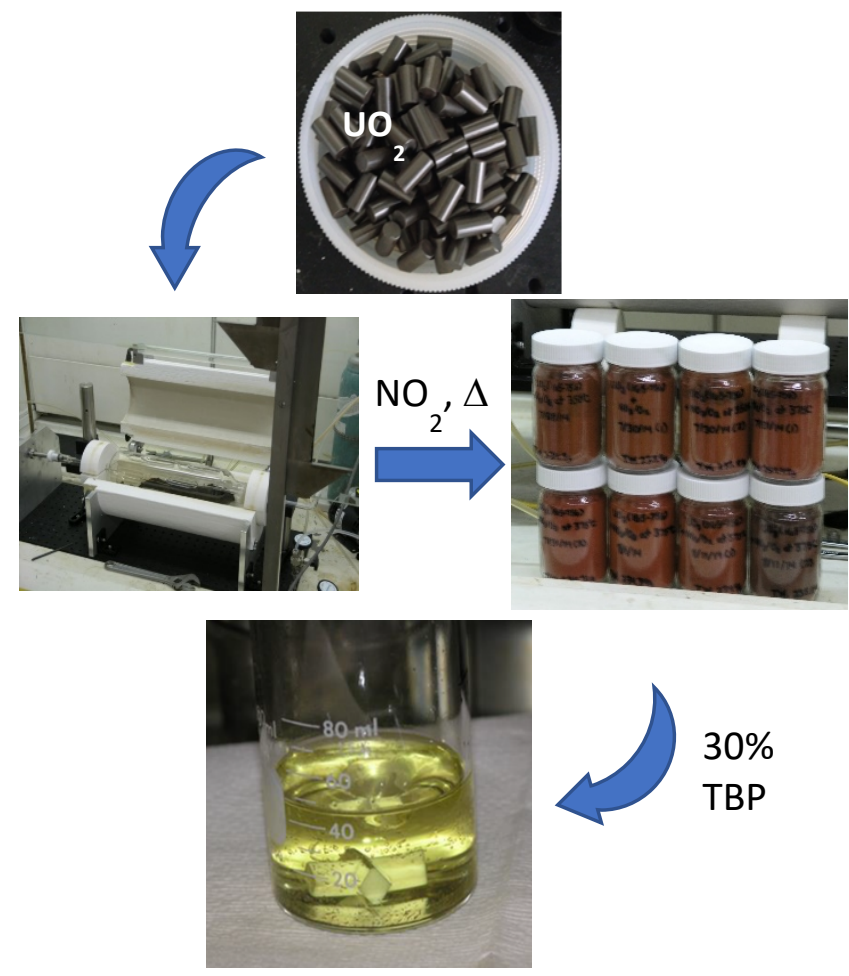

Figure 3.1. Conversion of $\mathrm{UO}_{2}$ pellets to $\mathrm{UO}_{3}$ by voloxidation with $\mathrm{NO}_{2}$, and dissolution of the $\mathrm{UO}_{3}$ in TBP solvent. (Courtesy of ORNL.)

liquid extraction conditions.

The direct dissolution of $\mathrm{UO}_{3}$ and $\left[\mathrm{UO}_{2}\left(\mathrm{NO}_{3}\right)_{3} \mathrm{NO}\right]$ in TBP opens the possibility of pursuing new, simplified process flowsheets for recovering actinides from UNF. But the use of $\mathrm{NO}_{2}$ in the voloxidation step is a potentially complicating feature. This raises the question as to whether a similar approach can be taken with standard voloxidation in which $\mathrm{U}_{3} \mathrm{O}_{8}$ is the primary voloxidation product. The dissolution of $\mathrm{U}_{3} \mathrm{O}_{8}$ in TBP has not been reported. However, dissolution of $\mathrm{UO}_{2}$ in TBP has been reported in the literature (Kulyako, Trofimov et al. 2003). Since both $\mathrm{UO}_{2}$ and $\mathrm{UO}_{3}$ are soluble in $\mathrm{HNO}_{3}$-saturated TBP, it is reasonable to hypothesize that $\mathrm{U}_{3} \mathrm{O}_{8}$ could also be dissolved in this manner.

Research is needed to advance voloxidation chemistry and its coupling to direct solvent dissolution. In the long-term, voloxidation improvements could include achieving more complete volatilization of gaseous components and use of alternative oxidants and conditions. Coupling the voloxidation step with various subsequent separation steps such as direct solvent dissolution has the potential to reduce steps and space (process intensification). Based on the promising results reported in the literature, several more immediate research needs are evident to advance the voloxidation/direct solvent dissolution concept. There are four critical research needs:

1. Characterizing the distribution of the various UNF components (particularly iodine, ${ }^{14} \mathrm{C}$, and ${ }^{3} \mathrm{H}$ ) during $\mathrm{NO}_{2}$ voloxidation

2. Determining the degree of dissolution of irradiated fuel components when voloxidized fuel is dissolved in TBP-based and other potential process solvents 
3. Developing and demonstrating process flowsheets based on voloxidized fuel directly dissolved in the process solvent

4. Addressing engineering challenges unique to the voloxidation/direct solvent dissolution concept (e.g., dissolver design, clarification from undissolved solids, etc.)

\subsection{Halide Volatility}

Exploiting differences in the vapor pressure properties of metal halides in the reprocessing of irradiated fuel opens the door to a family of separation approaches operating directly on used fuel, as reviewed by Tripp et al. (2011). A variety of methods can be considered, but they generally can be divided into those involving fluorination and those involving chlorination.

\section{Core Idea}

Compact fuel separation systems can be achieved by exploiting differences in the volatility of metal halide species at different temperatures.

\subsubsection{Fluorination}

The volatile properties of $\mathrm{UF}_{6}$ have been exploited at the front end of the nuclear fuel cycle since the Manhattan Project in the 1940s. Fluoride volatility methods have also been considered for reprocessing irradiated nuclear fuel, but such an application has yet to be realized at an industrial scale for commercial nuclear fuel. The potential to achieve separations lies in the differences in the volatility of the fluoride compounds of the chemical elements contained in irradiated nuclear fuel (see Table 3.1). Substantial work has been conducted over the last two decades on fluorination of the fuel with elemental fluorine $\left(\mathrm{F}_{2}\right)$ in flame fluorination devices (Kani et al. 2009; Uhlir and Marecek 2009). In the flame fluorinator, the $\mathrm{F}_{2}$ indiscriminately converts all the irradiated fuel components into their fluorides. The volatile fluoride species exit the fluorinator, and the nonvolatile species remain behind as ash. Depending on the conditions, the actinide elements $\mathrm{U}, \mathrm{Np}$, and $\mathrm{Pu}$ can all form volatile hexafluoride species. Different strategies have been proposed for separating $\mathrm{U}, \mathrm{Pu}$, and $\mathrm{Np}$.

In the FLUOREX (fluoride volatility solvent extraction) process, Japanese researchers have proposed a hybrid system in which the $U$ is separated during fluorination, but the fluorinator is operated in a manner that most of the $\mathrm{Pu}$ ends up in the nonvolatile $\mathrm{PuF}_{4}$ form (Kani, Sasahara et al. 2009). The fate of Np in this process is not clear, but it is likely to be converted to $\mathrm{NpF}_{6}$ that exits the fluorinator with the $\mathrm{UF}_{6}$. The $\mathrm{Pu}$ and other nonvolatile fluorides are converted to oxides by pyrohydrolysis. This small amount of material can then be dissolved in $\mathrm{HNO}_{3}$ and processed via solvent extraction to recover the Pu. Researchers in the Czech Republic have been pursuing a completely dry process (Uhlir and Marecek 2009). In this case, sufficient free $\mathrm{F}_{2}$ is present during the fluorination step to maintain $\mathrm{Pu}$ in the thermally unstable (yet volatile) form of $\mathrm{PuF}_{6}$, and the $\mathrm{U}, \mathrm{Np}$, and $\mathrm{Pu}$ are collected together. The $\mathrm{Pu}$ is then separated by thermal decomposition to nonvolatile $\mathrm{PuF}_{4}$. Neptunium can be separated by sorption of $\mathrm{NpF}_{6}$ on $\mathrm{MgF}_{2}$.

Nitrogen trifluoride $\left(\mathrm{NF}_{3}\right)$ has been suggested as an alternative fluorinating agent (Scheele et al. 2012). This fluorinating reagent offers two key advantages over $\mathrm{F}_{2}$. First, $\mathrm{NF}_{3}$ is relatively nonhazardous, so it is easier to handle than the highly corrosive $F_{2}$. Second, it is less reactive than $F_{2}$, which offers the possibility of achieving some separations by controlling the reaction conditions (Figure 3.2). For example, $\mathrm{NF}_{3}$ is not sufficiently powerful to convert $\mathrm{Pu}$ and $\mathrm{Rh}$ into volatile forms, so these elements will remain in the nonvolatile fluoride ash and will not carry over into the $U$ fraction. This offers a significant nonproliferation benefit if $U$ recycling is the objective. Bromine trifluoride has also been studied as a fluorinating agent more selective than $\mathrm{F}_{2}$, but it is a highly toxic material and more difficult to handle than $\mathrm{NF}_{3}$. 
Table 3.1. Relative Volatilities of Fluorides of Significance to Irradiated Fuel Reprocessing

\begin{tabular}{lll}
\hline Highly volatile & Volatile & Nonvolatile \\
\hline $\mathrm{Kr}$ & $\mathrm{IF}_{7}$ & $\mathrm{AmF}_{4}$ \\
$\mathrm{CF}$ & $\mathrm{MoF}_{6}$ & $\mathrm{RhF}_{3}$ \\
$\mathrm{Xe}$ & $\mathrm{NpF}_{6}$ & $\mathrm{SnF}_{4}$ \\
$\mathrm{TeF}_{6}$ & $\mathrm{TcF}_{6}$ & $\mathrm{ZrF}_{4}$ \\
$\mathrm{SeF}_{6}$ & $\mathrm{UF}_{6}$ & $\mathrm{PuF}_{4}$ \\
& $\mathrm{PuF}_{6}$ & $\mathrm{CsF}$ \\
& $\mathrm{IF}_{5}$ & $\mathrm{RbF}$ \\
& $\mathrm{SbF}_{5}$ & $\mathrm{UF}_{4}$ \\
& $\mathrm{NbF}_{5}$ & $\mathrm{AmF}_{3}$ \\
& $\mathrm{RuF}_{5}$ & $\mathrm{CmF}_{3}$ \\
& $\mathrm{RuF}_{6}$ & $\mathrm{YF}_{3}$ \\
& $\mathrm{RhF}_{5}$ & $\mathrm{BaF}_{2}$ \\
& $\mathrm{RhF}_{6}$ & $\mathrm{EuF}_{3}$ \\
& & $\mathrm{GdF}_{3}$ \\
& & $\mathrm{CeF}_{4}$ \\
& & $\mathrm{CeF}_{3}$ \\
& & $\mathrm{PmF}_{3}$ \\
& & $\mathrm{SmF}_{3}$ \\
& & $\mathrm{SrF}_{2}$ \\
\hline
\end{tabular}

Note: Adapted from Uhlir and Marecek (2009).

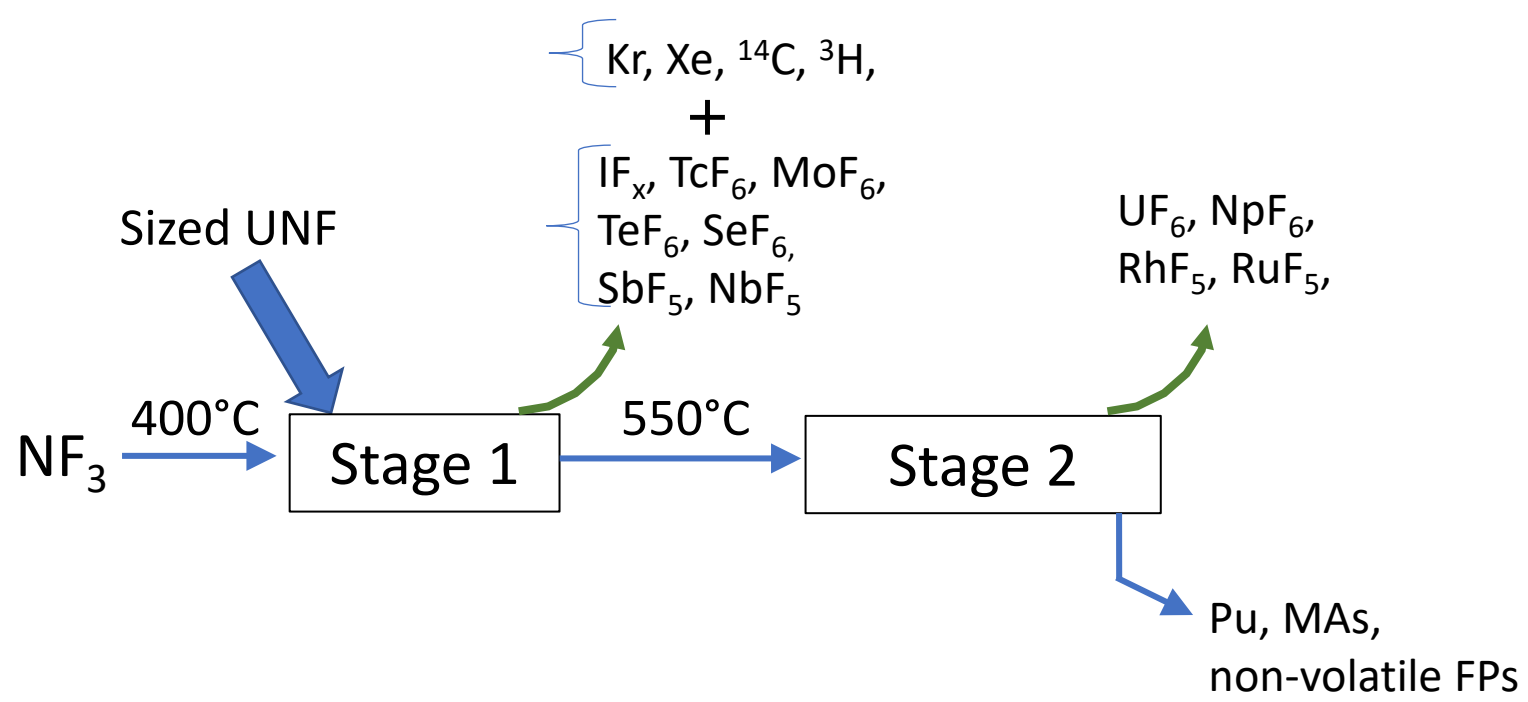

Figure 3.2. Separation scheme exploiting selective fluorination properties of $\mathrm{NF}_{3}$. (Courtesy of ORNL.)

The $\mathrm{UF}_{6}$ recovered from irradiated fuel can be purified by a sequence of sorption/desorption cycles.

Uranium hexafluoride can reversibly sorb onto $\mathrm{NaF}$ based on the following reaction (Long 1978):

$$
\mathrm{Na}_{2} \mathrm{UF}_{8} \rightleftharpoons 2 \mathrm{NaF}+\mathrm{UF}_{6}
$$

Fluorine is added to the system to minimize the contribution of the competing reaction:

$$
\mathrm{Na}_{2} \mathrm{UF}_{8} \rightleftharpoons \mathrm{Na}_{2} \mathrm{UF}_{6}+\mathrm{F}_{2}
$$


Uranium, neptunium, and plutonium hexafluorides all sorb onto $\mathrm{NaF}$ at $100{ }^{\circ} \mathrm{C}$ (Uhlir and Marecek 2009). The uranium and neptunium hexafluorides can be desorbed from $\mathrm{NaF}$ by raising the temperature to $400{ }^{\circ} \mathrm{C}$ under a flow of $\mathrm{F}_{2}$. However, $\mathrm{Pu}$ will be retained on the $\mathrm{NaF}$ bed by formation of $\mathrm{PuF}_{4} \cdot 3 \mathrm{NaF}$. Partial separation of $\mathrm{Np}$ from $\mathrm{U}$ can be achieved by sorption of $\mathrm{NpF}_{6}$ onto $\mathrm{MgF}_{2}$, but a complete scheme for separating $\mathrm{U}$ and $\mathrm{Np}$ remains to be developed. Molybdenum, Tc, I, and $\mathrm{Sb}$ follow the $\mathrm{U}$ past the $\mathrm{NaF}$ and $\mathrm{MgF}_{2}$ sorption beds. Separation of these elements from the $\mathrm{U}$ may be possible with fractional distillation, but this technology is not fully developed.

Research is needed to develop separation schemes and fluorination agents to advance the fluoride volatility method. Many of the basic concepts are known, especially regarding formation and volatilization of fluorinated species. However, new chemistry can still change this landscape, particularly achieving downstream separations. Innovative methods of controlling the volatility of fluorinated species has the potential to simplify the separation schemes. There are five critical research needs:

1. Performing scaled demonstrations of separation schemes based on $\mathrm{NF}_{3}$

2. Developing and demonstrating strategies to separate $\mathrm{Np}$ from $\mathrm{U}$

3. Developing and demonstrating methods for final purification of $\mathrm{UF}_{6}$ from the Mo, Tc, $\mathrm{I}$, and $\mathrm{Sb}$ impurities

4. Developing strategies to recycle $\mathrm{Pu}$ in the context of fluorination schemes in pursuit of a fully closed fuel cycle

5. Addressing challenges associated with material of construction of plant equipment due to the very corrosive nature of the fluorination reagents

\subsubsection{Chlorination}

Chloride volatility methods separate compounds by chlorinating the feedstock, if needed, then separating chloride compounds by exploiting differences in their vapor pressures. In some schemes, the desired product is volatilized out of a mixture and collected. In other approaches, impurities are removed from the desire product by volatilization. Chloride volatilization has been performed for decades as a tool for metal separations in general, but key gaps in our understanding of the chemistry and properties of chlorination and corresponding separations as applied to actinides and FPs still present major challenges.

In 1943, Brown and Seaborg filed a patent for separating U, Pu, and FPs under a stream of $\mathrm{Cl}_{2}$ (Brown and Seaborg 1959). In the 1960s, the chlorination of actinide oxides and purification of the resulting chlorides was investigated at ORNL (Goode 1964). Fundamental thermodynamic calculations and laboratory studies were performed at ORNL using $\mathrm{Cl}_{2}, \mathrm{CCl}_{4}, \mathrm{COCl}_{2}$, and $\mathrm{CCl}_{4}-\mathrm{Cl}_{2}$ mixtures. When chlorinating oxides, the presence of an oxygen getter, such as carbon, was essential in forming the chlorides. Warren and Farris were able to completely volatilize $\mathrm{U}$ and $\mathrm{Pu}$ from $\mathrm{UO}_{2}-\mathrm{PuO}_{2}$ mixtures (Warren and Ferris 1966). Goode (1964) performed chloride volatility experiments on $\mathrm{UO}_{2}$ samples spiked with irradiated $\mathrm{UO}_{2}$ using $\mathrm{CCl}_{4}-$ $\mathrm{Cl}_{2}$ gas mixtures. He observed that "separation was difficult from those elements having vapor pressures similar to that of the uranium" and "methods of control of the oxidation state of the uranium appear critical to prevent losses." During this time the zirconium extraction (ZIRCEX) process was also developed, which completely chlorinates UNF and cladding with $\mathrm{HCl}$ then $\mathrm{CCl}_{4}$ in a single stage (Gens et al. 1961). The chlorination products are all volatilized together, and uranium chloride $\left(\mathrm{UCl}_{3}\right)$ is condensed at $300{ }^{\circ} \mathrm{C}$.

Argonne National Laboratory also demonstrated a volatility process for U alloys in the 1960 s, which was called the fluid-bed fluoride volatility process (Chilenskas and Turner 1968). In the first step, the chlorinated alloys and aluminum and zirconium are removed as volatile chlorides. In the second step, $\mathrm{UCl}_{3}$ created in step one is fluorinated with $\mathrm{HF}$ to form $\mathrm{UF}_{4}$ and avoid the formation of $\mathrm{Cl}_{2}$ and chloroflourides. 
In the last step, the $\mathrm{UF}_{4}$ is reacted with fluorine gas to form $\mathrm{UF}_{6}$, which is volatilized from the remaining material.

In India during the 1960s, chloride volatility processes were also being developed (Hariharan et al. 1970). Similar to the work at $\mathrm{ORNL}, \mathrm{U}$ and $\mathrm{Pu}$ oxides were chlorinated using $\mathrm{Cl}_{2}$ saturated with $\mathrm{CCl}_{4}$. However, sodium chloride $(\mathrm{NaCl})$ beds were used to sorb volatilized $\mathrm{UCl}_{3}$ nearly completely at $250-300{ }^{\circ} \mathrm{C}$. The researchers attributed this retention of uranium chlorides to the formation of the $\mathrm{Na}_{2} \mathrm{UCl}_{6}$ complex. This illustrates one of the possible interactions that could potentially alter the vapor pressure of individual species when mixed with other chlorides. Unfortunately, the analysis by the researchers at the time was limited, and a clear understanding of the chemistry is still not established.

In 1990s, Japanese researchers examined the chlorination of denitrified high-level liquid waste from aqueous processing, and volatile chlorides were captured in a cold trap (Kurata 2000). This was treated more as a conversion/head end process for molten salt electrorefining rather than a separation process. More recently, European researchers have been investigating chloride volatility for recovering actinide metals deposited on aluminum cathodes during electrorefining (Cassayre et al. 2011; Soucek et al. 2014). This is done by distilling residual salt on the cathode, chlorinating the actinide and aluminum metals with $\mathrm{Cl}_{2}$ gas at $150{ }^{\circ} \mathrm{C}$, and then sublimating under an argon flow at $350-400{ }^{\circ} \mathrm{C}$. This process is effective at removing highly volatile $\mathrm{AlCl}_{3}$ (sublimes at $180{ }^{\circ} \mathrm{C}$ ), but it is designed to use the initial purification step of electrorefining.

In the last 5 years, chloride volatility has been applied to purifying $\mathrm{CeCl}_{3}$ (Okabe et al. 2021). In this work, uranium metal was successfully hydrided, chlorinated, and volatilized both as a pure metal and as an impurity in Ce metal (see Figure 3.3). To achieve complete volatility of $\mathrm{U}, \mathrm{Cl}_{2}$ of sufficient purity needed to be used. Furthermore, the same degree of separation was achieved when recycling the $\mathrm{Cl}_{2}$, resulting in one-third of the once-through amount of $\mathrm{Cl}_{2}$ used.

Paralleling the case with fluorination, technology gaps in the development of separation schemes and chlorination agents raise the following needs:

- "Control of the oxidation state of the uranium . . to to prevent losses" (Goode 1964)

- Improve thermal control to separate "those elements having vapor pressures similar to that of the uranium" (Goode 1964)

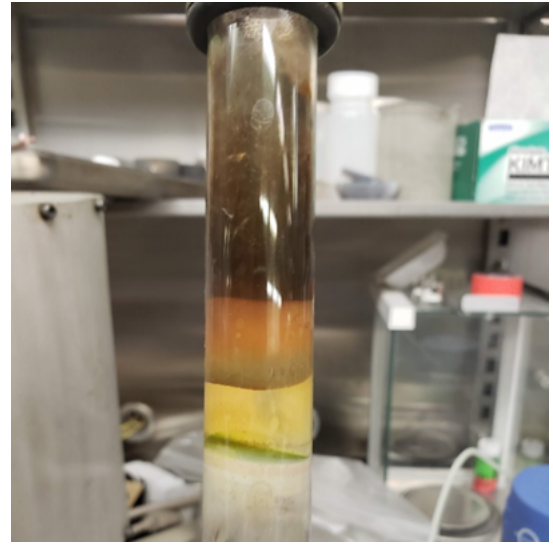

Figure 3.3. Uranium chloride deposits on glass tube after chlorinating and volatilizing uranium metal under flowing $\mathrm{Cl}_{2}$ gas at $500{ }^{\circ} \mathrm{C}$ (Okabe 2020).

- Improve our understanding of chloride complexes and their behavior (e.g., vapor pressures) that may form in the solid and gas phases (Hariharan 1970)

- Improve our understanding of the effects of impurities in the chlorinating gas on the chemistry of the chlorides and their behavior (e.g., vapor pressures)

- Develop waste minimization strategies (e.g., recycle of chlorinating gas) to reduce the need for caustic scrubbers and sorbents

To address these gaps, the following research is needed:

1. Developing and/or applying instrumentation to the measure the composition of the gas phase over a salt mixture 
2. Fully developing chlorination methods and corresponding separation schemes based on chloride volatility, including separation schemes for achieving specific goals (e.g., $\mathrm{U}$ only recycle, U/Pu recycle, etc.)

3. Addressing challenges associated with material of construction of plant equipment due to the very corrosive nature of the chlorination reagents

\subsection{Supercritical Fluid Extraction}

As discussed in Appendix C, the industry standard for reprocessing of irradiated nuclear fuel involves dissolving the fuel in hot $\mathrm{HNO}_{3}$ followed by solvent extraction with TBP in an aliphatic diluent (i.e., the PUREX process). Supercritical fluid extraction (SFE) is a mature industrial technology used in industry broadly (e.g., in the decaffeination of coffee). However, the application of SFE in nuclear reprocessing is

\section{Core Idea}

Supercritical fluid extraction can eliminate the use of combustible diluents, reduce plant footprint, and drastically decrease secondary waste streams. comparatively unexplored. Supercritical $\mathrm{CO}_{2}\left(\mathrm{sc}-\mathrm{CO}_{2}\right)$ has been proposed as a green solvent for industrial fuel reprocessing because of the lack of volatile organic compounds and the generation of less secondary waste (Wai 2006). Additionally, sc- $\mathrm{CO}_{2}$ has been predicted to reduce equipment cost and the amount of acidic waste, and it is inherently more resistant to fires and explosions. Fundamentally, sc- $\mathrm{CO}_{2}$ extraction operates in a very similar manner to existing solvent extraction schemes for the nuclear fuel cycle wherein an extractant is dissolved in an aliphatic diluent, except the diluent in this case is sc- $\mathrm{CO}_{2}$. Furthermore, the product can be captured by bleeding off the $\mathrm{CO}_{2}$ for recycle, leaving a pure extractant loaded with actinide elements, which can then be stripped to recover the product.

In nuclear reprocessing, sc- $\mathrm{CO}_{2}$ has the potential to operate with two different head-end processes: (1) a "wet" process in which the fuel is first dissolved in hot $\mathrm{HNO}_{3}$ similar to conventional processing and then extracted from the aqueous phase with the sc- $\mathrm{CO}_{2}$ phase; or (2) a "dry" process in which a sc- $\mathrm{CO}_{2} /$ extractant mixture is loaded with $\mathrm{HNO}_{3}$ and then used to directly dissolve the fuel (Wai 2006). The dry process generally sees a greater extraction efficiency; however, due to the absence of water, trivalent $f$-elements are coextracted to a degree that may make achieving desirable separation factors more difficult.

The extraction of actinide elements into sc- $\mathrm{CO}_{2}$ containing TBP has been demonstrated in the US (Mincher 2016), as well as internationally. The two most developed processes to date are the Super-DIREX (supercritical fluid direct extraction) process from Japan (Enokida et al. 2007), and the RELICT (reprocessing by liquid $\mathrm{CO}_{2}$ treatment) process from Russia (Samsonov et al. 2011). Both the Super-DIREX and RELICT process follow a dry head-end process. Although the majority of the work on the SuperDIREX and RELICT process focused on the extraction of surrogate $\mathrm{UO}_{2}$ and mixed-oxide (MOX) fuel, a study did demonstrate the head-end process on irradiated fuel using both supercritical and liquid $\mathrm{CO}_{2}$. Extraction into sc- $\mathrm{CO}_{2}$ was shown to be more effective than into liquid $\mathrm{CO}_{2}$. Coextraction of the trivalent $f$-elements did occur to a degree, but a preliminary separation from high-radiological dose FPs such as Cs and $\mathrm{Sr}$ (and to a lesser degree the trivalent lanthanides) may be achievable during the dissolution stage if a dry pathway is pursued.

China has also begun investigating sc- $\mathrm{CO}_{2}$ processes with much of the effort focused in the past 10 years (Zhu 2011). The suggested application for much of the Chinese work is forward thinking with direct recovery from TRISO fuel kernels being proposed. Presumably this would occur after electrolytic deconsolidation or some other means for removal of the bulk graphite matrix. 
Supercritial- $\mathrm{CO}_{2}$ came close to industrial implementation for the recovery of enriched $\mathrm{U}$ from incinerator ashes by AREVA (Koegler 2010). The decision to not move forward with the technology appears to have been due to the cost of increasing the technology readiness level (TRL) to industrial implementation rather than inherent flaws in the concept. Thus, $\mathrm{R} \& \mathrm{D}$ to raise the TRL for $\mathrm{sc}-\mathrm{CO}_{2}$ extraction presents an opportunity.

Several critical technical challenges exist for realizing a TBP-based $\mathrm{sc}-\mathrm{CO}_{2}$ process:

1. Investigating the dissolution of the actinide elements directly into $\mathrm{sc}-\mathrm{CO}_{2} / \mathrm{TBP}$ after voloxidation of LWR fuel

2. Developing and demonstrating flowsheets at the laboratory scale for the recovery of $\mathrm{U}, \mathrm{Np}$, and $\mathrm{Pu}$

3. Optimizing sc- $-\mathrm{CO}_{2}$ pressure and temperature with respect to $\mathrm{TBP}$ and $\mathrm{HNO}_{3}$ during loading and stripping steps

4. Developing flowsheet modeling software (including collection of thermodynamic data) for supercritical $\mathrm{CO}_{2}$ systems

5. Designing and testing and qualification of contacting equipment which are robust to supercritical fluids in high-radiation environments

6. Understanding the spectroscopic signatures of relevant actinides and FPs in the sc- $\mathrm{CO}_{2}$ phase to enable online monitoring.

General research needs for realizing the potential for SFE approaches to direct actinide separations from UNF include finding supercritical fluid-extractant combinations that effect actinide dissolution coupled with efficient separation schemes. Given the lower TRL of SFE compared to conventional solvent extraction, opportunities arise to negate unwanted attributes of TBP-based processes. These include formation of radiolysis products detrimental to separation and subsequent processing steps and production of difficult-to-vitrify phosphorus-containing secondary waste. The monoamide extractants that are completely incinerable have proven to be stable to radiolysis and provide adequate separation factors in conventional solvent extraction. The solubility of several monoamides in sc- $\mathrm{CO}_{2}$ has been examined and are sufficiently high to be practical as a solvent for nuclear processing (Pitchaiah et al. 2019), but the application of monoamides in sc- $\mathrm{CO}_{2}$ for the reprocessing of advanced reactor (and LWR) fuel is essentially unexplored. The choice of potential extractants for SFE quite generally is open-ended. FRD 5 examines coordination-based separation approaches, suggesting many more possible ways to exploit SFE.

\subsection{Alkaline-Side Processing}

Reprocessing of UNF based on dissolution in carbonate-peroxide media offers a paradigm shift from the traditional $\mathrm{HNO}_{3}$ dissolution/PUREX approach (Soderquist et al. 2011). This approach offers several potential advantages.

\section{Core Idea}

Enable simplified separation schemes by dissolving the fuel in alkaline media instead of hot nitric acid.

1. Simplified off-gas management. Dissolving $\mathrm{UNF}$ in $\mathrm{HNO}_{3}$ results in the evolution of copious amounts of $\mathrm{NO}_{x}$ that must be managed. Although technologies exist for managing $\mathrm{NO}_{x}$ within nuclear fuel reprocessing facilities, these operations result in substantial capital and operating costs. Under the hot $\mathrm{HNO}_{3}$ dissolution conditions iodine is substantially volatilized, complicating the dissolver off-gas system. Iodine is not expected to be volatile under alkaline conditions.

2. Dissolution under ambient conditions. The dissolution of UNF in carbonate-peroxide media proceeds under ambient conditions of temperature and pressure, offering the possibility of simplified plant design and reduced operating costs. 
3. Reduction in organic solvent use. In principle, the chemical form of uranium (e.g., $\left.\left[\mathrm{UO}_{2}\left(\mathrm{CO}_{3}\right)_{3}\right]^{4-}\right)$ obtained by dissolving under alkaline conditions offers the possibility of simplified schemes for separating it from $\mathrm{Pu}$ and other fuel components, including those that do not involve solvent extraction. If recovery of other actinides $(\mathrm{Pu}, \mathrm{Np}, \mathrm{Am}, \mathrm{Cm})$ is desired, this could be achieved by dissolving the very small amount of alkaline-insoluble material containing these elements in $\mathrm{HNO}_{3}$ and performing solvent extraction; the $\mathrm{HNO}_{3}$ and solvent use under such a scenario would be two orders of magnitude less than in a conventional PUREX plant.

Carbonate-peroxide dissolution and partitioning of the fuel components has been tested on irradiated commercial nuclear fuel with promising results (Soderquist 2011), but developing alkaline-side separation schemes remains a ripe area of research. A full accounting of the fuel components is required; the behavior of ${ }^{14} \mathrm{C}$ is especially an open question.

Figure 3.4 presents an exemplary UNF separation concept based on carbonate-peroxide dissolution. Although $\mathrm{UO}_{2}$ fuel can be dissolved directly in carbonate-peroxide media, the scheme presented in Figure 3.3 begins with voloxidation of the fuel. As described above, the intended benefit of performing voloxidation is to remove ${ }^{3} \mathrm{H}$ up front so it can be captured and managed more easily. The voloxidized fuel is dissolved in a solution containing ammonium carbonate $\left[\left(\mathrm{NH}_{4}\right)_{2} \mathrm{CO}_{3}\right]$ and hydrogen peroxide $\left(\mathrm{H}_{2} \mathrm{O}_{2}\right)$. The dissolution process proceeds under ambient conditions. The dissolved fuel solution is heated to decompose the $\mathrm{H}_{2} \mathrm{O}_{2}$ and drive off the $\left(\mathrm{NH}_{4}\right)_{2} \mathrm{CO}_{3}$ until the TRUs and most of the FPs precipitate. The solution, containing primarily $\left[\mathrm{UO}_{2}\left(\mathrm{CO}_{3}\right)_{3}\right]^{4-}$, is passed through a cation exchanger to remove Cs. Crystalline silicotitanate (Anthony 1994) is an attractive option for this step because it is highly selective for Cs, and the Cs-loaded crystalline silicotitanate can be packaged for direct disposal. Following Cs removal, the U-containing solution is concentrated and cooled to form crystalline $\left(\mathrm{NH}_{4}\right)_{4} \mathrm{UO}_{2}\left(\mathrm{CO}_{3}\right)_{3}$, which can be calcined to yield a uranium oxide product. The solid fraction obtained after removal of the $\mathrm{H}_{2} \mathrm{O}_{2}$ and $\left(\mathrm{NH}_{4}\right)_{2} \mathrm{CO}_{3}$ can be either immobilized and disposed of as HLW or dissolved and processed through appropriate solvent extraction methods to yield actinide products suitable for further use in the nuclear fuel cycle.

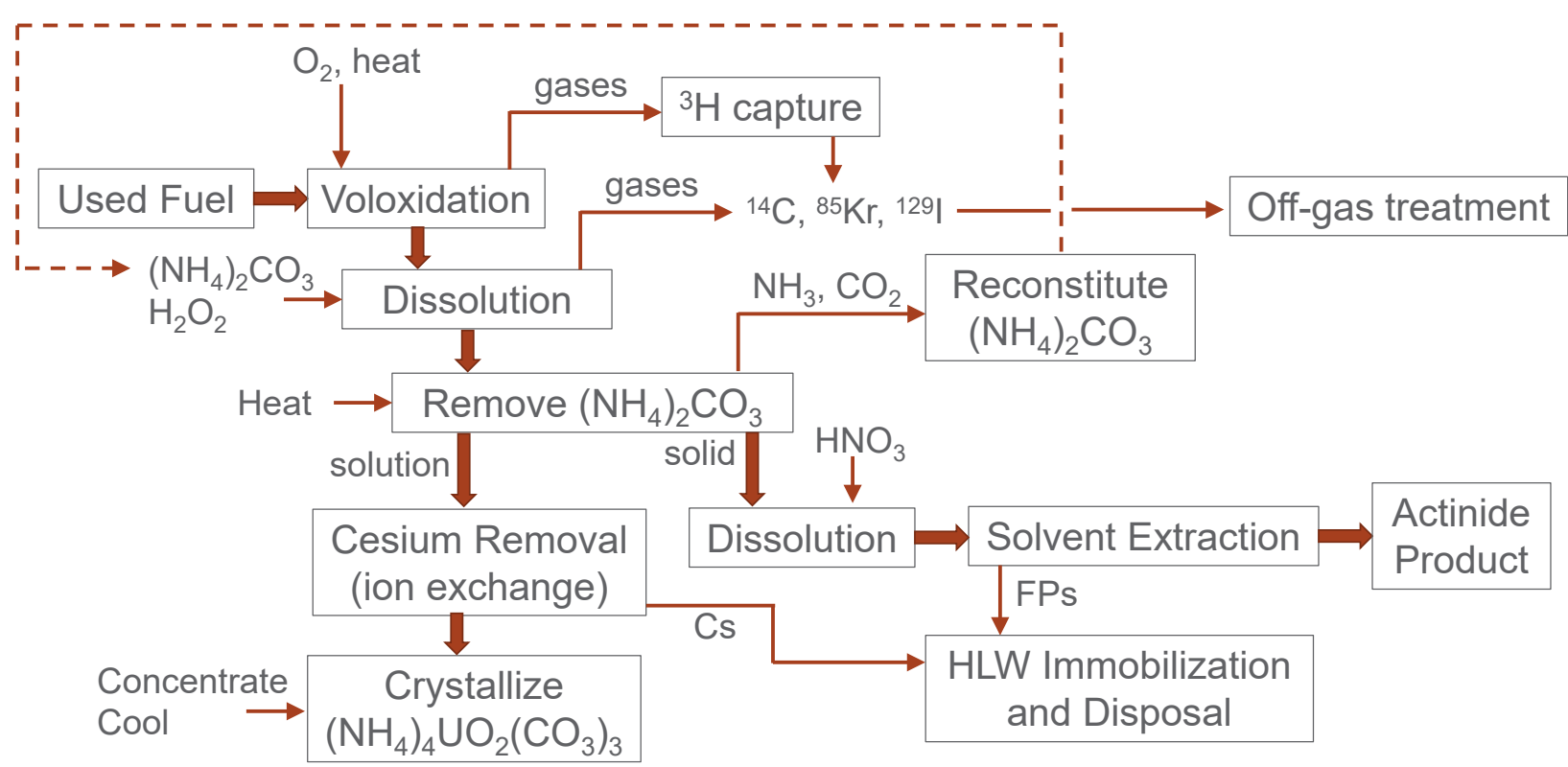

Figure 3.4. Schematic representation of a used fuel separation scheme based on carbonate-peroxide dissolution of the fuel. (Courtesy of PNNL.) 
The scheme shown in Figure 3.4 includes substantial knowledge gaps. One of the most significant of these is the fate of ${ }^{14} \mathrm{C}$. Voloxidation is known to release $\sim 20 \%$ of the ${ }^{14} \mathrm{C}$ from the fuel matrix (Stone and Johnson 1978), so $\sim 80 \%$ of the ${ }^{14} \mathrm{C}$ would be present when the fuel is dissolved in the carbonate-peroxide medium. It can be hypothesized that much of this ${ }^{14} \mathrm{C}$ will follow into the reconstituted $\left(\mathrm{NH}_{4}\right)_{2} \mathrm{CO}_{3}$ stream, which is recycled within the process flowsheet. This would presumably result in a buildup of ${ }^{14} \mathrm{C}$ in the recycled $\left(\mathrm{NH}_{4}\right)_{2} \mathrm{CO}_{3}$. This possibility must be investigated, and approaches to mitigate the impact of ${ }^{14} \mathrm{C}$ buildup must be devised.

Iodine is expected to remain mostly in solution up through the $\left(\mathrm{NH}_{4}\right)_{4} \mathrm{UO}_{2}\left(\mathrm{CO}_{3}\right)_{3}$ crystallization step. The mother liquor resulting from this precipitation step might be recycled back to the front end of the process, or it might be immobilized for disposal. Specific options for processing the mother liquor may depend on the iodine behavior in the upstream processing steps. Understanding the fate of most of the fuel components during alkaline processing remains a challenge and must be more thoroughly examined. For example, the study performed on irradiated UNF indicated a substantial amount of the $\mathrm{Sr}$ followed Cs (Soderquist et al. 2011), which was unexpected based on classical inorganic chemistry principles.

Major research opportunities exist for simplifying separations through the development of alkaline-side UNF reprocessing. Compared with the aqueous chemistry developed for UNF reprocessing from acid solutions, we have hardly scratched the surface of alkaline-side separations chemistry. Research needs include the following:

1. Understanding and improving the fuel dissolution conditions

2. Developing effective separations schemes

3. Determining the behaviors of troublesome components such as iodine and ${ }^{14} \mathrm{C}$

\subsection{Separations for Molten Salt Reactor Fuel Salts}

Research is needed for the capture of fission and corrosion products and certain actinides from molten salts. MSR technology was vigorously pursued in the 1950-1970s at ORNL, culminating in the Molten Salt Reactor Experiment (MSRE) (Haubenreich and Engel 1970). Subsequently, the MSR idea remained relatively dormant until very recently. Resurgence in the MSR field is being driven by several private companies, which are leading the way in new design concepts. Although this has led to substantial advancement of MSR technology, separation challenges associated with maintenance and operation of such reactors has made no significant advancement since the closeout of the MSRE in the 1970s. Realizing the full potential offered by MSRs will require innovative separation technology, especially for restoration and recycle of fuel salts. The inclusion of fast-spectrum MSRs in the future power reactor fleet offers the possibility of fully closing the fuel cycle with the burning of TRU actinides, which would require separation of these elements from a variety of fuels.

High performance for any MSR depends on keeping the neutron parasitic absorptions low. Continuous recycle of the fuel salt means online removal of gaseous, dissolved, and precipitated FPs to mitigate poison loading, corrosion of the reactor containment, and potential embrittlement from constant plating out of radiolytic and/or corrosion products. The ability to continuously perform such separations online is a significant challenge.

Because of the variety of different reactor designs and choice of salts (e.g., fluoride versus chloride), it is difficult to provide a list of specific separation research needs to advance the MSR technology. However, the following principal separation needs can be considered as outstanding challenges.

1. Continuous removal of the neutron poison Xe and other volatile FPs. Continuous He sparging was used throughout the operation of the MSRE to remove the gaseous FPs Xe and Kr. Potentially, ${ }^{3} \mathrm{H}$, 
$\mathrm{HF} /{ }^{3} \mathrm{HF}$, or a combination of both could be released as well. $\mathrm{HF} /{ }^{3} \mathrm{HF}$ are soluble in the fuel salt and are corrosive to the heat exchanger metals and other metals in the reactor core. If the reactor is graphite moderated, these are partially taken up into the graphite and are not easily removed, despite the high fuel-salt temperature. New materials are needed to capture $\mathrm{Xe} / \mathrm{Kr}$ and ${ }^{3} \mathrm{H}$ from the continuous purge stream.

2. Removal of $\mathrm{Pa}$ (applicable to Th-containing molten salt fuels). Advantages of a Th cycle over the U$\mathrm{Pu}$ one include the lower buildup of minor actinides during irradiation and the higher neutron yield per neutron absorbed. ${ }^{233}$ Uranium breeding from ${ }^{232} \mathrm{Th}$ is also feasible with either fast or thermal neutrons. The main difficulty with the technology is that ${ }^{233} \mathrm{~Pa}$ feeds the fissile ${ }^{233} \mathrm{U}$ by $\beta$-decay and can increase potential criticality upon normal or incidental reactor stop events. Consequently, in the breeder mode, the removal of ${ }^{233} \mathrm{~Pa}$ must be continuous and simultaneous with ${ }^{135} \mathrm{Xe}$ removal. During the MSRE, dissolved ${ }^{233} \mathrm{~Pa}$ was removed from the fuel salt by simply mixing it with $\mathrm{ZrO}_{2}$ (Briggs 1966). However, this approach is technologically immature. Developing effective methods for continuous removal of $\mathrm{Pa}$ from molten salt reactor fuels is an important research need that must be addressed.

3. Removal of dissolved FPs. Restoration of MSR fuel salts will require periodic removal of FPs that are dissolved in the salt, such as the lanthanide elements. Methods such as reductive metal extraction were investigated during the MSRE (Ferris et al. 1970), but these methods are not technologically mature and remain undemonstrated. Other applicable separation methods include sorption and crystallization.

4. Separation and recovery of actinides for recycle. Halide volatility or electrochemical methods are the most likely approach for recovery of actinides from MSR fuel salts. The research needs discussed in Chapter 3 are relevant here, but the feed material would be a molten fluoride or chloride salt rather than an oxide-based fuel. Research needs for electrochemical methods are discussed in detail in FRD 7 (Chapter 8). Salt distillation is another possible option. However, the distillation behavior will be different for every salt and its composition. Consequently, specific research directions are difficult to define. Opportunities to explore salt distillation will likely be driven by advances in the development of specific reactors fueled with chloride salts.

\subsection{Summary}

Opportunities exist for improving and simplifying fuel cycle separations through actions taken directly on UNF including molten salts. A diverse set of options should be considered, but most are at relatively low TRL and poorly understood. For oxide-based fuels, voloxidation methods can be applied to produce intermediates such as $\mathrm{UO}_{3}, \mathrm{U}_{3} \mathrm{O}_{8}$, or $\left[\mathrm{UO}_{2}\left(\mathrm{NO}_{3}\right)_{3}(\mathrm{NO})\right]$. A major advantage of voloxidation is the separation of ${ }^{3} \mathrm{H}$ from the fuel matrix so that it can be captured and managed at the front end of the fuel recycling plant (as opposed to distributing throughout the plant). Application of advanced voloxidation techniques could also drive off ${ }^{129} \mathrm{I}$, allowing capture and management of this problematic isotope up front. Skipping the $\mathrm{HNO}_{3}$ dissolution step and dissolving the voloxidized fuel directly in the process solvent offers an intriguing possibility for simplifying fuel recycling flowsheets. Halide volatility methods offer the possibility of eliminating the management of liquid waste streams that is intrinsic to operating a modern PUREX-based reprocessing facility. Although the halide volatility idea has been considered for decades, research is still needed to understand the chemistry and move this concept to industrial application. Supercritical $\mathrm{CO}_{2}$ extraction also offers the possibility of reducing liquid waste volumes generated during processing of irradiated fuel to recover valuable actinide resources. Dissolving the irradiated fuel in carbonate-peroxide media would lead to a new paradigm in fuel recycling. There are broad research needs in all of these areas for processing of irradiated fuel.

MSR fuels offer a completely different set of challenges than oxide-based fuels. Continuous removal of certain neutron poisons (e.g., Xe) is essential to efficient reactor operation. Other FPs and corrosion 
products will need to be periodically removed from the fuel salt to maintain proper reactor operations in terms of the reactor nucleonics and prevention of reactor component corrosion.

\subsection{References}

Anthony, R. G.; Gu, Dosch, D.; Phillip, C. V. Use of Silicotitanates for Removing Cesium and Strontium from Defense Waste. Industrial and Engineering Chemistry Research 1994, 33, 2702-705.

Arm, S. T. Flowsheet Evaluation of Dissolving Used Nuclear Fuel in PUREX Solvent. PNNL-31863. Pacific Northwest National Laboratory, 2021.

Briggs, R. B. Molten-Salt Reactor Program. Progress Report for the Period Ending February 28, 1966. ORNL-3936. Oak Ridge National Laboratory, 1966. https://doi.org/10.2172/4521233.

Brown, H. S.; Seaborg, G. T. Method of Separating Uranium Values, Plutonium Values and Fission Products by Chlorination. US Patent 2875021, February 24, 1959.

Cassayre, L.; Soucek, P.; Mendes, E.; Malmbeck, R.; Nourry, C.; Eloirdi, R.; Glatz, J.-P. Recovery of Actinides from Actinide-Aluminum Alloys by Chlorination: Part I. Journal of Nuclear Materials 2011, 414, 12-18.

Chilenskas, A. A.; Turner, K. S. Engineering Development of a Fluidized-Bed Fluoride Volatility Process. III. Bench-Scale Studies with Irradiated Uranium Alloy Fuels. Nuclear Applications 1968, 4, 6-16.

Del Cul, G. D.; Spencer, B. B.; Hunt, R. D.; Jubin, R. T.; Collins, E. D. Advanced Head-End for the Treatment of Used LWR Fuel. Eleventh Information Exchange Meeting on Actinide and Fission Product Partitioning and Transmutation. 2012, Nuclear Energy Agency, Organisation for Economic Co-Operation and Development, San Francisco, CA.

Enokida, Y.; Sawada, K.; Shimada, T.; Yamamoto, I. An Option Making for Nuclear Fuel Reprocessing by Using Supercritical Carbon Dioxide. 2007, Global 2007, Boise, ID, American Nuclear Society: 1029-32.

Ferris, L. M.; Mailen, J. C.; Lawrance, J. J.; Smith, F. J.; Nogueira, E. D. Equilibrium Distribution of Actinide and Lanthanide Elements Between Molten Fluoride Salts and Liquid Bismuth Solutions. Journal of Inorganic and Nuclear Chemistry 1970, 32, 2019-35.

Gens, T. A.; Jolley, R. L. New Laboratory Developments in the ZIRCEX Process. ORNL-2292. Oak Ridge National Laboratory, 1966. https://doi.org/10.2172/4000618.

Goode, J. H. A Laboratory Study of the Separation and Recovery of Uranium and Plutonium from Fission Products by Chloride Volatility. ORNL-TM-828. Oak Ridge National Laboratory, 1964.

Hariharan, A. V.; Sood, D. D.; Sood, S. P.; Prasad, R.; Sampathkumar, R. Laboratory Investigations in Non-Aqueous Process Chemistry. 1970, Bombay, India, Bhabha Atomic Research Centre.

Haubenreich, P. N; Engel, J. R. Experience with the Molten-Salt Reactor Experiment. Nuclear Applications and Technology 1970, 8(2), 118-36.

Kani, Y.; Sasahara, A.; Hoshino, K.; Kawamura, F. New Reprocessing System for Spent Nuclear Reactor Fuel Using Fluoride Volatility Method. Journal of Fluorine Chemistry 2009, 130, 74-82.

Koegler, S. S. Development of a Unique Process for Recovery of Uranium from Incinerator Ash. Nuclear Energy and the Environment. C. M. Wai and B. J. Mincher. 2010, Washington, DC, American Chemical Society: 65-78. 
Kulyako, Y. M.; Trofimov, T. I.; Samsonov, M. D.; Myasoedov, B. F. Dissolution of Uranium, Neptunium, Plutonium and Americium Oxides in Tri- $n$-Butyl Phosphate Saturated with Nitric Acid. Mendeleev Communications 2003, 13(6), 248-49.

Kurata, M.; Kinoshita, K.; Hijikata, T.; Inoue, T. Conversion of Simulated High-level Liquid Waste to Chloride for Pretreatment of Pyrometallurgical Partitioning Process. Journal of Nuclear Science and Technology 2000, 37, 682-90.

Long, J. T. Engineering for Nuclear Fuel Reprocessing. 1978, La Grange Park, IL, American Nuclear Society.

Mincher, B. J.; Wai, C. M.; Fox, R. V.; Baek, D. L.; Yen, C.; Case, M. E. The Separation of Lanthanides and Actinides in Supercritical Fluid Carbon Dioxide. Journal of Radioanalytical and Nuclear Chemistry 2016, 307(3), 2543-47.

Okabe, P. Gas-Solid Based Chlorination to Support Separation of Actinides and Transition Metals from Rare Earths, Dissertation, University of Utah, 2020.

Okabe, P.; Rappleye, D.; Newton, M.; Simpson, M. F. Development of Metallic Nuclear Material Purification Process via Simultaneous Chlorination and Volatilization. Journal of Nuclear Materials 2021, 543, 152626.

Pitchaiah, K. C.; Lamba, N.; Deepitha, J.; Mohapatra, P. K.; Madras, G.; Sivaraman, N. Experimental Measurements and Correlation of the Solubility of N,N-Dialkylamides in Supercritical Carbon Dioxide. Journal of Supercritical Fluids 2019, 143, 162-70.

Rudisill, T. S.; Shehee, T.C.; Jones, D. H.; Del Cul, G. D. Dissolution of Used Nuclear Fuel using a Tributyl Phosphate/n-Paraffin Solvent. Separation Science and Technology 2019, 54, 1904-11.

Samsonov, M. D.; Shadrin, A. Y.; Shafikov, D. N.; Kulyako, Y. M.; Myasoedov, B. F. Supercritical Fluid Extraction in Modern Radiochemistry. Radiochemistry 2011, 53(2), 111-22.

Scheele, R.; McNamara, B. K.; Casella, A. M.; Kozelisky, A. On the use of Thermal $\mathrm{NF}_{3}$ as the Fluorination and Oxidation Agent in Treatment of used Nuclear Fuels. Journal of Nuclear Materials 2012, 424, 224-36.

Soderquist, C. Z.; Johnsen, A. M.; McNamara, B. K.; Hanson, B. D.; Chenault, J. W.; Carson, K. J.; Peper, S. M. Dissolution of Irradiated Commercial $\mathrm{UO}_{2}$ Fuels in Ammonium Carbonate and Hydrogen Peroxide. Industrial and Engineering Chemistry Research 2011, 50, 1813-18.

Soucek, P.; Cassayre, L.; Eloirdi, R.; Malmbeck, R.; Meier, R.; Nourry, C.; Claux, B.; Glatz, J-P. Recovery of Actinides from Actinide-Aluminum Alloys by Chlorination: Part II. Journal of Nuclear Materials 2014, 447, 38-45.

Stone, J. A.; Johnson, D. R. Measurement of Radioactive Gaseous Effluents from Voloxidation and Dissolution of Spent Nuclear Fuel. 1978, 15th DOE Nuclear Air Cleaning Conference, Boston, MA, US Department of Energy.

Tripp, J.; Soelberg, N.; Wigeland, R. Innovative Separations Technologies. INL/EXT-11-22297. Idaho National Laboratory, 2011.

Uhlir, J.; Marecek, M. Fluoride Volatility Method for Reprocessing of LWR and FR Fuels. Journal of Fluorine Chemistry 2009, 130, 89-93.

Wai, C. M. Reprocessing Spent Nuclear Fuel with Supercritical Carbon Dioxide. In Separations for the Nuclear Fuel Cycle in the 21st Century; G. J. Lumetta, K. L. Nash, S. B. Clark, and J. I. Friese, Eds.; American Chemical Society: Washington, DC; pp 57-67. 
Warren, K. S.; Ferris, L. M. Oxidation and Chlorination of $\mathrm{UO}_{2}-\mathrm{PuO} \mathrm{O}_{2}$. ORNL-3977. Oak Ridge National Laboratory, 1966.

Zhu, L.; Wen, M.; Duan, W.; Xu, J.; Zhu, Y. Application of Supercritical Fluid Extraction in Reprocessing of Spent Nuclear Fuel. Progress in Chemistry 2011, 23(7), 1308-15. 


\section{FRD 3: Design Robust Materials for Separation of Gas-Phase Species}

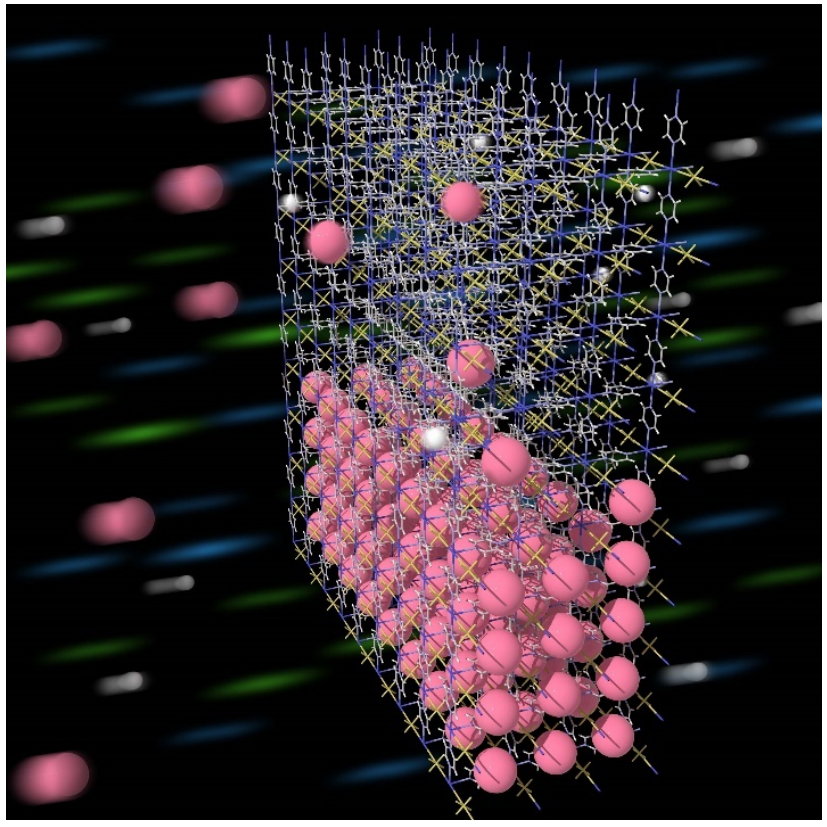

Illustration of noble gas capture by MOF material. (Courtesy of PNNL.)

This FRD prompts consideration of how materials design can be used to advance efficient and cost-effective gas-phase separations and monitoring relevant to the nuclear fuel cycle. Volatile elements or chemical compounds can be released by a wide range of fuel cycle operations, spanning fuel irradiation, fuel processing, and waste stabilization. These volatile materials can be released in many forms and may merit attention based on their value (e.g., ${ }^{3} \mathrm{He}, \mathrm{Xe}$ ), their role in the fuel cycle (e.g., $\left.\mathrm{UF}_{6}\right)$, or their waste management requirements (e.g., compounds containing ${ }^{129} \mathrm{l},{ }^{3} \mathrm{H}$ ). Multiple areas in which advanced materials design could be productively applied include sequestration, separations, sensing, or stabilization of gaseous by-products present during recovery of uranium or other valuable materials. High-priority research needs for advancement of gas-solid separations within the nuclear fuel cycle include (1) accelerating materials discovery, (2) advancing structural forms of novel materials, and (3) applying physical stimuli to materials to effect separations. Integration of separations with disposition is an especially important success criterion.

\subsection{Introduction}

Solid-gas interactions abound within the context of nuclear fuel separations and often occur through the use of solid materials such as sorbents or sieves that partition or stabilize gaseous species. Volatile species are inherent in nuclear fuel reprocessing; any chemical operation that transforms the solid fuel matrix will release volatile elements. With the exception of noble gases, the speciation of the volatile elements released will vary based on the chemistry used. For example, reprocessing techniques such as voloxidation, aqueous dissolution, fluorination, and chlorination result in the release of ${ }^{3} \mathrm{H}$ as $\mathrm{H}_{2}, \mathrm{H}_{2} \mathrm{O}, \mathrm{HF}$, or $\mathrm{HCl}$, respectively. Most commonly, volatile elements or compounds of interest include radioactive noble gases, halogens, ${ }^{3} \mathrm{H}$, and semivolatile transition metals. The volatiles play a special role in reprocessing chemistry, and gas-solid interactions represent an efficient way to effect separations for multiple purposes. 
Broadly, these solid-gas interactions can be classified into three categories: (1) sequestration, (2) separation, and (3) stabilization. Each category is briefly defined below, and the potential utility that robust advanced materials may bring is illustrated. The solid materials that can be used in volatile nuclear fuel cycle separations range from established first-generation technology (such as the use of NaF to concentrate and purify gaseous $\mathrm{UF}_{6}$ ) to specially designed materials intended for a highly specific purpose (such as a silver-phosphate glass precursor designed for iodine capture and subsequent direct vitrification to a stable waste disposal form) (Pénélope et al. 2021).

Sequestration refers to the capture and storage of gaseous species. In nuclear fuel cycle separations this can take many forms but is very commonly employed in effluent treatment with solid sorbent materials being used to capture and store radionuclides that have been volatilized during separation processes. One example of this is the continued research interest from the international community on the sequestration of volatile and radiotoxic iodine isotopes (both ${ }^{129} \mathrm{I}$, which has a half-life of $1.57 \times 10^{7} \mathrm{y}$, and ${ }^{131} \mathrm{I}$, which has a halflife of $8.02 \mathrm{~d}$ ). Highly efficient capture of radioiodine is desired to reduce the potential radioactive dose the public may experience from a nuclear fuel cycle facility by decreasing the gaseous radioactive emissions (Jubin et al. 2012; Bruffey et al. 2020). In seeking to achieve this objective, many specialized iodine sorbents have been designed, manufactured, and tested (Huve et al. 2018). Recent work considered how novel materials design could improve iodine sequestration, but efforts are ongoing to design a robust material that fulfills design requirements. Other notable nuclear applications for sequestration include purification of $\mathrm{UF}_{6}$ through retention on NaF; retention of volatile ${ }^{3} \mathrm{H}$ released during fuel cycle activities; mitigation of $\mathrm{NO}_{x}$ gases arising from traditional fuel dissolution (see FRD 2); and sequestration of $\mathrm{CO}_{2}$ during crushing, burning, and leaching of TRISO fuel (see FRD 1). Major challenges with sequestration of gaseous species arise in that the sequestration materials suffer from low capacity, low selectivity, poor mechanical properties, and susceptibility to chemical and radiolytic degradation.

Separation refers to the ability of a material to remove and collect only specific desired elements or chemical compounds. The need for materials that can affect separations within the nuclear fuel cycle is abundant. One of the highly visible areas requiring advanced materials for separations is noble gas separation. Fuel irradiation and subsequent processing produce a mixture of noble gases (after cooling, primarily comprised of radioactive ${ }^{85} \mathrm{Kr}$ and stable $\mathrm{Xe}$ ). There are many drivers prompting $\mathrm{Kr} / \mathrm{Xe}$ separations research, including reducing the potential off-site dose by removing ${ }^{85} \mathrm{Kr}$ from the plant stack emissions and the desire to recover Xe as a valuable material that can be used for multiple industrial applications. Because of their chemically inert nature, research on noble gas separation has focused on sieving mechanisms that are size based. The primary challenge associated with gas phase separations is achieving selectivity for the desired species.

Stabilization refers the ability of a solid material to provide stable confined storage of a gaseous species. Ideally, this material is specifically designed to enable safe long-term storage and disposition of volatile radioactive gases. Volatile radiological gases pose a special challenge for waste disposal, and there is need for efficient, stable storage materials that can contain high loadings of the target radioisotopes. An ideal material may even perform the sequestration or separations functions needed prior to its use as a disposal media. For example, there are myriad concepts for iodine sequestration from either aqueous or gaseous streams using materials that can be directly converted into stable waste forms for disposal in a deep geologic repository through sintering, melting, or other additive-free processing. Other notable applications for stabilization materials include the stabilization of ${ }^{14} \mathrm{CO}_{2}$, which can be released upon the processing of irradiated graphite, and the stabilization of ${ }^{85} \mathrm{Kr}$, a waste isotope that requires several decades of decay storage before safe release can occur. The primary challenge to stabilizing radioactive gaseous species is designing materials that not only selectively sequester the target gaseous radioisotope but are also sufficiently stable to allow for decay of the captured radioactive isotope. 
The ideal material designed for separation of gaseous species within the nuclear fuel cycle would possess the following attributes:

1. Be highly selective for a single desired species (or a group of target species)

2. Have a high capacity for that species (small footprint)

3. Possess a physical form that is practical for use in common engineering solutions

4. Exhibit high resistance to mechanical, chemical, or radiolytic degradation

5. Serve as a permanent disposition material (unless reuse is desired)

The need for new materials in these diverse applications is clear, but the need for robust materials must be emphasized. Separations within the nuclear fuel cycle must operate under extreme conditions: corrosive, highly radiolytic, and chemically reactive environments. One of the key challenges in achieving effective gas separations in this context is thus identifying materials that will be resilient under these challenging conditions.

\subsection{Technical Challenges in Materials Development for Gas-Solid Nuclear Fuel Cycle Separations}

Several technical challenges face the development of solids that can facilitate separation, sequestration, and stabilization of gaseous species present in nuclear fuel processing. Here, four challenges are described independently, but in several cases these challenges share common solutions and corresponding research thrusts. The four challenges are that (1) identification and testing of materials is time consuming, (2) developing structured materials is difficult, (3) capacities are low and kinetics are slow, and (4) material development should be integrated with final disposition.

\subsubsection{Materials Identification and Testing is Slow}

Historically, solid materials used for separating gaseous species in nuclear fuel cycle applications were identified through trial and error. Many of these materials - intended to be robust within the harsh conditions of the nuclear fuel cycle facilities-were refractory oxides, minerals, metals, or charcoals. In many cases, if chemisorption was desired (e.g., in the case of I

\section{Core Idea}

New approaches to sorption material design are needed to accelerate the rate of gas-phase separation technologies.

immobilized as $\mathrm{AgI}$ ), the reactive species was simply mounted on or incorporated in various porous materials without a fundamentally altering the mechanism of action.

Next generation by-design separation materials and supports are relatively new in nuclear applications. The common sequence of events during development includes (1) identifying and synthesizing the novel material (typically in powder form), (2) testing of the novel sorbent with single gaseous species to generated sorption isotherms and capacity data, (3) optimizing sorption conditions by examining temperature and pressure effects, and (4) continued similar testing with more complex gas mixtures, eventually striving to reach process-relevant conditions. Finally, if the material is judged to merit continued development, it is introduced to more applied research, such as radiolytic testing and development of structured materials (versus the original powdered form). Such a lengthy development pathway is clearly undesirable, especially given the ever-expanding numbers of potential materials to be tested. New, more rapid approaches are needed for discovering and developing separation media for gaseous species. The following paragraphs explore possible future directions. 


\section{Computationally inspired materials discovery}

Within the past decade, materials development has been accelerated through computationally inspired materials discovery. Some materials, including metal-organic frameworks (MOFs), covalent organic frameworks (COFs), and zeolitic imidazole frameworks (ZIFs) have hundreds of thousands of potential structures. Empirically synthesizing and testing the various possible structures would be time and cost prohibitive. However, as described in Sikora et al. (2012), recombination of primary "building blocks" for these structures can be performed quickly via computation to generate thousands of structural permutations. Relevant information, such as pore diameter, cavity diameter, and void volume, can be calculated for each structure to identify the most promising sorbents for synthesis and testing.

Grand canonical Monte Carlo (GCMC) simulations of gas adsorption can then expand upon this screening to (1) predict the best performing materials and (2) elucidate structure-property relationships. For example, GCMC simulations were successfully used to identify CaSDB MOF (also known as SBMOF-1) as harboring the highest reported Xe uptake and selectivity at room temperature under conditions relevant to nuclear fuel reprocessing applications. The CaSDB constructed from sulfonyldibenzoate (SDB) and $\mathrm{CaO}$ clusters, was pinpointed to be highly Xe-selective by molecular simulations in a database containing 5,000 experimental and 125,000 hypothetical MOFs. The computational prediction was then confirmed by conducting laboratory experiments that showed the highest $\mathrm{Xe} / \mathrm{Kr}$ selectivity of 16 in the presence of other gases including $\mathrm{CO}_{2}$ and humidity. Based on the in situ single crystal X-ray diffraction technique, the Xeloaded CaSDB MOF reveals that Xe adsorbs at a single site near the midpoint of the channel, interacting with the channel wall composed of hydrophobic pockets $(4.6 \AA)$ via van der Waals interactions similar to proteins and biomolecules. An excellent description of GCMC adsorption isotherm simulations as applied to noble gas separation is found in Simon et al. 2015.

Although these types of screening and simulating methods are extremely helpful, they have not reached their full potential. The GCMC simulations are often limited to single-component systems (i.e., the adsorption of a gas molecule at varied pressure by a novel material), and they have good accuracy in these cases and in predicting the selectivity of a two-component system by extending the findings of the singleisotherm simulations. However, the targeted gas species is often only an extremely small fraction of the total volume of gas to which a novel material will be exposed. Only after materials synthesis and singlecomponent isotherm validation are completed in an experimental setting (often taking months) does exposure to multicomponent gases begin. In nuclear applications, multicomponent systems may simply contain $\mathrm{O}_{2}, \mathrm{~N}_{2}, \mathrm{H}_{2} \mathrm{O}, \mathrm{CO}_{2}$, and other common air constituents, or they may contain dozens of reactive species. In either case, these other gaseous species may compete with the target species, block the pores of the material, or degrade the novel material. To accelerate materials development, simulation capabilities are needed to predict adsorption properties in the types of complex systems found in nuclear fuel cycle separations. 
Currently, novel porous materials for gas-solid interactions primarily reside in just a few materials classes (MOFs, COFs, ZIFs, etc.). Materials design could be accelerated through a paradigm shift away from the building block/screening process. Biological systems such as proteins exhibit unparalleled efficiency and selectivity in molecular binding and transport while preventing the transport of other chemicals. If the function of biological molecules can be mimicked in synthetic materials, a synthetic host material that selectively transports and binds gases can be designed. As an example, living organisms capture $\mathrm{CO}_{2}$ from both the air and water and convert it into biominerals and fuels under mild, environmentally benign conditions with high efficiency. This capability is enabled by the chemical information contained in proteins, which drives them to fold and assemble into hierarchical structures that carry out complex functions, often by exhibiting conformational changes in response to environmental triggers (Figure 4.1). For example, carbonic anhydrase is a metalloenzyme that accelerates the rate of $\mathrm{CO}_{2}$ hydration to yield bicarbonate

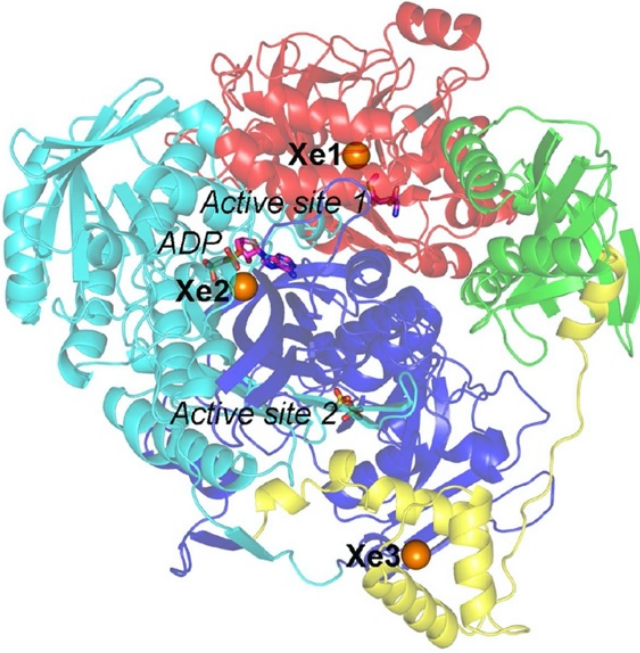

Figure 4.1. Inspiration from biological molecules in binding and transporting noble gases (Tanwar et al. 2019). $\left(\mathrm{HCO}_{3}{ }^{-}\right)$by a rate of up to a million times per second. Along the same lines, RuBisCo enzyme found in many plants, selectively captures $\mathrm{CO}_{2}$ from air using a specific amino acid sequence and converts it into carbamate.

If such effects can be understood and controlled through the use of designed crystalline nanoporous materials, highly efficient and selective separation materials could be realized. A promising strategy is to develop sorbents that mimic biological molecules with desired pore size and shape using the principles of crystal engineering or reticular chemistry. In the case of $\mathrm{CO}_{2}$ released through the processing of irradiated graphite or TRISO fuel, the application of biological design principles may be especially transformative.

\section{Radiolytic degradation}

Like many structured materials, those designed for use in nuclear fuel cycle gas separation applications will be susceptible to radiolytic degradation. Typically, degradation due to these effects is not investigated until many aspects of materials development have been completed, which would already represent a significant investment of development time. There have been only limited attempts to correlate degradation properties with material class. To date, materials tested for degradation include polymers, zeolitic minerals, and few recent reports of MOFs. Many have proven reasonably resistant to induced damage. Research is needed to evaluate a variety of potential materials, binders, and hierarchical structures to provide a broad understanding of what novel material classes are most radiation resistant. Different radiation types $(\alpha, \beta$, and $\gamma$ ) are important in different nuclear fuel cycle applications. 


\subsubsection{Developing Structured Materials is Challenging}

Solid materials intended for use in gaseous separations are often most effective when they are highly porous. Many of these new materials are initially synthesized as fine powders or as low-density, relatively fragile monoliths. Toward industrial implementation of new materials, scaleup necessarily dictates engineering designs such as packed columns, fluidized beds, or other methods that facilitate gas-solid contact. Fragile or powdery material can be undesirable in these types of operations, necessitating research to fabricate engineered forms while maintaining good mass transfer and mechanical stability, often incorporating the material into composites. To find a suitable form for use in cartridges, columns, or membranes may be as challenging as the molecular design of the material itself.

Strategies proposed to overcome this problem involve evaluating various binders and processes to produce differently shaped bodies (pellets, granules, and spheres) through pressing, extrusion, and granulation. In general, shaping of sorbents for gas-solid separations processes improves handling and reduces the pressure drop for flow systems. Several industries produce shaped bodies of various adsorbent materials (e.g., zeolites) for applications in catalysis, separation, and adsorption.

A review of potential methods for structuring porous powders can be found in Ahktar et al. (2014). Hierarchical materials design is critical for advancing the development of novel materials for use in gas-solid nuclear fuel cycle separations, and without it, materials are limited to bench-scale development levels. An illustration of hierarchical materials design is found in Figure 4.2, where materials designed for efficiency at the nanoscale (at the scale of molecular sequestration) are ordered into frameworks of $1 \mathrm{~mm}$ or larger without compromising material properties.

Both structuring processes with and without binders should be advanced with the understanding that in some cases the use of a binder can enhance material durability, whereas in others it may contribute to unreasonably high material volumes after structuring. In-depth laboratory-, bench-, and pilot-scale investigations are essential to

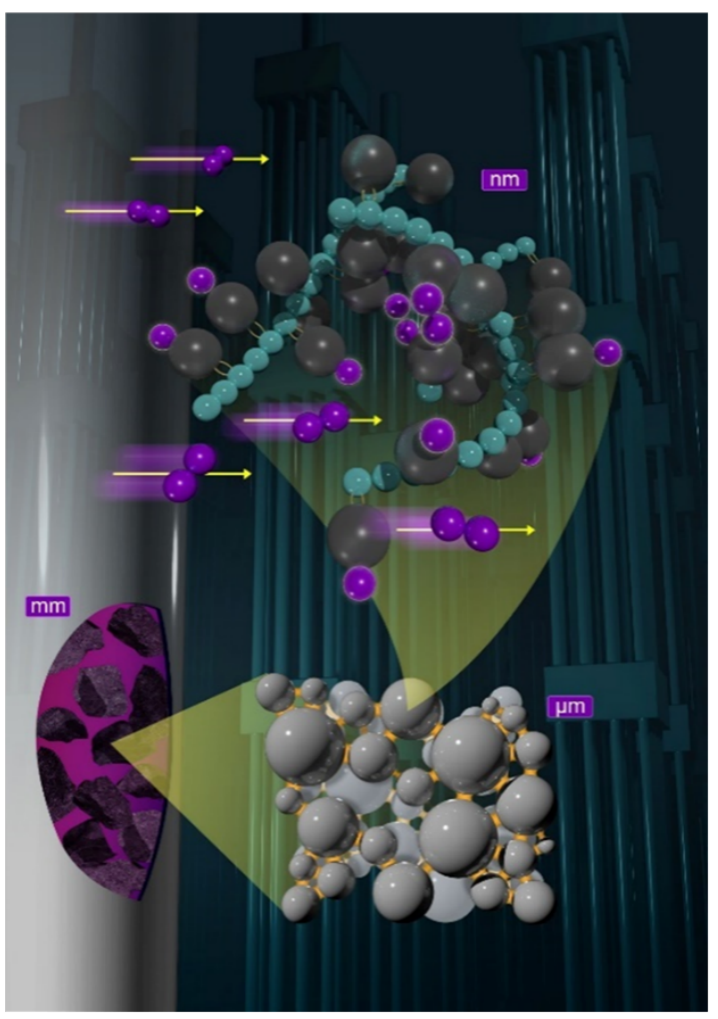

Figure 4.2. Molecular iodine (purple) interacts with a sorbent having hierarchical materials design observable at the nanometer, micrometer, and millimeter scale (Matyáš 2021). improve efficiency and selectivity of sorbents for removal of radionuclides. The focus should be on synthesis and application of multifunctional and sustainable sorbents from natural and engineered materials with high specific surface area, tunable structures, and tailorable surface chemistry (Matyáš 2021). 


\subsubsection{Working Capacity and Slow Adsorption Kinetics are Obstacles to Effectiveness}

Development of new sorbents has relied historically upon chemical phenomena or size-selective sieving, but the effectiveness of these methods can be dampened by materials with low capacities (volume basis) or slow kinetics. However, an opportunity exists for transformational gas separations that are unconstrained by kinetics through reliance on tunable physical properties. These types of induced separations are especially promising for nuclear fuel cycle applications that often require chemically challenging separations (e.g., ${ }^{3} \mathrm{H}$ separations and noble gas separations).

This section provides examples of materials that are controlled through physical means to effect separation

\section{Core Idea}

New strategies to exploit physical stimuli for capture and release of gas-phase species will overcome existing capacity and kinetic limitations. of gases. Utilizing physical properties of materials to tune separative effects of a material is an untapped approach in the field of gaseous separations for nuclear fuel cycle applications. The following presents examples from the field of $\mathrm{CO}_{2}$ capture, but these ideas could be extended to design switchable materials applicable to nuclear related gas separations.

\section{Pressure-responsive materials}

A variety of responsive materials that bind $\mathrm{CO}_{2}$ selectively with subsequent pressure-induced reorganization of molecular building units have been synthesized, suggesting analogous applications for separation of gas-phase species in fuel reprocessing. In one case, a bowl-shaped molecule known as $p$-tertbutylcalix[4]arene (tbc-4) self-assembles into a metastable form depending on the temperature and growth conditions (Thallapally et al. 2008). The bowls stack together to form a bilayer with a pair of tbc-4 groups facing each other to form a skewed capsule. Although there are no inherent channels in the structure, gas adsorption and in situ techniques show that $\mathrm{CO}_{2}$ molecules diffuse through these crystals involving extensive cooperation between $\mathrm{CO}_{2}$ and the tbc- 4 molecules, much like the interactive components of a molecular machine. More specifically, the gas molecules move along the upper rims of the tbc- 4 bowls, where they contact the bulky ${ }^{\mathrm{t}} \mathrm{Bu}$ groups. It is hypothesized that the rotation of these ${ }^{\mathrm{t}} \mathrm{Bu}$ groups would produce "turnstile" effects to make molecular diffusion possible with subsequent reorganization of the metastable molecular building unit.

Light-responsive materials

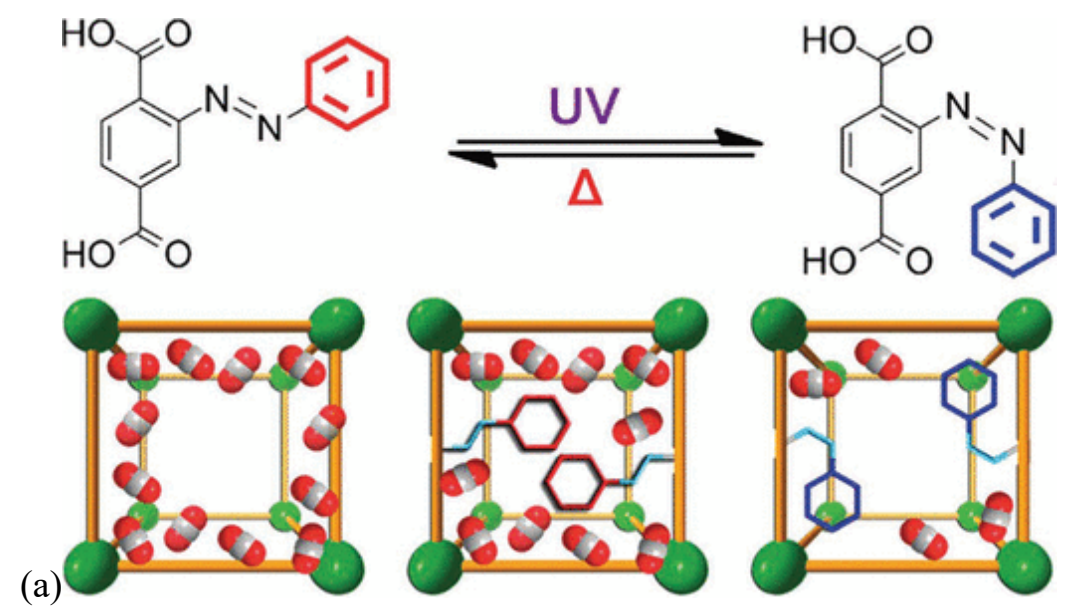


Light stimulus can be used to trigger the wavelengthdependent binding and release of guest molecules in solidstate and solution phases, which has been extensively studied in supramolecular chemistry. For example, various photoresponsive functionalities have been incorporated into porous materials including mesoporous silica, MOFs, and COFs. Among light-responsive MOFs, several strategies were

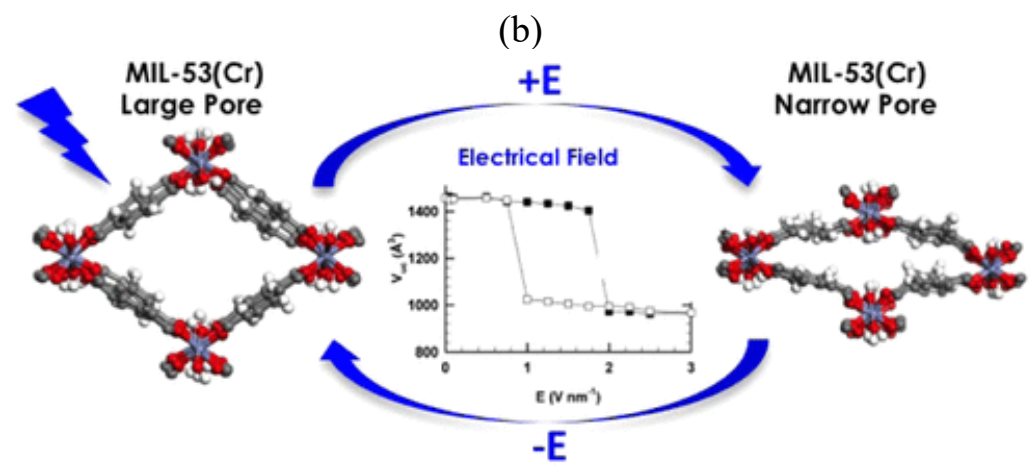

Figure 4.3. Light (a) and electric-field (b) induced structural transformation of molecular solids ([a] Park et al. 2012, reprinted with permission from Journal of the American Chemical Society; [b] Ghoufi et al. 2017). developed that include (1) MOFs constructed from photoresponsive organic linkers (azobenzene derivatives) as a backbone, (2) impregnation of light-responsive groups within the MOF pore, and (3) postsynthetic incorporation of light-responsive molecules into the pore walls of the framework. All of these approaches were developed to control the pore size with subsequent $\mathrm{CO}_{2}$ release upon applied light. For example, a MOF's inner surface was decorated with a light-switchable azobenzene functional group and showed distinct trans/cis isomerization upon UV irradiation. Because of this change in configuration, the total $\mathrm{CO}_{2}$ uptake decreased readily by $26.6 \%$ after UV light irradiation compared with 1 hour, suggesting light-triggered gas release (Figure 4.3) (Park et al. 2012).

\section{Electric field responsive molecular switches}

Like photoresponsive molecular switches, applied electric fields are known to induce molecular changes around the metal center in MOFs or structural transformations in self-assembled organic host molecules as well as polarize molecules to switch the binding affinity. In one study, a flexible MOF bearing terephthalate linkers, known as MIL-53, switches reversibly from a large pore to a narrow pore under an applied electric field (Ghoufi et al. 2017). The dihedral angle between the metal center connected with organic linker undergoes a drastic change upon application of an electric field with subsequent reorganization of the MOF. When using the narrow-pore form under the applied electric field, the MOF loses its flexibility and becomes more rigid. This narrow-pore form can then be used to separate gases based on a size-exclusion principle otherwise not possible because of its flexible nature. This phenomenon is not unique to MIL-53 and can be considered a general approach for responsive sorbent materials that could be used for fuel cycle separations.

There are other mechanisms beyond crystal transformations in which electric fields can induce polarizations of a material to generate improved bonding affinity. In one study of $\mathrm{MoS}_{2}$, molecular dynamics simulations were used to see how an electric field impacted the adsorption of $\mathrm{CO}_{2}$ molecules (Sun et al. 2017). During this study, it was found that at a specific electric field, the mechanism of $\mathrm{CO}_{2}$ binding on $\mathrm{MoS}_{2}$ changes from physisorption to chemisorption. The change in mechanism was due to the polarization of the Mo-S bond that led to charge transfer from the Mo atom to the $\mathrm{S}$ atom. This transfer created a better bonding site for $\mathrm{CO}_{2}$ and led to the change to a chemisorption mechanism. 


\subsubsection{Materials Development should Consider Final Disposition}

\section{Core Idea}

Revolutionary improvements in system performance may be gained by designing separation materials that can be readily converted to immobilized waste forms for final disposition.
Most materials are not developed with an eye toward eventual disposition as radiological waste. This conflicts with the concept of an integrated nuclear fuel cycle in which valuable resource utilization is maximized and radiological waste generation is minimized. Nuclear fuel cycle separations cannot be performed independent of waste management; the two are integrally connected. This principle applies to gaseous separations just as it does to other aspects of separations.

With this fundamental principle in mind, the highest priority should be placed on the developing materials that actually facilitate disposal of the radioactive FP of concern. Several of the research thrusts in this document align with this priority. An integrated novel materials-development strategy should consider the fuel component being separated and should tailor the waste disposal needs of that component. As examples, abundant research exists on the direct consolidation of zeolite and aerogel iodine sorbents into densified final waste forms. Increasing the capacity of noble gas sorbents aligns with the need to decay-store ${ }^{85} \mathrm{Kr}$ for several decades in reasonable volumes of storage space. The use of physically tunable and reversible separations may facilitate the generation of a customized waste stream that can be easily converted into a durable waste material. The development of structured adsorbents may allow for simplified densification or consolidation of waste materials. A guiding principle for materials development for gas-phase separations should be the integration of eventual disposition into material design.

\subsection{Summary}

In summary, several high-priority research thrusts promise advancement of gas-solid separations within the nuclear fuel cycle:

1. Accelerate material discovery. Materials discovery must be accelerated through new computational methods, paradigm-shifting design inspiration, and a more systematic understanding of radiolytic degradation across material classes.

2. Engineer materials for process application. The structuring of these novel materials is critically important, especially in the harsh environments associated with nuclear separations. Approaches to maximize porosity and capacity and increase the mechanical strength of the materials must be identified as enhanced by hierarchical materials design.

3. Exploit physical property switching for separation efficiency. Gas-solid chemistry within the nuclear fuel cycle often deals with species that are challenging to concentrate, but high affinity makes release for downstream processing and reuse of the sorbent material difficult. For this reason, the development of physical property-induced binding and release can be transformative.

4. Integrate development of separation material with form development. Each of these research thrusts is incomplete if the ultimate waste disposition form is not considered. Projects that demonstrate integration with separations and disposition must be prioritized.

Pursuit of these research thrusts is very promising for developing an ideal, efficient, and cost-effective gassolid separation approach for nuclear fuel cycle applications. 


\subsection{References}

Akhtar, F.; Andersson, L.; Ogunwumi, S.; Hedin, N.; Bergström, L. Structuring Adsorbents and Catalysts by Processing of Porous Powders. Journal of the European Ceramic Society 2014, 34(7), 164366.

Bruffey, S.; Soelberg, N.; Martin, L. R.; Peruski, K. Requirements and Conceptual Design of Off-Gas Systems for the Reprocessing of Metallic Fuels. ORNL/TM-2020/1668. Oak Ridge National Laboratory, 2020.

Ghoufi, A.; Benhamed, K.; Boukli-Hacene, L.; Maurin, G., Electrically Induced Breathing of the MIL53(Cr) Metal-Organic Framework. ACS Central Science, 2017, 3, 394-98.

Huve, J.; Ryzhikov, A.; Nouali, H.; Lalia, V.; Augé, G.; Daou, T. J. Porous Sorbents for the Capture of Radioactive Iodine Compounds: A Review. RSC Advances 2018, 8(51), 29248-73.

Jubin, R. T.; Soelberg, N. R.; Strachan, D. M.; Ilas, G. Fuel Age Impacts on Gaseous Fission Product Capture during Separations. PNNL-22550. Pacific Northwest National Laboratory, 2012.

Matyáš, J. Advancements in Sorption Technologies for Nuclear Applications. Presented at the Innovative Separations R\&D Needs for Advanced Fuel Cycles Workshop, Oak Ridge, TN, August 2021.

Park, J.; Yuan, D.; Pham, K. T. Reversible Alteration of $\mathrm{CO}_{2}$ Adsorption upon Photochemical or Thermal Treatment in a Metal-Organic Framework. Journal of the American Chemical Society 2012, 134, 99-102.

Pénélope, R. ; Campayo, L. ; Fournier, M. ; Gossard, A. ; Grandjean, A. Silver-Phosphate Glass Matrix for Iodine Conditioning: From Sorbent Design to Vitrification. Journal of Nuclear Materials 2022, 558, 153352.

Sikora, B. J.; Wilmer, C. E.; Greenfield, M. L.; Snurr, R. Q. Thermodynamic Analysis of Xe/Kr Selectivity in over 137000 Hypothetical Metal-Organic Frameworks. Chemical Science 2012, $3(7), 2217-23$.

Sun, Q.; Qin, G. Q.; Ma, Y. Y.; Wang, W. H.; Li, P.; Du, A. J.; Li, Z. Electric field-controlled $\mathrm{CO}_{2}$ capture and $\mathrm{CO}_{2} / \mathrm{N}_{2}$ separation on $\mathrm{MoS}_{2}$ monolayers. Nanoscale 2017, 9(1), 19-24.

Tanwar, A. S.; Goyal, V. D.; Choudhary, D.; Panjikar, S.; Anand, R. Importance of Hydrophobic Cavities in Allosteric Regulation of Formylglycinamide Synthetase: Insight from Xenon Trapping and Statistical Coupling Analysis. PLos One 2013, 8, e77781.

Thallapally, P. K.; McGrail, B. P.; Dalgarno, S. J.; Schaef, H. T.; Tian, J.; Atwood, J. L. Gas-Induced Transformation and Expansion of a Non-Porous Organic Solid. Nature Chemistry 2008, 7(2), $146-50$. 


\section{FRD 4: Apply Physical Phenomena, Fields, and Gradients to Intensify Separations}

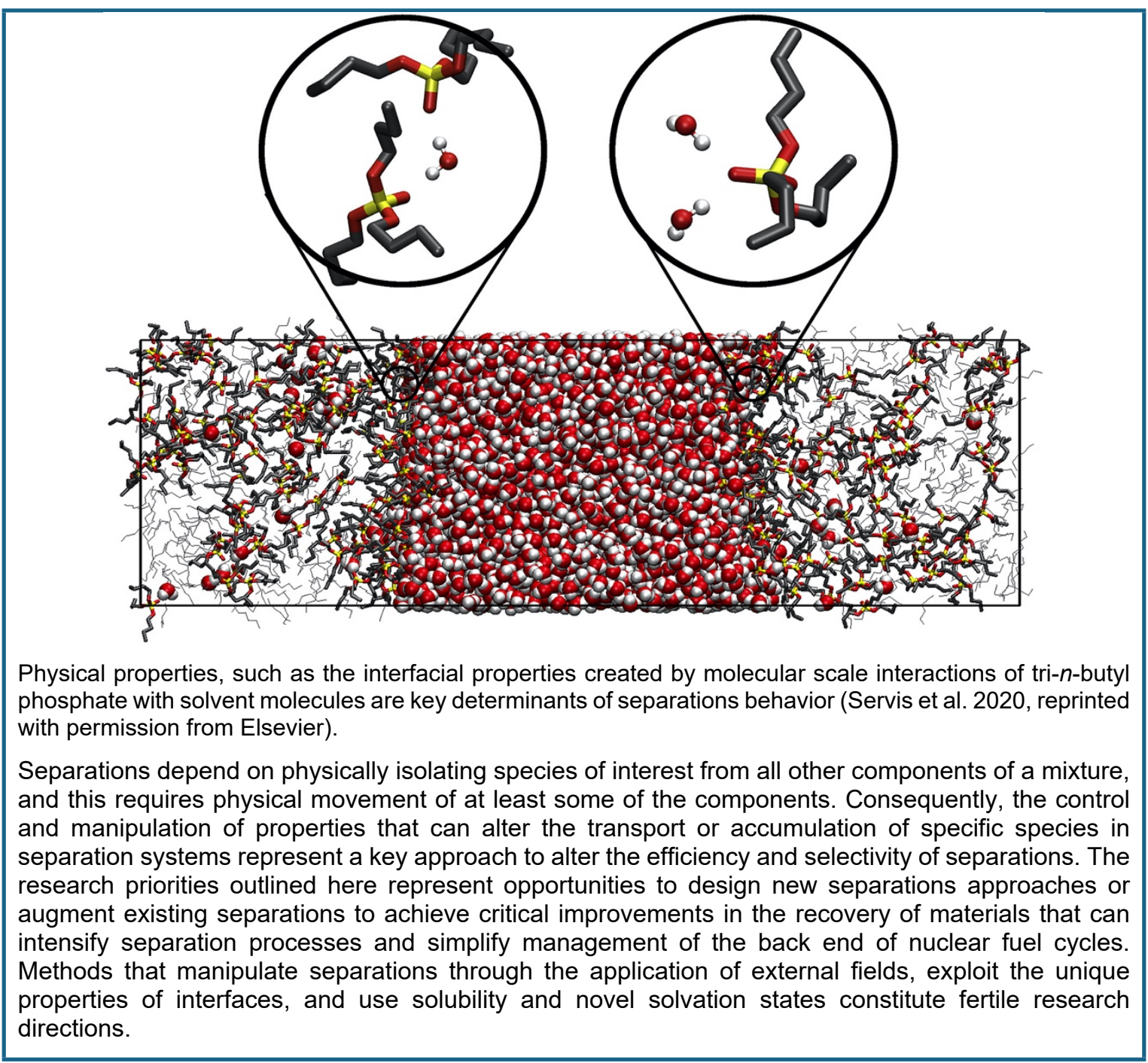

\subsection{Introduction}

The baseline separation processes for UNF have been practiced for decades. The hydrometallurgical PUREX solvent extraction process, which is used around the world to recover $\mathrm{U}$ and $\mathrm{Pu}$, was first proposed in the 1940s (Lantham and Runion 1949), and electrorefining of sodium-bonded fuel has been conducted at Idaho National Laboratory (INL) for more than 25 years (Li et al. 2005). Though successful, these processes are hampered by their own limitations. At the most fundamental level, the goal of all the separations suggested or described in this workshop report is the spatial separation of particular components of nuclear fuel. Most separations accomplish this by the transfer of the components between different physical phases, whether between two liquid phases in solvent extraction, between condensed phases and the gas phase in separations such as voloxidation or selective off-gas capture, or between liquid and solid phases in electrodissolution/electrodeposition, chromatography, or precipitation. Phase transfer is not, however, an essential component of separations, and some important separations take place in a single phase. For example, isotopic separations of $\mathrm{U}_{\text {as }} \mathrm{UF}_{6}$ take place solely in the gas phase, various ion mobility 
techniques (such as electromigration) separate species solely in the gas or liquid phases, and field-flow fractionation exploits the effects of a variety of external fields to separate the constituents of liquid phase samples.

Despite the central role of the actual spatial separation of components, developments in fuel cycle separations have primarily considered approaches that directly increase the specificity of the separation for the targeted components, such as by complexation or manipulation of oxidation states instead of approaches that alter the properties of the overall system to affect the movement and partitioning of the target species. Thus, exploiting physical phenomena to create better separations is an area where critical advances in separations of irradiated nuclear fuel could likely be realized. Development of intensified separation processes that are more economical with smaller footprints, less energy consumption, lower reagent use, higher throughput, and reduced waste generation is the overarching goal of this future research direction.

As shown in the following examples, ongoing advances in separation science and engineering provide a framework to intensify nuclear separations through physical phenomena:

- Centrifugal contactors combine reaction, mass transfer, and phase separation into a single unit, reducing the footprint and increasing the throughput of liquid-liquid or liquid-gas separations by creating high centrifugal forces. However, they require rapid partitioning kinetics to fully realize the full throughput possible (Brown, Wardle et al. 2016).

- Membrane separations driven by concentration gradients offer simplicity and small material inventories, although fouling, membrane stability, and throughput can be issues (de Gyves and Rodríguez de San Miguel 1999).

- Unprecedented detail in computational and experimental studies of interfacial species is providing new mechanistic insight into material transport at interfaces (Chowdhury et al. 2020; Lovering et al. 2020; Liu and Clark 2021) and are opening avenues to consider novel pathways to intensify separations of nuclear fuel by directly tuning the interfacial properties.

Such advances raise the question: Can advances in applying external fields and gradients, interfacial chemistry, or physical properties to separations be exploited to radically improve separations of nuclear fuel and simplify waste disposal?

\subsection{Harnessing External Forces and Fields for Separations}

The standard approaches to hydro- and pyrometallurgical separations of UNF rely on the formation of dissolved ions or molecules that can be transported to another region or zone in the process where they can be recovered through the application of particular stimuli within the solution that change the nature or activity of chemical species present. The

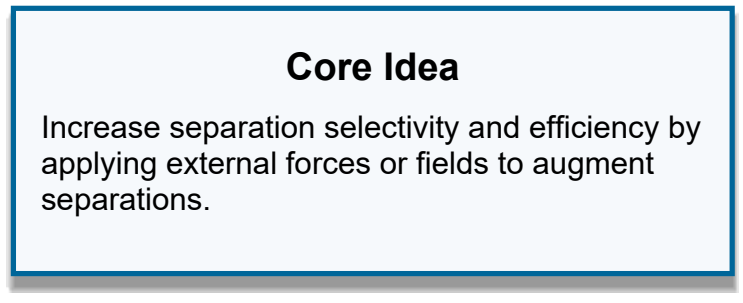
following list provides common examples of such approaches, which span a wide range of systems:

1. Altering the electrochemical potential $\left(E_{h}\right)$ to cause reduction or oxidation and alter the chemical properties of the target species:

a. Anodic dissolution and cathodic deposition of actinides in molten salt solutions

b. Plutonium partitioning in the PUREX process through formation of $\mathrm{Pu}(\mathrm{III})$ by reduction with chemical reductants such as Fe(II), U(IV), or hydrazine 
2. Shifting the equilibria controlling the complexes present through changes in acidity or counterion concentrations:

a. Uranium/thorium separation in the THOREX (thorium extraction) process

b. Purification of tetravalent $\mathrm{Np}$ or Pu by anion exchange from $\mathrm{HNO}_{3}$ media

3. Introducing reagents that produce new species with distinct properties:

a. FP removal from molten salts by addition of zeolites

b. Americium and curium separation from FP lanthanides by solvent extraction in the reverse TALSPEAK (trivalent actinide-lanthanide separation by phosphorus reagent extraction from aqueous komplexes), i-SANEX (innovative selective actinide extraction), or ALSEP processes

These established approaches chemically transform the fuel components to achieve separation. The shortcomings of these approaches and opportunities to drive vital research in those areas are described in other parts of this report. However, a variety of external forces or fields can influence the physical separation of species without chemical transformations, and these forces warrant investigation to intensify nuclear separations through unique mechanisms of action.

\section{Electrical fields}

When dissolved in solution, the major components of UNF exist as ions, and the unique electrical properties of specific ions - particularly their effective charge density — can be exploited for separations. Electrical field-enhanced separations are presently pursed in a variety of large-scale applications that cut across a range of separation methods including solid-solid separations in the beneficiation of ores, enhanced phase separation in liquid-liquid separations, and solid-gas separations. In addition, electrically driven separation of ions by electrophoresis or through phase transfer at liquid-liquid or solid-liquid interfaces are well known processes that can be used to determine a range of species in UNF (Stockmann and Ding 2011; Willberger et al. 2019). Although advances in these areas of analysis could be employed in real-time monitoring and analysis of separation processes considered in FRD 6, the application of electrical fields in separations of nuclear fuel could surmount key issues in existing separation processes, such as Tc control, by altering the physical transport of specific ions. Specific examples of industrial-scale separations driven by electrical fields include electrodialysis for water desalination or recovery of carboxylic acid from the synthesis milieu. Such electromembrane separation processes employ selective solid or liquid membranes that bring together chemical selectivity for particular species and field-driven ion transport across an interface, and the applied electrical potential provides the principal thermodynamic driving force for the separation. When an electrical field drives the separation, reagent consumption and associated generation of secondary wastes can be minimized.

\section{Magnetic fields}

Magnetic separations of paramagnetic species as particles, magnetic fluids, or even in living cells are well known, but recent work suggests that magnetic fields can influence the separation of discrete ions in dynamic separations processes as well. For example, selective separation of the paramagnetic lanthanide dysprosium from a mixture containing diamagnetic La(III) and Dy(III) by recrystallization was enhanced by a factor of 2 in the presence of a magnetic field (Higgins et al. 2020). 
The magnetic susceptibilities of the components of used fuel vary widely (Figure 5.1). The ions of light actinide elements Th through Am possess small susceptibilities that vary from element to element and also depend on the oxidation state of the same element. In contrast, the heavy actinides $\mathrm{Cm}$ (III) and $\mathrm{Cf}(\mathrm{III})$ display large magnetic susceptibilities that could potentially enable facile magnetically assisted separation of $\mathrm{Cm}$ and $\mathrm{Cf}$ from $\mathrm{Am}$ as well as magnetic enhancement of Am(III)Ln(III) separations in high-

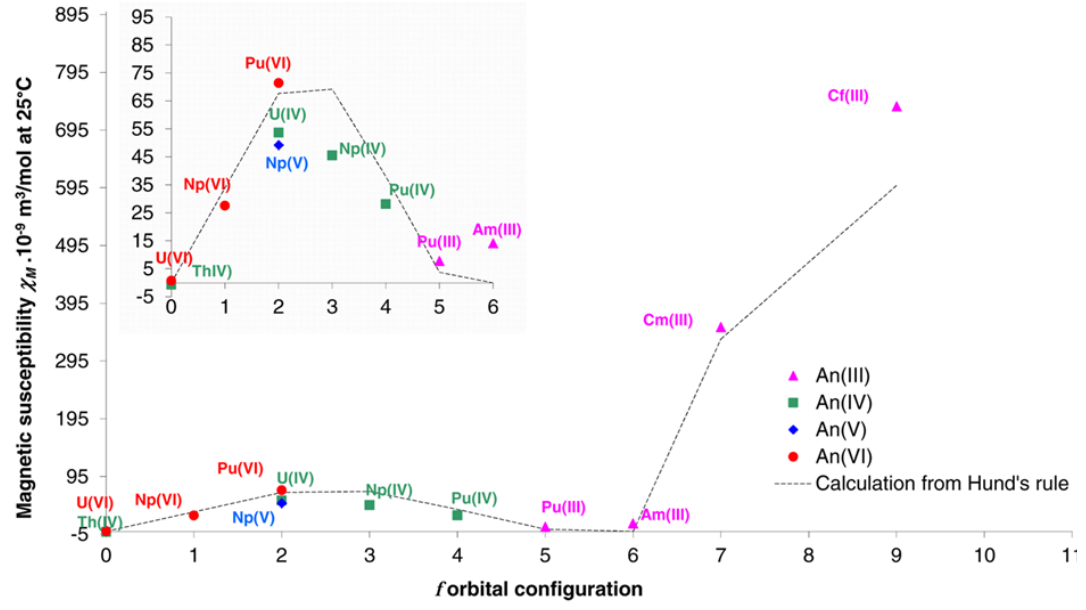

Figure 5.1. The magnetic susceptibilities of actinide ions suggest the possibility of magnetic separation of ionic species in irradiated fuel (Wall et al. 2014, reprinted with permission from Journal of the American Chemical Society).

burnup advanced reactor fuels, which would serve to reduce the neutron hazard and heat generation when fabricating Am-containing fuels. Such magnetic separation technology could enhance separations in a variety of hydrometallurgical and pyrometallurgical separations, favoring the transport of target elements or retarding the transport of impurities.

\section{Light}

Light-driven separation of ions relevant to nuclear fuel recycle have been approached from several angles. These approaches offer unique pathways to generate selectivity for individual species when the intrinsic chemistry of a target species is not sufficiently distinct to allow conventional chemical separations. Examples of these diverse approaches include selective photooxidation or photoreduction of metal ions or ligands (Donohue 1979; Hirai and Komasawa 1995; Salpage et al. 2018), photodissociation or photosubstitution of metal complexes (Donohue 1978), or photoswitchable ligands where light triggers ion complexation or release (Malval et al. 2002; Hua and Flood 2010). Particular strengths of light-driven separations lie in the selectivity that can be achieved by using a particular wavelength of light and the irreversibility of properly designed light-initiated processes, while drawbacks include the possible presence of other species that absorb at the wavelengths of light necessary to trigger the desired transformation of the target species. Although some nuclear processing solutions (such as dissolved fuel) will be too opaque to be amenable to light-driven separations, downstream separations of key species appear feasible.

\section{Other fields}

Beyond electrical-, magnetic-, and photo-enhanced separation, other fields should also be considered for intensifying fuel cycle separations. For instance, the creation of artificial gravitational fields through high centrifugal forces can improve separation performance substantially, and separations of $\mathrm{U}, \mathrm{Pu}$, and $\mathrm{Am}$ from MOX fuel (Litvina et al. 2007) and separations of Am and $\mathrm{Cm}$ have been demonstrated in planetary centrifuges (Maryutina et al. 2004). Another potentially relevant approach to improve separations of nuclear fuel is the application of thermal gradients to drive separations, which are already used in gas-phase separations and in liquid-based separations such as thermal-gradient liquid chromatography and thermal field-flow fractionation. 
Industrial experience with process intensification and the limited investigations using external forces or fields to separate elements encountered in used fuel suggest that such augmentation of conventional hydrometallurgical or pyrometallurgical separations can boost selectivity for particular species. However, the application of external forces or fields to fuel cycle separations remains largely unstudied. The following key areas of research are necessary to advance field-enhanced separations of nuclear fuel:

1. Develop novel field-enhanced separation methods. The state-of-the-art in hydrometallurgical and pyrometallurgical separations is well defined and the intrinsic thermodynamic and kinetic limitations of current separation systems are understood, but the degree of enhancement and the limitations of field-enhanced separations for used fuel are unknown. Research focused on enhancement of specific critical separation needs, such as Tc or $\mathrm{Np}$ rejection or actinide/lanthanide separations, with fields and the design of flowsheets and process equipment for novel separations that incorporate external fields is essential.

2. Develop new materials to enable field-enhanced separations. New materials will be essential to enable field-enhanced separation. Electrically enhanced liquid-liquid separations will require novel conductive solvents, such as hydrophobic ionic liquids, that can support the currents necessary for significant ion transfer between phases. In synergy with FRD 5, materials for ion-specific membranes, either solid or liquid, that incorporate particular binding groups can provide the baseline separation that electrical or magnetic fields enhance, whereas the deployment of light-driven separations will require new ligands that can serve as antennas for light absorption or as an actual photoswitch with binding properties that are modulated by specific frequencies of light.

3. Characterize radiation effects on field-enhanced separation systems. The effects of radiation fields on field-enhanced separations are largely unknown. The large and constant background of radical species generated by the interactions of the $\alpha, \beta$, and $\gamma$ radiation emitted by the fuel constituents are a challenge to many separation systems. Their effects could range from interference in magnetically enhanced separations to degradation of membranes or complex ligands. Development of deployable fieldenhanced separation systems must consider such effects.

\subsection{Controlling Interfacial Chemistry to Drive Separations}

Phase-transfer reactions between two liquid phases, as in solvent extraction or liquid membrane separations, or between a liquid phase and a solid phase, as practiced in ion exchange chromatography or electrodissolution and electrodeposition are vital components of many separations. The kinetics of separation processes are often determined by interfacial processes. The interfacial area available for interphase transport is

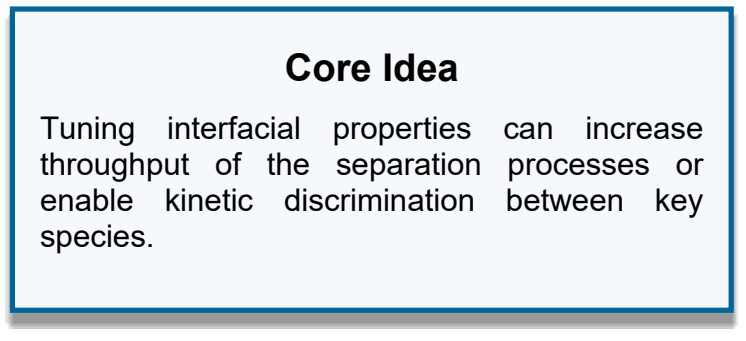
inherently limited, and ligand-exchange reactions of the most important species in UNF (Cs, Sr, lanthanides, and actinides) in the bulk phases are relatively fast. Consequentially, the rates of many liquidliquid and liquid-solid separations are determined by processes in the interfacial region rather than chemical reactions occurring in the bulk aqueous or organic phase (Danesi 1992), and control of the properties of the interface between these phases represents another important area where separations could be intensified.

Most separations of nuclear fuel strive to operate in or near thermodynamically controlled regimes where interfacial intermediates are not directly related to the degree of separation. However, full control of separations founded on interphase transport requires more than control of bulk-phase equilibrium 
properties. The reactions at the interface often determine the overall rate of the separation, even if they do not affect the ultimate separation selectivity, and the physical properties of the interface exert strong influence on attributes that are critical to highly efficient separations such as surface wettability, drop size, and drop formation and coalescence. However, the details of the molecular-level interactions at the interface are difficult to probe experimentally and, particularly in hydrometallurgical separations, they are only beginning to be discerned.

Control of interfacial separations processes can open many avenues to intensify nuclear separations, but a much deeper understanding of the molecular-level structure and behavior of the interfaces of separation systems is necessary to gain the requisite control of mass transport at the microscopic level. It must be recognized that the interfacial region is not well represented by a simple plane at the point of contact between two phases. Instead, it is a zone of progressively changing chemical composition that can be highly ordered or can display distinct, dynamic molecular-level heterogeneity during separation processes (Servis and Clark 2019). The ions and molecules responsible for the interfacial behaviors and mass transport rates of key species are not well known, but they may not resemble the species known to exist in the bulk phases of nuclear separation systems. Conclusive identification of these species coupled with an understanding of how solvent properties affect the species formed and the mechanisms of mass transport are critical areas for development.

Certain species may also be confined to the interface, experiencing or inducing a variety of confinementrelated effects. Other confounding factors involve the behavior and action of other solutes during the separation. Because the interfacial region represents only a small fraction of the total volume of most the separation systems, highly surface-active solutes can affect the interface even at very low bulk-phase concentrations. In addition, fouling of the interface by species unrelated to the phase transport can completely shut down the phase transfer processes. These complexities of interfacial chemistry each require attention to achieve predictive control of interfacial chemistry and processes, but they will be insufficient in themselves to achieve the most efficient separations possible. Molecular-level insights into interfacial behavior also will need to be combined with advances in understanding the chemical properties of relevant metal ions and their interactions with ligands to design high intensity separation systems that avoid unnecessary kinetic bottlenecks.

\section{Research Needs to Intensify Separations through Interfacial Chemistry}

Although interfacial processes are critical to separations of nuclear fuel, very few molecular-level details of these processes in nuclear energy systems are well understood. The present state of knowledge of these systems suggests that more efficient separations are possible, but the many knowledge gaps leave substantial uncertainty in how to exploit interfacial processes and properties for process intensification. The following key areas require research to exploit the potential of interfacial processes to intensify nuclear separations:

1. Understand the molecular-level factors controlling the rate of interfacial processes. Effective structure-property relationships and studies of the interfacial chemical species present in relevant solvent extraction, ion exchange, and certain electrometallurgical separations are needed to define and predict the kinetics of separations processes in order to develop intensified separations that operate at the diffusion limit even for the fastest separations.

2. Control interfacial phenomena to enable kinetically controlled separations. The most important species encountered in separations of nuclear fuel generally display fast reaction kinetics. Nevertheless, systematic deviations in the separation kinetics are known for some species that display little thermodynamic separation, such as Am(III) and $\mathrm{Cm}$ (III). Robust kinetic separations of important constituents of UNF would augment separations based on thermodynamic selectivity. Novel strategies 
using interfacial chemistry to control the kinetics of separations could include harnessing interfacial microstructures in liquid-liquid extractions to tune the extraction rate of particular species, development of ion exchange materials with rapid kinetics that could be coupled to field-based separations, or interfacially controlled electrokinetic separations.

3. Characterize interfaces and interfacial species at a molecular level. Separation systems directly relevant to nuclear separations need to be studied using the latest generation of interfacially sensitive experimental techniques, with systems building up from simplified systems containing a minimal number of components at optimal concentrations to systems that reflect a substantial degree of the complexity of real separation systems, including the presence of radioactive elements, degradation products, and other potentially surface-active constituents.

4. Develop and experimentally validate theoretical models. Computational studies have emerged as key tools to explore the specific processes that occur at interfaces and to compare and contrast interfacial reactions with those that occur in bulk phases. However, the difficulty of conducting experiments on interfacial species and processes leaves few experimental results that can be directly connected to computational studies. A variety of validating separation systems, germane to nuclear fuel separations, that span relevant pyro- and hydrometallurgical separations need to be developed to ensure the validity of computational studies of separations interfaces and interfacial processes.

5. Understand and control interfacial fouling. The performance of separation systems often degrades over time owing to the buildup of interfacially active impurities and degradation products. This leads to issues such as slow phase disengagement and the formation of interfacial emulsions or CRUDs in solvent extraction and the blinding of membranes or ion exchange materials. These processes will hamper efforts to intensify separations. The tools and approaches developed and employed in the preceding research needs for interface science and engineering should focus on understanding the underlying causes of fouling and bringing about science-based remedies.

\subsection{Solubility Controlled Separations}

Precipitation and crystallization are time-tested separation techniques that formed the basis for the first macroscopic purification of $\mathrm{Pu}$ through the bismuth phosphate process. Precipitation continues to find applications in nuclear separations on the front end of the nuclear fuel cycle, producing the $\mathrm{U}$ compounds

\section{Core Idea}

Simplify separations by incorporating selective, high-efficiency precipitation or crystallization.

collectively known as yellowcake from purified ores, and on the back end of the fuel cycle where plutonium oxalate is often precipitated as an intermediate in the production of $\mathrm{PuO}_{2}$. Although useful for isolating solid materials, precipitative separations have been largely supplanted by other techniques because precipitation is perceived to display low selectivity for similar ions, to be difficult to implement as a continuous process or with repeated dissolution-precipitation cycles, to generate voluminous wastes that are difficult to dispose, and to present unique criticality concerns. However, selective precipitation of species with distinct chemical properties can simplify separation flowsheets, particularly if it is paired with selective dissolution such as the alkaline-side processing proposed in FRD 2 or the sorts of ligand-design concepts considered in FRD 5.

Under the radiation fields encountered in the separations of irradiated fuel, separations using simple inorganic precipitating agents are desirable. Tri- and tetravalent actinides form insoluble compounds with many simple anions, such as $\mathrm{F}^{-}, \mathrm{PO}_{4}^{3-}, \mathrm{IO}_{3}^{-}, \mathrm{O}_{2}{ }^{2-}, \mathrm{C}_{2} \mathrm{O}_{4}{ }^{2-}, \mathrm{CO}_{3}{ }^{2-}$, or $\mathrm{OH}-$; these are not, however, particularly selective precipitants. On the other hand, actinides in higher oxidation states, the actinyl(V) and actinyl(VI) ions, have chemistries the differ from all the other elements in the periodic table. They tend to be more soluble than are actinide(III) and actinide(IV) ions, forming soluble species with $\mathrm{F}^{-}, \mathrm{IO}_{3}{ }^{-}, \mathrm{O}_{2}{ }^{2-}, \mathrm{CO}_{3}{ }^{2-}$, and 
even $\mathrm{OH}^{-}$under particular conditions. The one-of-a-kind solubility pattern of the actinyl(VI) cations $\mathrm{UO}_{2}{ }^{2+}$, $\mathrm{NpO}_{2}{ }^{2+}, \mathrm{PuO}_{2}{ }^{2+}$, and $\mathrm{AmO}_{2}{ }^{2+}$ coupled with the high concentration of these elements in UNF represents an opportunity to combine the specificity of a precipitative separation for unique cations (e.g., $\mathrm{UO}_{2}{ }^{2+}$ ) with poor selectivity for similar species (i.e., $\mathrm{NpO}_{2}{ }^{2+}, \mathrm{PuO}_{2}{ }^{2+}$, and $\mathrm{AmO}_{2}{ }^{2+}$ ) to conduct proliferation-resistant group separations of the light actinides from used fuel. Such an approach to co-crystallize these four actinyl(VI) cations from the solutions presently obtained by dissolving UNF in nitric acid has been proposed (Burns and Moyer 2016), requiring a preliminary strong oxidation step to convert Am(III) to Am(VI). The subsequent co-crystallization of the hexavalent actinides repurposes the chemistry of the NEXT (new extraction system for TRU recovery) process, which had originally been designed to simplify subsequent liquid-liquid separations by initially removing the majority of the U sans TRUs in dissolved fuel as crystalline uranyl nitrate hexahydrate (Chikazawa et al. 2008).

Crystal engineering has the potential to take the next step beyond selective precipitation of simple salts to target species for selective crystallization. Here, a crystallization agent added to the solution enables the formation of a crystalline lattice that incorporates the ions targeted for separation from the mixture. The agent can be either organic or inorganic, interacting with the target ion and promoting extended interactions across a lattice. Ideally, the structure of the crystal has the additional function of excluding other solution components because of the structure and rigidity of the framework, which amplifies the selectivity. The function of the crystallization agent can be to directly linked the target ion as nodes in the lattice in the manner of coordination polymers (Mukherjee and Zaworotko 2020) or to encapsulate simple or complex ions wherein the complex will engage in secondary interactions with the extended lattice. Such approaches are being successfully applied, for example, in carbonate precipitation for direct air capture of $\mathrm{CO}_{2}$ (Custelcean 2021).

\section{Research needs for solubility-controlled separations}

Strategies to exploit the intrinsic solubility of inorganic species, like those embodied in the NEXT process, could greatly simplify or even supplant current hydrometallurgical separation processes. Research is required in the following key areas to realize the potential of precipitative separations to streamline nuclear separations:

1. Couple selective dissolution and crystallization. The simpler the feed into a crystallization process is, the less likely it will have contaminants in the crystallized material that needs to be removed by washing. Overarching strategies to combine selective dissolution of used fuel with efficient group separations of light actinide elements are needed to boost overall selectivity of such processes. Furthermore, crystal engineering approaches that effectively target essential species after selective dissolution of irradiated fuel would provide robust separations that are not hampered by interfacial constraints.

2. Characterize the behaviors of competing ions during crystallization. Although the actinyl(VI) cations are chemically and structurally unique in the periodic table, other elements can co-precipitate with them. Systematic studies of the structures, energetics, and kinetics of the co-crystallization of impurities in these solids will broaden the application space for such separations and set boundaries for process intensification by precipitation.

3. Engineer continuous crystallization processes. Precipitative separations are generally straightforward to implement in batch-based separation systems, although continuous separations are often highly desirable. Can the required degree of simplification, process intensification, and product characteristics be achieved in continuous precipitative separations from the complex milieu of nuclear fuel?

4. Design tailored crystallization agents. Temperature swings constitute a major tool for controlling solubility, and addition of crystallization agents can selectively precipitate target species. Such 
additives can cause the precipitation of known solid phases (e.g., Ba addition for removal of sulfate) but more far reaching would be advances in crystal design using additives tailored to promote extended interactions in engineered lattices.

\subsection{References}

Brown, M. A.; Wardle, K. E.; Lumetta, G.; Gelis, A. V. Accomplishing Equilibrium in ALSEP: Demonstrations of Modified Process Chemistry on 3-D Printed Enhanced Annular Centrifugal Contactors. Procedia Chemistry 2016, 21 167-73.

Burns, J. D.; Moyer, B. A. Group Hexavalent Actinide Separations: A New Approach to Used Nuclear Fuel Recycling. Inorganic Chemistry 2016, 55(17), 8913-19.

Chikazawa, T.; Kikuchi, T. Shibata, A.; Koyama, T.; Homma, S. Batch Crystallization of Uranyl Nitrate. Journal of Nuclear Science and Technology 2008, 45(6), 582-87.

Chowdhury, A. U.; Lin, L.; Doughty, B. Hydrogen-Bond-Driven Chemical Separations: Elucidating the Interfacial Steps of Self-Assembly in Solvent Extraction. ACS Applied Materials \& Interfaces 2020, 12(28), 32119-130.

Custelcean, R. Direct Air Capture of $\mathrm{CO}_{2}$ via Crystal Engineering. Chemical Science 2021, 12(38), $12518-28$.

Danesi, P. R. Solvent Extraction Kinetics. Principles and Practices of Solvent Extraction. J. Rydberg, C. Musikas and G. R. Choppin. New York, Marcel Dekker: 1992, 157-207.

de Gyves, J.; Rodríguez de San Miguel, E. Metal Ion Separations by Supported Liquid Membranes. Industrial \& Engineering Chemistry Research 1999, 38(6), 2182-202.

Donohue, T. Lanthanide Photochemistry Initiated in $f-f$ Transitions. Journal of the American Chemical Society 1978, 100(23), 7411-13.

Donohue, T. Photochemical Separation of Cerium from Rare Earth Mixtures in Aqueous Solution. Chemical Physics Letters 1979, 61(3), 601-604.

Higgins, R. F.; Cheisson, T.; Cole, B. E.; Manor, B. C.; Carroll, P. J.; Schelter, E. J. Magnetic Field Directed Rare-Earth Separations. Angewandte Chemie International Edition 2020, 59(5), 185156.

Hirai, T.; Komasawa, I. Separation of Eu from Sm/Eu/Gd Mixture by Photoreductive Stripping in Solvent Extraction. Industrial \& Engineering Chemistry Research 1995, 34(1), 237-43.

Hua, Y.; Flood, A. H. Flipping the Switch on Chloride Concentrations with a Light-Active Foldamer. Journal of the American Chemical Society 2010, 132(37), 12838-40.

Lantham, W. B.; Runion, T. C. PUREX Process for Plutonium and Uranium Recovery. ORNL-479. Oak Ridge National Laboratory, 1949.

Li, S. X.; Johnson, T. A.; Westphal, B. R.; Goff, K. M.; Benedict, R. W. Electrorefining Experience for Pyrochemical Reprocessing of Spent EBR-II Driver Fuel. INL/CON-05-00305. Idaho National Laboratory, 2005.

Litvina, M. N.; Malikov, D. A.; Maryutina, T. A.; Kulyako, Y. M.; Myasoedov, B. F. Separation of U, Pu, and Am Recovered from Mixed Oxide (MOX) Fuel by Countercurrent Chromatography. Radiochemistry 2007, 49(2), 162-165.

Liu, Z.; Clark, A. E. An Octanol Hinge Opens the Door to Water Transport. Chemical Science 2021, 12(6), 2294-2303. 
Lovering, K.; Nayak, S.; Bu, W.; Uysal, A. The Role of Specific Ion Effects in Ion Transport: The Case of Nitrate and Thiocyanate. Journal of Physical Chemistry C 2020, 124(1), 573-581.

Malval, J.-P.; Gosse, I.; Morand, J.-P.; Lapouyade, R. Photoswitching of Cation Complexation with A Monoaza-Crown Dithienylethene Photochrome. Journal of the American Chemical Society 2002, 124(6), 904-905.

Maryutina, T. A.; Litvina, M. N.; Malikov, D. A.; Spivakov, B. Y.; Myasoedov, B. F.; Lecomte, M.; Hill, C.; Madic, C. Multistage Extraction Separation of Am(III) and Cm(III) in Planet Centrifuges. Radiochemistry 2004, 46(6), 596-602.

Mukherjee, S.; Zaworotko, M. J. Crystal Engineering of Hybrid Coordination Networks: From Form to Function. Trends in Chemistry 2020, 2(6), 506-18.

Salpage, S. R.; Lanzetta, R. C.; Zhou, Y.; Wang, J. C.; Albrecht-Schmitt, T. E.; Hanson, K. Wavelength Selective Separation of Metal Ions Using Electroactive Ligands. Chemical Communications 2018, 54(54), 7507-10.

Servis, M. J.; Clark, A. E. Surfactant-Enhanced Heterogeneity of the Aqueous Interface Drives Water Extraction into Organic Solvents. Physical Chemistry Chemical Physics 2019, 21(6), 2866-74.

Servis, M. J.; McCue, A; Casella, A. J.; Clark, A. E. The Role of Surfactant Force Field on the Properties of Liquid/Liquid Interfaces. Fluid Phase Equilibria 2020, 511, 112497.

Stockmann, T. J.; Ding, Z. Uranyl Ion Extraction with Conventional PUREX/TRUEX Ligands Assessed by Electroanalytical Chemistry at Micro Liquid/Liquid Interfaces. Analytical Chemistry 2011, 83(19), 7542-49.

Wall, T. F.; Jan, S.; Autillo, M.; Nash, K. L.; Guerin, L.; Naour, C. L.; Moisy, P.; Berthon, C. Paramagnetism of Aqueous Actinide Cations. Part I: Perchloric Acid Media. Inorganic Chemistry 2014, 53(5): 2450-59.

Willberger, C.; Amayri, S.; Häußler, V.; Scholze, R.; Reich, T. Investigation of the Electrophoretic Mobility of the Actinides Th, U, Np, Pu, and Am in Different Oxidation States. Analytical Chemistry 2019, 91(18), 11537-43. 


\title{
6. FRD 5: Exploit Principles of Coordination Chemistry to Simplify Actinide Separations
}

\begin{abstract}
This FRD highlights the value of coordination chemistry in actinide separations. Many separative strategies are available for actinide recovery, and many prioritize excellent product purity and yields. Recently, the economic feasibility of actinide recycling has become the main determinant, with process simplification at the forefront of R\&D efforts. The design of materials and molecules is integral to finding novel approaches in actinide separations that allow significant process simplification. The discovery efforts must consider six important features of a promising coordination environment as compatibility, selectivity, affinity, kinetics, stability, and predictability are all important physicochemical properties that contribute to process development. The identified challenges are (1) improve the understanding of $f$ element selectivity, (2) accelerate ligand design, (3) manage fission products, (4) predict reagent stability, which all must deliver (5) overall process simplification. A well-coordinated link between the experiment and theory can further advance the theoretical description of actinide binding, benefiting from data science approach. Machine learning (ML) tools, theoretical modeling, and highthroughput synthesis and screening will open new approaches to intelligent ligand design and chemical separations, sampling of vast areas of chemical space. Research needs are recognized for understanding and exploiting the coordination chemistries of actinides and $\mathrm{Ru}$, $\mathrm{Zr}, \mathrm{Nb}, \mathrm{I}$, and $\mathrm{Tc}$ in nuclear fuel cycles, as management of those particular fission products is most problematic to actinide separations. Novel actinide coordination environments will inherently sustain chemical and radiolytic degradation. Understanding such degradation processes must be mastered at the molecular level by developing recommendations for reagent classes and strategies to build in robustness. Such R\&D efforts may also be uniquely transformed with a data science approach, computations, and high-throughput synthesis and screening.
\end{abstract}

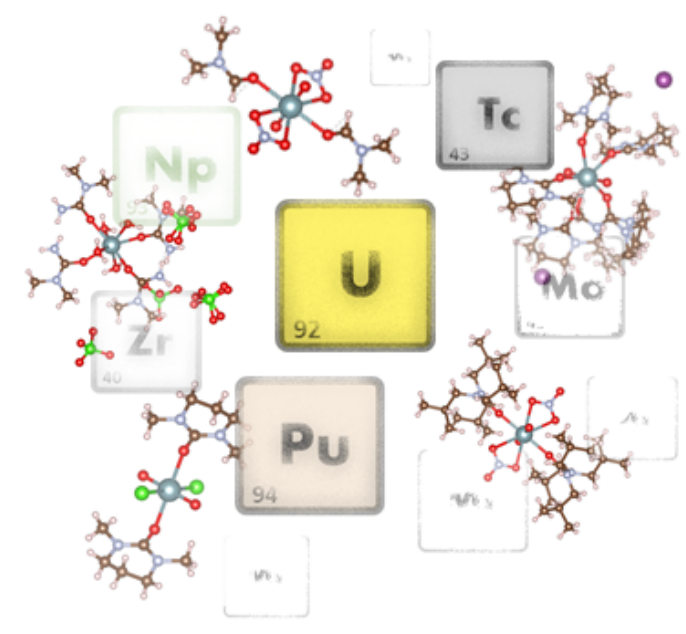

A graphical allegory for actinide separations challenge in coordination chemistry. The shown metal complexes are crystallographic structures of uranyl cation coordinated by monoamide reagents available via Cambridge Structural Database. (Courtesy of INL.)

\subsection{Introduction}

Coordination chemistry is fundamentally central to $f$-element separations at the front and back end of the nuclear fuel cycle. Its principles, as defined by Alfred Werner in the late 19th century, have universally led chemists to isolate metal ions, whether guided by directionality of orbital overlap or electrostatic efficiency of ion packing. The physicochemical properties of a hydrated metal ion change when its coordination sphere is perturbed by electron donors. The enthalpic and entropic contributions to a net energy balance for this reaction are defined by chemical bonding and solvation forces, largely driven by the nature of the metal and the donor atoms of coordinating reagent. Such metal ligation offers the opportunity to discriminate metal ions simply by variation of thermodynamic stabilities of formed complexes, adducts, or ion pairs. This principle applied in liquid-liquid and solid-liquid separative technologies aids the recovery of metal ions from matrices as complex as mineral ores or irradiated nuclear fuel. 
Implementation of actinide coordination chemistry in nuclear fuel cycle separations has adapted two distinct tactics that are rooted to $f$-element electronic structure. One exploits the diverse redox chemistry of the early actinides, and the other targets $5 f$ electron delocalization and energy degeneracy-driven mixing of $5 f$ and ligand orbitals (Choppin and Jensen 2006). Relative to the rich $d$-block element chemistry, driven by ligand field stabilization and covalent bonding, the actinides are less chemically active. The electrons filling the $5 f$ orbitals are shielded by $6 d$ and $7 s$ orbitals and rarely participate in chemical bonding. For early actinides, relativistic effects and ineffective screening of valence electrons from the nucleus changes the relative energy differences between the $5 f$ and $6 d$ orbitals, inviting electron transfer and yielding multiple valence states (Kaltsoyannis et al. 2006). Accordingly, manipulation of actinide redox states has proven a suitable option in the purification of $\mathrm{U}$ in the PUREX process, where the reduction of $\mathrm{Pu}$ and $\mathrm{Np}$ is a key component. This redox-based coordination chemistry discriminates actinides based on the electrostatic binding with TBP as guided by the charge density of the metal ion. Today, the PUREX solvent extraction process remains the state of art in nuclear fuel cycle separations (Appendix C).

For heavier actinides, as the electrons continue to fill the $5 f$ orbitals, the energy gap between the $5 f$ and $6 d$ orbitals increases, and a +3 oxidation state gains prevalence. The inept screening of outer electrons from nucleus by the embedded $f$ electrons contracts the ionic radius, and consequently the coordination chemistry of trivalent actinides resembles the members of $4 f$ series. The isomorphic chemistry of $4 f$ and $5 f$ elements renders the isolation of trivalent minor actinides from UNF effluents a challenging task, yet to be implemented on the industrial scale. Nonetheless, numerous separation concepts have been developed, with majority exploiting a generally accepted theory of $5 f$ orbital delocalization (Nash et al. 2006).

Trivalent $f$-elements and Lewis base ligands interact by forming ionic bonds because $f$ electrons are buried deep inside the electron cloud and their participation in bond formation is minimal. For actinides the electrons in the $5 f$ orbitals are further from the nucleus, relative to $4 f$, and this larger radial extension makes the valence electrons more available to bond formation. Accordingly, when atomic orbitals locate some symmetric and energetic compatibility, some $5 f$ character may be instilled into the molecular orbitals of the complex. Little spatial overlap results from such $5 f$ electron delocalization, however, and a perturbation of internuclear electron density is rather small. A greater participation of $5 f$ orbitals in bonding compared to $4 f$ has been linked with energy degeneracy-driven mixing of $5 f$ and ligand orbitals, which primarily occurs when actinides are coordinated by reagents containing chlorine, nitrogen, sulfur, or selenium donor atoms (Su 2018). The inclusion of such soft donor atoms in the metal coordination sphere, in most cases, increases the stability of the actinide complexes relative to trivalent lanthanides of equivalent charge density. Thus, the use of ligand structures that include such soft donor atoms was adapted as a successful coordination chemistry tactic in nuclear fuel cycle separations.

\subsection{Current State of Coordination Chemistry in Actinide Separations}

\subsubsection{Organophosphates}

Neutral and acidic organophosphorus compounds are the most widely investigated reagents for the separation of actinides. The clear standouts in this class of coordination agents are TBP and di(2ethylhexyl)phosphoric acid (HDEHP). The structures are shown in Scheme 6.1. TBP is very effective at solvating major actinides $(\mathrm{U}, \mathrm{Pu})$ in the organic environment, while metal ions with effective charge of +3 or less remain in the aqueous phase. The coordination chemistry is guided by the charge density of the metal, and the $\mathrm{U}$ product is isolated after redox adjustment significantly lowers the extractability of $\mathrm{Np}(\mathrm{V})$ and $\mathrm{Pu}(\mathrm{III})$. Despite its often cumbersome and unstable redox chemistry, the described PUREX process remains the state of art as the solvent extraction-based actinide separation process implemented on the industrial scale (see Appendix C). 


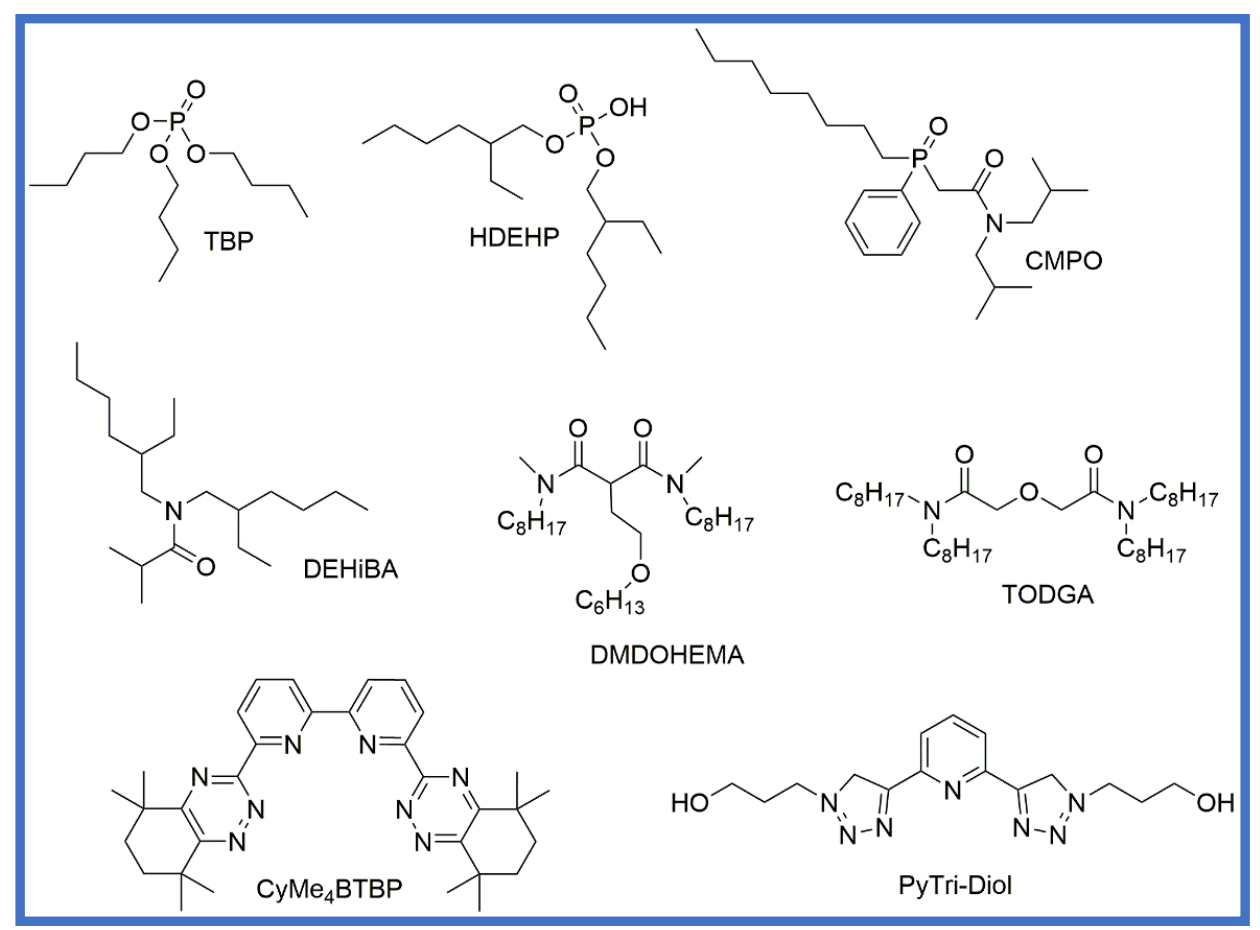

Scheme 6.1. Representative structures of various ligands utilized in liquid-liquid processes developed to isolate actinides from UNF. (Courtesy of INL.)

The strong cation exchanger HDEHP dimerizes in organic solvents. The dimers partially open upon the coordination of a charged metal ion and the dissociated hydrogen ions return to aqueous phase, maintaining charge neutrality. The ion exchanging mechanism of liquid-liquid partitioning of metals is sustained through an acid/base equilibrium. Accordingly, the extraction of metal ions occurs in mildly acidic aqueous mixtures, where deprotonation of a phosphoric acid is feasible. Extractants such as HDEHP were originally studied for rare earth beneficiation as the efficient coordination of trivalent $f$-elements yields dramatic variability in the complexation strength. For example, the extractability of La(III) by HDEHP is about a million times less efficient relative to Lu(III). A variety of phosphoric, phosphonic, and phosphinic acid reagents have shown utility in metal ion separations spanning broad application spectrum.

The most recognizable utility of acidic organophosphates at the back end of nuclear fuel cycle is the resourceful liquid-liquid balance of HDEHP and aminopolycarboxylate chelator $\mathrm{H}_{5} \mathrm{dtpa}$ as developed for the TALSPEAK process (Moyer et al. 2015). The chemistry of TALSPEAK is rather slow to reach the liquid-liquid partitioning equilibrium, which is considered a serious drawback. The chemistry is typically implemented at $\mathrm{pH}>3$ conditions to weaken the competition to an aminopolycarboxylate chelator from the ligand protonation equilibria. The octadentate $\mathrm{H}_{5} \mathrm{dtpa}$ complexant coordinates the metal ion, displacing all but single water of hydration. As the metal ion is well-shielded from the interactions with the water molecules in the bulk aqueous medium, the slow metal exchange results in significant kinetic inertness. The dissociation of such $f$-element/aminopolycarboxylate complexes is promoted by hydrogen ions, and the kinetic limitations are especially visible in aqueous mixtures with low proton content. To circumvent this high kinetic barrier, either high concentration of aqueous buffer must be present, or more acidic aqueous chelator must be used.

Other organophosphate compounds deserving of note have found use in trivalent $f$-element coextraction. Neutral solvating ligands, such as octyl-(phenyl)- $N, N$-diisobutylcarbamoylmethylphosphine oxide (CMPO) (Scheme 6.1), as a component of the transuranic extraction (TRUEX) solvent (Moyer et al. 2015). This reagent links a metal coordinating affinity of a phosphoryl and a carbonyl functionality into a bidentate 
structure. This configuration offers strong affinity for trivalent actinide coordination in highly acidic media, which renders the back-extraction of actinide ions rather complicated.

\subsubsection{Monoamides}

Although uranyl solvating ability of neutral organophosphates is unrivaled to this day, amide neutraloxygen-donor extractants have emerged as useful alternatives. High loading capacity, excellent solvent hydrodynamic properties, and ease of removal of degradation products are a few notable advantages of the organophosphorus ligands. However, disadvantages to the use of TBP include disposition of the secondary waste stream generated by thermal treatment of the spent solvent and the deleterious influence of the primary degradation products on process performance. Monoamide extractants offer a promising alternative for $\mathrm{U}$ and $\mathrm{Pu}$ recovery from UNF (Authen et al. 2021). These extractants adhere to a solvating mechanism of metal extraction that dominates when $\left[\mathrm{HNO}_{3}\right]<4 \mathrm{M}$. Monoamides show good U/Pu selectivity at moderate acidity and reduce complexity by using a reagent that leaves no solid waste when incinerated (CHON principle), and their hydrolytic and radiolytic degradation products are generally less detrimental to process performance compared to those of TBP. In contrast, monoamides are less basic relative to phosphates, which is manifested in lower extraction affinity for $\mathrm{U}$.

A very attractive feature of the coordination chemistry of the monoamide extractant class is the steric hindrance introduced by branched alkyl substituents. A monoamide reagent complexes a linear dioxouranyl cation by forming $\mathrm{UO}_{2}\left(\mathrm{NO}_{3}\right)_{2}(\mathrm{MA})_{2}$ structures $(\mathrm{MA}=$ monoamide), and a spherical tetravalent $\mathrm{Pu}$ is predominantly coordinated as $\mathrm{Pu}\left(\mathrm{NO}_{3}\right)_{4}(\mathrm{MA})_{2}$. The $\mathrm{Pu}$ extraction dependency on the nitrate ion concentration is much more pronounced relative to $\mathrm{U}$, which enables the control of $\mathrm{U} / \mathrm{Pu}$ selectivity via a simple shift of aqueous acidity without the use of often unstable redox active reagents. The increased steric crowding around $\mathrm{Pu}(\mathrm{IV})$ is intensified by the presence of four bidentate nitrates, relative to two surrounding $\mathrm{U}^{\mathrm{VI}} \mathrm{O}_{2}{ }^{2+}$ cation. Such saturated coordination environment of the metal is sensitized to minor structural changes such as $n$-propyl for iso-propyl substitution reported for MA extractants. As a result, the extraction affinity for $\mathrm{Pu}$ is significantly lower relative to $\mathrm{U}$ when branched monoamide reagents such as $N, N$-di-2ethylhexylisobutyramide (DEHiBA) are used. The structure of DEHiBA is shown in Scheme 6.1.

\subsubsection{Diamides}

In the realm of solvating extractants developed for minor actinide separations, the malonamide class of coordination agents has gained interest in the past (Modolo et al. 2015). The presence of two carbonyl oxygens separated by a methylene bridge enables the formation of stable six-membered chelate rings upon the coordination of metals. This structural feature enables the extraction for trivalent $f$-elements at moderate $\mathrm{HNO}_{3}$ concentrations. The diamide extraction (DIAMEX) process, developed in France, uses malonamides to coextract trivalent $4 f$ and $5 f$ metals from liquid raffinates, resulting from the initial removal of bulk $\mathrm{U}$ from dissolved UNF. The structure of a most-often used malonamide reagent, $N, N^{\prime}$-dimethyl- $N, N^{\prime}$ dioctylhexylethoxymalonamide (DMDOHEMA) is shown in Scheme 6.1. One limitation of malonamide facilitated metal extraction is the low affinity for metal coordination, requiring high ligand content in the

process. In part owing to conformation strain, this limitation can be overcome through molecular design of highly constrained derivatives (Lumetta et al. 2003).

\subsubsection{Diglycolamides}

Relative to malonamides, the increased efficiency of trivalent $f$-element binding was realized with the replacement of the methylene bridge by a $-\mathrm{CH}_{2}-\mathrm{O}-\mathrm{CH}_{2}-$ linker with a now very frequently used diglycolamide class of extraction agents. Solvation of trivalent $f$-elements in nonaqueous environments typically uses two diglycolamide molecules and inorganic anions such as nitrate are coextracted to balance the charge of the metal ion. Scheme 6.1 shows the structure of $N, N, N^{\prime}, N^{\prime}$-tetraoctyldiglycolamide 
(TODGA), one of the most recognizable reagents used in $f$-element separations. Although the efficient extraction of trivalent $f$-elements has paved way to several renditions of $f$-element coextraction concept, the diglycolamide coordination chemistry suffers from the propensity to form third phases in aliphatic diluents. This limitation is circumvented by the addition of TBP, which improves the physical properties of the organic formulation. Recently, TBP has been replaced by the simple presence of 5 vol.\% of 1-octanol to adhere to the CHON principle. Since 2001, diglycolamides became synonymous with actinide separations from aqueous raffinates resulting from the initial step of bulk $\mathrm{U}$ isolation, supporting solvent formulations for processes like $i$-SANEX, European group actinide extraction (Euro-GANEX), European extraction of americium (Euro-EXAm), and ALSEP (Modolo et al. 2015; Moyer et al. 2015).

\subsubsection{Bis-triazinyl-Pyridines, Bis-Triazinyl-Bipyridines and Bis-Triazolylpyridines}

The coextraction of trivalent $f$-elements carries a preconceived strategy to differentiate the members of the $4 f$ and $5 f$ series in a final separation step. One option for this challenging task is the highlighted coordination chemistry of TALSPEAK. Another alternative, built around the CHON requirement, uses the coordination pocket of the nitrogen heterocycle chemistry to sustain $f$-element coordination in aqueous mixtures containing high concentrations of hydrogen ion. The partitioning of metal ions facilitated by such reagents is driven by the solvating mechanism relative to the ion-exchange mechanism of TALSPEAK. Accordingly, the solvation of metals is favored when aqueous content of nitrate is high. Beginning with bis-triazinylpyridine structures, the presence of soft-donor $\mathrm{N}$ is manifested by promising $5 f / 4 f$ separation factors even from highly acidic aqueous solution. The softness of the heterocycle chemistry was further improved when bis-triazinyl-bipyridines introduced a fourth nitrogen into the coordination sphere. Such encouraging results began extensive structure-function development work culminating with $\mathrm{CyMe}_{4}-\mathrm{BTBP}$ (Scheme 6.1), a molecule central to the 1-cycle-SANEX (selective actinide extraction) and CHALMEX (Chalmers extraction) processes (Modolo et al. 2015). An attempt to overcome the phase-transfer kinetic shortcomings of $\mathrm{CyMe}_{4}-\mathrm{BTBP}$ class using a preorganized cis-conformation of $\mathrm{CyMe}_{4}-\mathrm{BTPhen}$ resulted in overcompensated complexation strength, which significantly impeded the ability to recover the extracted actinides.

The $f$-element coordination chemistry of $\mathrm{N}$ heterocycles also found utility in aqueous complexation (i.e., extraction holdback complexants). Several structures were functionalized with sulphophenyl moieties, yielding good aqueous solubility. The opportunity to apply such ligands in a TALSPEAK-like function proved valuable when paired with TODGA, yielding a scenario where a complete isolation of Am away from $4 f$ elements and $\mathrm{Cm}$ is feasible. Further development of aqueous coordination reagents from the Nheterocycle family resulted in structures such as PyTri-Diol, a member of the bis-triazolylpyridine family (Scheme 6.1) that supports a complete differentiation of trivalent actinides from trivalent lanthanides in $0.4 \mathrm{M} \mathrm{HNO}_{3}$ (Geist and Panak 2021).

\subsubsection{Aqueous Complexants}

Although the $f$-element separations research has been dominated by the development of nonaqueous extraction reagents, the aqueous complexation chemistry has also played an important role. As described for TALSPEAK, a thermodynamic balance between the coordination chemistries in both liquid immiscible phases may afford a very efficient separation option. Water-soluble coordination agents have also been used as complexants for actinides with readily accessible redox potentials. The use of acetohydroxamic acid as reduction/complexation reagent as means to separate $\mathrm{Pu}$ from $\mathrm{U}$ is a prime example. Further, aqueous coordination chemistry is often the best tactic to mask problematic FPs to prevent their coextraction with actinides. The management of $\mathrm{Zr}, \mathrm{Mo}, \mathrm{Tc}$, and Pt-group metals has used aqueous complexants with varying success. The use of 1,2-cyclohexane- $N, N, N^{\prime}, N^{\prime}$-ethylenediaminetetraacetic acid to control the partitioning of $\mathrm{Zr}$ from PUREX-like raffinates is one example (Authen et al. 2021). 


\subsubsection{Polymer-Supported Complexants}

Numerous actinide coordination agents have also been immobilized onto crosslinked polymer materials such as polystyrene or poly(glycidyl methacrylate) to generate a solid-liquid metal ion separation platform. Ligands are covalently linked to the polymeric material most often via a structural component that does not participate in metal ion coordination. Alternatively, extraction chromatographic materials use hydrophobic forces and capillarity to adhere lipophilic ligands onto the solid support surfaces such as crosslinked polystyrene divinylbenzene matrix or inert methacrylic polymeric support. The immobilization does not inhibit the complexation chemistry, and chromatographic application of these materials offers an attractive technology to sorb metal ions from dilute aqueous streams.

\subsubsection{Inorganic Ion-Exchange Materials}

Clays are nature's basic inorganic ion-exchanging materials, built based on aluminosilicate minerals where sodium ions are released upon uptake of metal ions. Because of the potential to gain excellent chemical, mechanical, and thermal stability, inorganic ion-exchange materials are attractive for capture of radioactive metal ions. Metal adsorption selectivity rules for such inorganic materials are not solely determined by the electrostatic binding of the exchanging ions. Surface versus pore adhesion, solvent interactions, intramolecular rearrangement, sorption dynamics

$$
\text { a }
$$

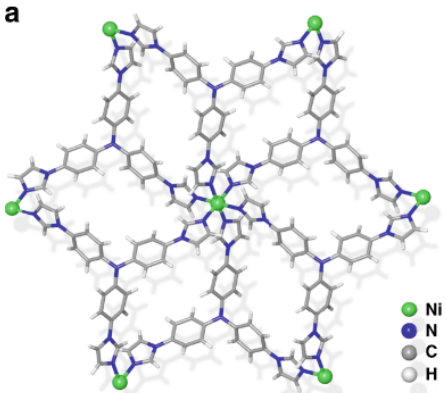

c

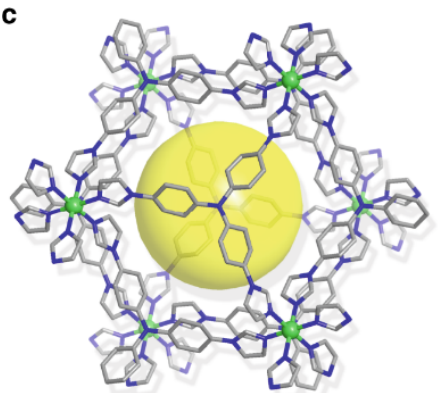

d

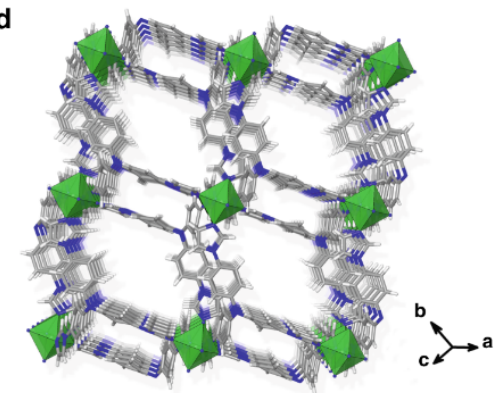

b

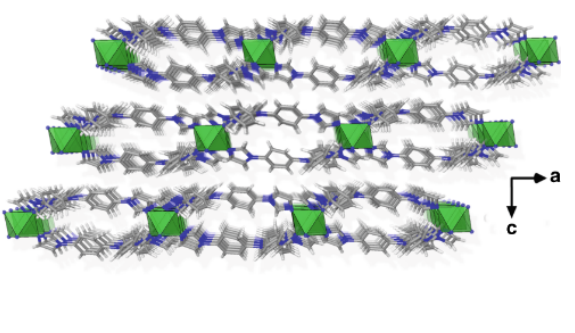

Figure 6.1. Example of nickel-based cationic MOF material developed for ${ }^{99} \mathrm{TcO}_{4}-$ uptake. (Courtesy of INL.)

\section{and loading capacity are all factors} $\varliminf_{c}^{a}$

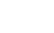

influencing the selectivity of inorganic ion-exchange materials. In general, the inorganic crystalline structure, the porosity and counterions can all be tuned to find optimal metal adsorption characteristics depending on the application. Examples of inorganic sorbent materials developed for nuclear applications include ammonium molybdophosphates and monosodium titanates utilized for capture of ${ }^{137} \mathrm{Cs}$ and ${ }^{90} \mathrm{Sr}$ from contaminated waters, layered metal sulfides for U uptake from seawater or MOFs developed for technetium uptake as shown in Figure 6.1 (Shen et al. 2020). In many cases, the inorganic ion exchangers are embedded in the polymer matrix to improve their mechanical properties.

\subsubsection{Six pillars of Ligand Development}

An 80-year evolution of coordination chemistry in actinide separation science has identified six essential pillars that serve as a foundation for a successfully applied ligand (Geist and Panak 2021). Pillar 1 is compatibility. A coordination agent and its metal complexes must be sufficiently soluble in operating liquid phases, ensuring the solvation and physical (hydrodynamic) properties of the solvent and a metalloaded environment are not drastically altered. Pillar 2 is selectivity. When using the principles of coordination chemistry to separate species, the net energy difference in the system must be attained. This differential may be reached via complex stability, solvation forces, or equilibrium gradients - all inherently dependent on the structure of the metal coordination agent. Pillar 3 is affinity to sustain metal complexation. 
The efficient product isolation is complete upon its recovery from a working extraction phase. Such a solvent stripping step is attained when a modification of chemical environment alters the reagent's coordination prowess. Some convenient routes to manipulation of ligand affinity are simple acid or salt concentration swings. Pillar 4 is kinetics. The timescale of chemical equilibrium must be compatible with the technology applied to accomplish the separation. Pillar 5 is stability. The hydrolytic and radiolytic stability is a vital prerequisite for application of each coordination reagent in actinide separation. Furthermore, attention must be given to ligand degradation mechanisms, prioritizing structures leading to innocuous decomposition products. Pillar 6 is predictability. The influence of novel coordination chemistry as applied in a separation process may be derived to predict the mass balance at equilibrium. The ability to accurately model the separation outcomes is invaluable to advancing the TRL and material accountability for actinide separation processes.

\subsection{Research Needs to Advance the Application of Coordination Chemistry}

The principles of coordination chemistry as applied to simplified and compact actinide separations must adhere to the six pillars of ligand development. Accordingly, longstanding or emerging research needs for actinide separations may be evaluated based on the limitations imposed by those six essential characteristics. With this in mind, the following general research needs are identified.

\subsubsection{Understanding/Prediction of Selectivity}

Quantitative grasp of actinide coordination remains a great challenge. Improved accuracy of quantum chemical description of electronic structure in coordination spheres of $5 f$ ions holds the key to further advancement of actinide separations. Currently, difficulties to accurately embody solvation effects into the complexation free energies and limitations of conventional density functional theory (DFT) methods in the description of open $\mathrm{f}$ shells in lanthanides and actinides spur controversies over the origin of selectivity in $f$-elements. Constant improvement of theoretical models, aided by the utility of machine learning tools, and guided collection of experimental data will lead to more accurate theoretical descriptions of ligand binding and selectivity to guide molecular design efforts.

\subsubsection{Accelerate Ligand/Adsorbent Design with Integrated High-Throughput Synthesis, Experimental Evaluation, High-Performance Computing, and Data Science Approach}

A balance between ligand design, ligand synthesis, and ligand testing is often difficult to find in $f$-element separations. Empirical structure-function approaches dominate the research space. New approaches to intelligent ligand design and chemical separations may combine machine learning tools, theoretical modeling, and high-throughput synthesis and screening to accelerate sampling of vast areas of chemical space. This approach may implement various strategies in coordination chemistry such as redox couples, covalency, ion pair formation, anion and long-range solvent effects, and external energy fields.

\section{FP management}

The coordination of $\mathrm{U}$ and $\mathrm{Pu}$ cations is stronger with hard-donor solvating extractants such as TBP and $\mathrm{DEH} i \mathrm{BA}$, relative to most FPs. As a result, most FPs do not appreciably interfere in the isolation of actinides, except for ruthenium, zirconium, niobium as well as gaseous ${ }^{129} \mathrm{I}$ and potentially volatile ${ }^{99} \mathrm{Tc}$. The chemistry of ruthenium in nitric acid is complicated by its complex and unpredictable speciation, showing presence of nitroso-, nitrato-, and hydroxy- species, with an added volatile nature of its tetraoxide. Zirconium and niobium show similar chemical behavior, forming anionic nitrato- species in nitric acid. Iodine's reactivity with hydrocarbons necessitates the efforts of its head-end removal, prior to introduction of nonaqueous solvents. In nitric acid, Tc exists predominantly as a charge-diffuse monovalent pertechnetate anion $\left(\mathrm{TcO}_{4}^{-}\right)$. Because of its lower hydration energy compared to nitrate, $\mathrm{TcO}_{4}^{-}$often replaces 
$\mathrm{NO}_{3}{ }^{-}$in nonaqueous solvates. The unique chemistries of these elements offer opportunities to seek novel coordination environments for FP control in actinide separations.

\section{Quantitative prediction of stability in liquid-liquid and solid-liquid systems}

As with ligand design, a less empirical approach in studies of material degradation will benefit coordination-driven actinide separation technologies. Elucidation of fundamental radiolytic parameters (degradation yields, reaction kinetic rates, and particle and energy deposition dependence) for molecular building blocks and reagent classes can be fundamentally transformed with data-science approach, computations, and high-throughput synthesis and screening.

\section{Simplification}

Actinide recycling has relatively low impact $(\sim 3 \%)$ on the overall final cost of nuclear electricity (Poinssott et al. 2015). However, the role of recycling in the socioeconomic appraisal of sustainability of this energy source is imperative. Sustainable advanced fuel cycle options must offer better utilization of natural U resources, which is already accomplished in a monorecycle of $U$ and $\mathrm{Pu}$. Further utilization of these actinide resources may be realized through multirecycling options, adding further benefits of reduced proliferation risks associated with $U$ enrichment and significantly lowering of nuclear waste lifetime to a manageable 300 years. Ultimately, a sustainable energy source must be economically viable, which stresses the importance of simplification of separation chemistries through improved efficiency, robustness, and safety. As novel coordination environments and separation flowsheets continue to evolve, facing higher burnup fuels and increased presence of minor actinides, simplified approaches will incentivize multirecycling options and advance the aspirations of sustainability.

The following new research directions are proposed to address these generalized research needs.

\subsubsection{Coordination-Driven Actinide Control}

The current state of actinide coordination chemistry emphasizes the importance of the development of novel reagents to address all technical challenges of actinide separations. Decades of developmental research, structure-function relationship studies, and refinement of separation flowsheets have enabled efficient options for actinide recycling at the back end of nuclear fuel cycle (Modolo et al. 2015; Moyer et al. 2015; Authen et al. 2021). Although it is imperative to support the expansion of the scope of actinide separations to studies addressing molecular interactions at the interfaces, soft-matter reactivity, and external fielddriven phenomena (see FRD 4), the manipulation of the ligand-based selectivity and affinity for solutes will continue to drive the discovery of novel actinide separation strategies. The electronic configuration in a chemical bond can be guided in many ways enroute to new, highly simplified sequestration of actinide ions and problematic FPs. New patterns of selectivity can be discovered through the following concepts:

- Ligand-induced variation of redox chemistry

- Bonding and steric geometric effects

- Coordination-driven covalency control

- Multifunctional ligands, heterobimetallic coordination spheres. 


\section{Core Idea}

Design and synthesis of new extractants will continue to drive the field of actinide separations. Exploiting a combination of timeresolved computational modeling, highthroughput synthetic methodologies, and uniform screening is coupled with machinelearning to accelerate ligand discovery.
The discovery of novel molecular systems must encompass advanced approaches to intelligent ligand design for chemical separations, where a combination of time-resolved computational modeling, high-throughput synthetic methodologies, and uniform screening is coupled with machine-learning enabled workflows. Although more accurate theoretical descriptions of ligand binding and selectivity are needed, novel computational approaches to predicting hydrodynamic properties of operating liquid phases will be of great

value. The synthesis and screening of new ligands may be potentially accelerated with robofluidic technologies such as high-throughput autonomous flow reactors, which allow in situ analyses. Further, a variety of data mining tools may be implemented to survey literature for applicable metrics (experiment and theory) to enable the discovery of novel ligand classes. The reagent design should adhere to the six pillars of ligand development, anticipating the challenges posed by the increasing content of minor actinides in the higher burnup of PWR and BWR used fuel and Pu in MOX and advanced nuclear fuels.

\subsubsection{Anion Recognition}

Anionic FPs and metallate complexes of actinides present quite different coordination challenges than encountered with cations. One common case made for anion recognition in advanced fuel cycle separations derives from $\mathrm{U}$ fission production of long-lived ${ }^{99} \mathrm{Tc}$, which speciates ordinarily as pertechnetate, ${ }^{99} \mathrm{TcO}_{4}{ }^{-}$ (Thevenet et al. 2021). Similar rationale arises for longlived ${ }^{129} \mathrm{I}^{-}$. "Off-target" nonradioactive anions (e.g., nitrate, nitrite, hydroxide, sulfate, aluminate) also need

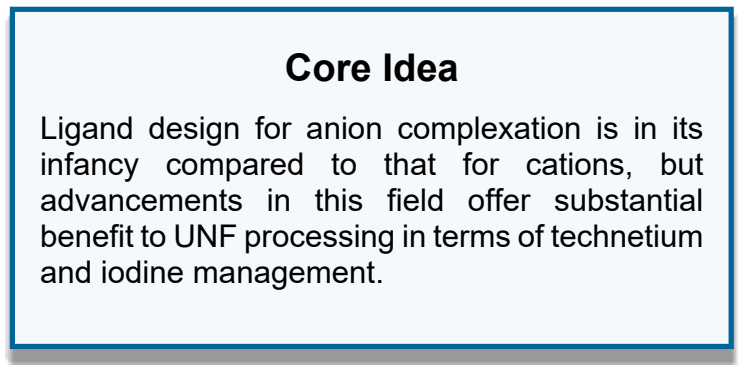
to be managed (Moyer et al. 2013). Recognition using selective receptors can add specificity to separations. Receptor design has traditionally sought affinity and selectivity using control of inner-sphere coordination based on hydrogen-bond-donor receptors. The secondary coordination sphere is now getting attention, and cooperativity can emerge to effect novel binding characteristics. Switchable receptors (e.g., using light) are being investigated for regeneration (Hua and Flood 2010). Liquid-liquid extraction and crystallization are common separation methods.

The principles of anion recognition (Sessler et al. 2006) parallel cation coordination with key differences. Anions are larger in size (e.g., $4.5 \AA$ for $\mathrm{TcO}_{4}^{-}$), and because they are charge-diffuse, their affinities are much lower than transition metals (possibly closer to alkali cations). Receptors are usually larger and composed of many neutral or charged $\mathrm{H}$ - and halogen-bonding sites. Amides and secondary amine $\mathrm{NH}$ donors, pyrrole $\mathrm{NH}$ donors and triazole $\mathrm{CH}$ donors, cationic imidazolium, triazololium, guanidinium, and pyridinium are common. There is greater reliance on synthesis to achieve preorganized size/shape complementarity for selectivity and add many binding sites for affinity. Geometrical effects are less stringent; large anions are often rotationally averaged. (De)solvation of the receptor, anion, and complex can help or hinder recognition.

To achieve selectivity, the principles of complementarity dictate size and shape matching as well as use of chemically specific, noncovalent contacts. Going beyond simple 1:1 complexes to assemblies, cooperativity mediated by secondary structures are emerging as key design features. Cooperativity can often lead to either enhanced or novel selectivity. 
The challenge for anion recognition in advanced fuel cycle separations is learning how to design separation systems to recognize anions selectively from a complex milieu with efficient system regeneration. This must start with clear identification of known on-target anions (e.g., ${ }^{99} \mathrm{TcO}_{4}^{-},{ }^{129} \mathrm{I}^{-}$), other on-target species (e.g., metal-based anionic complexes $\left[\mathrm{Pu}(\mathrm{OH})_{6}\right]^{2-}$ ), and off-target anions (e.g., $\mathrm{CrO}_{4}{ }^{2-}$ ) to provide relevant separation goals. Identifying what the concentration levels of these anions are and establishing the identity of interfering anions is necessary to determine whether high-affinity, high-selectivity, or high-affinity and high-selectivity receptors are required. Challenges in receptor design lie in their tailored design for affinity and selectivity and to go beyond the primary coordination sphere to leverage cooperative effects (e.g., in ion pairing, self-assembly, folding, solvation effects, and stimuli-responsive systems for regeneration).

Research needs include advanced approaches to intelligent receptor design for separations. Given that synthesis remains integral and the inner coordination sphere is increasingly understood, we need to research strategies to leverage computational modeling to accelerate de novo design and testing. We need to go beyond inner-sphere coordination to secondary contacts, structures, and solvation. These include efforts to explore and leverage cooperative effects, as they emerge during higher-order self-assembly and in ion-pair binding (possibly for coextraction of radioactive cations and anions by coupling anion receptors to ligands to help simplify separations). We need to understand the role of inner/outer-sphere anions in metal complexes and leverage it for simpler separations and learn how to use external stimuli (light, electric, magnetic) to control anion-based separations for regeneration both in equilibrium and nonequilibrium systems. Regeneration requires an approach that considers the full cycle, which predicates bigger efforts such as team-based science.

\subsubsection{Data Science in Ligand Design}

\section{Core Idea}

Data science offers the possibility of discovering new ligand systems by eliminating human biases in the choice of binding moieties.
Application of modern data science has the potential to drastically increase separation efficiency by improving ligand design through the unbiased exploration of a vast chemical space (see Chapter 9). Combining data science with high-throughput theoretical simulation will enable screening of millions of ligands by leveraging state-ofart exascale computing resources. Simulations at various

levels of theory can be carried out, spanning from first-principles electronic structure calculations to interfacial simulations (e.g., using molecular dynamics). These results can then be used to train ML models that will further assist in the exploration of chemical space. Figure 6.2 shows the interplay of these data science components in the design of coordination molecules and materials. Indeed, different ligands likely will form different molecular complexes with actinide in the solution than at interfaces, hence impacting their transport properties. Therefore, separation efficiency is unlikely to be explained by a single factor that can directly be accessed by simulations. Instead, data science can be used to discover hidden correlations between multiple simulation outputs, which can be used to identify more powerful descriptors to characterize separation ligands. These descriptors will then be used to guide the generation of new ligands. The best candidates will then be validated by experimental efforts. The feedback of these experimental results back into the ML model will be critical for their validation and to improve the prediction accuracy of the ML model. 


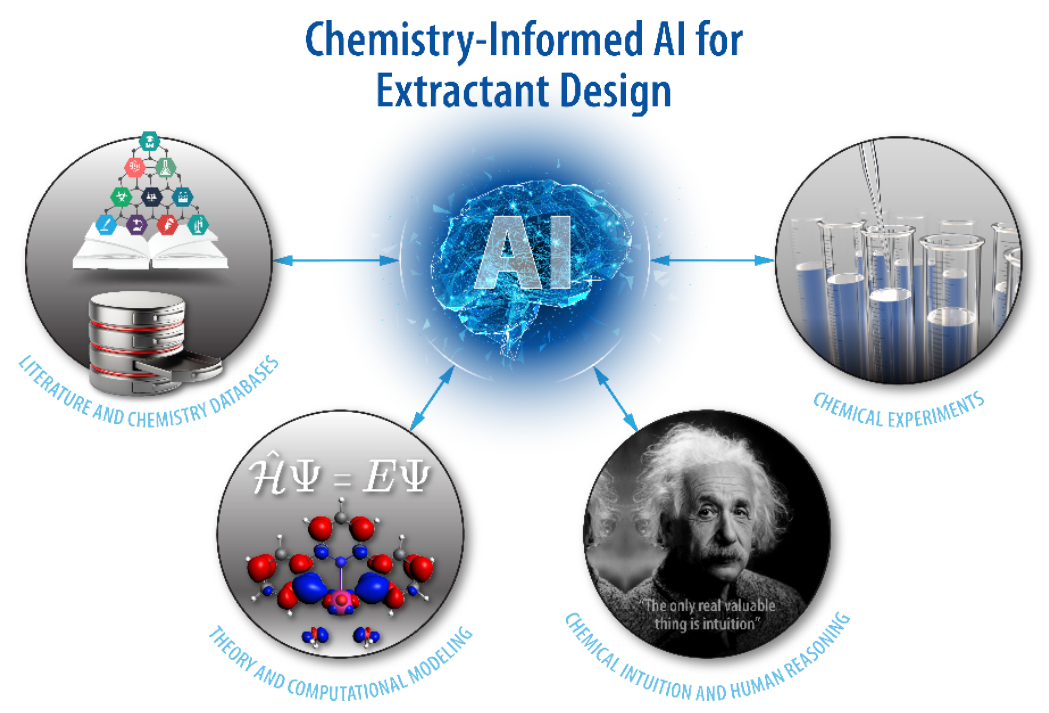

Figure 6.2. Data science in ligand design (Shen 2020, reprinted with permission from Springer Nature).

\subsubsection{Inorganic Ion-Exchange Materials}

Ion-exchange technologies using inorganic materials can offer robustness, good selectivity, minimum solidified waste, and reduced radioactive discharge. Special challenges include kinetics, robustness to $\mathrm{pH}$ extremes typical of reprocessing streams, and ease of stripping for materials meant to be recycled. Many ion exchangers have been investigated, most of them metal oxide materials with layered and $3 \mathrm{D}$ structures such as zeolites, clays, and various silicates, titanates,

\section{Core Idea}

Improvements in ion exchange selectivity and capacity will open the door for broader use of these materials in the nuclear fuel cycle, especially in polishing operations leading to very high decontamination factors. zirconium phosphates, and niobates (Chitra et al. 2011; Hanna et al. 2020; Heirner et al. 2019; Alby et al. 2018). Some of these cases show low selectivity and do not remediate solutions with low residual concentrations to acceptable levels. Since high concentration of other ions are always present, the selective uptake of radioactive ions from nuclear waste still presents a challenge. Therefore, it is important to develop more effective and resilient ion exchangers with high efficiency, selectivity, and capacity for removal of radioactive ions from the complex wastewater for waste partitioning and contaminant remediation purposes. The separation science of inorganic ion exchangers revolves around the molecular understanding of various mechanisms such as electrostatic attraction, hydrogen bonding, and coordination chemistry. One key aspect to all such materials must be highest stability to extreme $\mathrm{pH}$ levels as well as to radiation, the latter being aggravated by the sustained high radiation doses borne by materials loaded with radionuclides.

Additional considerations include recyclability and reusability, as well as the capability of the materials to be converted to stable and final waste forms (zur Loye et al. 2018). The pore molecular structure and the nature of the atoms defining the pore are important for designing effective ion exchangers. Therefore, structure-property relationships at the molecular level of promising new classes of materials are required for further advancements. Ions of interest are $\mathrm{UO}_{2}{ }^{2+}, \mathrm{Co}^{2+}, \mathrm{Ni}^{2+}, \mathrm{Np}^{\mathrm{n}}, \mathrm{Am}^{2 / 3+}, \mathrm{AmO}_{2}{ }^{2 / 1+}, \mathrm{Pu}^{\mathrm{n}+},{ }^{99} \mathrm{Mo},{ }^{137} \mathrm{Cs}^{+}$, and ${ }^{90} \mathrm{Sr}^{2+}$, among others. Recently, metal sulfide ion exchangers have proven promising as a class of stable materials that can achieve uniquely high performance in removing radioactive ions such as uranyl (Figure 6.3) (Manos and Kanatzidis 2012; Sarma et al. 2016). Because of the soft Lewis base character of sulfide atoms, the metal sulfides offer bonding selectivity by coordinating to softer Lewis acidic metal ions (Manos and Kanatzidis 2016). The sulfides also remove Tc via their ability to reduce anions such as $\left[\mathrm{TcO}_{4}\right]^{-}$ (Neeway et al. 2016). Hybrid organic-inorganic materials are also envisioned and designed to have high 
surface areas and show very strong cation affinity. They can be synthetically tailored using principles of coordination chemistry to include many positively charged ions and to be selective toward tri- and tetravalent ions. Research is needed to identify the adsorption process in the novel materials using model tools such as synchrotron and neutron scattering and XANES (X-ray absorption near-edge structure) spectroscopy, which will provide deep understanding of mechanisms of ion removal from radioactive water.

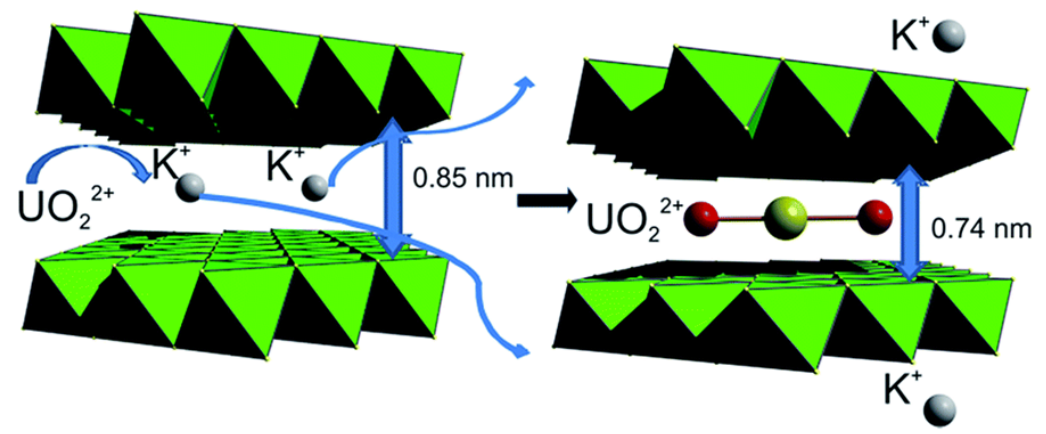

Figure 6.3. Ion exchange of uranyl adsorption by metal sulfide KMS-1 material. (Courtesy of INL.)

\subsubsection{Ion Exchangers on Polymer Supports and Composites}

Ion exchange resins have been known for decades. Their evolution into ion-selective polymers presents a challenge and an opportunity for developing novel systems for the treatment of nuclear waste. A significant advantage of polymer-based materials is that they can be designed to be both cation and anion ionexchangers. The latter is relevant because several radionuclides of interest are anionic in nature $\left({ }^{99} \mathrm{MoO}_{4}{ }^{2-}\right.$ and ${ }^{99} \mathrm{TcO}_{4}^{-}$) The concept of immobilizing selective ligands onto cross-linked polymer supports takes the development of ion-exchange resins to the next level and is a fertile area for research (Alexandratos 2018). Immobilizing custom ion-binding ligands onto cross-linked polymer supports is attractive for developing a more effective generation of materials with high capacity and chemical selectivity. Several categories of polymers can be defined based on key characteristics such as electrostatic, steric, geometric and electronic factors and ligands that attract and bind transuranic and related ions. Like the inorganic ion-exchange materials, to make the polymer-based composites field-deployable, significant challenges must be overcome including extreme $\mathrm{pH}$, capacity, selectivity in a multitude of competing ions, robustness to ionicstrength swing, kinetics, and understanding the effects of hydrophilic/hydrophobic interactions, hydration energies, and polymer crosslink level. Radiation stability stands out as a special challenge.

Polymeric sorbent materials can be prepared via high internal-phase emulsion (HIPE) polymerizations, and the polymeric foam backbone can be functionalized with ion-selective ligands targeting the radionuclides of interest. Development of separation columns based on polyHIPE foam materials could evolve into custom systems for the removal of radioactive ions. The polyHIPE foam materials have the advantage of relying on convective mass transport versus diffusive promising faster loading and sharper elution profiles. The rational design of each characteristic will require identifying the relationships between these factors and applying principles derived from new insights to tackle the inherent complex science will be an important focus of future research (Dzhardimalieva et al. 2018; Kim et al. 2018; Li et al. 2018). 


\subsubsection{Membranes}

Improved membrane systems and other related engineered forms offer opportunities (Wang et al. 2021; Yin et al. 2020). Porous membranes composed of effective ion-exchange materials and organic polymers could selectively transport metals with ease of operation and high throughput. Achieving this goal will entail overcoming challenges in gaining high selectivity while

\section{Core Idea}

Improvements in membrane design and structure will offer the possibility for broader use of these materials in the nuclear fuel cycle.

maximizing transport kinetics. Grafting of polymer chains with brush-like structures functionalized for specific separations performance onto polymer trunks with desired mechanical and chemical properties could yield increased numbers of active sites per unit of surface area compared to traditional crosslinked polymer matrices (Apel et al. 2019; Eom et al. 2021; Wang and Zhuang 2019). The combination of highcapacity organic and inorganic sorbents configured as membranes in cartridge-type systems could translate technologies being developed for selective extraction of heavy metal ions from polluted water to extracting $f$-elements from UNF reprocessing streams. In membranes composed of polymers, unexpected grafting/degrafting and carbon-chain cleavage induced by radiation may change the local structure and result in severe degradation in performance. Designing and testing for radiation stability of membranes is therefore important.

\subsubsection{Radiation Effects in Actinide Coordination Chemistry}

\section{Core Idea}

Integral consideration of radiation effects in the design of new ligands will shorten the timeline to deployment of new extractants, and could lead to systems in which radiolysis is beneficial to the separation process rather than detrimental.
An additional research challenge and priority for the development of advanced separation technologies is understanding the consequences of ionizing radiation on coordination chemistry, and our ability to predict and manage these effects, which are often detrimental to process performance (see Chapter 9). In both liquidliquid or solid-liquid separative technologies, radiation fields induce destruction of active compounds (ligands, phase modifiers, holdback agents, etc.) and materials (sorbents, ion exchangers, membranes, etc.) critical for the coordination and efficient separation of actinide and FP elements. In addition to this primary effect, the resulting degradation products often lead to unwanted secondary effects: reduced separation efficiency, changed physical properties of solvent mixtures, lower solvent-recycle longevity, CRUD formation, and other unexpected outcomes that impact the efficient recovery of valuable materials and the volume of hazardous radioactive waste for final disposal (Mincher et al. 2009a; Mincher et al. 2009b; Mincher et al. 2009c). Consequently, understanding this combination of radiation-induced active compound destruction and formation of non-bystander degradation products is critical for the (1) cost-effective development and innovation of separation technologies; (2) design and implementation of predictive radiation chemical models for process monitoring and longevity; and (3) potential ability to exploit radiolytic phenomenon to our benefit (e.g., strategic radiolysis of active species [ligands and additives]) to liberate specific degradation products that aid subsequent process stages.

To date, radiation chemistry studies in this area have focused on empirical investigations of the radiolytic robustness of active compounds (Mincher et al. 2009a; Mincher et al. 2009b; Mincher et al. 2009c), which have been useful for evaluating their suitability for proposed separative technology formulations from a stop-go perspective. However, the information gleaned so far is insufficient for the development of computer modeling capabilities for the prediction of (1) radiation-induced changes in separation efficiency and physical properties of solvent mixtures; (2) solvent-recycle longevity; and (3) molecular architectures suitable for radiation environments. This predictive knowledge gap has been exacerbated by the scarcity of 
radiation studies that have investigated the effect of metal ion complexation on the radiolytic behavior of active compounds, which is essential for their deployment in this area.

\subsection{Summary}

Aqueous-based methods for separation and purification of metal ions predominantly evoke the principles of the coordination chemistry. The thermodynamic variances resulting from ligand-induced dehydration and the formation of metal complexes offer a panorama of separative strategies. Select few options are applicable to the isolation of actinides from UNF, which requires the coordination reagents to withstand the relentless onslaught of hydrolytic and radiolytic damage. The most preferred options for actinide recovery either capitalize on the diverse redox chemistry of the early actinides or $5 f$ electron delocalization, relative to $4 f$, when formulating coordination environments applied on liquid-liquid or solid-liquid platforms. The established methods for actinide recycling at the back end of nuclear fuel cycle have resulted from decades of R\&D studies and clearly highlight six important features of a promising coordination reagent. The compatibility, selectivity, affinity, kinetics, stability, and predictability are inherent ligand properties that require consideration to simplify and compact actinide separations. Advances in coordination chemistry are needed to (1) improve the understanding of $f$-element selectivity, (2) accelerate ligand design, (3) manage FPs, (4) predict reagent stability, and ultimately (5) simplify the overall process. Theoretical descriptions of actinide binding can benefit from a data science approach that is supported by experimental ligand design efforts. New approaches to intelligent ligand design and chemical separations may combine ML tools, theoretical modeling, and high-throughput synthesis and screening to accelerate sampling of vast areas of chemical space. The coordination chemistries of actinides and $\mathrm{Ru}, \mathrm{Zr}, \mathrm{Nb}$, I, and $\mathrm{Tc}$ in the nuclear fuel cycle are uniquely linked, reinforcing the research needs in the area of FP control in actinide separations. Similarly, the coordination chemistry of actinides is inherently accompanied by the damaging presence of ionizing radiation. Collection of fundamental radiolytic parameters for molecular building blocks and reagent classes can be fundamentally transformed by using a data-science approach, computations, and high-throughput synthesis and screening. Advancements in all realms of nuclear fuel cycle science must ultimately lead to improved efficiency, robustness, and safety that will simplify and diversify the monoand multirecycling options for UNF.

\subsection{References}

Alby, D.; Charnay, C.; Heran, M.; Prelot, B.; Zajac, J. Recent Developments in Nanostructured Inorganic Materials for Sorption of Cesium And Strontium: Synthesis and Shaping, Sorption Capacity, Mechanisms, and Selectivity-A review. Journal of Hazardous Materials 2018, 344, 511-530.

Alexandratos, S. D. From Ion Exchange Resins to Polymer-Supported Reagents: An Evolution of Critical Variables. Journal of Chemical Technology and Biotechnology 2018, 93(1), 20-27.

Apel, P. Y.; Bobreshova, O. V.; Volkov, A. V.; Volkov, V. V.; Nikonenko, V. V.; Stenina, I. A.; Filippov, A. N.; Yampolskii, Y. P.; Yaroslavtsev, A. B. Prospects of Membrane Science Development. Membranes and Membrane Technologies 2019, 1(2), 45-63.

Authen, T. L.; Adnet, J.-M.; Bourg, S.; Carrott, M.; Ekberg, C.; Galán, H.; Geist, A.; Guilbaud, P.; Miguirditchian, M.; Modolo, G.; Rhodes, C.; Wilden, A. An Overview of Solvent Extraction Processes Developed in Europe for Advanced Fuel Recycling, Part 2-Homogeneous Recycling. Sep. Sci. Technol. 2021. https://doi.org/10.1080/01496395.2021.2001531

Chitra, S.; Viswanathan, S.; Rao, S. V. S.; Sinha, P. K. Uptake of Cesium and Strontium by Crystalline Silicotitanates from Radioactive Wastes. Journal of Radioanalytical and Nuclear Chemistry 2011, 287(3), 955-60. 
Choppin, G. R.; Jensen, M. P. Actinides in Solution: Complexation and Kinetics, in L. R. Morss, N. M. Edelstein, J. Fuger (Eds.), The Chemistry of the Actinide and Transactinide Elements, vol. 4, Springer, Dordrecht, the Netherlands, pp. 2524-21, Chapter 23 (2006).

Dzhardimalieva, G. I.; Uflyand, I. E. Design Strategies of Metal Complexes Based on Chelating Polymer Ligands and Their Application in Nanomaterials Science. Journal of Inorganic and Organometallic Polymers and Materials 2018, 28(4), 1305-93.

Eom, H. H.; Kim, Y.; Harbottle, D.; Lee, J. W. Immobilization of KTS-3 on an Electrospun Fiber Membrane for Efficient Removal of $\mathrm{Cs}^{+}$and $\mathrm{Sr}^{2+}$. Journal of Environmental Chemical Engineering 2021, 9(5), 105991.

Geist, A.; Panak, P. J. Recent Progress in Trivalent Actinide and Lanthanide Solvent Extraction and Coordination Chemistry with Triazinylpyridine N Donor Ligands. Solvent Extraction Ion Exchange 2021, 39, 128-51.

Hanna, S. L.; Rademacher, D. X.; Hanson, D. J.; Islamoglu, T.; Olszewski, A. K.; Nenoff, T. M.; Farha, O. K. Structural Features of Zirconium-Based Metal-Organic Frameworks Affecting Radiolytic Stability. Industrial \& Engineering Chemistry Research 2020, 59(16), 7520-26.

Heirner, B. W.; Paap, S. M.; Sasan, K.; Brady, P. V.; Nenoff, T. M. 110th Anniversary: Industrial Process Water Treatment and Reuse Enabled by Selective Ion Exchange Materials. Industrial \& Engineering Chemistry Research 2019, 58(32), 14873-79.

Hua, Y.; Flood, A. H. Flipping the Switch on Chloride Concentrations with a Light-Active Foldamer. Journal of the American Chemical Society 2010, 132(37), 12838-40.

Kaltsoyannis, N.; Hay, P. J.; Li, J.; Blaudeau, J.-P.; Bursten, B. E. Theoretical Studies of the Electronic Structure of Compounds of the Actinide Elements, in L. R. Morss, N. M. Edelstein, J. Fuger (Eds.), The Chemistry of the Actinide and Transactinide Elements, vol. 3, Springer, Dordrecht, the Netherlands, pp. 1893-2012, Chapter 17, 2006.

Kim, Y.; Kim, I.; Lee, T. S.; Lee, E.; Lee, K. J. Porous Hydrogel Containing Prussian Blue Nanoparticles for Effective Cesium Ion Adsorption in Aqueous Media. Journal of Industrial and Engineering Chemistry 2018, 60, 465-74.

Li, J.; Dai, X.; Zhu, L.; Xu, C.; Zhang, D.; Silver, M. A.; Li, P.; Chen, L. H.; Li, Y. Z.; Zuo, D. W.; Zhang, H.; Xiao, C. L.; Chen, J.; Diwu, J.; Farha, O. K.; Albrecht-Schmitt, T. E.; Chai, Z. F.; Wang, S. A. $\left(\mathrm{TcO}_{4}^{-}\right)-\mathrm{Tc}-99$ Remediation by a Cationic Polymeric Network. Nature Communications 2018, 9, 3007.

Lumetta, G. J.; Rapko, B. M.; Hay, B. P.; Garza, P. A.; Hutchison, J. E.; Gilbertson, R. D., A novel bicyclic diamide with high binding affinity for trivalent f-block elements. Solvent Extr. Ion Exch. 2003, 21 (1), 29-39.

Manos, M. J.; Kanatzidis, M. G. Layered Metal Sulfides Capture Uranium from Seawater. Journal of the American Chemical Society 2012, 134(39), 16441-46.

Manos, M. J.; Kanatzidis, M. G. Metal Sulfide Ion Exchangers: Superior Sorbents for the Capture of Toxic and Nuclear Waste-Related Metal Ions. Chemical Science 2016, 7(8), 4804-24.

Mincher, B. J.; Modolo, G.; Mezyk, S. P. Review Article: The Effects of Radiation Chemistry on Solvent Extraction: 1. Conditions in Acidic Solution and a Review of TBP Radiolysis. Solvent Extraction and Ion Exchange 2009a, 27(1), 1-25. https://doi.org/10.1080/07366290802544767.

Mincher, B. J.; Modolo, G.; Mezyk, S. P. Review Article: The Effects of Radiation Chemistry on Solvent Extraction: 2. A Review of Fission-Product Extraction. Solvent Extraction and Ion Exchange 2009b, 27(3) 331-53. https://doi.org/10.1080/07366290902821263. 
Mincher, B. J.; Modolo, G.; Mezyk, S. P. Review Article: The Effects of Radiation Chemistry on Solvent Extraction 3: A Review of Actinide and Lanthanide Extraction. Solvent Extraction and Ion Exchange 2009c, 27(5-6) 579-606. https://doi.org/10.1080/07366290903114098.

Modolo, G.; Geist, A.; Miguirditchian, M. Minor Actinide Separations in the Reprocessing of Spent Nuclear Fuels: Recent Advances in Europe, in. Taylor (Ed.), Reprocessing and Recycling of Spent Nuclear Fuel, Woodhead Publishing, Elsevier, Cambridge, UK, pp. 245-87, Chapter 10, (2015).

Moyer, B. A.; Lumetta, G. J.; Mincher, B. J. Minor Actinide Separations in the Reprocessing of Spent Nuclear Fuels: Recent Advances in the United States, in: R. Taylor (Ed.), Reprocessing and Recycling of Spent Nuclear Fuel, Woodhead Publishing, Elsevier, Cambridge, UK, pp. 289-323, Chapter 11 (2015).

Moyer, B. A.; Custelcean, R.; Hay, B. P.; Sessler, J. L.; Bowman-James, K.; Day, V. W.; Kang, S. O. A Case for Molecular Recognition in Nuclear Separations: Sulfate Separation from Nuclear Wastes. Inorganic Chemistry 2013, 52, 3473-90.

Nash K. L., Madic, C.; Mathur, J. N.; Lacquement, J. Actinide Separation Science and Technology, in: L. R. Morss, N. M. Edelstein, J. Fuger (Eds.), The Chemistry of the Actinide and Transactinide Elements, vol. 4, Springer, Dordrecht, the Netherlands, pp. 2622-798, Chapter 24 (2006).

Neeway, J. J.; Asmussen, R. M.; Lawter, A. R.; Bowden, M. E.; Lukens, W. W.; Sarma, D.; Riley, B. J.; Kanatzidis, M. G.; Qafoku, N. P. Removal of $\mathrm{TcO}_{4}^{-}$from Representative Nuclear Waste Streams with Layered Potassium Metal Sulfide Materials. Chemistry of Materials 2016, 28(11), 39763983.

Poinssot, Ch.; Boullis, B.; Bourg, S. Role of Recycling in Advanced Nuclear Fuel Cycles, in R. Taylor (Ed.), Reprocessing and Recycling of Spent Nuclear Fuel, Woodhead Publishing, Elsevier, Cambridge, UK, pp. 27-48, Chapter 2 (2015).

Sarma, D.; Malliakas, C. D.; Subrahmanyam, K. S.; Islama, S. M.; Kanatzidis, M. G. $\mathrm{K}_{2 x} \mathrm{Sn}_{4-x} \mathrm{~S}_{8-x}(\mathrm{x}=$ 0.65 - 1): A New Metal Sulfide for Rapid and Selective Removal of $\mathrm{Cs}^{+}, \mathrm{Sr}^{2+}$ and $\mathrm{UO}_{2}{ }^{2+}$ ions. Chemical Science 2016, 7(2), 1121-32.

Sessler, J. L.; Gale, P. A.; Cho, W. S. Introduction. In Anion Receptor Chemistry; Stoddart, J. F., Ed.; Royal Society of Chemistry: London, 2006; pp 1-26.

Shen, N.; Yang, Z.; Liu, S. et al. ${ }^{99} \mathrm{TcO}_{4}^{-}$Removal from Legacy Defense Nuclear Waste by an AlkalineStable 2D Cationic Metal Organic Framework. Nature Communication 2020, 11, 5571. https://doi.org/10.1038/s41467-020-19374-9.

Su, J.; Batista, E. R.; Boland, K. S.; Bone, S. E.; Bradley, J. A.; Cary, S. K.; Clark, D. L.; Conradson, S. D.; Ditter, A. S.; Kaltsoyannis, N.; Keith, J. M.; Kerridge, A.; Kozimor, S. A.; Löble, M. W.; Martin, R. L.; Minasian, S. G.; Mocko, V.; La Pierre, H. S.; Seidler, G. T.; Shuh, D. K.; Wilkerson, M. P.; Wolfsberg, L. E.; Yang, P. Energy-Degeneracy-Driven Covalency in Actinide Bonding, Journal of the American Chemical Society 2018, 140, 17977-84. https://doi.org/10.1021/jacs.8b09436.

Thevenet, A.; Miljkovic, A.; La Cognata, S.; Marie, C.; Tamain, C.; Boubals, N.; Mangano, C.; Amendola, V.; Guilbaud, P. Syntheses and Evaluation of New Hydrophilic Azacryptands Used as Masking Agents of Technetium in Solvent Extraction Processes. Dalton Transactions 2021, 50.

Wang, Y. L.; Long, J.; Xu, W. J.; Luo, H.; Liu, J.; Zhang, Y. P.; Li, J. C.; Luo, X. G. Removal of Uranium(VI) from Simulated Wastewater by a Novel Porous Membrane Based on Crosslinked Chitosan, UiO-66- $\mathrm{NH}_{2}$ and Polyvinyl Alcohol. Journal of Radioanalytical and Nuclear Chemistry 2021, 328(1), 397-410. 
Wang, J.; Zhuang, S. Removal of Cesium Ions from Aqueous Solutions using Various Separation Technologies. Reviews in Environmental Science and Bio/Technology 2019, 18(2), 231-269.

Yin, L.; Hu, B. W.; Zhuang, L.; Fu, D.; Li, J. X.; Hayat, T.; Alsaedi, A.; Wang, X. K. Synthesis of Flexible Cross-Linked Cryptomelane-Type Manganese Oxide Nanowire Membranes and their Application for U(VI) and Eu(III) Elimination from Solutions. Chemical Engineering Journal 2020,381 .

zur Loye, H. C.; Besmann, T.; Amoroso, J.; Brinkman, K.; Grandjean, A.; Henager, C. H.; Hu, S. Y.; Misture, S. T.; Phillpot, S. R.; Shustova, N. B.; Wang, H.; Koch, R. J.; Morrison, G.; Dolgopollova, E. Hierarchical Materials as Tailored Nuclear Waste Forms: A Perspective. Chemistry of Materials 2018, 30 (14), 4475-88. 


\section{FRD 6: Develop Technologies for Real-Time Quantification of Chemical Species}

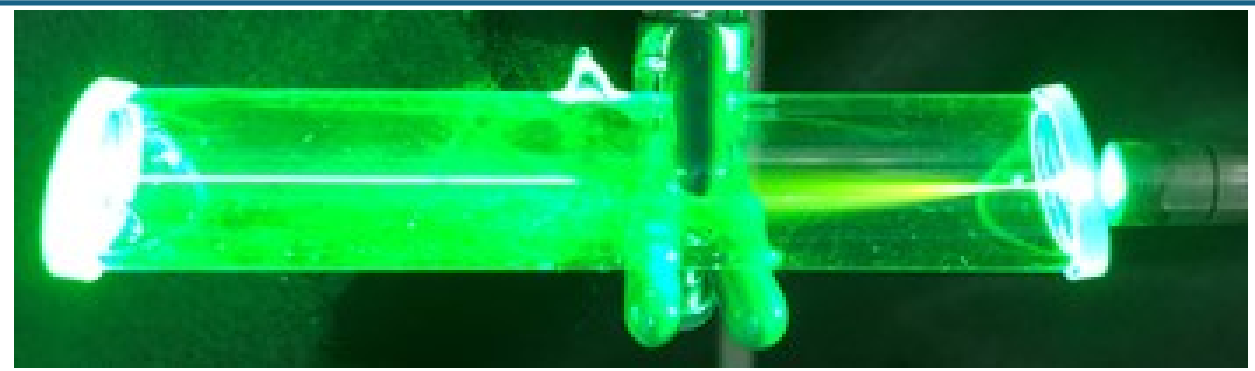

Raman spectroscopic analysis of an iodine-containing gas. (Courtesy of PNNL.)

Economics and safeguarding of special nuclear materials (SNM) are two significant barriers This FRD describes methods to enable real-time or near real-time quantification of critical constituents in nuclear recycling streams. Multiple techniques are desired for an advanced plant, as each generally has limitations and provides unique information. Combinations of techniques provide enhanced reliability, utility, and safeguarding of SNM. Analytical methods that would benefit from research include optical spectroscopy (absorbance and Raman), electrochemical sensors, laser-induced breakdown spectroscopy, laser ablation molecular isotope spectrometry, X-ray spectroscopy techniques, and sensors for gas-phase species. Advancements in instrumentation and data analysis techniques (e.g., chemometric modeling) will push these technologies forward to enable more rapid process development and eventual deployment in processing plants for the purposes of process monitoring and control, as well as safeguarding of special nuclear materials (SNMs). Research needs for the related monitoring requirements for solution volumes, densities, and flowrates are also identified.

\subsection{Introduction}

Economics and nuclear proliferation are the most often cited barriers to deployment of nuclear fuel recycling. Capital costs are directly related to the size of the facility, which in turn is tied to the throughput rate and delays to track the mass balance of special nuclear material (SNM). Furthermore, sampling and analysis (both for safeguards and process control) contribute to the operating costs of the recycling facility. A third significant barrier to deployment is the decades-long development time required. Shortening of this development period would make investment in nuclear fuel recycling more attractive.

Integration of process monitoring, the in situ and real-time analysis of a chemical system, could address challenges in five major areas:

1. Enabling foundational characterization of chemical systems or processes: Drastically reducing time to devise advanced separation schemes

2. Accelerating technology development by enabling efficient and informed process demonstrations: Significantly reducing time to develop, demonstrate, and optimize processes at the pilot scale

3. Allowing for cost effective and safe deployment: Reducing capital costs associated with construction, management, and operations

4. Modernizing safeguards regimes: Deploying technologies for real-time tracking of SNM

5. Minimizing and managing process upsets: Enabling automated process control that reduces down time and speeds up recovery 
A wide variety of process-monitoring tools can be leveraged within the nuclear separations field to provide a range of process data. Of specific interest are tools that can provide insight into chemical composition including optical spectroscopy, electroanalytical techniques, laser-induced breakdown spectroscopy (LIBS), X-ray spectroscopic techniques, and gas-phase sensors. Progress in translating these techniques into real-time process monitoring tools will rely on progress in the crosscutting areas discussed in Chapter 9. For example, chemometric modeling is a form of ML that can take data input from multiple sources and translate them into compositional information for complex multicomponent processing streams; modeling and simulation is critical to the correct interpretation of X-ray spectroscopic data; and understanding radiation effects on equipment and materials (e.g., spectroscopy probes and fiber optic cables) is required for deployment of these analytical systems in radiochemical processing environments.

In addition to applying real-time analytical chemistry techniques, methods to track mass flows of materials are required to quantitatively track material (especially SNM) in a plant setting. Bubbler technology is traditionally deployed for this purpose, but improved innovative methods to perform these functions are needed.

\subsection{Technical Challenges and Future Directions}

\subsubsection{Advancing Optical Spectroscopy}

\section{Core Idea}

Machine learning tools will advance optical spectroscopic methods for real-time monitoring of nuclear processing streams - accelerating process development, revolutionizing plant process and control systems, and modernizing safeguards regimes.
Optical spectroscopy is a powerful option because it can provide identification and quantification of target analytes while also enabling characterization of oxidation states and speciation (Lines et al. 2017, 2020a). It can also add robustness to changing conditions and the ability to detect and diagnose in realtime off-normal behavior. The primary challenge of this approach is gaining selectivity for target analytes in complex samples or processing streams with complex spectral signatures such as those illustrated in Figure 7.1. However, advances in data-analysis methods have enabled application of optical techniques to increasingly complex chemical systems. Specifically, ML tools such as chemometric analysis can provide an avenue to enhance selectivity and improve quality of characterization information (Lines et al. 2020a; Clifford et al. 2021).

Integration of optical techniques can provide in situ, real-time insight into process or sample chemistry. This powerful information can open new and impactful routes to advance, optimize, and deploy nuclear materials processing schemes. Overall, methods are needed to (1) accelerate flowsheet development, (2) accurately measure amounts of SNM in the various process streams in real time in an industrial nuclear fuel recycling plant, and (3) automate process control to achieve maximum process efficiency with minimal operator intervention. Optical techniques are ideal to meet these goals because they can provide knowledge about the role and impact of transient or short-lived species in separation processes, information on the chemical speciation of important fuel components under process conditions (Heller et al. 2017), and the influence of process conditions on the flow of these components through the system. To meet these needs, the following areas represent key R\&D opportunities across a range of processing systems. 

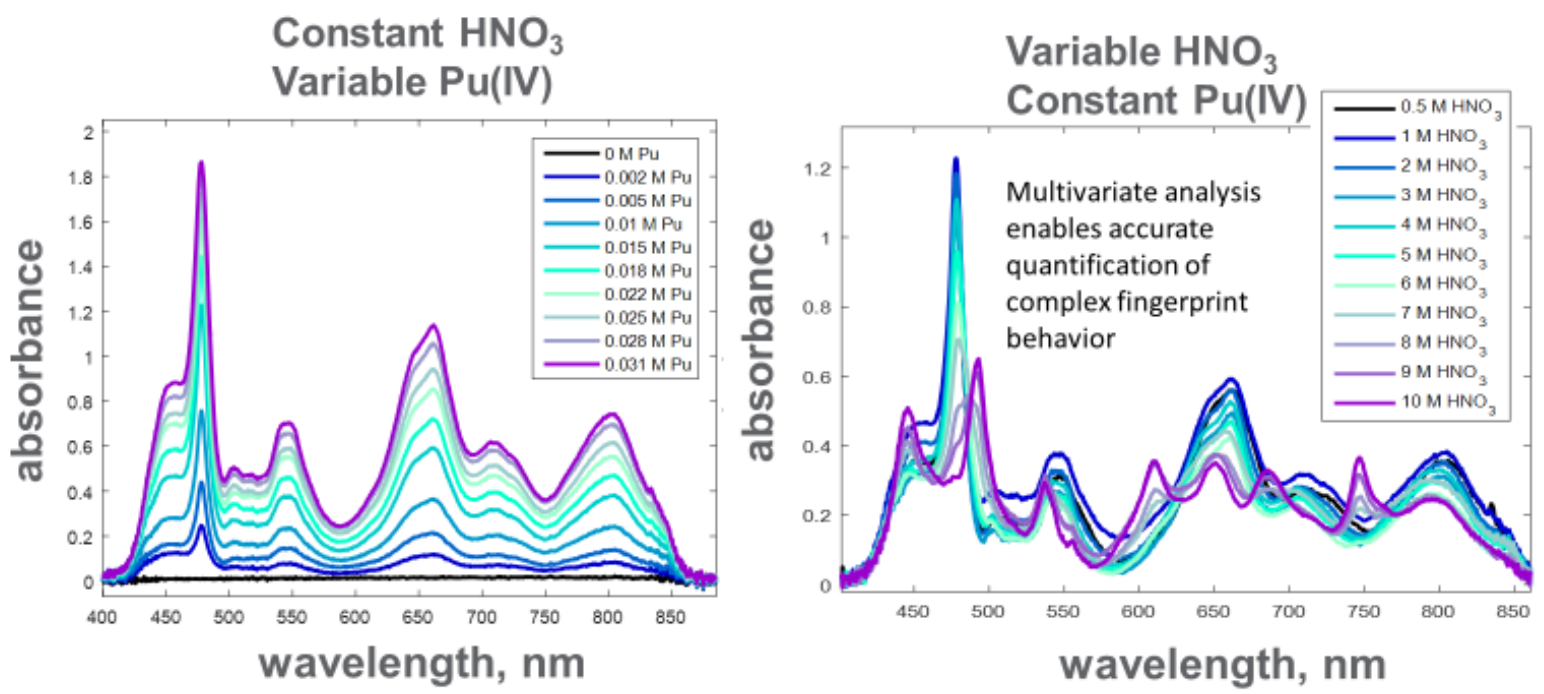

Figure 7.1. Experimental observation of spectra from a variety of process conditions (Lines 2017, reprinted with permission from American Chemical Society).

\section{Aqueous systems}

Using optical monitoring techniques to characterize new separation mechanisms (e.g., novel extractants) can drastically enhance the speed of process development. Specifically, exploring the simultaneous characterization of relevant solvent systems using multiple forms of optical spectroscopy, could provide enhanced understanding of process chemistry. In this case, a combination of electronic and vibration spectroscopy such as UV-vis absorbance and Raman could be highly valuable.

Recent demonstrations have indicated the utility of process monitoring to enable real-time control (Lumetta et al. 2019; Lines et al. 2020b). However, these demonstrations relied only on following concentrations of target analytes. This could be improved by identifying mechanisms to combine optical information (concentration) with flow sensor data to ultimately output mass-balance information on the process.

\section{Pyrochemical systems}

Because of its highly complex chemistry and the opportunity to enable advanced fuel processing and recycle, pyrochemical processing represents a key area in which the integration of optical process monitoring technology can support significant process development (Schroll et al. 2016). Utilizing optical monitoring (e.g., UV-vis absorbance or Raman spectroscopy) during bench-scale process development can enable drastic reductions in the time to deploy pyrochemical processes. This overcomes the major challenge of grab-sample analysis, which causes salt systems to experience drastic oxidation states and speciation variation upon freezing. Development of modular optical systems as well as chemometric models for realtime characterization would support these deployments.

\subsubsection{Developing Electrochemical Sensors}

Real-time quantification of the chemical species within fuel processing streams is essential for proper process control and nuclear material accountancy. Electroanalytical sensors offer a variety of approaches for achieving these measurements in aqueous, pyroprocessing, and molten salt-relevant applications. Electroanalytical sensors use the electrochemical response of electrodes immersed into the process solution 
to determine the chemical composition and redox state of the fluid. This electrochemical response can range from passive measurements (e.g., certain potentiometric approaches) to active signals applied to the electrodes (e.g., voltammetry). The concentration of species in the fluid is ultimately related to the sensor response using electroanalytical formulas or correlation curves.

\section{Core Idea}

New electrochemical sensor technologies will advance pyrochemical processing approaches to recycling of metallic fuels and cleanup and restoration of MSR fuels.
Electroanalytical measurements in radiological systems are challenging because of several factors. For aqueous process streams, the effects of radiation, impurities, electroactive ligands, and multicomponent solutions make quantitative measurements nontrivial. Extensive work for the electrochemical determination of An and Ln concentrations in these solutions has taken place (Schumacher et al. 2013). This work has included

studies using a variety of electrode systems including Hg-based electrodes, solid electrodes, carbon-paste electrodes, and microelectrodes. The electrochemical window of water typically limits the range of applied potentials, which can constrain the specific chemical species that can be measured. Most aqueous studies have concentrated on electroanalytical approaches to determine fundamental properties of the dissolved chemical species (e.g., kinetics values), but sensors for process monitoring have been pursued as well (Oleneva et al. 2019). Even with the research into fundamental properties, significant data gaps exist that can often preclude the use of electroanalytical formulas to quantitatively analyze aqueous solutions. Needed areas of research to enable the quantification of aqueous process streams include the development of sensors with increased selectivity, range, and longevity, as well as approaches for providing accurate measurements in complex multicomponent salts.

Electroanalytical sensors are also crucial for the monitoring of pyroprocessing and other molten saltrelevant activities. Extensive development of these types of sensors has been conducted toward that goal (Iizuka et al. 2001; Tylka et al. 2015; Fredrickson et al. 2019). Molten salts create additional challenges including high temperatures and corrosive conditions that are different from those encountered in aqueous systems. With careful selection of materials, long-duration monitoring lasting for years has been shown to be possible (Hoyt et al. 2021). Stable reference electrodes are a larger challenge for molten salts compared to aqueous situations; identification of suitable reference electrode capable of being used in a broad range of radiological salts is an ongoing challenge. Multicomponent salts, such as those encountered when dealing with spent fuel, are an additional challenge. Fundamental data gaps exist for a variety of key species in these salts. Methods, techniques, and materials for quantitative measurements that can work across a wide range of concentrations are essential.

In total, the development of electrochemical approaches will likely provide notable enhancement to fuel cycle operations. Alongside experiments to close existing data gaps for fundamental properties, approaches to achieve suitable selectivity, stability, and longevity will be important to ensure that fuel processing systems that make use of aqueous solutions or molten salts can be properly operated and safeguarded.

\subsubsection{Applying LIBS and Laser Ablation Molecular Isotope Spectrometry (LAMIS) for Real-time Measurement}

In recent decades, LIBS has been developed, providing the potential for in situ and real-time measurements for nuclear applications. In LIBS, a short laser pulse is focused onto a sample, resulting in a hightemperature plasma as shown in Figure 7.2. Emitted light from the plasma is collected through a gated detector (specific time periods after plasma formation), where atoms return to ground states with the emission of characteristic photons. This collected light is used to generate a unique spectrum of the chemical elements in the sample. From the spectrum, qualitative data can be determined from the wavelengths of the spectral peaks, and quantitative data can be determined from the peak intensities. LIBS analysis can be 
performed in different material forms (liquid, gas, solid, aerosol) and can be applied remotely if an optical path to the in situ sample is available (light pipe, fiber optics, open air, etc.).

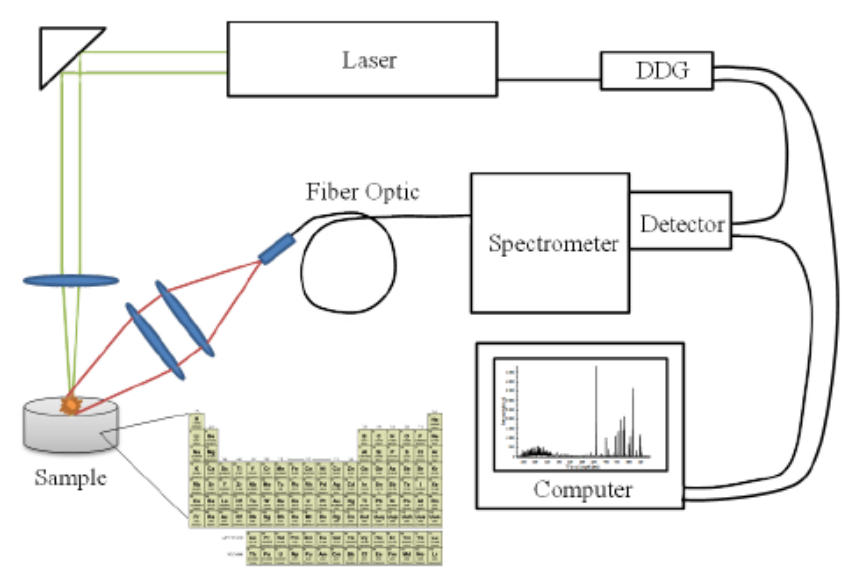

Figure 7.2. Layout of a typical LIBS setup (Williams 2016).

The first demonstration of LIBS to measure nuclear material occurred when Watcher and Cremers (1987) successfully measured the concentration of $U$ in nitric acid. Many other successful LIBS studies have been conducted over the years measuring nuclear materials (Singh et al. 1996; Fichet et al. 1999; Sarkar et al. 2008; Emmert et al. 2011; Wu et al. 2020). However, challenges exist when performing LIBS with heavy elements like the actinides; notably, the spectra exhibit interferences (peaks close together and overlapping) due to the density of spectral lines. Additionally, samples containing more elements such as fission and corrosion products would result in more complex spectra with significant spectral overlap. These interferences are problematic because the traditional univariate analysis approach relied on selecting a single representative peak for the element of interest without an interference to develop a calibration curve.

Using more advanced high-resolution spectrometers, the ability to resolve spectral lines increases. However, spectral interferences in more complicated samples continue to limit the capability of LIBS. Fortunately, in recent years, advanced calibration approaches including partial least-squares regression and principalcomponent analysis became more commonplace (Andrade et al. 2010). As described above for the optical spectroscopy systems, in these chemometric

\section{Core Idea}

In situ application of LIBS and LAMIS can provide a new tool for real-time monitoring of nuclear processing streams for application to plant process and control systems, and safeguards. approaches, the entire spectrum is analyzed to identify principal components that can be used to generate calibration models. Since the entire spectrum can be used, the models are less sensitive to spectral interferences and are more robust in complex material matrices. Utilization of LIBS and chemometric analysis has been successfully applied to a complex yellowcake sample for which the geographical origin of the material was determined (Sirven et al. 2009). Unfortunately, the true potential of LIBS coupled with chemometrics has yet to be tested in more complex sample matrixes such as real UNF and in reprocessing streams.

LIBS has also been studied as a method to measure the concentrations of different isotopes in nuclear materials. Barefield et al. (2013) were able to determine isotopic contents from a sample of MOX fuel using LIBS with a high-resolution spectrometer. However, the isotopic shifts in the wavelengths are small, and isotopic analysis using LIBS is not practical for most applications. A recent analytical approach using a LIBS setup called laser ablation molecular isotope spectrometry (LAMIS) was used to more effectively 
determine elemental isotopes (Bol'shakov et al. 2016). In practice, LIBS and LAMIS are the same with one small variation: in LAMIS, the detector is gated to collect light later in the plasma lifetime where molecular recombination is occurring. The isotopic shifts during molecular recombination are significantly larger, and thus measuring the isotopic shifts in nuclear materials is feasible even without high-resolution spectrometers.

Work done using LIBS and LAMIS in the nuclear industry has focused primarily on the feasibility of the techniques for nuclear applications. Applying the LIBS and LAMIS techniques at an engineering scale is needed to move the technology closer to application in nuclear separations. Most applications require functionality in harsh radiological conditions, high temperatures, and remote operation. Some of the concerns these conditions pose are optical paths and potential degradation of optics, optics alignment, and sampling access into the process streams. Solutions to some of these issues have been discussed in the literature (Wu et al. 2020), but the next steps toward application have not been taken.

\subsubsection{Applying X-ray Spectroscopies to Characterize Nuclear Processing Systems}

Development of the next generation of nuclear reactors including MSRs necessitates an understanding of the chemical and physicochemical properties of the fissile fuel constituents and fission/activation products. The elemental and chemical diversity of these materials often exists in different physical states and presents significant challenges in simultaneously probing and quantifying them. These challenges necessitate finding advanced techniques that allow monitoring of the complex chemical processes that take place in fuel cycle separations. Using a method that relies on the atomic structure of elements rather than presence of specific chromophores would be highly beneficial.

X-ray absorption spectroscopy (XAS) is uniquely suited for probing individual elements in heterogeneous multielement systems such as those related to the nuclear fuel process streams, providing information on oxidation state, chemical bond coordination, and quantitative elemental concentration and spatial distribution. This technique is particularly attractive because it can be applied to both solid (crystalline and amorphous) and liquid materials. Among other methodologies, XAS stands out as very sensitive to the local electronic properties and bonding environments of the metal atoms and therefore can monitor structural variations in operando conditions. XAS facilitates further development of the theory by comparing predictions with experimental data, enabling improved data interpretation and analysis.

XAS spectra contain two regions with complimentary information. X-ray absorption near-edge structure (XANES) corresponds to lower excitation energies and is defined by electronic transitions of absorbing atoms revealing information about their electronic state, local symmetry, and chemical bonding. The XANES spectral region is followed by the extended X-ray absorption fine structure (EXAFS), which provides information about the spatial arrangement and distribution of nearest neighbors surrounding the interrogated atoms. EXAFS is sensitive to structural disorders and is therefore affected by the reaction conditions, such as high temperatures, but XANES spectral features retain high signal-to-noise ratio under the wide range of experimental conditions, including in samples with low concentrations of the interrogated Core Idea
Development of laboratory X-ray
absorption/emission instrumentation,
measurement methodologies, and DFT- and
ML-assisted data interpretation tools will enable
in situ measurements of fuel constituents and
fission products, enhancing our understanding
of their behavior under operando conditions
and informing theoretical modeling and
accelerating technology development. atoms.

XAS measurements typically require a synchrotron X-ray photon source, and several beamlines over the world have been dedicated for the examination of radioactive materials. Availability of these dedicated 
beamlines has streamlined investigations of actinides and radioactive fission products (e.g., Tc) and recently facilitated in situ studies of the molten salt systems at relevant temperature regimes (Smith et al. 2019), proving the beamlines to be highly beneficial for expanding fundamental knowledge and for acceleration of technological innovation. Development of instrumentation and corresponding methods adapted for use in a laboratory or plant setting for analysis of fuel cycle process samples is a critical need for future research. Recently, there has been a significant progress in design of such systems for various research and analytical purposes (Ditter et al. 2020; Jahrman et al. 2019), which are now commercially available (e.g., see EasyXAFS 2022).

In the recent characterization study, a laboratory-scale XAS technique was used to investigate chloride salts of trivalent lanthanides including Eu, Gd, Tb, and Dy; DFT and time-dependent DFT computations were performed to obtain ground-state structures, lattice parameters, and to simulate $\mathrm{L}_{3}$-edge XANES spectra (Boglaienko et al. 2022). The obtained spectral data were critically compared with the synchrotron results, finding a good agreement and validating performance of the laboratory spectrometer for the $f$-element measurements. Currently, this capability is being extended to incorporate a microfurnace compatible with transmission-mode XAS designed for the in situ high-temperature measurements of molten salt systems. This design has been tested with a laboratory-scale spectrometer, easyXAFS300, that was modified with a stationary detector and moving X-ray source (Mo anode) and equipped with a miniature vacuum furnace holding a sample pellet containing anhydrous $\mathrm{EuCl}_{3}$ with or without $\mathrm{KCl}$ on molten salts. The obtained XANES spectral overlay demonstrates in situ monitoring of Eu reduction-oxidation dependence on the matrix composition with temperature evolution and quantifies dynamic redox profiles during melting (Figure 7.3).
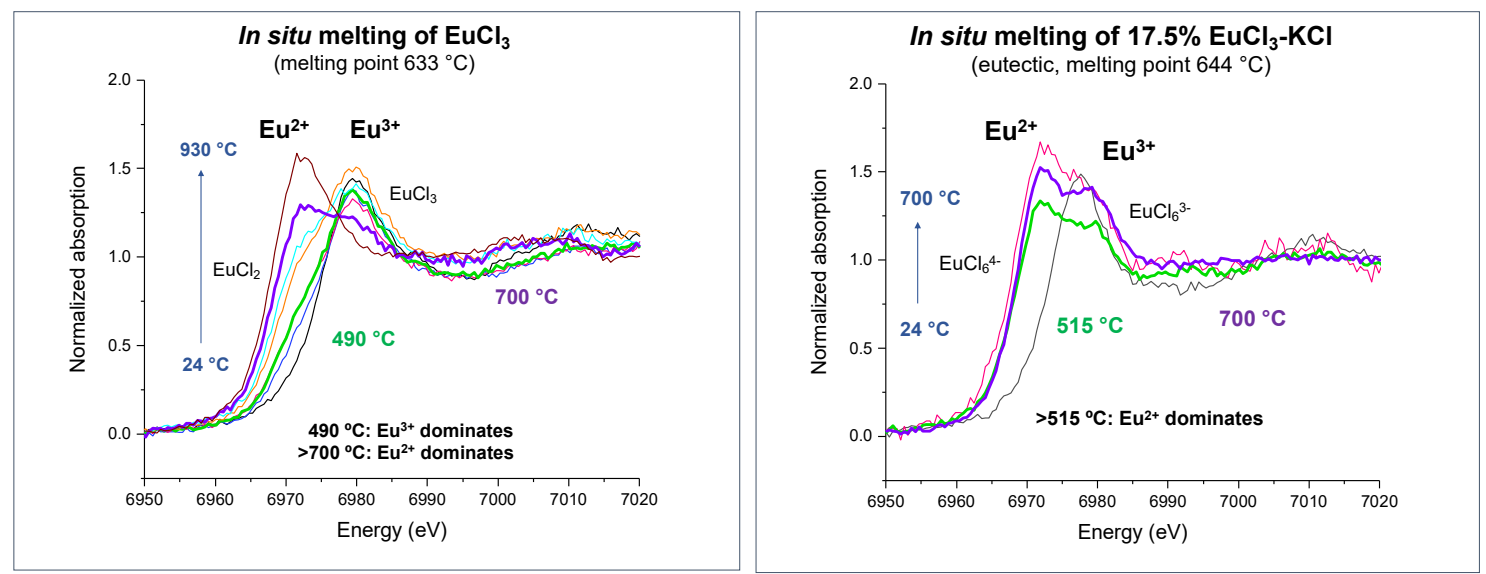

Figure 7.3. Temperature-dependent XANES spectral overlays (one scan per temperature point) of anhydrous $\mathrm{EuCl}_{3}$ (left) and $17.5 \mathrm{~mol} \% \mathrm{EuCl}_{3}-82.5 \mathrm{~mol} \% \mathrm{KCl}$ eutectic composition collected using easyXAFS300 equipped with a miniature vacuum furnace (Boglaienko et al. 2021).

This demonstration study highlights the need for the adaptation of the laboratory XAS/XES spectrometers for the in situ and in operando measurements, including design of dedicated sample holders that sustain controlled exposure of the interrogated material to the desired reaction environments such as complex composition of the reaction mixture, chemically aggressive solvent media, high temperature, and high radiation fields, without compromise to the quality of measurements. If developed, these setups would inform multifaceted behavior of the fuel constituents, in-growing fission products, and metal ions accumulating because of corrosion of the structural materials. The latter is of particular importance for the determination of the corrosivity of the molten salt media and can guide selection of the containment materials and melt composition. 
A nontrivial challenge associated with the interpretation of the XAS spectra lies in acquiring sufficient understanding of the fundamentals behind this technique and developing software tools to decode the spectral information. Quantitative analysis of the XAS spectra relies on the availability of reference spectra and therefore requires either building relevant databases for the elements of interest or generating them a priori using theory and integration of previously validated DFT models against experimental spectral information. Machine-learning methodologies with their ability to extract even minute and often hidden variations in experimental data can greatly benefit XAS data interpretation by not only simplifying it but also by potentially revealing new structural information and facilitating integration of the atomistic simulations (Timoshenko and Frenkel 2019).

\subsubsection{Developing Sensitive Sensors for Gas-Phase Species}

Sensors for sensitive and selective detection and measurement of gas-phase analytes are needed for monitoring and control of separation processes, accountability, environmental monitoring, and occupational safety in nuclear fuel recycling. Gas-phase analytes of concern include oxygen, hydrogen, moisture, noble gases, $\mathrm{NO}_{x}$, volatile organics, and volatile radionuclides. Most sensors rely on polymers or semiconductor films that adsorb or react with the gas-phase analytes to monitor the changes in mass, optical, electrical, mechanical, or radiometric properties of these films. Key challenges in designing the sensing materials primarily concern sensitivity and selectivity. Stability to extreme conditions and variable process streams represents and additional practical challenge. The magnitude of the sensor response depends on a large number of factors in addition to analyte concentration, including humidity, temperature, competing gas-phase species, and degradation. Understanding the underlying sensing mechanism at the molecular level as well as the effect of such interferences represents the key to sensor design to achieve the desired response. A generic design strategy is depicted in Figure 7.4. Robust materials designed for separation of gas-phase species with ideal properties as identified under FRD 3 provide an ideal starting point to adapt and integrate these advanced functional materials into new sensing devices.

New sensing materials and devices are needed for gas-phase sensors in fuel recycle applications. Although progress has been made broadly for many industrial applications, needs for nuclear separations remain generally unfulfilled. Over the past few years, advanced functional materials such as zeolites, porous organic cages, supramolecular hosts, MOFs, and COFs fashioned as thin films have been integrated into mechanical devices (surface acoustic wave, microcantilevers, etc.), electrodes, and optodes all of which convert chemical response into read-out signals. As an example for non-nuclear application, wireless surface acoustic-wave sensors coated with a selective sorbent layer enabled a novel sensor array for simultaneous detection of $\mathrm{CO}_{2}$ and $\mathrm{CH}_{4}$ at ambient conditions (Devkota et al. 2018). Similarly, Lustig et al. (2017) used luminescent metal-organic frameworks (LMOFs) as sensing materials. The change in the luminescence intensity of the LMOF can be used as a probe to recognize the presence of interacting species. Typically, intensity change is the usual mode of detection in case of LMOFs with enhancement or quenching being a by-product of the nature of interaction or electronic characteristics of the guest involved depending on the extent of interaction (electron and energy-transfer mechanisms) found to have a role in directing the change of luminescence intensity. Various LMOFs have been used in a range of applications for detection of biomolecules, environmental toxins, etc. 


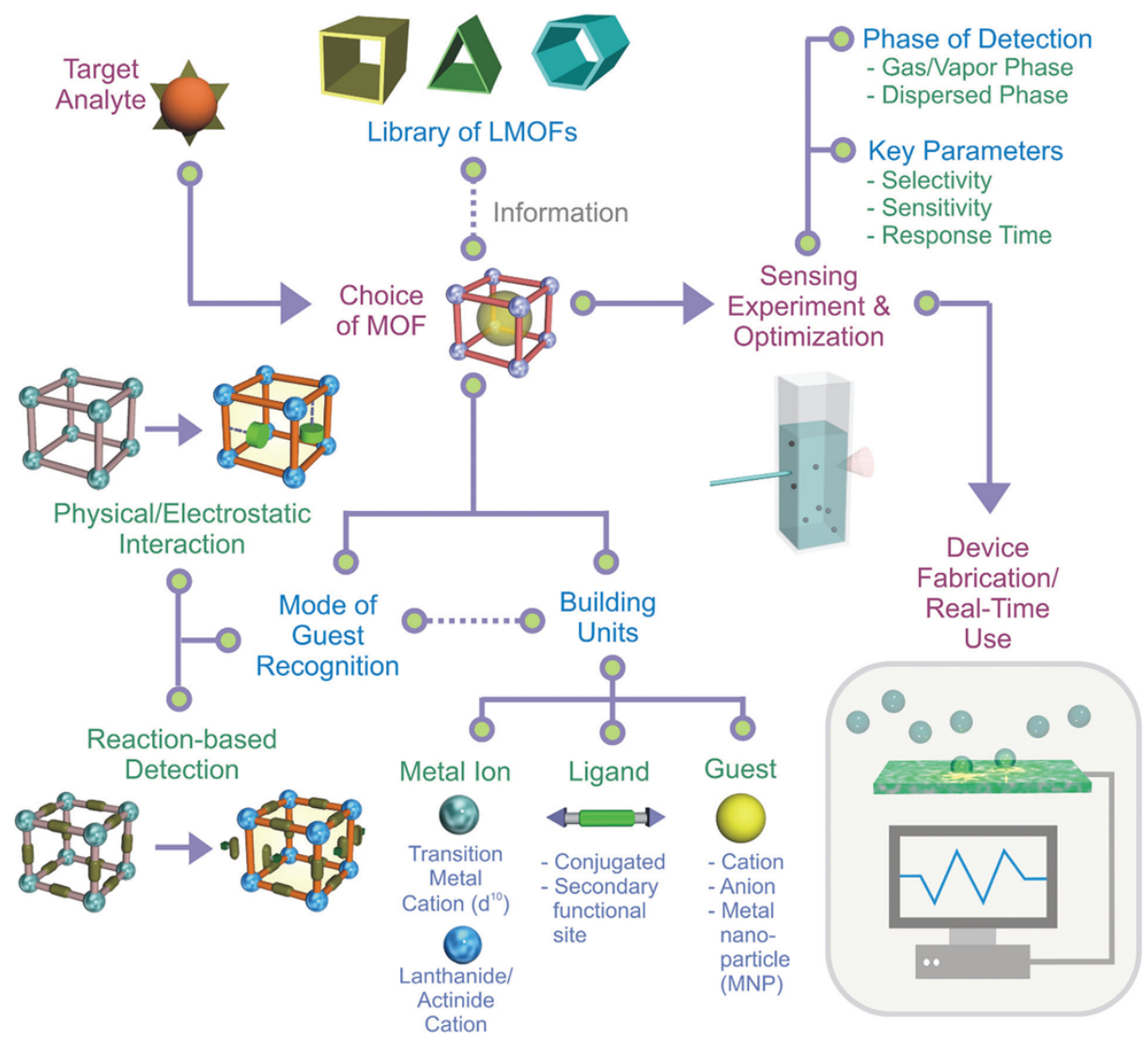

Figure 7.4. Design and development of advanced functional materials for sensing application (Lustig et al. 2017, reprinted with permission from the Royal Society of Chemistry).

\subsubsection{Advancing Volume, Density, and Flow Measurement Technologies}

In nuclear fuel recycling processes such as the PUREX and pyroprocessing technologies, as well as in some MSR applications, nuclear material is present in bulk fluids. Methods to detect and analyze the concentrations of nuclear materials in the fluids are critical as discussed above. However, those analytical approaches can inform operators of the concentrations in the material sample, but there is no way to complete a material balance without some knowledge of the fluid volume. One method to monitor fluid level and density in a tank is to suspend bubbler dip-tubes in the liquid. In this approach, gas is forced down a dip-tube and bubbles into the process fluid as shown in Figure 7.5. The pressures within the tubes are measured and represent contributions from the hydrostatic pressure $\left(P_{\mathrm{h}}\right)$ and the excess bubble pressure $\left(P_{\mathrm{B}}\right)$, which is a function of the dip-tube geometry and the fluid properties. The main advantages to bubbler approaches are high measurement accuracy, limited moving parts, and radiation hardiness. 


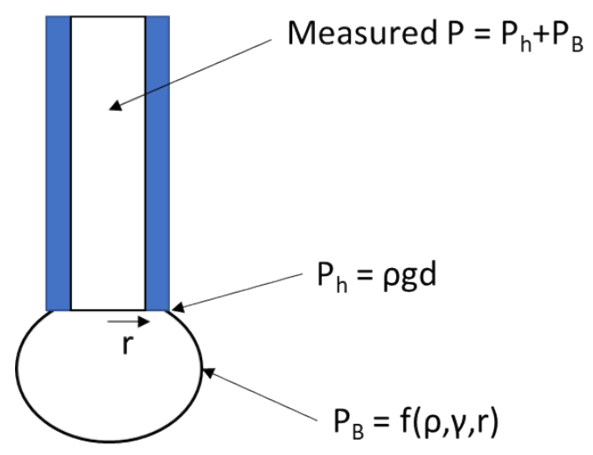

Figure 7.5. Illustration of the bubbler concept. (Courtesy of INL.)

Multiple bubbler approaches including double (ISO 2008; Hosoma 2010), triple (Williams et al. 2021; US 2021/0310923), and more recently dynamic single (Kim et al. 2017, 2020) bubblers have been explored. The most mature of the approaches is a double bubbler, which has been used in aqueous reprocessing facilities to determine the total volume (level and density) of solution in the dissolver/input accountancy tank with uncertainties less than 1\% (ISO 2008; Hosoma 2010). Similar accuracies (albeit with significantly less data) have been demonstrated using triple and dynamic single bubblers.

\section{Core Idea}

New electrochemical sensor technologies will advance pyrochemical processing approaches to recycling of metallic fuels and cleanup and restoration of MSR fuels.
However, there are limitations to a bubbler approach to measure fluid level and density. The main challenges include corrosion of the tips, buildup of material in the tubes, and plugging of the tubes. Frequent maintenance and calibration are required to maintain the high level of accuracy. For example, in aqueous applications daily checks, frequent acid washes/blowouts, and monthly calibration are required. In molten salts applications

plugging (Williams et al. 2021) has been a major hurdle, and recalibration in situ has not been demonstrated. Development of more robust and innovative dip-tube materials/coatings, operating parameters, and in situ calibration or calibration-free approaches are necessary.

Additional limitations with current approaches arise in that the fluid must be a single phase, homogenous, and at static and thermal equilibrium (ISO 2008). As a result, in applications in which mixing or flow is present (molten salt reactors, mixer settler tanks, etc.), the flow near the dip-tubes disrupts the bubble formation and distorts current modeling methods and statistical methods. Innovative engineering solutions and $R \& D$ are necessary to bridge the gap between the current bubbler approaches and alternative systems that can be applied in more complex flow conditions.

To achieve accurate accounting of material in nuclear separation processing system, there is a general need to improve the bubbler technology, and more broadly, there is need to develop new more robust methods for measuring liquid volumes, densities, and flowrates. Accurate data concerning these parameters, coupled with the real-time chemical analytical methods discussed above will enable accurate real-time tracking of SNM to revolutionize plant process control and safeguards regimes.

\subsection{References}

Andrade, J. M.; Cristoforetti, G.; Legnaioli, S.; Lorenzetti, G.; Palleschi, V.; Shaltout, A. A. Classical Univariate and Partial Least Squares for Quantitative Analysis of Brass Samples by LaserInduced Breakdown Spectroscopy. Spectrochimica Acta Part B 2010, 65, 658-63. 
Barefield, J. E.; Judge, E. J.; Berg, J. M.; Willson, S. P.; Le, L. A.; Lopez, L. N. Analysis and Spectral Assignments of Mixed Actinide Oxide Samples Using Laser-Induced Breakdown Spectroscopy (LIBS). Applied Spectroscopy 2013, 67, 433-40.

Boglaienko, D.; Andersen, A.; Heald, S. M.; Varga, T.; Mortensen, D. R.; Tetef, S.; Seidler, G. T.; Govind, N.; Levitskaia, T. G. X-Ray Absorption Spectroscopy of Trivalent Eu, Gd, Tb, and Dy Chlorides and Oxychlorides. Journal of Alloys and Compounds 2022, 897, 162629.

Boglaienko, D.; Seidler, G. T.; Dean-Hill, D. J.; Levitskaia, T. G. In situ Molten Salt Studies in a Laboratory X-ray Absorption Spectrometer Equipped with a Vacuum Furnace. Presented by D. Boglaienko at XAFS 2021 Virtual, Online Conference, Australia, July 12, 2021. PNNL-SA163106.

Bol'shakov, A. A.; Mao, X.; Gonzales, J. J.; Russo, R. E. Laser Ablation Molecular Isotope Spectroscopy (LAMIS): State of the Art. Journal of Analytical Atomic Spectrometry 2016, 31, 119-34.

Clifford, A. J.; Lackey, H. E.; Nelson, G. L.; Bryan, S. A.; Lines, A. M. Raman Spectroscopy Coupled with Chemometric Analysis for Speciation and Quantitative Analysis of Aqueous Phosphoric Acid Systems. Anal Chem 2021, 93(14), 5890-96.

Devkota, J., Kim, K.-J., Ohodnicki, P., Culp, J. T., Greve, D.W., Lekse, J. W. Zeolitic Imidazolate Framework-Coated Acoustic Sensors for Room Temperature Detection of Carbon Dioxide and Methane. Nanoscale 2018, 10, 8075-78.

Ditter, A. S.; Holden, W. M.; Cary, S. K.; Mocko, V.; Latimer, M. J.; Nelson, E. J.; Kozimor, S. A.; Seidler, G. T. Resonant Inelastic X-ray Scattering using a Miniature Dispersive Rowland Refocusing Spectrometer. Journal of Synchrotron Radiation 2020, 27, 446-54.

EasyXAFS. EasyXAFS. https://www.easyxafs.com/ (accessed 2022-01-28).

Emmert, L. A.; Chinni, R. C.; Cremers, D. A.; Jones, C. R.; Rudolph, W. Comparative Study of Femtosecond and Nanosecond Laser-Induced Breakdown Spectroscopy of Depleted Uranium. Applied Optics 2011, 50, 313-17.

Fichet, P.; Mauchien, P.; Moulin, C. Determination of Impurities in Uranium and Plutonium Dioxides by Laser-Induced Breakdown Spectroscopy. Applied Spectroscopy 1999, 53, 1111-17.

Fredrickson, G. L. ; Cao, G.; Tripathy, P. K.; Shaltry, M. R.; Herrmann, S. D.; Yoo, T.-S.; Karlsson, T. Y.; Horvath, D. C.; Gakhar, R.; Williams, A. N.; Hoover, R.O.; Phillips, W. C.; Marsden, K. C. Review-Electrochemical Measurements in Molten Salt Systems: A Guide and Perspective. Journal of The Electrochemical Society 2019, 166, D645-D659.

Heller, F. D.; Casella, A. J.; Lumetta, G. J.; Nash, K. L.; Sinkov, S. I.; Bryan, S. A. Incorporating Spectroscopic On-line Monitoring as a Method of Detection for a Lewis Cell Setup. Analyst 2017, 142(13), 2426-33.

Hosoma, T. Volume Measurement System for Plutonium Nitrate Solution and its Uncertainty to be Used for Nuclear Materials Accountancy Proved by Demonstration over Fifteen Years. JAEA Research 2010-033.

Hoyt, N. C.; Launiere, C. A.; Stricker, E. A. In-Process Monitoring of Molten Salt Composition by Voltammetry and Automated Sampling-Based Techniques, Journal of Nuclear Materials Management 2021, 49, 87-98.

Iizuka, M.; Inoue, T.; Shirai, O.; Iwai, T.; Arai, Y. Application of Normal Pulse Voltammetry to On-Line Monitoring of Actinide Concentrations in Molten Salt Electrolyte, Journal of Nuclear Materials 2001, 297, 43-51. 
ISO 18213 Parts 1-6, Nuclear Fuel Technology Tank Calibration and Volume Determination for Nuclear Materials Accountancy, First edition, March 15, 2008.

Jahrman, E. P.; Holden, W. M.; Ditter, A. S.; Mortensen, D. R.; Seidler, G. T.; Fister, T. T.; Kozimor, S. A.; Piper, L. J.; Rana, J.; Hyatt, N. C.; Stennett, M. C. An Improved Laboratory-Based X-Ray Absorption Fine Structure and X-Ray Emission Spectrometer for Analytical Applications in Materials Chemistry Research. Review of Scientific Instrumentation 2019, 90, 024106.

Kim, J.; Bae, S.; Park, T.; Paek, S.; Kim, T.; Lee, S. Wireless Simultaneous System for Liquid Level and Density Using Dynamic Bubbler Technique: Application to $\mathrm{KNO}_{3}$ Molten Salts. Industrial \& Engineering Chemistry Research 2020, 82, 57-62.

Kim, J.; Lee, J.; Bae, S.; Paek, S.; Kim, H.; T. Kim; T. Park Automated High-Temperature Liquid Level Measurement System Using a Dynamic Tube Pressure Technique. Journal of Industrial and Engineering Chemistry 2017, 49, 30-35.

Lines, A. M.; Adami, S. R.; Sinkov, S. I.; Lumetta, G. J.; Bryan, S. A. Multivariate Analysis for Quantification of Plutonium(IV) in Nitric Acid Based on Absorption Spectra. Analytical Chemistry 2017, 89(17), 9354-59.

Lines, A. M.; Hall, G. B.; Asmussen, S.; Allred, J.; Sinkov, S.; Heller, F.; Gallagher, N.; Lumetta, G. J.; Bryan, S. A. Sensor Fusion: Comprehensive Real-Time, On-Line Monitoring for Process Control via Visible, Near-Infrared, and Raman Spectroscopy. ACS Sensors 2020b, 5(8), 2467-75.

Lines, A. M.; Hall, G. B.; Sinkov, S. I.; Levitskaia, T.; Gallagher, N. B.; Lumetta, G. J.; Bryan, S. A. Overcoming Oxidation State Dependent Spectral Interferences: On-Line Monitoring of U(VI) Reduction to U(IV) via Raman and UV-vis Spectroscopy. Industrial \& Engineering Chemistry Research 2020a, 59, 8894-901.

Lumetta, G. J.; Allred, J. R.; Bryan, S. A.; Hall, G. B.; Levitskaia, T. G.; Lines, A. M.; Sinkov, S. I. Simulant Testing of a Co-Decontamination (CoDCon) Flowsheet for a Product with a Controlled Uranium-to-Plutonium Ratio. Separation Science and Technology 2019, 54(12), 1977-84.

Lustig, W. P., Mukherjee, S., Rudd, N. D., Desai, A. V., Li, J., and Ghosh, S. K. Metal-Organic Frameworks: Functional Luminescent and Photonic Materials for Sensing Applications, Chemical Society Reviews 2017, 46, 3242-85.

Oleneva E.; Savosina, J.; Agafonova-Moroz, M.; Lumpov, A.; Babain, V.; Jahatspainian, I.; Legin, A.; Kirsanov, D. Potentiometric Multisensor System for Tetra- and Hexavalent Actinide Quantification in Complex Rare Earth Metal Mixtures related to Spent Nuclear Fuel Reprocessing. Sensors and Actuators B: Chemical 2019, 288, 155-62.

Sarkar, A.; Alamelu, D.; Aggarwal, S. K. Determination of Thorium and Uranium in Solution by LaserInduced Breakdown Spectrometry. Applied Optics 2008, 47, G58-64.

Schroll, C. A.; Lines, A. M.; Heineman, W. R.; Bryan, S. A. Absorption Spectroscopy for the Quantitative Prediction of Lanthanide Concentrations in the $3 \mathrm{LiCl}-2 \mathrm{CsCl}$ eutectic at $723 \mathrm{~K}$. Analytical Methods 2016, 8(43), 7731-38.

Schumacher, P. D.; Doyle, J. L.; Schenk, J. O.; Clark, S. B. Electroanalytical Chemistry of Lanthanides and Actinides, Reviews in Analytical Chemistry 2013, 32, 159-71.

Singh, J. P.; Yueh, F.; Zhang, H.; Carney, K. P.; Krsul, J. A Preliminary Study of the Determination of Uranium, Plutonium, and Neptunium by Laser-Induced Breakdown Spectroscopy. 1996, US Department of Energy, DIAL 10575. 
Sirven, J.; Pailloux, A.; M'Baye, Y.; Coulon, N.; Alpettaz, T.; Gosse, S. Towards the Determination of the Geographical Origin of Yellow Cake Samples by Laser-Induced Breakdown Spectroscopy and Chemometrics. Journal of Analytical Atomic Spectrometry 2009, 24, 451-59.

Smith, A, L; Verleg, M. N.; Vlieland, J.; de Haas, D.; Ocadiz-Flores, J. A.; Martin, P.; Rothe, J.; Dardenne, K.; Salanne, M.; Gheribi, A. E.; Capelli, E.; van Eijcka, L.; Konings, R. J. M. In situ High-Temperature EXAFS Measurements on Radioactive and Air-Sensitive Molten Salt Materials. Journal of Synchrotron Radiation 2019, 26, 124-36.

Timoshenko, J.; Frenkel, A. I. "Inverting” X-ray Absorption Spectra of Catalysts by Machine Learning in Search for Activity Descriptors. ACS Catalysis 2019, 9, 10192-211.

Tylka, M. M.; Willit, J. L.; Prakash, J.; Williamson, M. A. Method Development for Quantitative Analysis of Actinides in Molten Salts. Journal of the Electrochemical Society 2015, 162, H625H633.

US 2021/0310923. Triple Bubbler System, Fast-Bubbling Approach, and Related Methods. Published October 7, 2021.

Wachter, J. R.; Cremers, D. A. Determination of Uranium in Solution Using Laser-Induced Breakdown Spectroscopy. Applied Spectroscopy 1987, 41, 1042-48.

Williams, A. N.; Cao, G.; Westphal, B.; Galbreth, G. G.; Sanders, J.; King, J.; Sell, D.; Li, S.; Gese, N.; Serrano-Rodriguez, B. Development of Safeguards Instrumentation and Techniques at the Idaho National Laboratory for an Electrochemical Facility. Journal of Nuclear Materials Management 2021, XLIX, No. 1, 99-115.

Williams, A. N. Measurement of Rare Earth and Uranium Elements Using Laser-Induced Breakdown Spectroscopy (LIBS) in an Aerosol System for Nuclear Safeguards Applications. PhD Dissertation, Virginia Commonwealth University, 2016. https://doi.org/10.25772/KD67-5531.

Wu, J.; Qiu, Y.; Li, X.; Yu, H.; Zhang, Z.; Qiu, A. Progress in Laser-Induced Breakdown Spectroscopy in Nuclear Industry Applications. Journal of Physics D: Applied Physics 2020, 53. DOI: 10.1088/1361-6463/ab477a. 


\section{FRD 7: Achieve Control of Redox Potential in Molten Salt Systems}

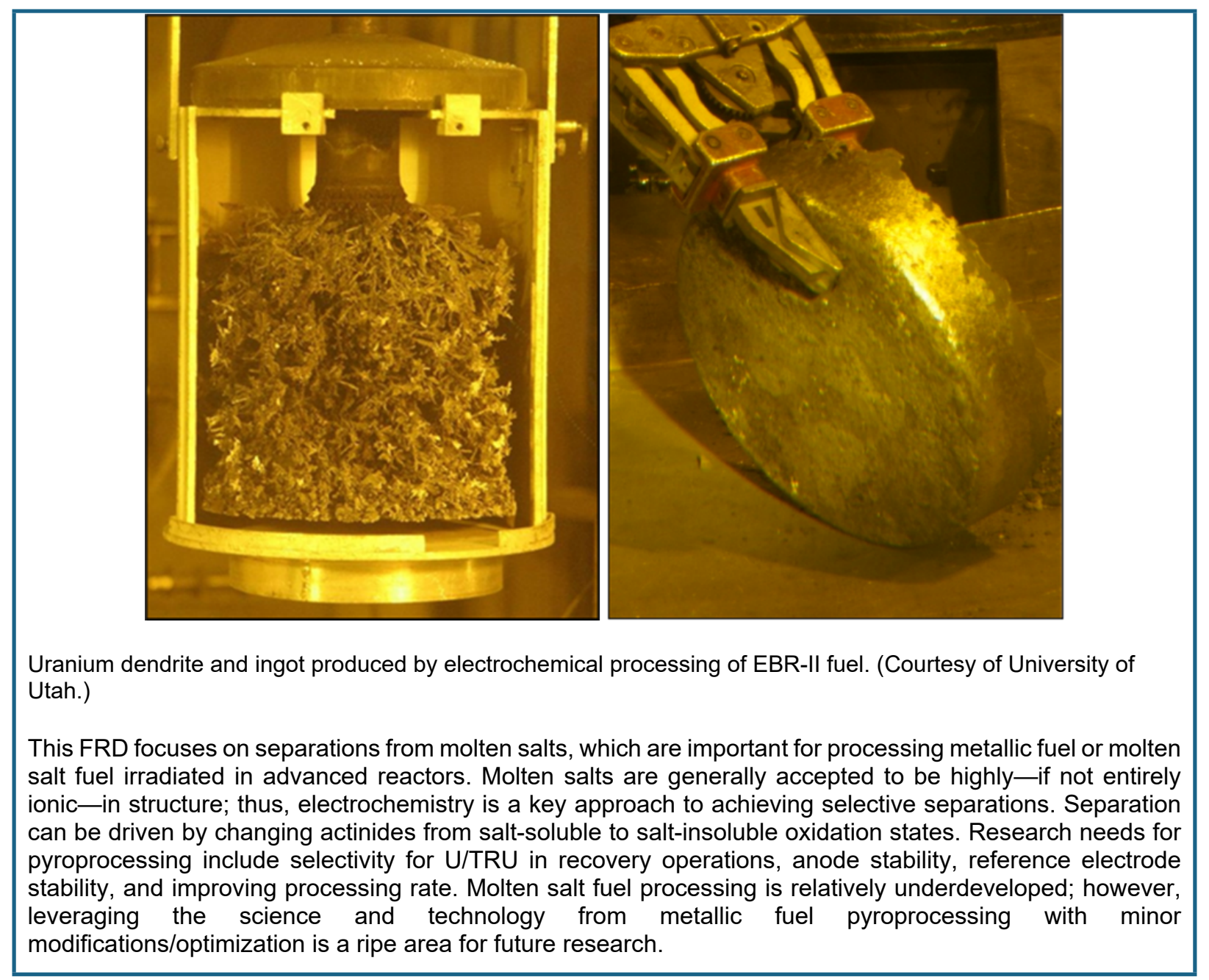

\subsection{Background}

Electrochemistry is fundamentally important for enabling two advanced nuclear energy applications: electrorefining of metallic fuel and operation of MSRs, supporting both metal-fueled and molten salt-fueled advanced nuclear power reactors, which both involve the use of molten salts. The foundational objective is to minimize cost and maximize efficiency of actinide separations for either of these types of fuels. Removing FPs from molten salts or concentrating them into waste forms is also an important objective for both systems. The base molten salt mixtures for these two systems are different. Electrorefiners use eutectic $\mathrm{LiCl}-\mathrm{KCl}$ as the base salt. Molten salt reactors may utilize a wide range of different salts comprised of either chlorides or fluorides and metal ions such as $\mathrm{Na}^{+}, \mathrm{K}^{+}, \mathrm{Mg}^{2+}$, and $\mathrm{Ca}^{2+}$. This is not meant to be an exhaustive list, as each reactor developer chooses the salt mixture based on their own specific requirements.

Reduction-oxidation (redox) chemistry and electrochemistry are tightly intertwined in the highly ionic molten salt mixtures. Separations can be driven by controlling the redox potential, which affects the oxidation state of metals of interest. For various metals, some oxidation states are soluble in molten salts, while others are insoluble. It is even possible to drive metals into volatile forms by achieving high oxidation 
states. Uranium $(\mathrm{U})$, for example, can form volatile $\mathrm{UF}_{6}$ in a fluoride salt mixture and volatile $\mathrm{UCl}_{4}$ in a chloride salt mixture. Active electrochemical control is important to drive changes in the equilibrium state of molten salts. But in this FRD, we also consider the utility of adding oxidizing or reducing agents that dissolve in the salt and react. The benefits of these separations include increasing utilization of actinide fuels, minimizing long-term radiological impact on geological repositories, and minimizing the volume of waste that requires geological disposal.

\subsubsection{Electrorefining Metallic Fuel}

Electrorefining of metallic $\mathrm{U}$ or $\mathrm{U}-\mathrm{Zr}$ or $\mathrm{U}-\mathrm{Pu}-\mathrm{Zr}$ alloys has been researched and applied for many years and is the main unit operation for processing irradiated Experimental Breeder Reactor-II (EBR-II) fuel at the Fuel Conditioning Facility at INL (Li et al. 2005, 2006). That fuel-treatment campaign has been ongoing since 2000 and uses two engineering-scale electrorefiners. The function of electrorefining in this application is to recover purified $U$ metal from the irradiated fuel. Bond sodium metal is reacted in the process to form $\mathrm{NaCl}$, which accumulates in the electrorefiner salt pools. The EBR-II spent fuel treatment project has been focused on safely dispositioning the irradiated fuel from EBR-II, but the research performed with the electrorefiners dating back to the mid-1990s has been considered valuable for future endeavors such as processing of commercial reactor fuel in the United States, South Korea, and Japan.

Applying the electrorefining pyroprocessing technology to the processing commercial power reactor spent fuel is also a subject of interest. Light water reactor spent fuel is in the form of $\mathrm{UO}_{2}$, which must first be converted to metallic form before processing in an electrorefiner. The electrolytic reduction process for $\mathrm{UO}_{2}$ was thus developed starting in the early $2000 \mathrm{~s}$ to prepare commercial spent fuel for electrorefining (Herrmann et al. 2007). Electrolytic reduction was included with electrorefining in experiments done in the US-Republic of Korea Joint Fuel Cycle Study. The recent announcement of plans by TerraPower and GEHitachi to build a demonstration power-generating ( $345 \mathrm{MWe}$ ), sodium-cooled, metallic-fuel reactor called Natrium means that there could be a direct application of electrorefining to future commercial nuclear fuel cycles. Although there are currently no plans to reprocess Natrium fuel, if such a decision were made to move toward a closed the nuclear fuel cycle, metallic-fuel electrorefining would clearly be the processing technology of choice.

\subsubsection{Molten Salt-Fueled Reactors}

Molten salt-fueled nuclear reactors were first developed in the 1960s at ORNL under the MSRE (Rosenthal et al. 1970). That experiment was ended by the early 1970s, and interest in MSRs waned in the 1970s through the early part of this century. In the last decade or so, however, several companies have announced plans to design new MSRs. This includes Flibe Energy, TerraPower, Terrestrial Energy, Elysium Industries, Moltex, and several others. Some companies are working on fast neutron-spectrum reactors with chloride salts as the fuel/coolant, while others are looking at thermal neutron-spectrum reactors with fluoride salts as the fuel/coolant. Common to most of the proposed systems is a primary salt loop that transports salt between a core and heat exchangers. The systems need to be controlled with respect to fluid flow, salt composition, and temperature distribution.

Redox chemistry and electrochemistry are needed in these MSR systems to monitor and control the salt composition and corrosivity of the salt. When fission occurs in the core, it creates a net oxidizing effect. If uncontrolled, the salt will become progressively more corrosive and quickly corrode the metal surfaces that contain the molten salt. Some MSR designs include plans to add salt-processing technology that may serve to separate FPs, actinides, or both from the salt to minimize HLW generation. Electrochemistry and redox control can be powerful tools to drive separation of target metals from MSR fuel salts. Thus, this FRD impacts MSR technology both from the standpoint of operational control and waste management. 


\subsection{Technical Challenges and Research Needs}

\subsubsection{Electrorefining}

Electrorefining systems at a minimum consist of anode, cathode, and electrolyte. See Figure 8.1 for a basic schematic of $U$ electrorefining in molten salt electrolyte. In the case of metallic fuel electrorefining, the electrolyte would be a molten salt mixture. The molten salt base components (typically $\mathrm{LiCl}$ and $\mathrm{KCl}$ in eutectic ratio) are electrochemically more stable than other metal chlorides in the system. Thus, they are not subject to reduction or oxidation. For recovery of $U$ from a metallic alloy-based spent fuel, the following reactions occur.

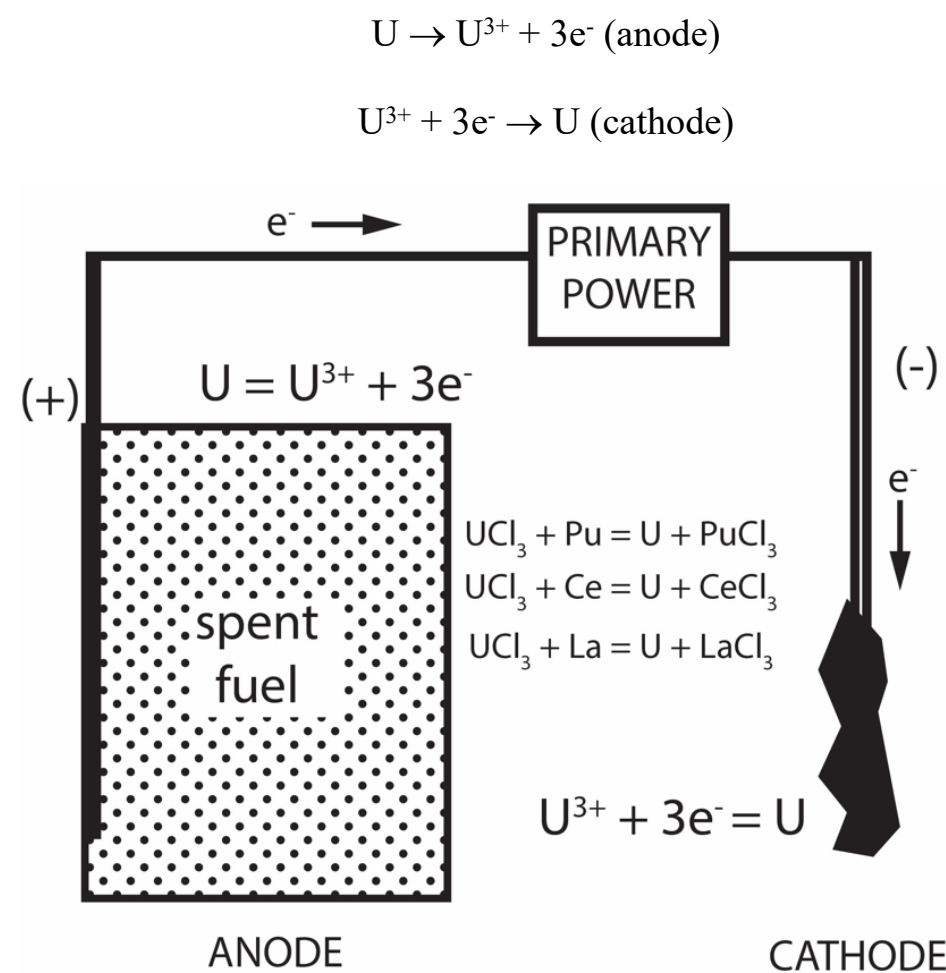

Figure 8.1. Basic schematic for metal fuel electrorefining. Equals signs denote equilibrium signs. In situ application of LIBS and LAMIS can provide a new tool for real-time monitoring of nuclear processing streams for application to plant process and control systems, and safeguards. (Courtesy of University of Utah.)

Anode potential drives oxidation, and cathode potential drives reduction. By keeping the anode and cathode within acceptable ranges, U, TRU elements, and active metals oxidize at the anode, whereas only $U$ reduces at the cathode. A stable thermodynamic reference electrode is needed to monitor the cathode and anode potentials and ensure they stay within the acceptable ranges. An important focus on reference electrode development is to attain long-term potential stability.

Reference electrodes will be discussed in more depth in Section 8.2.4. The active metal FPs include Group I, Group II, and lanthanides. These FPs accumulate in the molten salt phase, as they are known to have solubility in $\mathrm{LiCl}-\mathrm{KCl}$. 
Cathode materials can affect the deposition of metals onto electrorefiner cathodes based on sticking efficiency and metal alloying effects. Typically, steel cathodes are used for $U$ electrorefining that allow for the $U$ to be harvested by pulling out the cathode mandrel. But higher processing rates may be achieved if $\mathrm{U}$ metal forms and falls off the cathode. This can allow for recovery of electrorefined $U$ while the electrorefiner is actively running. Certain liquid metals have been extensively studied for co-deposition of U/TRU actinides. Liquid Cd is a prime example and has been tested at INL in the Fuel Conditioning Facility (Vaden et al. 2008). Solid cathode materials have also demonstrated solution-forming behavior with U. Stika et al. reported that $U$ metal forms a solid solution upon deposition onto Ni cathodes (Stika et al. 2018). Aluminum has also been reported as a reactive cathode for uranium deposition (Cassayre et al. 2007). Murakami and Iizuka proposed an innovative rare earth FP separation process based on electrowinning with a Si cathode, which results in formation of a rare earth silicide (Murakami et al.2020). The effect of forming solutions in either a solid or liquid cathode is that it reduces the activity of deposited metal. This can stabilize the metal on the cathode when it would otherwise tend toward remaining in the salt phase. This is how the liquid $\mathrm{Cd}$ cathode works to recover Pu and $\mathrm{U}$ simultaneously, and an inert cathode will only recover U.

Note that electrorefining is only useful for producing a purified metal from a contaminated metal. It is, thus, only considered for application to metallic fuel processing (or processing of oxide or molten salt fuels after they have been reduced to metal).

For many years, the focus on improving electrorefining has been to increase its rate of processing, and this need persists. Previous efforts have examined ways to continuously recover U product without stopping the electrorefining operation. Argonne National Laboratory developed a high-throughput electrorefiner concept in which $U$ metal could be continuously scraped from cathode plates and recovered from the salt. However, this goal remains elusive. The ability to co-recover U/TRU on an electrorefiner cathode requires use of a cathode metal that will alloy with TRU elements and stabilize them against reoxidation. Argonne developed the liquid $\mathrm{Cd}$ cathode for this purpose, but $\mathrm{Cd}$ is complicated to work with because of its toxicity and high vapor pressure. Consequently, another important focus for electrorefining development is alternative cathode materials for U/TRU recovery with minimum contamination with lanthanides. Emphasis should be placed on solid cathode materials to avoid handling liquid metal cathodes. Examples of solid cathodes that demonstrate interaction with actinides or rare earths are $\mathrm{Al}, \mathrm{Ni}$, and $\mathrm{Si}$.

\subsubsection{Electrolytic Reduction}

As mentioned above, oxide fuels must be reduced to metallic form prior to processing in an electrorefiner. This category of fuel is an important consideration, given that the vast majority of spent fuel currently in existence is in oxide form. The first process developed to achieve this outcome was Li reduction in $\mathrm{LiCl}$ (Simpson et al. 2008). Lithium metal could be added to $\mathrm{LiCl}$ in which $\mathrm{UO}_{2}$ was submerged. The following reaction is spontaneous.

$$
4 \mathrm{Li}+\mathrm{UO}_{2} \rightarrow \mathrm{U}+2 \mathrm{Li}_{2} \mathrm{O}
$$

The problem with this approach is that $\mathrm{Li}_{2} \mathrm{O}$ concentration increases with each batch of fuel reduced. And the higher the $\mathrm{Li}_{2} \mathrm{O}$ concentration is, the lower is the equilibrium yield to metals. Reduction of lanthanide oxides is particularly limited as $\mathrm{Li}_{2} \mathrm{O}$ concentration increases. Thus, the electrolytic reduction process was developed in which there is a constant $\mathrm{Li}_{2} \mathrm{O}$ concentration in $\mathrm{LiCl}$, and the fuel is reduced in a cathode basket while $\mathrm{O}_{2}$ is formed at the anode. The reactions involved with electrolytic reduction are as follows.

$$
\begin{gathered}
\mathrm{UO}_{2}+4 \mathrm{e}^{-} \rightarrow \mathrm{U} \text { (cathode) } \\
4 \mathrm{Li}_{2} \mathrm{O}+4 \mathrm{e}^{-} \rightarrow 4 \mathrm{Li}+2 \mathrm{O}^{2-} \text { (cathode) }
\end{gathered}
$$




$$
\begin{aligned}
4 \mathrm{Li}+\mathrm{UO}_{2} & \rightarrow \mathrm{U}+2 \mathrm{Li}_{2} \mathrm{O} \text { (cathode) } \\
2 \mathrm{O}^{2-} & \rightarrow \mathrm{O}_{2}+4 \mathrm{e}^{-} \text {(anode) }
\end{aligned}
$$

Figure 8.2 shows a schematic for electrolytic reduction. Many papers have been published on this process (Herrmann et al. 2007; Sakamura and Omori 2010; Kim et al. 2017), but there has yet to be a reported operation of such a system with $\mathrm{UO}_{2}$ batch sizes exceeding about $1 \mathrm{~kg}$. Electrolytic reduction does not achieve actual actinide separations but is an essential precursor to electrorefining when considering oxide fuel as the process feedstock.

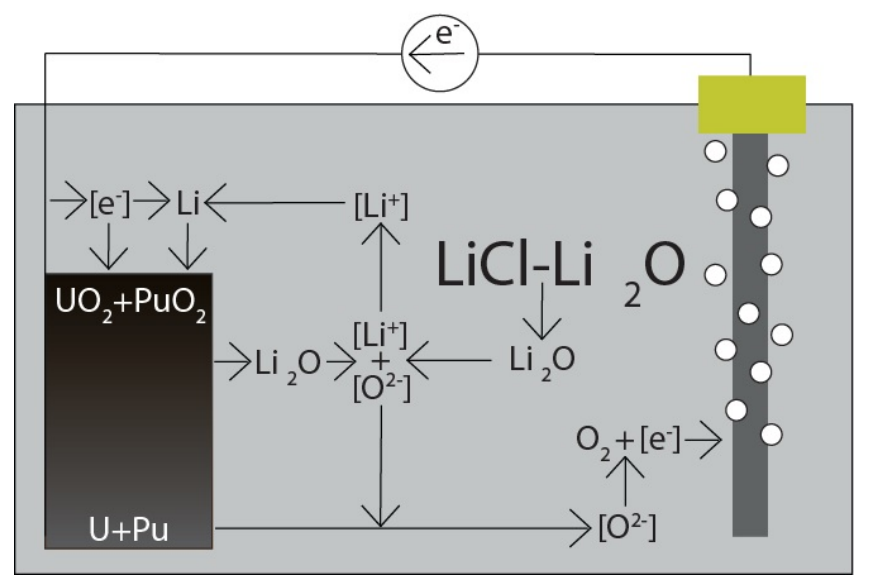

Figure 8.2. Basic schematic for electrolytic reduction of oxide fuel. (Courtesy of University of Utah.)

Similar to electrorefining, electrolytic reduction suffers from low rate of processing. It also requires postprocessing to remove salt from the reduced product, which has yet to be optimized. These are key areas needed to focus research on electrolytic reduction. Linking electrolytic reduction to electrorefining requires near complete removal of $\mathrm{LiCl}$ and $\mathrm{Li}_{2} \mathrm{O}$ from the reduced product. New anode materials are also an important research need for electrolytic reduction. Expensive Pt is currently the only viable option for these anodes. Cheaper, durable anode materials are needed.

\subsubsection{Electrowinning/Electrolysis}

Electrowinning is similar to electrorefining and electrolytic reduction. It results in the formation of a metallic cathode deposit. But what is principally different is that the metal is originally in the salt solution as an oxide or chloride. For example, $\mathrm{U}$ metal can be recovered from dissolved $\mathrm{UCl}_{3}$ via these electrode reactions.

$$
\begin{gathered}
\mathrm{UCl}_{3}+3 \mathrm{e}^{-} \rightarrow \mathrm{U}+3 \mathrm{Cl}^{-} \text {(cathode) } \\
3 \mathrm{Cl}^{-} \rightarrow 1.5 \mathrm{Cl}_{2}+3 \mathrm{e}^{-} \text {(anode) }
\end{gathered}
$$

The anode is designed in this case to be inert. Graphite is an example of an anode material that can be used to generate $\mathrm{Cl}_{2}$. There still may be problems with sweeping the $\mathrm{Cl}_{2}$ out of the system before it can corrode contacting metals. Elemental chorine is highly reactive with Fe-based alloys at the high temperatures used for electrowinning.

Another electrowinning process that has been reported is the Oxide Electrowinning Method (Kofuji et al. 2007). This process has been developed jointly by Japan Atomic Energy Agency and the Russian Research Institute of Atomic Reactor. It starts with reaction of actinide oxides with $\mathrm{Cl}_{2}$ to form actinyl $\left(\mathrm{UO}_{2}{ }^{2+}, \mathrm{PuO}_{2}{ }^{2+}\right.$, 
etc.) cations in molten $\mathrm{NaCl}-2 \mathrm{CsCl}$. Then those cations are reduced onto a cathode to form $\mathrm{UO}_{2}, \mathrm{PuO}_{2}$, etc. This method is primarily intended for processing of MOX fuel.

Electrowinning could be applied to processing of either metallic fuel or MSR fuel. It only requires that the actinides be initially mixed in with a base molten salt such as $\mathrm{LiCl}-\mathrm{KCl}$ or $\mathrm{NaCl}$.

Key research needs for electrowinning are improved anode and cathode materials. Anodes need to be capable of sustained $\mathrm{Cl}_{2}$ generation. A reactive anode can be a good alternative if it consists of cheap materials that can be disposed of or replaced. An example of a reactive anode was demonstrated in work by Bagri and Simpson on galvanic reduction of $\mathrm{UCl}_{3}$ from $\mathrm{LiCl}-\mathrm{KCl}$ (Bagri et al. 2018). They used a $\mathrm{Gd}$ metal anode that generated $\mathrm{GdCl}_{3}$, which reports to the salt and would be simply disposed with the waste salt. As with electrorefining, metals that form alloys or intermetallics with U/TRU other than Cd are needed for co-recovery of $U$ and TRU, and stable thermodynamic reference electrodes are needed.

\subsubsection{Control of Redox Potential in MSR Fuel Salt}

Electrode potential monitoring enables control over reactions occurring at anodes and cathodes in electrochemistry-based separation systems.

\section{Core Idea}

Redox potential in the MSR fuel salt dictates the partitioning of metals between salt soluble salt compounds and salt insoluble metals. It can drive extraction of U/TRU from the fuel salt or drive oxidation of U/TRU metal followed by dissolution into the fuel salt. Properly controlling the potential starts with measuring the potential, which requires stable thermodynamic reference electrodes. The next key ingredient is a means to change the redox potential. In the MSR fuel salt, the $U$ is intended to be in the form of a salt, but its oxidation state can vary and thus must be monitored and controlled.

Reference electrodes are used as a point of reference to the potential of another electrode that is subject to variable potential. They should be based on a thermodynamic potential generated by creating a redox couple at equilibrium. For example, $\mathrm{Ag} / \mathrm{AgCl}$ is often used in molten chloride salts. Inside of chamber contained in a membrane, the $\mathrm{Ag}$ is in equilibrium with $\mathrm{AgCl}$. That sets the potential of the $\mathrm{Ag}$ wire which is connected to the electrochemical control system (potentiostat, power supply, etc.).

The challenge with reference electrodes is to keep the composition of the salt inside of the chamber constant. It must be contained in a closed tube that does not allow for salt to flow between the chamber and the electrolyte. But it must support ionic conduction or else the electrical circuit will be open. For laboratoryscale experimentation, commercially available tubes made of materials such as quartz, Pyrex, and mullite have been used for containing the $\mathrm{Ag} / \mathrm{AgCl}$. But these tubes often break, and potential drift can occur on the scale of hours. Reference electrode materials (membrane, seals, thermodynamic couple) and designs are needed for MSR-relevant compositions (fluoride and chloride salts). And mechanical durability must be high in flowing molten salt systems. If reference electrodes are needed in the core of an MSR, stability under irradiation is another important need.

A reducing agent or cathodic reaction can be used to lower the redox potential of salt and prevent corrosion or even drive separation of U/TRU. Generally, options for reducing agents include solids, salt-soluble species, and gases (Zhang et al. 2018). Options for redox control with each of these classes of reducing agents are described below.

Newton et al. (2020) reported the effectiveness of $U$ and $\mathrm{Zr}$ metal rods for buffering the redox potential of a surrogate MSR salt mixture, $\mathrm{NaCl}-\mathrm{CaCl}_{2}-\mathrm{UCl}_{3}$. Nickel(II) chloride was added to the salt to increase the 
redox potential, and both $\mathrm{U}$ and $\mathrm{Zr}$ rods were effective at reducing the potential below the value before the addition of $\mathrm{NiCl}_{2}$.

Dissolved metals have been used for redox control in molten salts dating back to at least the 1960s when Be metal was added to the $\mathrm{LiF}-\mathrm{BeF}_{2}(\mathrm{FLiBe})$-based fuel salt in the MSRE (Olander 2002). Table 8.1 gives some examples and references for metals dissolved in chloride or fluoride salts. When these metals are oxidized, they form chlorides or fluorides and remain in the salt. The atomic level structure of a molten salt with a dissolved metal is still not understood in a general sense. But Merwin et al. made an interesting discovery that $\mathrm{Li}$ exists at $\mathrm{Li}_{8}$ clusters in molten $\mathrm{LiCl}$ (2016).

Table 8.1. Examples of Metals Dissolved in Molten Salts.

\begin{tabular}{llll}
\hline \multicolumn{1}{c}{ Metal } & \multicolumn{1}{c}{ Salt } & \multicolumn{1}{c}{ Solubility } & \multicolumn{1}{c}{ References } \\
\hline $\mathrm{Li}$ & $\mathrm{LiCl}$ & $0.065 \mathrm{wt} \%(923 \mathrm{~K})$ & Burak and Simpson 2016 \\
$\mathrm{Be}$ & $\mathrm{LiF}-\mathrm{BeF}_{2}(\mathrm{FLiBe})$ & $0.76 \mathrm{~mol} \%(803 \mathrm{~K})$ & Hara et al. 2006 \\
$\mathrm{Mg}$ & $\mathrm{MgCl}_{2}-\mathrm{NaCl}-\mathrm{KCl}$ & $0.03 \mathrm{wt} \%(773 \mathrm{~K})$ & Choi et al. 2019 \\
$\mathrm{Ca}$ & $\mathrm{CaCl}_{2}$ & $2.7 \mathrm{~mol} \%(1073 \mathrm{~K})$ & Shaw and Watson 2009 \\
$\mathrm{Na}$ & $\mathrm{NaCl}$ & $>0.08 \mathrm{~mol} \%$ & Bredig et al. 1955 \\
\hline
\end{tabular}

Demonstration of the lowering of redox potential from addition of soluble metals was published by Choi et al. (2019) for $\mathrm{Mg}$ dissolution in $\mathrm{MgCl}_{2}-\mathrm{NaCl}-\mathrm{KCl}$. Reduction of corrosion by Be dissolution in FLiBe was reported by Keiser et al. (1979). Reduction of corrosion of stainless steel in FLiNaK by Li addition was reported by Choi et al. (2021).

In a fluoride salt, a mixture of $\mathrm{H}_{2} / \mathrm{HF}$ can be used to control the redox potential in salt (Toth et al. 1996; Kelleher et al. 2015). Consider the following reaction, which is essentially corrosion of Cr metal:

$$
\mathrm{Cr}+2 \mathrm{HF} \rightarrow \mathrm{CrF}_{2}+\mathrm{H}_{2(\mathrm{~g})}
$$

Increasing the ratio of $\mathrm{H}_{2}$ to $\mathrm{HF}$ in the gas phase will shift the reaction to the left and thus protect metal surfaces from $\mathrm{Cr}$ oxidation based on Le Chatelier's principle.

For molten chloride fast reactor (MCFR) salts that initially consist of only $\mathrm{NaCl}$ and $\mathrm{UCl}_{3}$, a reducing agent is needed. Perhaps Na metal can be dissolved in low concentrations, but that could end up reducing the $\mathrm{UCl}_{3}$ to $\mathrm{U}$ metal. Uranium metal itself is an intriguing option for the redox buffer and has been shown to work in a simulated MCFR salt (Newton et al. 2020). Since U metal is not known to dissolve in the salt as a metal, mass transfer between the salt and $U$ metal would need to be studied and optimized. Alternatively, externally powered electrochemical cells can be used to reduce redox potential in MCFRs.

\subsection{Summary}

Active redox potential/electrochemistry control is essential for both pyroprocessing of metal fuels and performing separations on MCFR fuels. While metal fuel pyroprocessing has been well developed since the mid-1990s at Argonne and INL via the EBR-II spent fuel treatment program, challenges remain including selectivity for U/TRU in recovery operations, anode stability, reference electrode stability, and processing rate. Molten salt fuel processing is relatively underdeveloped with only aspects of the MSRE to learn from. However, the rise of the MCFR enables us to leverage science and technology from pyroprocessing with minor modifications/optimization to fit molten salt-based fuels. In the MCFR, the focus really is on redox potential and control in order to control whether metals are in the salt phase or form 
solid metals that can be separated. Since the preferred salt composition in the MCFR may be as simple as $\mathrm{NaCl}-\mathrm{UCl}_{3}$, redox potential control options are limited and need to be further researched.

\subsection{References}

Bagri, P.; Ong, J.; Zhang, C.; Simpson, M. F. Optimization of $\mathrm{UCl}_{3}$ and $\mathrm{MgCl}_{2}$ Separation from Molten LiCl-KCl Eutectic Salt via Galvanic Drawdown with Sacrificial Gd Anode. Journal of Nuclear Materials 2018, 505, 149-58.

Bredig, M. A.; Johnson, J. W. Smith Jr., W. T. Miscibility of Liquid Metals with Salts. I. The SodiumSodium Halide Systems 1. Journal of the American Chemical Society 1995, 77(2), 307-12.

Burak, A. J.; Simpson, M. F. Measurement of Solubility of Metallic Lithium Dissolved in Molten LiCl$\mathrm{Li}_{2} \mathrm{O}$. JOM 2016, 68(10), 2639-45.

Cassayre, L.; Malmbeck, R.; Masset, P.; Rebizant, J.; Serp, J.; Soucek, P.; Glatz, J.-P. Investigation of Electrorefining of Metallic Alloy Fuel onto Solid Al Cathodes. Journal of Nuclear Materials 2007, 360(1), 49-57.

Choi, S.; Strianese, A. R.; Dale, O.; Simpson, M. F. Electrochemical Measurements for Assessing Corrosion of Metal Alloys in Molten LiF-NaF-KF and $\mathrm{MgCl}_{2}-\mathrm{NaCl}-\mathrm{KCl}$. JOM 2021, 1-11.

Choi, S.; Orabona, N. E.; Dale, Olivia R.; Okabe, P.; Inman, C.; Simpson, M. F. Effect of Mg Dissolution on Cyclic Voltammetry and Open Circuit Potentiometry of Molten $\mathrm{MgCl}_{2}-\mathrm{KCl}-\mathrm{NaCl}$ Candidate Heat Transfer Fluid for Concentrating Solar Power. Solar Energy Materials and Solar Cells 2019, 202, 110087.

Hara, M.; Hatano, Y.; Simpson, M. F.; Smolik, G. R.; Sharp, J. P.; Oya, Y.; Okuno, K. Interactions between Molten Flibe and Metallic Be. Fusion Engineering and Design 2006, 81(1-7), 561-66.

Herrmann, S.; Li, S.; Simpson, M. F. Electrolytic Reduction of Spent Light Water Reactor Fuel BenchScale Experiment Results. Journal of Nuclear Science and Technology 2007, 44(3), 361-67.

Keiser, J. R.; DeVan, J. H.; Lawrence, E. J. Compatibility of Molten Salts with Type 316 Stainless Steel and Lithium. Journal of Nuclear Materials 1979, 85, 295-98.

Kelleher, B. C.; Dolan, K. P.; Brooks, P.; Anderson, M. H.; Sridharan, K. Batch-Scale Hydrofluorination of $\mathrm{Li}_{27} \mathrm{BeF}_{4}$ to Support Molten Salt Reactor Development. Journal of Nuclear Engineering and Radiation Science 2015, 1(4).

Kim, S.-W.; Heo, D. H.; Lee, S. K.; Jeon, M. K.; Park, W.; Hur, J.-M.; Hong, S.-S.; Oh, S.-C.; Choi, E.Y. A Preliminary Study of Pilot-Scale Electrolytic Reduction of $\mathrm{UO}_{2}$ Using a Graphite Anode. Nuclear Engineering and Technology 2017, 49(7), 1451-56.

Kofuji, H.; Sato, F.; Myochin, M.; Nakanishi, S.; Kormilitsyn, M.; Ishunin, V. S.; Bychkov, A. V. MOX Co-Deposition Tests at RIAR for SF Reprocessing Optimization. Journal of Nuclear Science and Technology 2007, 44(3), 349-53.

Li, S. X.; Johnson, T. A.; Westphal, B. R.; Goff, K. M.; Benedict, R. W. Electrorefining Experience for Pyrochemical Reprocessing of Spent EBR-II Driver Fuel. INL/CON-05-00305. Idaho National Laboratory, 2005.

Li, S. X.; Johnson, T. A.; Benedict, R. W.; Vaden, D.; Westphal, B. R. Integrated Electrorefining Efficiency Test for Pyrochemical Fuel Cycle. INL/CON-06-11510. Idaho National Laboratory, 2006.

Merwin, A.; Phillips, W. C.; Williamson, M. A.; Willit, J. L.; Motsegood, P. N.; Chidambaram, Dev. Presence of Li Clusters in Molten LiCl-Li. Scientific Reports 2016, 6(1), 1-6. 
Murakami, T.; Sakamura, Y.; Uozumi, K.; Iizuka, M. Rare Earth Silicide Formation on Si Electrode in LiCl-KCl Melt to Establish a Novel Used Salt Treatment Process. ECS Transactions 2020, 98, $33-46$.

Newton, M. L.; Hamilton, D. E.; Simpson, M. F. Methods of Redox Control and Measurement in Molten $\mathrm{NaCl}-\mathrm{CaCl}_{2}-\mathrm{UCl}_{3}$. ECS Transactions 2020, 98(10), 19.

Olander, D. Redox Condition in Molten Fluoride Salts: Definition and Control. Journal of Nuclear Materials 2002, 300(2-3), 270-72.

Rosenthal, M. W.; Kasten, P. R.; Briggs, R. B. Molten-Salt Reactors-History, Status, and Potential. Nuclear Applications and Technology 1970, 8(2), 107-17.

Sakamura, Y.; Omori, T. Electrolytic Reduction and Electrorefining of Uranium to Develop Pyrochemical Reprocessing of Oxide Fuels. Nuclear Technology 2010, 171(3), 266-75.

Shaw, S.; Watson, R. Solubility of Calcium in $\mathrm{CaCl}_{2}-\mathrm{CaO}$. ECS Transactions 2009, 16(49), 301.

Simpson, M. F.; Herrmann, S. D. Modeling the Pyrochemical Reduction of Spent $\mathrm{UO}_{2}$ Fuel in a PilotScale Reactor. Nuclear Technology 2008, 162(2), 179-83.

Stika, M.; Padilla, S.; Jarrell, J.; Blue, T.; Cao, L. R.; Simpson, M. Thin-Layer Electrodeposition of Uranium Metal from Molten LiCl-KCl. Journal of the Electrochemical Society 2018, 165(3), D135.

Toth, L. M.; Gat, U.; Del Cul, G. D.; Dai, S.; Williams, D. F. Review of ORNLs MSR Technology and Status. CONF-960639-6. Oak Ridge National Laboratory, 1996.

Vaden, D.; Li, S. X.; Westphal, B. R.; Davies, K. B.; Johnson, T. A.; Pace, D. M. Engineering-Scale Liquid Cadmium Cathode Experiments. Nuclear Technology 2008, 162(2), 124-128.

Zhang, J.; Forsberg, C. W.; Simpson, M. F.; Shaoqiang, G.; Lam, S. T.; Scarlat, R. O.; Carotti, F. Redox Potential Control in Molten Salt Systems for Corrosion Mitigation. Corrosion Science 2018, 144, $44-53$. 


\section{Crosscutting Challenges}

During the panel and plenary discussions of the workshop, it became evident that several key aspects of research needs appear in multiple FRDs and therefore are considered broadly crosscutting. The crosscutting challenges identified by the participants fall into the general areas of modeling and simulation, data science, and radiation effects. As discussed in detail in this chapter, projects addressing specific questions inherent to the FRDs will likely face one or more of the crosscutting challenges.

\subsection{Accelerate the Rate of Innovation by Leveraging Simulation and Modeling}

\subsubsection{Introduction}

Modeling and simulation offer the potential to "turbocharge" the rate of research aimed at new separation technologies relevant to nuclear fuel cycles. Computational techniques have permeated and already accelerate modern-day scientific research at the project level, yet a more holistic vision encompasses the incorporation of modeling and simulation throughout a full cycle of R\&D leading to functional separation systems. This R\&D workflow consists of an explore-designpredict-analyze-optimize paradigm in which modeling and simulation play a central role exploring the free energy of separation processes, designing new materials and molecules tailored for meeting the goals laid out in the FRDs, predicting physicochemical behavior in a separation system, analyzing the multiscale functional attributes of a separation system, and finally optimizing the functional performance to realize an efficient separation.

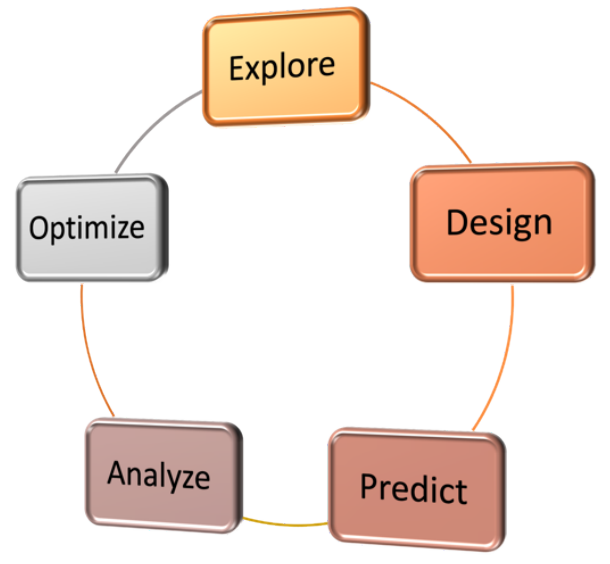

Holistic approach to designing separation systems. (Courtesy Washington State University.)

A holistic approach represents a new vision for modeling and simulation to transform innovation in separations design and optimization for nuclear fuel cycles. While modeling and simulation already have been supporting $R \& D$ at the project level, there is significant potential to accelerate the rate of innovation by incorporating simulation into any and even all aspects of the R\&D workflow. We envision an exploredesign-predict-analyze-optimize approach to modeling and simulation. Exploration entails understanding the underlying free energy landscape of different configurations of atom and molecular positions, as well as chemical compositions so that synthetic pathways for new materials can be developed or material phases and properties can be predicted under extreme conditions (e.g., radiation). Design entails using the knowledge from exploration to identify the key characteristics of a material or separations system of interest, potentially combining information of different phase spaces in new ways (i.e., to design separation systems that are both radiation and fouling resistant). Acting upon design concepts requires prediction of the physicochemical characteristics and performance of a separation, potentially across vastly different length and timescales. Such simulations (which may leverage quantum mechanics, statistical mechanics, or coarse-grained methods) should be performed under realistic conditions that keep the essential interactions needed for predictive viability. The results of such simulations will produce large amounts of data, and 
given the nonequilibrium and nonideal character of the physical systems, new analysis methods may need to be developed that analyze the relationships between structure, property, and reactivity that may potentially lead to new theories or models of behavior. Finally, once new relationships have been identified, it will be possible to optimize the specific properties of interest for a system.

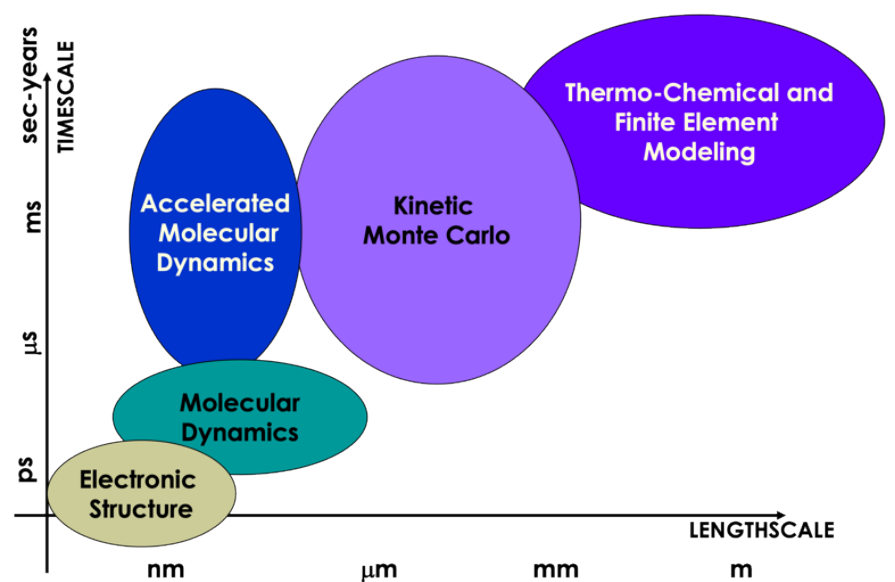

Figure 9.1. A selection of simulation approaches across length scales. (Courtesy of Washington State University.)
Underpinning this approach is the notion that there is immense opportunity for learning how to pass information across different scales of simulation capability (Figure 9.1). The complexity of separation systems in the nuclear fuel cycle uniquely leads to situations in which all levels of information about a system (molecular to macro) can be relevant. For example, the oxidation and reduction potentials governed by the electronic structure of a material influence the reactions that underpin corrosion (the electronic structure scale). Corrosion can be described not only at an atomistic level (using electronic structure and molecular dynamics) but also at a larger scale in the context of the cracks

and defects that corrosion creates that ultimately influence the physical stability and durability of material over long timescales (kinetic Monte Carlo and finite-element methods). Data science methods may be able to aid with this transfer of information, as has been discussed by Clark et al. (2021). Lessons can be learned from recent successes within the materials and crystal-design community that have leveraged different scales of information from the methods shown in Figure 9.1 to explore chemical space and identify structures of materials with specific properties and reactivity (Xie and Grossman 2018).

\subsubsection{Exploring Chemical Space}

Designing and synthesizing robust separation agents (FRD 3 and FRD 5) and innovative approaches that modulate physical phenomena and chemical conditions (FRDs 2, 4, and 7) would benefit from methodologies that explore the chemical space of the system. This means an understanding of the free energy landscape that governs stability of a material (e.g., phase stability and speciation in solution) and how this landscape changes as a function of the conditions that the system experiences. The energy landscape, first introduced in the transition-state theory of molecular reactions, underlies the theoretical formulation of virtually all chemical processes (including materials transformations). Exploring chemical space can encompass the following activities and may be executed in high-throughput and even ML paradigms when integrated with modeling and simulation:

- The prediction of the relative energies of molecules and materials under different compositional constraints (i.e., the prediction of multicomponent alloys, or in molecular design for separations agents). This amounts to finding the structures of molecules and materials at minima on the energy landscape. Importantly, the relative energetics of these minima may change, and minima may even disappear under different conditions (as may occur for the preferred conformations of molecules under different solution conditions, which may influence speciation, aggregation, and the resulting macroscopic solution properties [e.g., viscosity]).

- Calculating the energetic barriers for chemical reactions between minima, which allows for the prediction of kinetics. These barriers can also be incredibly sensitive to external conditions (like the 
solution conditions, chemical potential, temperature, and pressure). Further, identifying the barrier for chemical and materials transformation amounts to elucidating the reaction mechanism for the transformation, which is key to understanding the time evolution or aging of a system under the potentially extreme conditions associated with separations in the nuclear fuel cycle.

A variety of computational methods exist for exploring different aspects of the free energy landscape that allow for the exploration of chemical space. Seamlessly crossing length and timescales relies on coupling atomistic simulation techniques, such as molecular dynamics with advanced sampling methods capable of capturing rare events such as metadynamics or forward-flux sampling. Model reduction is also a useful tool that may increase simulation time by coarse-graining the dynamics of the system. Using such approaches, it would be possible to predict a variety of phenomena related to the FRDs, including the accumulated damage to surfaces (FRD 1), speciation and reactivity in extreme conditions (FRD 2), and the response of a system to different physical stimuli (FRDs 3-5).

\subsubsection{Designing Materials and Molecules}

Using information about the thermodynamic preferences of different materials and molecules, alongside knowledge of their kinetics, provides the foundation for the design of new materials and molecules that underpin separations strategies. Knowledge of the free energy landscape and chemical space exploration have inspired many exciting developments in materials synthesis (DOE-SC 2016) that have paved the way for new separations processes (NAS 2019) in recent decades. In separations domains outside of nuclear chemistry, new types of polymers, mixed matrix membranes, and adsorbents have emerged - all of which have expanded the separations tool kit across a broad range of application space. Yet the design of materials and molecules for separations within the nuclear fuel cycle can often impart unique constraints or desired properties that can complicate simplified representations of chemical reactivity as they may involve excitedstate phenomena, extreme variations of behavior across timescales (imagine the ultrafast ionization caused by $\alpha$ radiation and the long-timescale aging induced by slow creep processes), and chemical extremes of $\mathrm{pH}$, temperature, or pressure. Designing materials that can respond favorably to large doses of ionizing radiation would significantly benefit processing of high-level radioactive wastes and requires accurate simulation of ultrafast radiation dynamics and nonequilibrium behavior. Computational approaches like real-time time dependent DFT (Goings et al. 2018) within an Ehrenfest dynamics formalism have been shown to be effective at modeling ultrafast dynamics after ionization processes in aqueous solutions (Lu et al. 2020), and extensions to more complex chemical environments are needed to understand radiolytic degradation within separations systems (FRD 3 and FRD 5).

Sampling the energy landscape to identify metastable materials and molecules is also important because these metastable species may be operative under realistic separation conditions (FRD 3). Modeling and simulation can provide insight into how external stimuli or system conditions can control the persistence of metastable materials and influence reactivity and performance. Existing approaches, such as cluster expansion methods, create an effective Hamiltonian to reproduce quantum mechanical results to efficiently sample configurational, vibrational, and electronic excitations during the sampling associated with phase diagrams of single and multicomponent materials. There is significant need to develop phase-diagram information for solid, liquid, and gas solutions as indicated by the breadth of complexity encountered within the FRDs. Design approaches must also consider materials performance lifetimes and recyclability at extreme conditions, mandating simulation tools that extend over very long timescales. Highly selective separations approaches can be designed using materials at the molecular- and supramolecular-length scales via chelation, which requires accurate determination of thermodynamic properties (relative energies between minima on an energy landscape). Although electronic-structure methods currently can achieve chemical accuracy $(1 \mathrm{kcal} / \mathrm{mol})$, ML methods are accelerating predictive power so that accurate electronic and thermodynamic properties can be determined for large suites of chemical compounds and materials (Ramakrishnan et al. 2015). From these examples, the computational design of materials and molecules 
with designer separation properties should be expanded beyond the traditional concepts of ground-state selectivity, so that we can ultimately tailor materials and molecules for targeted separations and performance under highly nonideal and chemically extreme environments.

\subsubsection{Predicting Physicochemical Behavior}

The accurate prediction of chemical and materials properties is essential to the entire workflow of how modeling and simulation may be implemented within the designated FRDs. The physicochemical characteristics of a material can derive from fundamental physics at the level of correlation of electrons to the accumulated ensemble of structures that emerge from a material as the result of reactions over time that lead to aging and fouling. At the level of isolated molecules, if we know which molecules are relevant within the complex ensemble of species that occur within a separation system (from the energy landscape), then many fundamental electronic-structure tools exist to accurately predict electrochemical properties and the response and excited-state characteristics that underpin spectroscopic measurement. High electron correlation and relativistic effects can be essential to accurately predict the properties of heavy elements, but many recent advances and open-source software now support the robust use of the right methodology for the right reason. The challenge is that often these signatures of reactivity, or chemical signatures used for monitoring, are highly sensitive to the complex environment that a molecule resides within. For example, if distribution of environments about a metal (different coordination numbers, preferential solvation, etc.) exist within a separation system, then the overall spectroscopic or electrochemical signatures will be an ensemble average of all of the properties of those individual environments (weighted by their relative percent observation). We envision using modeling and simulation to develop libraries of spectroscopic signatures for the ensemble of potential species under separations conditions, which will subsequently enable uncertainty quantification (FRDs 5-7).

Methods of computing properties and processes that cannot yet be accurately and efficiently computed with atomistic and first-principles methods are also needed. In the gas-phase methods like GCMC are effective at determining adsorption isotherms (FRD 3), though there is significant need for method development to access multiple components not only in the gas phase but also under condensed-phase adsorption conditions. This necessarily involves new methods to incorporate multicomponent many-body correlations (meaning nonideality) and the effects of different fields that may occur naturally within the system because of high ionic strength or that may be applied as external stimuli (FRDs 3-5). It remains a singular challenge to develop robust validation strategies to establish the "right" level of theory to describe the multitude of different physicochemical properties of interest to a separations system (either in its design, operation, or optimization) in an integrated manner.

\subsubsection{Data Analysis to Reveal the Fundamental Science of Multidimensional Correlations}

The correlations between particle interactions and hierarchical characteristics (e.g., fractal dimension, effective diameter, and intermolecular networks) can be simulated using a variety of methods, like the lubrication or flow-discrete element method. Within such simulations, mimicking the behavior of a real systems, many variables of the separation system are correlated with not only with one another, but with groups of other variables. ML tools, statistical analyses, and new applied mathematics methods play an integral role in identifying these relationships within the modeling and simulation workflow. Many new methods have been developed for the analysis of simulations of complex solutions, interfaces, and solids that enable the identification of correlated phenomena and a more detailed ability to test the separation of length and timescales that often underpin multiscale computational approaches to explore and design new separations systems. These include graph-theoretical techniques, clustering, genetic algorithms, and other data-mining methods that can extract more information from simulations than traditionally employed correlation functions. Yet often ML methods are focused solely upon identifying the "how" of these relationships and not the "why." While significant progress toward innovative chemical systems can benefit 
without understanding why a given structure-property relationship exists, ultimately this can lead to unknowns that impart significant risk.

For example, rare events can produce unexpected energy transitions that can drive surprising chemical effects. Ionizing radiation produces an avalanche of secondary particles (Crosscut 3 and FRD 3). The most important particles are low-energy electrons, solvated electrons, neutral radicals, radical ions, $\alpha$ and $\beta$ particles, neutrons, and $\gamma$ radiation. These particles can initiate a cascade of secondary electrons. These secondary electrons can drive unexpected chemical speciation and reactivity including corrosion. Although an ML algorithm applied to a suite of simulation data may identify that ionizing radiation is positively correlated with corrosion, the underpinning knowledge about the reaction mechanisms that lead to corrosion are not elucidated. As such, ML cannot provide information about potential strategies to influence radiation reactivity to avoid corrosion processes through manipulation of the intervening reaction pathways. Consequently, it is imperative that physics and chemistry-motivated analysis schemes are employed to yield new theories that drive the behavior of separations systems. Geometric analysis, computational topology, topological data analysis, and graph theory are all methods that support interpretability in the context of known physics and chemical principles (Clark et al. 2021).

\subsubsection{Optimizing Performance}

Optimization may occur for a single desired materials property or across an entire separation system. Importantly, the complexity of a separation system often imparts complex correlations of variables and properties that need to be identified during the analysis phase of the workflow. There is significant opportunity to learn new fundamental physics and chemistry in this process that should not be overlooked because the basic science can inform more advanced simulation strategies that accelerate the discovery, design, and optimization process.

Optimizing the performance of large portions of a separations systems crosscuts all FRDs and coalesces the information from the other stages of the simulation workflow, bridging atomistic mechanisms to the collective responses of hierarchical architectures under coupled extremes. The goal is to use, for example, information about the intermolecular interactions of solvents, the free energy landscape of chemical reactivity, and aggregation thermodynamics and kinetics up to the mesoscale to limit fouling in new separations membranes under radiation conditions. In this manner, it is desirable to impart the fundamental chemistry and physics learned from modeling and simulation (using descriptors associated with the analysis portion of the workflow) into mature methods and potentially numerical codes (e.g., FRD 1). Robust feedback loops that integrate experimental observation into the modeling and simulation paradigm are also needed and require an interdisciplinary approach. There is significant opportunity to approach both the simulation and experiment from a multimodal perspective that combines different types of measurement or observation. ML may be able to accelerate optimization by learning advanced, multidimensional equations that relate information at a molecular scale (obtained from either experiment or theory) to behavior at a macroscale and coarse-grain this information into new numerical models. 


\subsection{Adapt and Exploit Data Science}

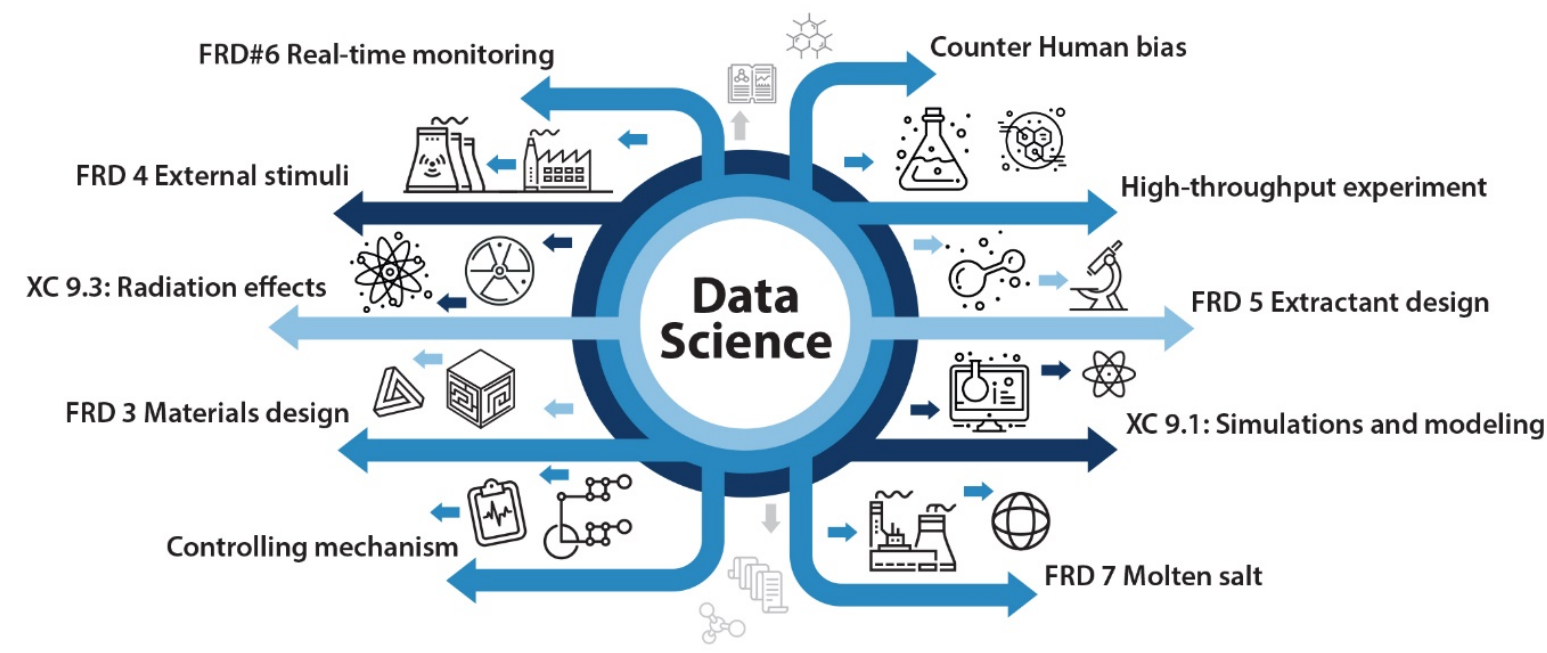

Data science is a broadly applicable and growing interdisciplinary field that incorporates statistical methods, data analysis, informatics, and related algorithms. It presents a major opportunity for making quantum leaps in technology needs identified in multiple FRDs, including real-time monitoring and design of separation agents with superior properties. Use of data science has so far been scant in research for nuclear fuel cycle separations. The major barriers lie in developing the mega databases and algorithms that will access the data and use it to predict new materials and processes. Critical capabilities needed include building reliable and accessible databases, adapting generic ML models for chemistry, and developing high-throughput experimental techniques. (Courtesy Los Alamos National Laboratory.)

Data science is playing an increasing role in our society and in scientific discovery. Data science is an interdisciplinary field that incorporates statistical methods, data analysis, informatics, and related algorithms. Its goal is to extract knowledge and underlying physics from large volumes of data. Undergoing rapid development in recent years, data science has established itself as the fourth pillar of science in addition to conventional theory, experiment, and simulation. It has revolutionized many fields such as materials science (Meredig 2019; Morgan and Jacobs 2020; Butler et al. 2018; Tao et al. 2021) and drug discovery (Lo et al. 2018; Vamathevan et al. 2019). In the last few years, data science started permeating into the non-nuclear chemical separation communities (Altintas et al. 2021) and nuclear fuel monitoring (Bae et al. 2020; Ebiwonjumi et al. 2021). However, the field is still at its nascent stage (Ferguson 2018; Gensch et al. 2021), in part because of multiple challenges related to unstructured and scarce data, and because application to soft materials requires a proper treatment of entropy and environmental interactions that are essential in many applications, including chemical separation systems.

In view of the vastness of the space of physical and chemical phenomena required to describe the nuclear fuel cycle, combining data science, modeling and simulation, and experimental measurements has the potential to transform the field by enabling real-time capabilities to curate and analyze large volumes of data, automatically adjusting data collection during operation, or countering the human bias by allowing exploration of radically different processes and extractant with high selectivity in separation, to name only a few possibilities. This augmented approach will accelerate discovery of underlying physicochemical determinants of the separation processes in complex environments across multiple time and length scales. In this crosscutting challenge, we will discuss potential applications of data science and highlight the critical technical capabilities that need to be further developed to enable applications to the nuclear fuel cycle. 


\subsubsection{Technical Challenges in Applying Data Science in Advanced Nuclear Fuel Cycle Separations}

Many research areas related to the advanced nuclear fuel cycle can directly benefit from the adoption of modern data science approaches. We list a few examples below, but this list is not exhaustive.

Counter human cognitive bias to accelerate materials and chemistry discovery

(intersects with FRDs 1, 2, 3, 4, 5, 7)

Until now, most scientific discoveries generally resulted either from exploiting a scientist's chemical and physical intuition or by serendipity. After initial discovery of a new chemistry, follow-on research typically systematically studies the key factors that impact performance and expand the chemical space, often in an incremental fashion, through slight modifications of the base chemistry. This approach is natural because it is impossible to manually exhaust the near-infinite design space for materials and chemistry, even at the level of large research teams or institutions. The downside of this approach is that it is subject to common human cognitive biases, heuristics, and social influences that all tend to "localize" further investigations in the neighborhood of previous successful examples. A recent inorganic-synthesis study demonstrates that the anthropogenic biases in chemical reactions hinder the discovery of new materials (Jia et al. 2019). This example shows that ML models trained on a randomized reaction dataset can outperform human-selected options, which highlights the importance of recognizing that human biases affect research design and indicates that modern data science can help mitigate these biases.

Despite the daunting challenges, impressive progress has been made in nuclear energy science in the last seven decades, such as identifying practical extractants for the challenging An/Ln separation needed in certain fuel cycle options. However, there is an urgent need to discover new chemistry to remove cladding (FRD 1), new materials for gas-phase separation (FRD 3), new chemistry for actinide separation (FRD 2 and 5), new control mechanisms (FRDs 4 and 7) to optimize and improve the efficiency of the nuclear cycle, and quantify and characterize the behavior of constituents in processing streams (FRD 6). Considering the astronomically large design space of these problems, it is of paramount importance to go beyond the exploitation of known chemistry and explore new unknown territories to enable revolutionary discoveries. Data science, in combination with the development of high-throughput computational and experimental capabilities, is the key to achieve this goal.

Understand the fundamental complex physics/chemistry using interpretable machine learning (intersects with all FRDs and the Radiation Effects crosscut)

Data science is particularly well suited to identify hidden relationship among complex factors, variables, parameters, and output. Although simulation can in principle provide an atomic level picture of the process, often the systems are either too large or too complicated for such simulation to be carried out with currently available computing resources. Even without such direct input, data science can intake available empirical or experimental parameters and predict observed outcomes. Using interpretable ML techniques complemented by theory and modeling, chemical insights can then be gained by identifying the key determining factors and their interactions. This is especially powerful for systems controlled by complex external stimuli that are difficult to include using conventional simulation approaches, such as various electronic, magnetic, or optical fields that can be used to improve separation efficiency (FRD 4) or simplify waste disposition. Furthermore, data science can be applied to study the intricate radiation effects highlighted in Section 9.3, particularly complex chemical reaction networks and physical property changes induced by radiation that are currently beyond the reach of direct molecular-level simulation. 
Automate in situ and real-time process monitoring in advanced nuclear fuel cycles (intersects with FRDs 3, 6, 7)

To accurately track the mass balance of SNMs, robust real-time monitoring tools are needed. Coupling of these monitoring tools to automated process control systems that are based on advanced artificial intelligence (AI) algorithms is needed to maximize plant efficiency.

As stated in Chapter 7, it is also critical to provide real-time data analysis, feedback, and decision-making support for real-time quantification and control of chemical species in nuclear processing streams. Realtime monitoring has many advantages from integration with control systems to enhance the efficiency of chemical processes to the development of next-generation surveillance systems to provide data for a comprehensive state-of-health assessment of the system (e.g., safety/reliability) that includes monitoring of radiation damage in real time. For example, advanced spectroscopic techniques can be combined with software for detection and analysis of off-gas by-products, such as iodine, for a molten salt system (Felmy et al. 2021). Furthermore, as identified in FRD 3, gas-phase separations, real-time analysis with gas analyzer or spectroscopy can provide information on the rate of vaporization at various compositions of salt, temperature, flow rates, etc., which can be used to optimize the separation process.

The key to solving these problems is the integration of in situ process monitoring with AI-based control systems. A wide variety of process monitoring tools can be leveraged. For example, the combination of optical spectroscopy with ML using data analysis tools like chemometric analysis can provide an avenue to enhance selectivity and improve the quality of characterization information. Exploring different options to integrate process-monitoring technology would support capability enhancement within advanced fuel cycles and should be a consideration in new scheme developments. Further, coupling with additional sensors and carrying out integration under AI analysis and control systems can provide robust tools to reduce the costs and timescales associated with flowsheet development and deployment. Integrated multisensor approaches providing analytical chemical and physicochemical information are of particular interest informing data science and ML/AI data interpretation.

To realize the full potential of real-time monitoring capabilities to reduce facility tuning time and down time, optimize performance, and maximize productivity, new advanced methods are needed to efficiently search large, complex parameter spaces in real time and to predict the health and failure of the processing. Automating experimental approaches with ML-based control system will drastically accelerate the exploration of vast parameter space and identify the optimal operating conditions for specific target output. One challenge in the operating process is the variation of the incoming signals. An ML-based analytic system can help in that respect by reliably separating the signal from the noise and ensure that online monitoring is interpreted properly and not just dismissed as "instrument drift."

Complement theory and simulation to accelerate discovery

(intersects with modeling and simulation crosscut and FRDs 2, 3, 5, 7)

The application of ML-methods can significantly improve the accuracy of calculation and the efficiency of high-throughput screening. For example, ML-derived empirical potentials that implicitly include manybody correction have significantly improved the accuracy of atomic simulations across the periodic table (Behler 2021). It would be important to adopt these methodologies for nuclear fuel systems. Additionally, given the complex reactive mechanisms at play in separation processes, methods that can dynamically describe bond breaking/forming and the charge transfer processes are essential. Such methods often need to be formulated at a quantum level in a way that is computationally economical compared to conventional DFT, as illustrated by a recent development in accurate semiempirical methods (Carlson 2020). ML can further facilitate the development of new methods that will enable direct simulations of more realistic systems relevant to the nuclear separations and radiolysis. 


\subsubsection{Relevant Data Science Research Needs}

Databases relevant to the nuclear fuel cycle are needed

Above all, successful applications of data science require high-quality input data. Comprehensive and validated databases for each related area are critical for discovery. Standardized data entry and complete metadata are also critical to enable data-driven discovery. This is particularly challenging for the actinide separation sciences because the experiments are often time-consuming and require specialized facilities. Furthermore, negative results are as important as positive ones for data science, as it is essentially equally important to teach the ML platform to recognize an efficient extractant as to teach it to recognize inefficient ones. However, historically, the community has mainly encouraged the publication of positive results, leaving negative results hidden in laboratory notebooks. These negative results are a gold mine for data science and, as a community, we should develop effective mechanisms to also include these unsuccessful attempts in databases. Establishing standard data format, centralizing data storage, and making the data accessible to the community are key requirements. Data management should be robust, reliable, searchable, and verifiable, and the data should be easy to deposit and retrieve. Manual processing of data to build databases is inefficient and error prone; hence, there is a need to develop ML-trained software that can scan and recognize researcher's handwriting and translate to searchable format with proper metadata input.

Biases also arise because of lack of uniform coverage of the wide chemical space, which puts ML models at risk of becoming biased. More uniform and broad coverage should be encouraged. Finally, it is important to produce multifidelity data and ML models that can handle a diverse range of accuracies, properties, and time/length scale.

\section{Adapt generic machine learning algorithms for separations in the nuclear energy cycle, including uncertainty quantification}

Generic ML algorithms can be directly used in many fields, including social sciences and physical sciences. However, there is great potential to improve the efficiency of these generic approaches by adapting them to the specific properties and chemistries used in the nuclear fuel cycle (e.g., by designing a suite of tailored input features or by exploiting intrinsic structures of the problem (Tynes et al. 2021). Appropriate uncertainty quantification is also crucial for data science, as it helps make sound decisions about the prediction of ML algorithms.

A key challenge is to integrate the diversity of the data, which includes both experimental and simulation data, as well as heterogeneous datasets that measure properties on different time and length scales. Feature vectors need to be optimized to capture complex interrelationships among variables/measured properties. Given that separation processes are dynamic, structural and dynamic descriptors should be included when training ML models.

\section{High-throughput robotic capabilities in separation science are highly demanded for actinide chemistry}

High-throughput robotic technologies have substantially evolved over the last few decades. This field has developed to the point that it can now be successfully applied toward actinide separations science, and the consequences could be transformative. Robotic methods could aid researchers in solving actinide separations problems, considering the enormous number of variables that need to be understood and optimized to solve a separations problem. The solution matrix is critical, as are the ionic strength, $\mathrm{pH}$, temperature, and composition of the aqueous solution. Successful separations often require coextractants, holdback agents, or a combination of both. Many separations involve optimizing a blend of aqueous media with an organic solvent. Systematically defining how these variables affect partitioning factors is a daunting task for an individual researcher, as the reaction space is simply too large. Although a large number of 
actinide separation studies that have been reported, there is insufficient experimental data in the literature to properly train robust ML models. Additionally, scientific reports are rarely carried out under systematic conditions; consequently, it is difficult to directly compare results from study to study in a systematic fashion. From this perspective, high-throughput robotics represents one of the most obvious avenues to acquire large enough data sets to deliver both of the aforementioned considerations. Robotics offers the potential to rapidly and accurately characterize separations parameters on a scale that is compatible with the demands of modern ML.

\subsection{Understand and Manage Radiation Effects on Materials and Processes}

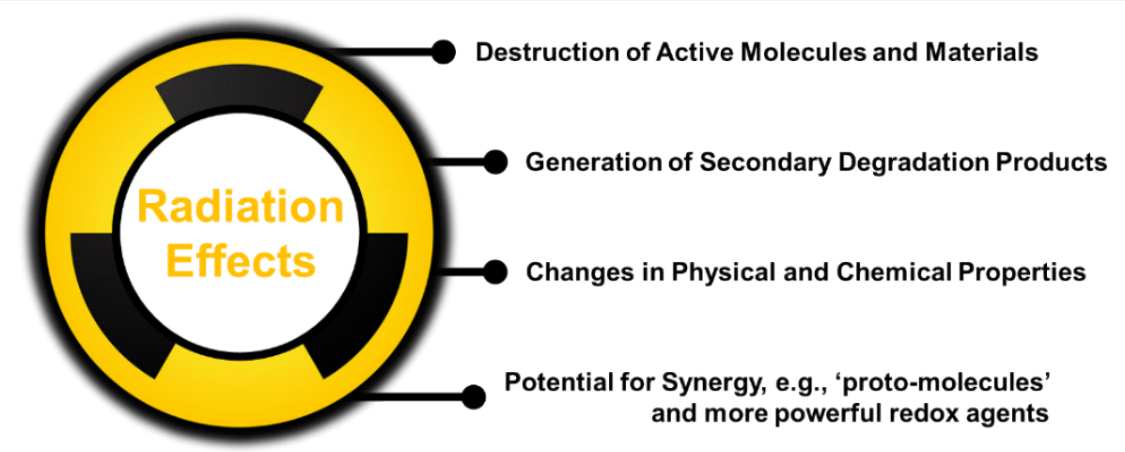

Influence of radiation effects on nuclear fuel cycle separations. (Courtesy of INL.)

Radiation effects are ubiquitous throughout all aspects of managing UNF and underpin the feasibility of separation technologies. Radiation-induced processes promote the destruction of molecules and materials with the formation of potentially detrimental degradation products and corresponding changes in physical and chemical properties, thereby influencing the effectiveness and longevity of a separations process. Although destructive effects are usually associated with radiation, beneficial effects can be foreseen. To support the accelerated development of separation technologies for advanced fuel cycles and fuels, a molecular-level understanding of radiation effects must therefore be attained over multiple time, distance, and material domains. This requires a transition from empirical measurement to accurate computer-based predictive capabilities for benchtop to plant scale by addressing the following technical challenges: (1) identifying and manipulating radiation-induced synergies for the efficient removal and treatment of coating and cladding materials in advanced reactor fuel forms; (2) evaluating the radiation robustness of materials designed for the separation of gas-phase species; (3) understanding and mastering the effects of radiation on complexants intended to simplify and compact actinide separations; (4) correlating radiation-induced changes in physical and chemical properties with the response of real-time quantification of chemical species in nuclear processing streams; and (5) integrating multiscale radiation modeling methods with validated computer models and data science approaches for accelerating the rate of reprocessing innovation.

\subsubsection{Background and Current Status}

Nuclear fission and neutron capture processes promote the conversion of nuclear fuel constituents into a complex mixture of short- and long-lived radioisotopes representing much of the periodic table. This diverse UNF mixture emits an intense radiation field capable of inducing significant chemical and physical changes in its surrounding environment. Consequently, understanding, managing, and manipulating radiation effects represents one of the greatest inherent and pervasive challenges for the development of innovative separation technologies for advanced fuel cycles.

The absorption of ionizing radiation by matter leads to the formation of a mixture of transient and steadystate radicals, ions, excited states, and molecular species, many of which are highly reactive and capable of propagating radiolysis effects. The formation of these direct and indirect radiolysis products has been shown 
to impact the efficient recovery of valuable materials from UNF and the volume of hazardous radioactive waste for final disposal. For example, in hydroreprocessing solvent extraction technologies, the direct radiolysis of water (Eq. 9.1), nitric acid (Eq. 9.2), and the aliphatic diluent (Eq. 9.3) promotes the formation of transient hydroxyl radicals $\left({ }^{\circ} \mathrm{OH}\right)$, nitrate radicals $\left(\mathrm{NO}_{3}{ }^{\circ}\right)$, and diluent organic radical cations $\left(\mathrm{RH}^{\circ+}\right)$, respectively (Mincher and Mezyk 2009):

$$
\begin{gathered}
\mathrm{H}_{2} \mathrm{O} m \mathrm{H}_{2} \mathrm{O}^{*}, \mathrm{e}_{\mathrm{aq}}{ }^{-}, \mathrm{H}^{\cdot},{ }^{\circ} \mathrm{OH}, \mathrm{H}_{2} \mathrm{O}_{2}, \mathrm{H}_{2}, \mathrm{H}_{\mathrm{aq}}{ }^{+} \\
\mathrm{HNO}_{3} m \mathrm{HNO}_{3}{ }^{*}, \mathrm{e}^{-}, \mathrm{NO}_{3}{ }^{-}, \mathrm{HNO}_{2}, \mathrm{O}^{*}, \mathrm{H}_{\mathrm{aq}}{ }^{+} \\
\mathrm{RH} m \mathrm{RH}^{*}, \mathrm{e}^{-}, \mathrm{H}^{\cdot}, \mathrm{RH}^{++}, \mathrm{R}^{\cdot}
\end{gathered}
$$

These transients exhibit significant reactivity with many of the ligands that have been proposed for separation technologies, ultimately limiting their process efficacy and longevity (Mincher et al. 2009a; Mincher et al. 2009b; Mincher et al. 2009c). Therefore, understanding the fundamental relationships between separative ligand molecular architectures and reaction mechanisms with radiolysis products would facilitate the development of computer modeling capabilities for the prediction of (1) radiation-induced changes in separation efficiency and physical properties of solvent mixtures; (2) solvent-recycle longevity; and (3) molecular architectures suitable for radiation environments. Obtaining this knowledge would support the development of chemistries that operate directly on irradiated fuel to facilitate recycling of actinides (FRD 2); application of physical phenomena, solvation, fields, and gradients for the discovery of better separations and simplified waste disposition (FRD 4); and the exploitation of coordination chemistry to simplify and compact actinide separations (FRD 5).

These radiation effects are not unique to aqueous-based reprocessing technologies, as recent pyroreprocessing-relevant research demonstrated the formation of redox active transients - the solvated electron $\left(\mathrm{e}_{\mathrm{S}}{ }^{-}\right)$and dichlorine radical anion $\left(\mathrm{Cl}_{2}{ }^{-}\right)$and the unexpected formation of Ni nanoparticles $\left(\mathrm{Ni}_{\mathrm{n}}{ }^{0}\right)$ from $\mathrm{Ni}^{2+}$ ions in molten zinc chloride $\left(\mathrm{ZnCl}_{2}\right)$ (Dias et al. 2021):

$$
\begin{gathered}
\mathrm{ZnCl}_{2} \rightsquigarrow \mathrm{Zn}^{2+}, \mathrm{e}^{-}, \mathrm{Cl}^{-} \\
\mathrm{Cl}^{-}+\mathrm{Cl}^{\cdot} \rightarrow \mathrm{Cl}_{2}{ }^{-} \\
\mathrm{Ni}^{2+} / \mathrm{Ni}^{+}+\mathrm{e}^{-} \rightarrow \mathrm{Ni}^{+} / \mathrm{Ni}^{0}
\end{gathered}
$$

A schematic for the radiation-induced evolution of $\mathrm{Ni}^{2+}$ in molten salt media is shown in Figure 9.2. These molten salt radiation effects may lead to unanticipated changes in material distribution and significant changes in corrosion potential, which would ultimately impact the efficiency of pyroprocessing technologies and the longevity of plant materials that are already in contact with a highly corrosive medium. With the recent availability of high-temperature molten salt irradiation capabilities (Philips et al. 2019), this largely unexplored pyroprocessing radiation chemistry research space is now accessible for investigation. Knowledge in this area would support redox potential/electrochemical control for the optimization/simplification of actinide separations in molten salt systems that can benefit both metal fuel electrorefining and molten salt-fueled reactors (FRD 7).

Despite significant advances over the last decade, our current knowledge of radiation effects in the area of separation technologies for advanced fuel cycles and fuels is predominantly constrained to empiricism, which is insufficient for the development of quantitative predictive computer modeling capabilities to support separations research from benchtop to process scale. To remedy this knowledge gap, a molecularlevel understanding of radiation effects must be attained over multiple time, distance, and material domains. 
This transition from empiricism to accurate predictive capabilities requires the following technical challenges to be addressed.

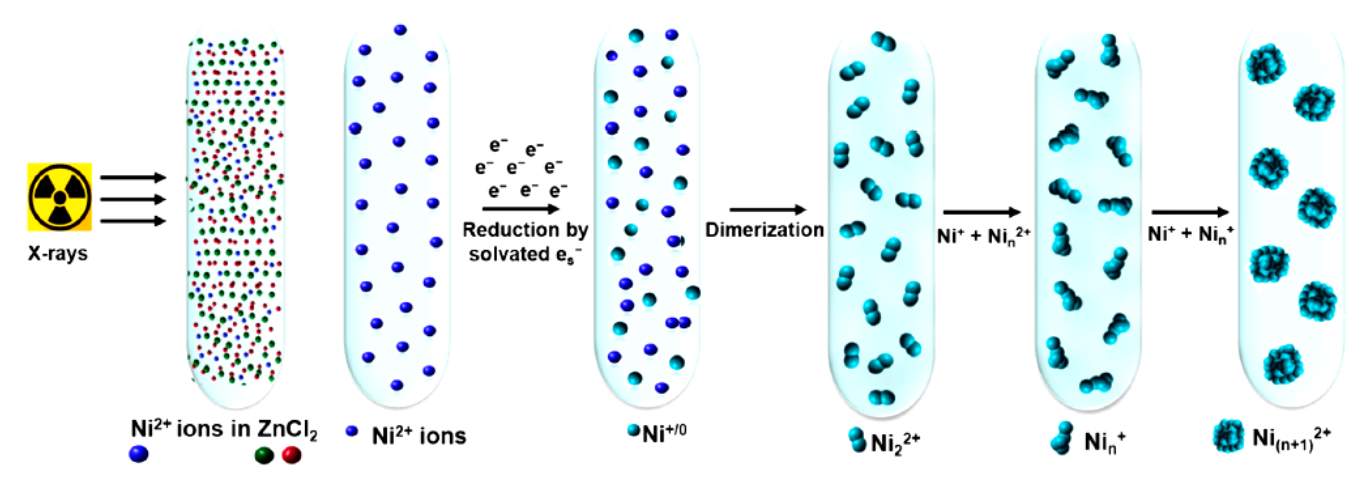

Figure 9.2. Schematic for the radiation-induced reduction of nickel cations $\left(\mathrm{Ni}^{2+} / \mathrm{Ni}^{+}\right)$to nanoparticles in molten zinc chloride $\left(\mathrm{ZnCl}_{2}\right)$ (Dias et al. 2021, reprinted with permission from American Chemical Society).

\subsubsection{Technical Challenges and Research Directions for Radiation Effects}

Identification and manipulation of radiation-induced synergy for the efficient removal and treatment of coating and cladding materials in advanced reactor fuel forms

The development of chemical methods for the removal and treatment of cladding materials is essential for increased efficiency of fuel throughput and for reducing the volume of waste through the recovery of cladding and embedded radionuclide materials. However, the selection of such methods necessitates a solid understanding of radiation effects on the chemicals and processes to be employed. In contrast to the aforementioned implications of radiation-induced destruction of process chemicals on limiting efficiency and longevity, there is also the potential for synergism. For example, irradiation of coating/cladding removal agents, such as sulfur monochloride and thionyl chloride, may promote the formation of radiolysis products that have more potent redox properties than the parent compounds (e.g., $\mathrm{SO}_{2}{ }^{*}$ and $\mathrm{Cl}^{\circ}$ ), thereby enhancing the rate of coating/cladding removal and ultimately increasing the efficiency of the cladding dissolution process. Another example of potential radiation synergy can be found in the treatment of TRISO particle fuel by sonochemistry. Sonication uses acoustic forces to disrupt materials. The collapse of cavitation bubbles in water yields physically damaging shockwaves plus significant amounts of energy that promote the formation of reactive chemical species similar to those generated by the absorption of radiation, such as the highly oxidizing ${ }^{\circ} \mathrm{OH}$ and $\mathrm{H}^{\cdot}$ atoms (Yasui et al. 2019). These radical species are likely involved in the cladding removal mechanism, and their chemistry under these conditions must be fully understood to facilitate optimal manipulation. Further, can the same radiation chemistry principles for the isolation of specific radicals (Buxton et al. 1988) be applied to sonochemistry approaches? Such knowledge may facilitate the design of removal/treatment processes that can be tailored to specific coating/cladding compositions. Consequently, there is a need for identifying, understanding, and manipulating radiation effects on chemicals and processes proposed for the efficient removal and treatment of coating and cladding materials.

Further, fuel coatings and cladding may have undergone significant radiation-induced chemical change during reactor operations, which may influence the efficacy of proposed methods to remove and treat these materials. This potentially further complicates the associated radiation chemistry. For example, in-reactor radiolysis and hydrolysis promotes the formation of (oxy)hydroxide corrosion layers on Al cladding (IAEA 2009). These Al mineral phases are expected to react very differently with coating/cladding removal/treatment agents and their radiolysis products as compared with the initial aluminum oxide coating. 
Similar scenarios can be envisioned for advanced reactor fuel forms, so a thorough understanding of the interactions between radiation-modified fuel surfaces and the chemicals and processes envisioned for their removal and treatment is necessary.

\section{Evaluation of the radiation robustness of materials designed for the separation of gas-phase species}

Aqueous reprocessing of UNF results in the emission of multiple volatile species. Of particular concern are ${ }^{129 / 131} \mathrm{I},{ }^{85} \mathrm{Kr}$, and $\mathrm{Xe}$. Iodine-129 is a highly volatile long-lived isotope (half-life $\left(t_{1 / 2}\right)=1.57 \times 10^{7}$ years, $E_{\beta}$ $=194 \mathrm{keV}$ ) that needs to be captured and reliably stored for the duration of its long decay. In contrast, ${ }^{131} \mathrm{I}$ is a volatile short-lived isotope $\left(t_{1 / 2}=8.02\right.$ days, $\left.E_{\beta}=971 \mathrm{keV}\right)$ that only needs to be stored for a few months. Recent studies on ${ }^{129 / 131}$ I capture have focused on new classes of porous sorbents, including titanosilicates, mesoporous silicas, silver-impregnated silica and alumina, silver-functionalized aerogels, chalcogels, macroreticular ion exchange resins, MOFs, and COFs, and porous organic polymers (Huve et al. 2020). However, there is very little information on the impact of radiation on the ${ }^{129 / 131}$ I adsorption performance of these new porous sorbents, and thus it must be fully investigated. Further, ${ }^{129 / 131}$ I is likely to form organic compounds such as methyl iodide, via iodination of hydrocarbons, present in the processing medium and/or gas stream. The radiation-induced chemistry of these organo-iodides is not well characterized under expected process conditions. This information is not only essential for the accountancy and disposition of ${ }^{129 / 131} \mathrm{I}$ but also in determining their efficacy of these compounds on separation process performance. Consequently, an in-depth understanding of radiation-induced ${ }^{129 / 131}$ I reaction pathways must be attained.

With regards to ${ }^{85} \mathrm{Kr}$ and $\mathrm{Xe}$, one promising new avenue is the adsorption of these gases into MOFs, which have extremely high porosity, enormous internal surface areas, and can incorporate a vast array of organic and inorganic components (Elsaidi et al. 2020). Radiation robustness studies on this class of materials have demonstrated that they are mostly radiation-resistant. However, significant crystal-structure deformation and phase changes have been observed for relatively low doses of $\sim 10 \mathrm{kGy}$. While some radiation effects work has been done, the impacts of radiation damage at higher doses, elevated temperatures, and in the presence of water (steam)/incorporated $\mathrm{CO}_{2}$ need to be fully evaluated, as they may have significant effects on the ${ }^{85} \mathrm{Kr}$ and $\mathrm{Xe}$ adsorption performance of MOFs.

\section{Understand and master the effects of radiation on complexation chemicals intended to simplify and} compact actinide separations

All potential chemical species to be employed in a separation technology need to be radiation-robust. However, there are three distinct radiation-robustness research areas that require significant attention to support the development of simplified actinide separation processes. The first area relates to effect of radiation properties (i.e., the type and energy of radiation). UNF radiation fields are multicomponent, predominantly composed of $\alpha, \beta$, and $\gamma$ radiation contributions. Most radiation-robustness studies to date have employed $\gamma$ radiation fields to study the effects of $\gamma$ and $\beta$ radiolysis. Both of these are low linearenergy-transfer radiations that promote essentially the same chemistry. In contrast, there are few studies that have investigated the impact of $\alpha$ particles on the radiation robustness of separations ligands, as this typically entails the use of either sufficiently high quantities of $\alpha$ emitter in solution or heavy ion accelerators. However, understanding the impacts of $\alpha$ radiolysis is critical, as $\alpha$ particles are high linearenergy-transfer radiation and yield very different distributions of radiolysis products. Therefore, the apparent lifetime of $\gamma / \beta$ radiation evaluated process chemicals may be significantly different in the presence of an $\alpha$ radiation field. Consequently, a quantitative understanding of $\alpha$ radiolysis is imperative for the development of accurate predictive separation process models.

The second research gap area is the impact of metal ion complexation on the radiochemical behavior of coordinating ligand complexes vs. the free/noncomplexed ligand. Most radiation-robustness studies have 
only focused on noncomplexed ligands in condensed media. However, metal-ion complexation can have drastic effects on the radiolytic susceptibility of a ligand, as demonstrated by a recent kinetic study on the reaction of $\mathrm{RH}^{\cdot+}$ with TBP, N,N-di-(2-ethylhexyl)butyramide (DEHBA), and $N, N$-di-2ethylhexylisobutryamide (DEHiBA) in which the latter two ligands exhibited enhanced reactivity upon completion to U (Celis-Barros et al. 2021). This kinetic enhancement upon metal-ion complexation may directly translate into drastically different radiochemical behaviors at steady-state timescales, which would ultimately impact the ability to predict a ligand's longevity and potentially the suite of degradation products arising from its radiolysis. Consequently, understanding the impact of metal ion complexation on radiation effects is critical for the evaluation of coordinating ligands.

The third research area is associated with the design of new coordinating molecules for advanced fuel cycles and fuels. Can radiation effects be leveraged to "switch on/off coordination functionality? This novel concept would require the design of so-called proto-molecules, which when exposed to ionizing radiation undergo tailored chemical transformations that result in a desired change in coordination properties, thereby facilitating selective extraction and/or recovery of elements based on absorbed dose. Although these protomolecules would be single use, they may provide a new approach to simplify and compact actinide separations through having a single coordinating chemical species that progressively adapts to the different stages of a separations process by leveraging available radiation fields and the above knowledge of radiation quality and complexation effects.

\section{Correlate radiation-induced changes in physical and chemical properties with the response of real-time quantification of chemical species in nuclear processing streams}

Radiation can have two distinct effects on the real-time quantification of chemical species. The first is the general damaging and destruction of materials used in quantification technologies. For example, fiber-optic materials for optical spectroscopy progressively darken with absorbed radiation dose. These radiationinduced defects typically result in the loss of short wavelength sensitivity, as shown in Figure 9.3, which could directly impair the accurate measurement of specific nuclear materials in separation process streams.

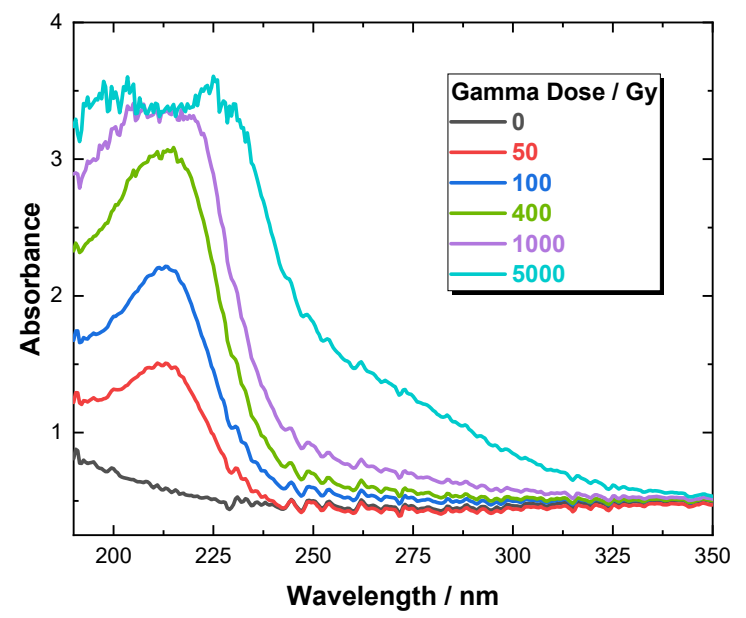

Figure 9.3. Change in the short-wavelength absorption properties of fiber optics with absorbed $\mathrm{y}$ dose $\left(47.4 \mathrm{~Gy}^{\mathrm{min}}{ }^{-}\right.$ $\left.{ }^{1}\right)$ performed at the INL Center for Radiation Chemistry Research. (Courtesy of INL.)

Further, some materials used in these quantification technologies may come into contact with separation media, and thus their integrity is brought into question, especially as these materials are likely benchmarked against chemicals not representative of those generated radiolytically. Consequently, the selection of 
materials and their configurations must be quantitatively evaluated to determine their radiation robustness, reliability, and longevity.

The second radiation effect of concern to the real-time quantification of chemical species relates to the ability of a monitoring technique to account for radiation-induced changes in the physical and chemical properties of the separations medium. For example, fluid bubblers rely on known fluid densities to calculate volumes, but irradiation of hydroreprocessing media can promote changes in a solvent system's density, viscosity, and surface tension (Tripathi and Ramanujam 2003). Further, electrochemical sensors use a process solution's electrochemical response, which if not "trained" to account for radiation-induced chemical changes to a solution's composition, may provide false positive/negative information on nuclear material accountancy. Therefore, correlations between the response of a given real-time monitoring technique and radiation-induced changes to a processing medium must be developed.

Integrate radiation multiscale modeling methods with computation and data-science approaches for accelerating the rate of innovation

Fundamental models exist for the description of radiation chemical effects in a variety of materials, but no such model has been developed to give a holistic prediction of chemistry throughout a whole separations process. Such a capability would rapidly accelerate the development of advanced separation technologies. Although individual modeling and simulation codes exist for individual parts of a separation process (e.g., the Argonne Model for Universal Solvent Extraction [AMUSE], Frey et al. 2012), these often lack a quantitative radiation effects component, thereby significantly limiting their applied utility. Consequently, radiation chemistry modules need to be developed for hydro- and pyroprocessing. This would necessitate extensive research into the basic radiation reaction networks of organic, ionic-liquid, mixed-phase (i.e., aqueous and organic combined), and molten-salt media to cover all proposed avenues for advanced fuel cycle separations processes. These endeavors would incorporate the development of big data-science approaches to interrogate and connect the wealth of experimental data necessary to develop these models. Once developed and evaluated, these radiation modules must then be integrated with other chemistry and engineering modeling and simulation modules to yield the desired holistic separations model.

\subsection{References}

Altintas, C.; Altundal, O. F.; Keskin, S.; Yildirim, R. Machine Learning Meets with Metal Organic Frameworks for Gas Storage and Separation. Journal of Chemical Information and Modeling 2021, 61, 2131-46.

Bae, J. W.; Rykhlevskii, A.; Chee, G.; Huff, K. D. Deep Learning Approach to Nuclear Fuel Transmutation in a Fuel Cycle Simulator. Annals of Nuclear Energy 2020, 139 [107230]. https://doi.org/10.1016/j.anucene.2019.107230.

Behler, J. Four Generations of High-Dimensional Neural Network Potentials. Chemical Reviews 2021, $121,10037-72$.

Butler, K. T.; Davis, D. W.; Cartwright, H.; Isayev, O.; Walsh, A. Machine Learning for Molecular and Materials Science. Nature 2018, 559, 547-55.

Carlson, R. K.; Cawkwell, M. J.; Batista, E. R.; Yang, P. Tight-Binding Modeling of Uranium in an Aqueous Environment. Journal of Chemical Theory and Computation 2020, 16, 3073-83.

Celis-Barros, Pilgrim, C. D.; C.; Cook, A. R.; Grimes, T. S.; Mezyk, S. P. Horne, G. P. Influence of Uranyl Complexation on the Reaction Kinetics of the Dodecane Radical Cation with Used Nuclear Fuel Extraction Ligands (TBP, DEHBA, and DEHiBA). Physical Chemistry and Chemical Physics 2021, 23, 24589-97. https://doi.org/10.1039/D1CP03797H. 
Clark, A. E.; Adams, H.; Hernandez, R.; Krylov, A. I.; Niklasson, A. M. N.; Sarupria, S.; Wang, Y.; Wild, S. M.; Yang, Q. The Middle Science: Traversing Scale In Complex Many-Body Systems, ACS Central Science 2021, 7, 1271-87.

Dias, E. T.; Gill, S. K.; Liu, Y.; Halstenberg, P.; Dai, S.; Huang, J.; Mausz, J.; Gakhar, R.; Phillips, W. C.; Mahurin, S.; Pimblott, S. M.; Wishart, J. F.; Frenkel, A. I. Radiation-Assisted Formation of Metal Nanoparticles in Molten Salts. Journal of Physical Chemistry Letters 2021, 12, 157-64. https://dx.doi.org/10.1021/acs.jpclett.0c03231.

DOE-SC. 2016. Basic Research Needs for Synthesis Science, US Department of Energy, Office of Science, https://www.osti.gov/servlets/purl/1616513.

Ebiwonjumi, B.; Cherezov, A.; Dzianisau, S.; Lee, D. Machine Learning of LWR Spent Nuclear Fuel Assembly Decay Heat Measurements. Nuclear Engineering and Technology 2021, 53, 3563-79.

Elsaidi, S. K.; Mohamed, M. H.; Helal, A. S.; Galanek, M.; Pham, T.; Suepaul, S.; Space, B.; Hopkinson, D.; Thallapally, P. K.; Li, J. Radiation-Resistant Metal-Organic Framework Enables Efficient Separation of Krypton Fission Gas From Spent Nuclear Fuel. Nature Communications 2020, 11(3103). https://doi.org/10.1038/s41467-020-16647-1.

Felmy, H. M.; Clifford, A. J.; Medina, A. S.; Cox, R. M.; Wilson, J. M.; Lines, A. M.; Bryan, S. A. OnLine Monitoring of Gas-Phase Molecular Iodine Using Raman and Fluorescence Spectroscopy Paired with Chemometric Analysis. Environmental Science and Technology 2021, 55(6), 38983908. https://doi.org/10.1021/acs.est.0c06137.

Ferguson, A. L. Machine Learning and Data Science in Soft Materials Engineering. Journal of Physics: Condensed Matter 2018, 30(4), 043002. https://iopscience.iop.org/article/10.1088/1361$648 X / a a 98 b d$.

Frey, K.; Krebs, J. F.; Pereira, C. Time-Dependent Implementation of Argonne's Model for Universal Solvent. Extraction. Industrial and Engineering Chemistry Research 2012, 51, 13219-26. https://doi.org/10.1021/ie301421d.

Gensch, T.; Gomes, G. P.; Friederich, P.; Peters, E.; Gaudin, T.; Pollic, R.; Jorner, K.; Nigam, A.; Lindner-D’Addario, M.; Sigman, M. A.; Aspuru-Guzik, A. A Comprehensive Discovery Platform for Organophosphorus Ligands for Catalysis. Journal of the American Chemical Society 2022, 144(3), 1205-17. https://doi.org/10.1021/jacs.1c09718

Goings, J. J.; Lestrange, P. J.; Li, X. Real-Time Time-Dependent Electronic Structure Theory. WIREs Computational Molecular Science 2018, 8(1), e1341. https://doi.org/10.1002/wcms.1341.

Huve, J.; Ryzhikov, A.; Nouali, H.; Lalia, V.; Auge, G.; Jean Daou, T. Porous Sorbents for the Capture of Radioactive Iodine Compounds: A Review. RSC Advances 2018, 8, 29248-73. https://doi.org/10.1039/c8ra04775h.

IAEA. Corrosion of Research Reactor Aluminium Clad Spent Nuclear Fuel in Water. IAEA-TECDOC1637, International Atomic Energy Agency, Vienna, 2009.

Jia, X.; Lynch, A.; Huang, Y.; Danielson, M.; Lang'at, I.; Milder, A.; Ruby, A. E.; Wang, H.; Friedler, S. A.; Norquist, A. J.; Schrier, J. Anthropogenic Biases in Chemical Reaction Data Hinder Exploratory Inorganic Synthesis. Nature 2019, 573, 251-55.

Lo, Y.; Rensi, S. E.; Torng, W.; Altman, R. B. Machine Learning in Chemoinformatics and Drug Discovery. Drug Discovery Today 2018, 23, 1538-46.

Lu, L.; Wildman, A.; Jenkins, A. J.; Young, L.; Clark, A. E.; Li, X. The "Hole" Story in Ionized Water from the Perspective of Ehrenfest Dynamics, Journal of Physical Chemistry Letters, 2020, 11, 9946-9951. 
Meredig, B. Five High-Impact Research Areas in Machine Learning for Materials Science. Chemistry of Materials 2019, 31, 9579-81.

Mincher, B. J.; Mezyk, S. P. Radiation Chemical Effects on Radiochemistry: A Review of Examples Important to Nuclear Power. Radiochim. Acta 2009, 97, 519-34. https://doi.org/10.1524/ract.2009.1646.

Mincher, B. J.; Modolo, G.; Mezyk, S. P. Review Article: The Effects of Radiation Chemistry on Solvent Extraction: 1. Conditions in Acidic Solution and a Review of TBP Radiolysis. Solvent Extraction and Ion Exchange 2009a, 27(1), 1-25. https://doi.org/10.1080/07366290802544767.

Mincher, B. J.; Modolo, G.; Mezyk, S. P. Review Article: The Effects of Radiation Chemistry on Solvent Extraction: 2. A Review of Fission-Product Extraction. Solvent Extraction and Ion Exchange 2009b, 27(3) 331-53. https://doi.org/10.1080/07366290902821263.

Mincher, B. J.; Modolo, G.; Mezyk, S. P. Review Article: The Effects of Radiation Chemistry on Solvent Extraction 3: A Review of Actinide and Lanthanide Extraction. Solvent Extraction and Ion Exchange 2009c, 27(5-6), 579-606. https://doi.org/10.1080/07366290903114098.

Morgan, D.; Jacobs, R. Opportunities and Challenges for Machine Learning in Material Science. Annual Review of Materials Research 2020, 50, 71-103.

NAS. 2019. A Research Agenda for Transforming Separation Science, National Academy of Sciences, https://www.nap.edu/catalog/25421/a-research-agenda-for-transforming-separation-science.

Phillips, W. C.; Gakhar, R.; Horne, G. P.; Layne, B.; Iwamatsu, K.; Ramos-Ballesteros, A.; Shaltry, M. R.; LaVerne, J. A.; Pimblott, S. M.; Wishart, J. F. Design and Performance of HighTemperature Furnace and Cell Holder for In Situ Spectroscopic, Electrochemical, and Radiolytic Investigations of Molten Salts. AIP Review of Scientific Instruments, 2020, 91(8), 083105. https://doi.org/10.1063/1.5140463.

Tao, H.; Wu, T.; Aldeghi, M.; Wu, T. C.; Aspuru-Guzik, A.; Kumacheva, E. Nanoparticle Synthesis Assisted by Machine Learning. Nature Reviews Materials 2021, 6, 701-16.

Tripathi, S.C.; Ramanujam, A. Effect of Radiation-Induced Physicochemical Transformations on Density and Viscosity of $30 \% \mathrm{TBP}-n$-Dodecane- $\mathrm{HNO}_{3}$ System. Separation Science and Technology 2003, 38, 2307-26. https://doi.org/10.1081/SS-120021626.

Tynes, M.; Gao, W.; Burrill, D. J.; Batista, E. R.; Perez, D.; Yang, P.; Lubbers, N. Pairwise Difference Regression: A Machine Learning Meta-Algorithms for Improved Prediction and Uncertainty Quantification in Chemical Search. Journal of Chemical Information and Modeling 2021, 61, 3846-3857.

Vamathevan, J.; Clark, D.; Czodrowski, P.; Dunham, I.; Ferran, E.; Lee, G.; Li, B.; Madabhushi, A.; Shah, P.; Spitzer, M.; Zhao, S. Applications of Machine Learning in Drug Discovery and Development. Nature Reviews Drug Discovery 2019, 18, 463-477.

Xie, T.; Grossman, J. C. Crystal Graph Convolutional Neural Networks for an Accurate and Interpretable Prediction of Material Properties. Physical Review Letters 2018, 120,145301. https://doi.org/10.1103/PhysRevLett.120.145301.

Yasui, K.; Tuziuti, T.; Kanematsu, W. Mechanism of OH Radical Production from Ozone Bubbles in Water after Stopping Cavitation. Ultrasonics Sonochemistry 2019, 58, 104707. https://doi.org/10.1016/j.ultsonch.2019.104707. 


\section{Conclusions and Perspectives}

The emerging technologies and future research directions (FRDs) identified here along with three crosscutting research topics aim to solve separation problems that will enable safe, secure, economical, and environmentally responsible recycling of actinides needed for advanced fuel cycle options (Figure 10.1). As the demand for economical clean energy grows more acute to achieve domestic and global decarbonization targets, pursuit of the research goals described in this report will more precisely inform decision makers of the potential benefits of the available fuel cycle options. Given the uncertainty in choices among advanced reactor designs and whether and how to accomplish fuel recycle for existing and future used nuclear fuels, a toolbox approach must be taken in the development of advanced fuel cycle separations, and this approach is reflected in this report. Accordingly, the supporting research has been anticipated and elaborated in detail with selected references serving as a guide to the applicable literature.

\section{ACTINIDE RECYCLE}

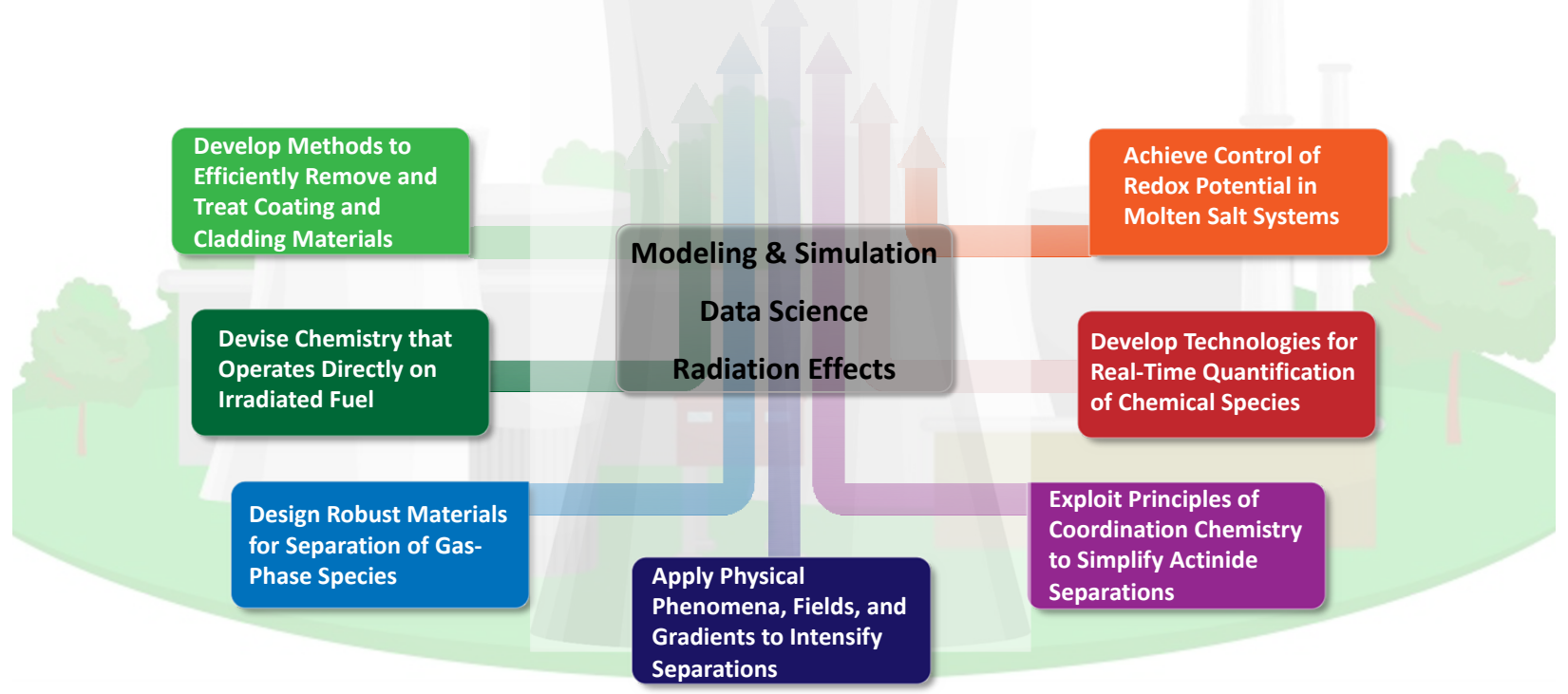

Figure 10.1. Future research directions supporting the goal of actinide recycle for sustainable nuclear energy draw from crosscutting research on modeling and simulation, data science, and minimizing radiation effects. (Courtesy of ORNL.)

Fuel recycle rests on the foundation of separations. Except for molten-salt reactor fuel, irradiated fuel must first be exposed, which requires disassembling hardware and breaching hardy fuel coatings or claddings. This requires highly energetic mechanical and chemical techniques. Once exposed, the irradiated fuel in its many possible forms then can be subjected to physical and chemical processes involving combinations of separation agents and inputs of energy in various forms. Process streams of separated material will include actinides, tailored for subsequent fuel fabrication and recycle, and residuals, adapted for incorporation into waste forms for disposal. Given the large number and variety of components and extreme hazard involved, the overall separation problem is exceedingly complex, and the task to simplify it is equally so. Using present-day technologies, developed separation methods capable of supporting fuel-cycle options would be complex, large, and costly to implement. The need for research is widely acknowledged to develop altogether new technologies, overcome scale-up difficulties in emerging technologies, and improve upon mature technologies. To address these challenges, the workshop approached nuclear separations generally, organizing discussions around the types of phases that would actively undergo mass transfer in applicable 
separation processes: gas-solid, liquid-solid, and liquid-liquid. Although somewhat arbitrary, this division was surprisingly successful at sorting separation techniques at various stages of irradiated fuel processing. Ordered roughly according to the sequence of the treatment train, the following FRDs emerged:

FRD 1: Develop Methods to Efficiently Remove and Treat Coating and Cladding Materials

FRD 2: Devise Chemistry that Operates Directly on Irradiated Fuel

FRD 3: Design Robust Materials for Separation of Gas-Phase Species

FRD 4: Apply Physical Phenomena, Fields, and Gradients to Intensify Separations

FRD 5: Exploit Principles of Coordination Chemistry to Simplify Actinide Separations

FRD 6: Develop Technologies for Real-Time Quantification of Chemical Species

FRD 7: Achieve Control of Redox Potential in Molten Salt Systems

Crosscutting research directions arose to address common themes across the FRDs. Discussions in all three panels included elements of modeling and simulation to accelerate research, and data science incorporating machine learning emerged as a growing opportunity. The development of almost any technology for fuel cycle separations incurs evaluating and managing radiation effects, possibly even exploiting them. Accordingly, the following crosscutting topics were identified:

Crosscut 1: Accelerate the Rate of Innovation by Leveraging Simulation and Modeling

Crosscut 2: Adapt and Exploit Data Science

Crosscut 3: Understand and Manage Radiation Effects on Materials and Processes

Comparison of the present report with earlier related DOE workshop reports over the past several decades (Figure 10.2) provides insight into the roles of SS\&T in nuclear fuel cycles and evolution of corresponding research needs within DOE. Among the reports, the present workshop report dwells more squarely in the domain of applied research while using the tools of, and drawing inspiration from, fundamental science. In so doing, it focuses more directly on separations research needs projected to benefit DOE-NE's Office of Materials and Chemical Technologies. Two earlier reports (Finck 2005; Roberto 2006) sponsored by the DOE Office of Science presented needs for basic research with emphasis on SS\&T. With active participation by DOE-NE, the former, entitled The Path to Sustainable Nuclear Energy. Basic and Applied Research Opportunities for Advanced Fuel Cycles, more widely encompassed reactors, fuels, separations, and waste forms. ${ }^{5}$ Research needs were identified in the areas of detectors, materials, and advanced processes having obvious commonalities with the present report. This report also noted the need for "translational tools," referring to how to bridge the gap between basic science and technology development by breaking out of Edisonian approaches. The latter Basic Energy Sciences Workshop on Basic Research Needs for Advanced Nuclear Systems, often called the ANES report, identified scientific grand challenges along with a set of priority research directions for fundamental research that may be of interest here for comparison to our FRDs:

- Nanoscale design of materials and interfaces that radically extend performance limits in extreme radiation environments

- Physics and chemistry of actinide-bearing materials and the $f$-electron challenge

- Microstructure and property stability under extreme conditions

- Mastering actinide and fission product chemistry under all chemical conditions

- Exploiting organization to achieve selectivity at multiple length scales

- Adaptive material-environment interfaces for extreme chemical conditions

${ }^{5}$ A 2017 workshop report entitled Basic Research Needs for Future Nuclear Energy was almost exclusively focused on reactors (Beierschmitt 2017). 
- Fundamental effects of radiation and radiolysis in chemical processes

- Fundamental thermodynamics and kinetic processes in multicomponent systems for fuel fabrication and performance

- Predictive multiscale modeling of materials and chemical phenomena in multicomponent systems under extreme conditions

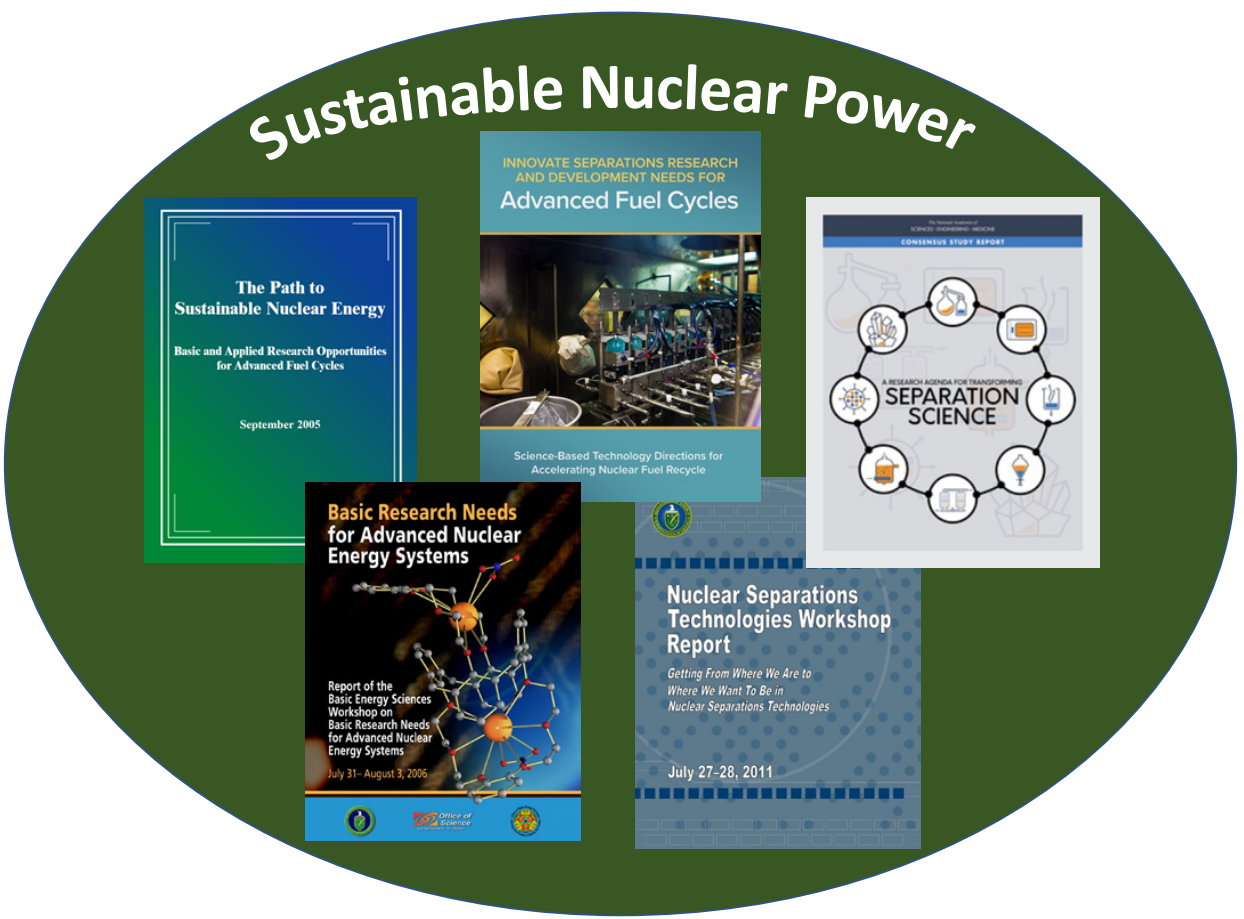

Figure 10.2. Notable workshop reports dealing with research needs in SS\&T relevant to nuclear fuel cycles. (The cover image for A Research Agenda for Transforming Separation Science is courtesy of the National Academy of Sciences.)As characteristic of fundamental research, the needs identified in the ANES report are general and enduring. All of the above priority research directions identified in that previous report are still relevant to the FRDs and crosscutting topics described in the present report. Today's tools have grown much more powerful as assisted by DOE user facilities, and computational techniques have become much more integrated into research. This trend can only be expected to continue, as the ability to computationally design and predict the properties of materials, separation agents, and processes not only grows in its supporting role but also becomes competitive with - or even surpasses - experimental science. The in-growth of the field of data science is a powerful new research opportunity identified here.

A successor workshop to ANES held in 2011 produced a long applications-focused report (US Department of Energy 2011) entitled Nuclear Separations Technologies Workshop Report. Getting from Where We Are to Where We Want to Be in Nuclear Separations Technologies. It was sponsored by three mission-oriented DOE offices: Office of Nuclear Energy, Office of Environmental Management, and National Nuclear Security Administration, with participation from the Office of Science. The workshop raised many of the familiar themes from its predecessors that motivate the present FRDs regarding actinide separations and design of selective separation agents. However, it explicitly called out the problems of scale-up and the coupling of separations to waste form fabrication:

- Chemistry and speciation of the actinides and key fission products (Cs, Tc, I)

- Design of molecules and materials with selective separation properties

- Scale-up of separation processes from bench-top to plant 
- Interface issues between separations and waste forms/fuel fabrication

Computational modeling and simulation again appeared as an important crosscutting need. Exceptions include decorporation agents, omitted here because they are not within the scope of the DOE Office of Materials and Chemical Technologies program, and Cs and Sr separations, which have long been more of interest to the DOE Office of Environmental Management (National Research Council 2001). Although the heat emitters ${ }^{137} \mathrm{Cs}$ and ${ }^{90} \mathrm{Sr}$ (and their daughters) with approximately 30 -year half-lives play an important role in management of the fission-product waste streams in fuel cycles, their separation is no longer seen as economically warranted as a separate unit operation. The reader is directed to the National Research Council Report (2001) for an overview of separations research needs for high-level waste; in the time since this report was issued, DOE has addressed its immediate needs and has adopted technologies for implementation. A novel suggestion from the 2011 workshop is the notion of a Center of Knowledge for Nuclear Separations Technologies. Recognizing the integral importance of SS\&T to the success of multiple DOE missions, the report authors argued that the proposed center would fulfill a coordination role across DOE offices, set up a user facility for demonstration and scale-up, and supply resources to build the supporting workforce.

Most recently, the National Academy of Science (2019) provided a broad perspective on research needs across the spectrum of any conceivable SS\&T application including nuclear applications in its report entitled A Research Agenda for Transforming Separation Science. The scientific challenges identified included:

- Selectivity, capacity, and throughput

- Understanding temporal changes that occur in separation systems

- Defining standard systems, samples, and methods

- Accelerating chemical separations with data science

In the first challenge, the same needs related to thermodynamics, interfacial phenomena, and external forces were addressed as found, for example, in FRDs 4 and 7. Synthesis of separation agents and materials was strongly called out as a need for research in itself, relating to FRDs 3 and 5. In the second challenge, needs related to the stability of separation agents and materials were identified, which is an important issue as in Crosscut 3 on radiation effects. The crosscutting research topic of standards, samples, and methods in the NAS report resonates with FRD 6. NAS identified data science and modeling and simulation as crosscutting in much the same way as Crosscuts 1 and 2 herein.

In historical context, the FRDs and crosscutting topics depicted in Figure 10.1 reflect the co-evolution of radiochemistry, nuclear technologies, and SS\&T over the last century. The interdependence and intertwining of SS\&T with these fields is astonishing. How could our founding scientists such as Madame Curie and Glenn T. Seaborg have succeeded without separations chemistry? Building on their early foundations, legions of scientists and engineers have developed the field of SS\&T to enable nuclear energy. The US national laboratories can in fact trace their early mission in many respects to the development of neutron science alongside SS\&T. This initial pairing persists today in recognizable ways, as emphasized by the contributions of the cited workshop reports (Figure 10.2).

Not only has SS\&T become indispensable for nuclear fuel cycles, but it has also broadly diffused into the fabric of DOE's programs. As mentioned above, it has proven essential for environmental management, waste treatment, and national security. New or reinvigorated pursuits by DOE in critical materials, desalination, and isotope production also rely principally on SS\&T to accomplish their goals. In some respects, the diffuseness of SS\&T across DOE's programs, as well as across science and industry more broadly (National Academies of Sciences 2019), has led to difficulties in managing or even identifying SS\&T as a discipline. Realizing the integral importance of SS\&T within its energy mission, DOE has thus 
systematically promoted the cohesiveness of SS\&T across many offices, including the offices of Nuclear Energy, Environmental Management, and Basic Energy Sciences, by (1) supporting research programs focused on separations, (2) spinning off technical meetings such as the Symposia for Separation Science and Technology for Energy Applications (biennial) and the Actinide Separations Conference (annual); and (3) sponsoring research-needs workshops and commissioning corresponding reports (viz. Figure 10.2).

The present workshop and report have sought to provide the key research needs of nuclear fuel recycle. Supporting this overarching goal, FRDs and crosscutting topics identified here assimilate the conclusions and trends of multiple prior workshops approaching SS\&T from different perspectives together with new input from a diverse cross section of DOE's research community. Nuclear fuel recycle is growing even more challenging with development of advanced reactors and hardy fuels that can withstand higher burnup. Transformational separations research can change the future landscape of fuel recycle by improving upon or even enabling new options that lower costs while ensuring safety, proliferation resistance, and environmental stewardship. This report, reflecting the scientific input of the technical experts in the field, lays out the technological challenges and solutions grounded in SS\&T that will meet our nation's energy supply, environmental, and energy security needs.

\subsection{References}

Beierschmitt, K.; Buchanan, M.; Clark, A.; Robertson, I.; Britt, P.; Navrotsky, A.; Burns, P.; Tortorelli, P.; Misra, A.; Wishart, J.; Fenter, P.; Gewirth, A.; Wirth, B.; Mincher, B.; Szlufarska, I.; Busby, J.; Horton, L.; Garrett, B.; Vetrano, J. Wilk, P.; Runkles, K.; Kung, S.; Lesica, S. Basic Research Needs for Future Nuclear Energy: Report of the Basic Energy Sciences Workshop for Future Nuclear Energy, August 9-11, 2017. US Department of Energy: Washington, DC, 2017. doi:10.2172/1616270.

Finck, P.; Edelstein, N.; Allen, T.; Burns, C.; Chadwick, M.; Corradini, M.; Dixon, D.; Goff, M.; Laidler, J.; McCarthy, K.; Moyer, B.; Nash, K.; Navrotsky, A.; Oblozinsky, P.; Pasamehmetoglu, K.; Peterson, P.; Sackett, J.; Sickafus, K. E.; Tulenko, J.; Weber, W.; Morss, L.; Henry, G. The Path to Sustainable Nuclear Energy. Basic and Applied Research Opportunities for Advanced Fuel Cycles. 2005. doi:10.2172/899134.

National Academies of Sciences, Engineering, and Medicine. A Research Agenda for Transforming Separation Science. Washington, DC: National Academies Press, 2019. https://doi.org/10.17226/25421.

National Research Council. Research Needs for High-Level Waste Stored in Tanks and Bins at U.S. Department of Energy Sites: Environmental Management Science Program. Washington, DC: National Academies Press, 2001. https://doi.org/10.17226/10191.

Roberto, J.; Diaz de la Rubia, T.; Gibala, R.; Zinkle, S.; Miller, J. R.; Pimblott, S.; Burns, C.; Raymond, K.; Grimes, R.; Pasamehmetoglu, K.; Clark, S.; Ewing, R.; Wagner, A.; Yip, S.; Buchanan, M.; Crabtree, G.; Hemminger, J.; Poate, J.; Miller, J. C.; Edelstein, N.; Fitzsimmons, T.; Gruzalski, G.; Michaels, G.; Morss, L.; Peters, M.; Talamini, K. Basic Research Needs for Advanced Nuclear Systems. Report of the Basic Energy Sciences Workshop on Basic Research Needs for Advanced Nuclear Energy Systems, July 31-August 3, 2006. 2006. doi:10.2172/899045.

US Department of Energy. Nuclear Separations Technologies Workshop Report. Getting from Where We Are to Where We Want to Be in Nuclear Separations Technologies. July 27-28, 2011. https://www.energy.gov/ne/articles/nuclear-separations-technologies-workshop-report. 


\section{Acknowledgments}

This report and its corresponding workshop entitled "Innovative Separations R\&D Needs for Advanced Fuel Cycles" were funded by the Office of Materials and Chemical Technologies, Office of Nuclear Energy, US Department of Energy.

We thank the following people for key contributions to the workshop and report:

Philip Wilk and Dan Matuszak (DOE Office of Science) for their suggestions and engagement, resulting in the participation of a cohort of fundamental researchers in the workshop.

Phil Britt (ORNL), Stephen Kung (DOE-NE), Tatiana Levitskaia (PNNL), Leigh Martin (ORNL), Dean Peterman (INL), Jenifer Shafer (ARPA-E), and Terry Todd (INL, retired) for their review of the report and their helpful suggestions.

Valinda Hubbs (ORNL) for coordinating administrative matters in setting up the workshop and processing the report.

Laura Chavez-Becker (ORNL) for developing the workshop website.

Ron Kennedy and John Bui (ORNL) for setting up the Zoom format for the virtual meeting and ensuring its smooth flow.

Emily Huckabay (ORNL) for technical editing of the report and Kathy Jones (ORNL) for formatting support.

Colby Earles (ORNL) and Laddy Fields (ORNL) for graphic design.

Nathan Bessen (ORNL) for putting together the acronym table.

Plenary speakers Andrew Griffith (DOE-NE), Gregg Lumetta (PNNL), John Vienna (PNNL), Robin Taylor (National Nuclear Laboratory, UK), Michael Simpson (University of Utah), and Jenifer Shafer (Colorado School of Mines and DOE ARPA-E), whose lectures added a wealth of background material and foundation for the workshop discussions.

Workshop participants (Appendix E) for their enthusiastic comments, questions, and engagement throughout the workshop.

White paper authors (Appendix G) for volunteering their forward-looking ideas that ultimately provided a foundation for this report.

Chapter authors and co-authors (Appendix $\mathrm{H}$ ) for contributing the text for this report. 


\section{Appendix A. Composition of Irradiated Fuel}

Nuclear reactors produce sustained chain reactions of neutron-induced fission in $\mathrm{U}$ or $\mathrm{Pu}$ isotopes, creating an array of nearly 50 other elements as FPs or through radioactive decay after neutron capture reactions. Some of the key factors that determine the precise distribution of elements in irradiated fuel include the type and design of the nuclear reactor, the neutron flux as a function of energy, the identity and concentration of nuclides initially in the fuel, the possible addition of neutron absorbers (burnable poisons) to the fuel, the length of irradiation, and the amount of time that has elapsed since the fuel was removed from the reactor.

While the exact composition of irradiated fuels varies, low-enriched uranium (LEU) dioxide fuels used for power production in the United States provide an informative baseline for fuel compositions. Irradiated LEU is principally composed of unreacted $U$, which is slightly enriched in ${ }^{235} \mathrm{U}$ when the fuel is removed from the reactor (Figure A.1). On either a molar or mass basis, the remainder of the fuel is primarily transition metal $(2.8 \mathrm{~mol} \%)$ and rare earth FPs $(1.7 \mathrm{~mol} \%)$. The most abundant transition metals in irradiated fuel are $\mathrm{Zr}, \mathrm{Mo}, \mathrm{Ru}$, and Pd, while the most abundant rare earth FPs are the lanthanides from La through Sm, with lesser amounts of Eu and Gd. In some fuels, substantial amounts of Gd or Er oxides also are mixed with the fuel as burnable poisons, which can increase their concentrations by several mol\% over the reference case shown in Figure A.1. In addition, noble gases ( $\mathrm{Kr}$ and $\mathrm{Xe}$ ) and transuranic (TRU) elements each constitute approximately $1 \mathrm{~mol} \%$ of the irradiated fuel.

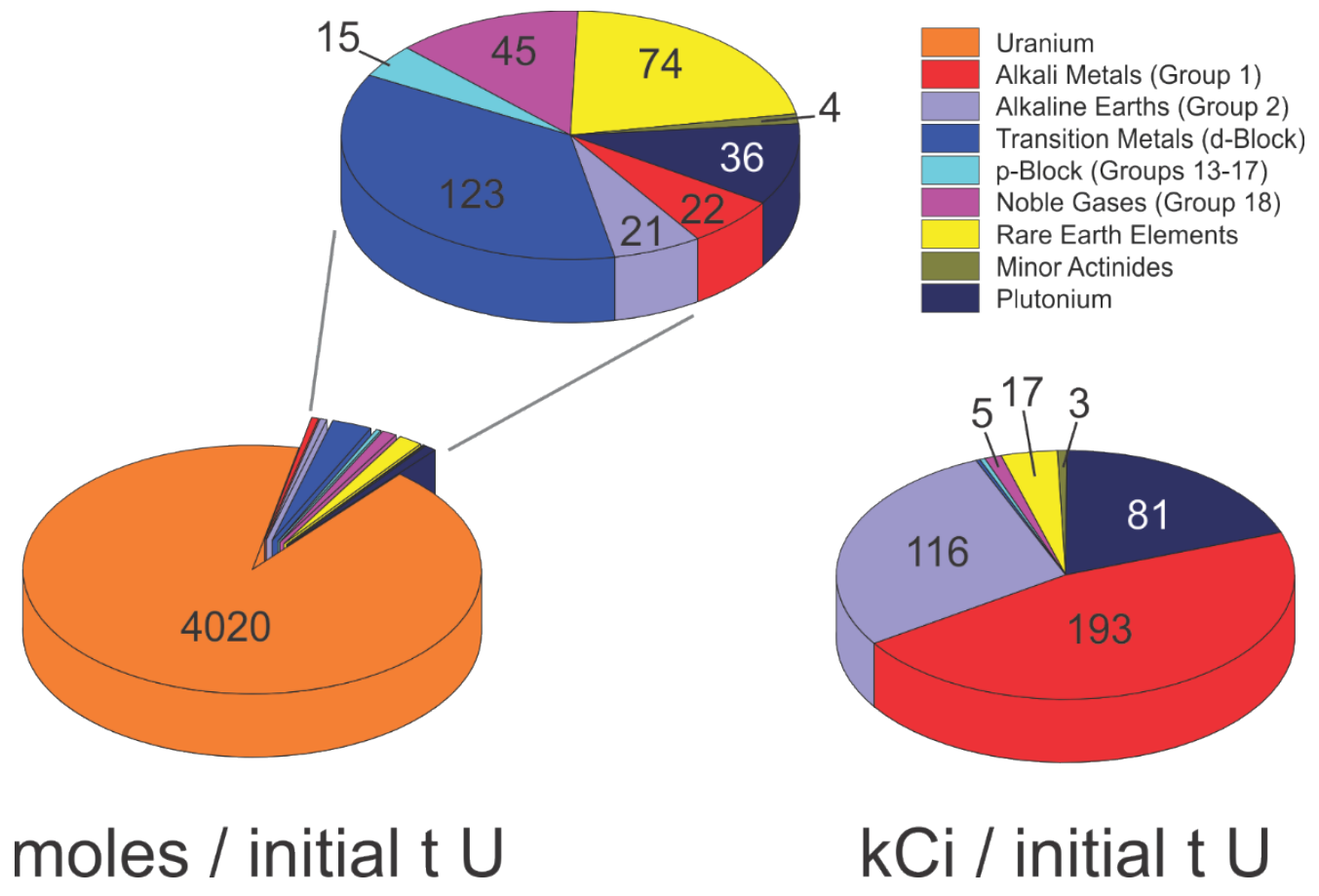

Figure A.1. Postirradiation composition of 1 ton $3.2 \%$ enriched $\mathrm{UO}_{2}$ fuel 10 years after irradiation in a light-water moderated PWR to $33 \mathrm{GWd} / \mathrm{t}$ burnup. The irradiated fuel contains $956 \mathrm{~kg} \mathrm{U}, 35 \mathrm{~kg}$ of $\mathrm{FPs}$, and $8.7 \mathrm{~kg}$ of $\mathrm{Pu}$, and 1.0 $\mathrm{kg}$ of other TRUs. The left charts break down the composition of the fuel on a molar basis, while the right-most chart illustrates the contributions of the different groups of elements to the radioactivity of the same fuel. (Courtesy of Colorado School of Mines. Based on data from Choppin et al. 2002.)

The elements that dominate the fuel on a molar or mass basis, which is an important consideration for separations, are less important to the radioactivity of irradiated fuel. Alkali and alkaline earth elements each comprise only $0.5 \mathrm{~mol} \%$ of the used fuel, but soon after irradiation stops, they come to dominate the 
radioactivity of the fuel for the next few centuries due to the radioactivity from the ${ }^{90} \mathrm{Sr} /{ }^{90} \mathrm{Y}$ and ${ }^{137} \mathrm{Cs} /{ }^{137 m} \mathrm{Ba}$ decay pairs. Ten years after removal from the reactor, the alkali and alkaline earth elements account for $75 \%$ of the radiation from representative commercial nuclear fuels (Figures A.1 and A.2). The other major contributor to the radioactivity of these fuels is ${ }^{241} \mathrm{Pu}(0.08 \mathrm{~mol} \%)$, which decays $\left(t_{1 / 2}=14.3\right.$ years $)$ to

${ }^{241} \mathrm{Am}$. Comparing Figure A.1 and Figure A.3, the effects of increased irradiation on the radioactivity of the fuel are clear. Higher burnup results from more fissions and more FPs, resulting in more radioactive fuel. Higher burnup also favors production of heavy $\mathrm{Pu}$ isotopes, such as ${ }^{241} \mathrm{Pu}$, which also increases the radioactivity in the $\mathrm{Pu}$ fraction of the fuel in the short term and the ${ }^{241} \mathrm{Am}$ content of the fuel in the long term if the $\mathrm{Pu}$ is not separated.
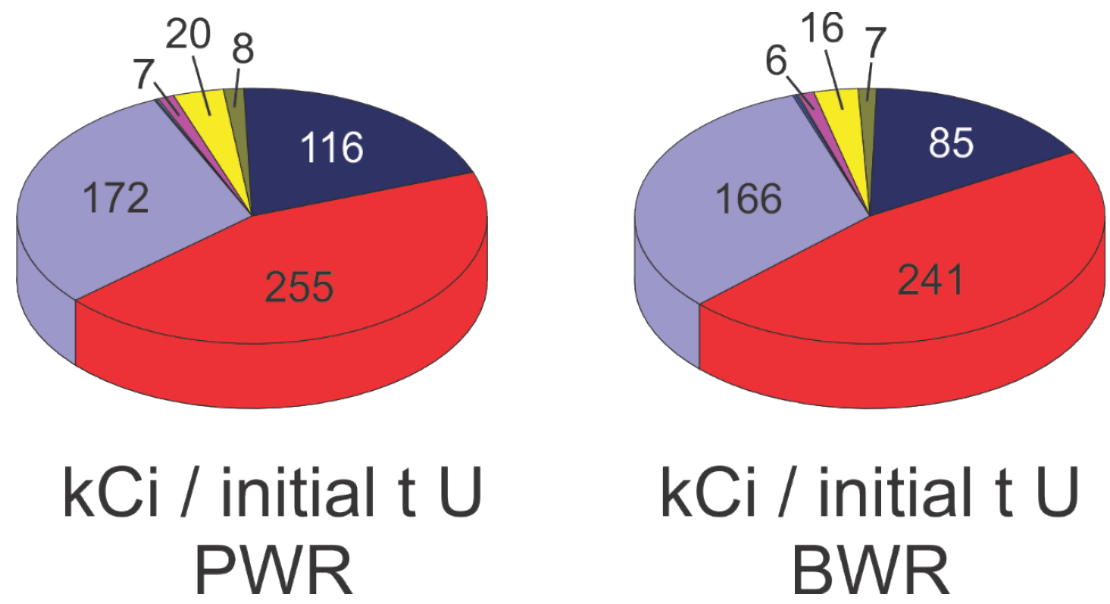

Figure A.2. Postirradiation radioactivity in a 1 ton low-enriched uranium dioxide fuel 10 years after irradiation in a light water moderated pressurized water reactor (PWR) or boiling water reactor (BWR). PWR: initial enrichment $4.2 \%$, burnup $50 \mathrm{GWd} / \mathrm{t}$; BWR: initial enrichment 4.0\%, burnup $49 \mathrm{GWd} / \mathrm{t}$. (Courtesy of Colorado School of Mines. Based on data from NRC 2007.)

Fuels designed for other types of nuclear reactors, such as natural $U$ (for non-light water moderated reactors), high-assay low-enriched $\mathrm{U}$ (HALEU), $\mathrm{U} / \mathrm{Pu}$ or $\mathrm{Th} / \mathrm{Pu} \mathrm{MOX}$, or $\mathrm{Th} /{ }^{23} \mathrm{U}$ fuels, will present different distributions of elements. While the creation of particular FPs might be enhanced or degraded with different fuels or different reactor types, the overall categories of FPs will remain. The lightest fissile nuclide, ${ }^{233} \mathrm{U}$, favors the production of ${ }^{90} \mathrm{Sr}$ and light transition metal $\mathrm{FPs}$ as compared to the data presented in Figures A.1 and A.2, while the heavier fissile nuclides $\left({ }^{239} \mathrm{Pu}\right.$ and $\left.{ }^{241} \mathrm{Pu}\right)$ favor production of the heavier $4 \mathrm{~d}$ transition metals (Tc-Pd) and lighter lanthanides FPs. Fuels with less ${ }^{238} \mathrm{U}$ generally produce less $\mathrm{Pu}$ and transplutonium elements, with $\mathrm{Th} /{ }^{233} \mathrm{U}$ fuels making much less $\mathrm{Pu}(10$ to $>100$ times less) than conventional LEU ${ }^{235} \mathrm{U} /{ }^{238} \mathrm{U}$ fuels, but more Pa will be produced and ${ }^{237} \mathrm{~Np}$ will become the most important minor actinide. In contrast, Pu-based fuels will favor production of transplutonium elements through neutron-capture reactions and beta decay, and this will be accentuated in closed fuel cycles that repeatedly recycle $\mathrm{Pu}$ and minor actinides (e.g., Am and $\mathrm{Cm}$ ). Furthermore, fast-spectrum reactors with higher fission:neutron capture ratios that disfavor production of $\mathrm{Pu}$ or transplutonium elements by neutron capture could be employed for future commercial reactor designs.

\section{References}

Choppin, G. R.; Liljenzin, J. O.; Rydberg, J. Radiochemistry and Nuclear Chemistry. ButterworthHeinemann: Woburn, MA, 2002.

NRC. Characteristics for the Representative Commercial Spent Fuel Assembly for Preclosure Normal Operations. Washington, DC: US Nuclear Regulatory Commission, 2007. 


\section{Appendix B. Example Fuel Cycle Options}

To provide context to the workshop participants, a plenary presentation was given describing potential fuel cycle options that would require implementation of separation science and technology. The fuel cycle options can be grouped into two categories:

1. Monorecycle in which actinides ( $\mathrm{U}, \mathrm{Pu}$, and perhaps $\mathrm{Np}$ ) are recovered, converted into fuel, and recycled one time into thermal power reactors

2. Multirecycle in which actinides (potentially $U$ through $\mathrm{Cm}$ ) are recovered and converted into fuels or targets for burning in a fleet of advanced reactors. In this case, the actinides are continually recycled into suitable reactors.

The monorecycle options can be implemented in light-water reactors (LWRs) such as those currently used in the nuclear power industry. The multirecycle options require a fleet of advanced reactors but are attractive in that the nuclear fuel cycle becomes nearly completely sustainable with minimal need for freshly mined U (NEA 2021).

Figure B.1 schematically shows an example fuel cycle scenario in which $\mathrm{U}$ and $\mathrm{Pu}$ are recycled a single time. In this scenario, fresh $U$ oxide fuel is burned in a light-water reactor (LWR). Upon discharge from the LWR, the fuel is processed to recover $\mathrm{U}$ and $\mathrm{Pu}$ as pure products. The plutonium uranium reduction extraction (PUREX) process is the currently used industrial process for recovering $\mathrm{U}$ and $\mathrm{Pu}$ from irradiated fuel (Herbst et al. 2011). The recovered $U$ is re-enriched to $3-5 \%{ }^{235} \mathrm{U}$, if necessary, and fabricated into $\mathrm{UO}_{2}$ fuel for recycle back into an LWR. The Pu is mixed with fresh $\mathrm{U}$ to make MOX fuel, which also is burned in a LWR. After irradiation the fuel containing the recycled U and the MOX fuel are disposed of in a geological repository.

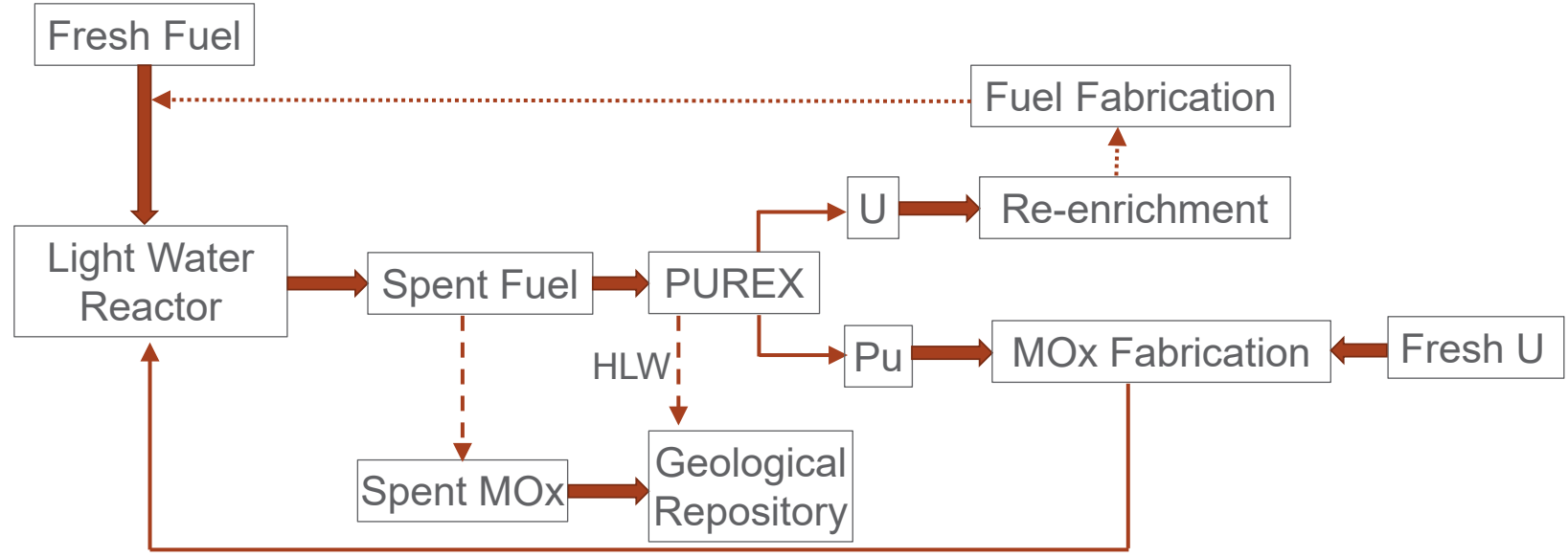

Figure B.1. Example fuel cycle scenario with monorecycle of $U$ and Pu. (Courtesy of PNNL.)

Figure B.2 presents an example fuel cycle scenario with multirecycle of $U$ and Pu. Again, fresh $U$ oxide fuel is burned in an LWR in this scenario. But in this case, the used fuel from the LWR is processed to recover the bulk $\mathrm{U}$ and a mixed $\mathrm{U} / \mathrm{Pu}$ product. Eliminating the separation of pure $\mathrm{Pu}$ is desirable from the standpoint of nuclear nonproliferation. These separations could be achieved through the Co-Extraction $\left(\right.$ COEX $\left.^{\mathrm{TM}}\right)$ (Drain et al. 2008), Advanced PUREX (Fox et al. 2006), or co-decontamination (Lumetta et al. 2019) processes. As in the example in Figure B.1, the recovered $U$ is re-enriched to $3-5 \%{ }^{235} \mathrm{U}$, if necessary, and fabricated into $\mathrm{UO}_{2}$ fuel for recycle back into an LWR. Alternatively, the $\mathrm{U}$ could go directly to fuel fabrication for a fast reactor. The mixed $\mathrm{U} / \mathrm{Pu}$ is fabricated into fast reactor fuel. Eventually, the $\mathrm{U}$ and $\mathrm{Pu}$ 
are continually separated and recycled into the fast reactor. All HLW generated during the actinide separation processes is immobilized and disposed of in a geological repository.

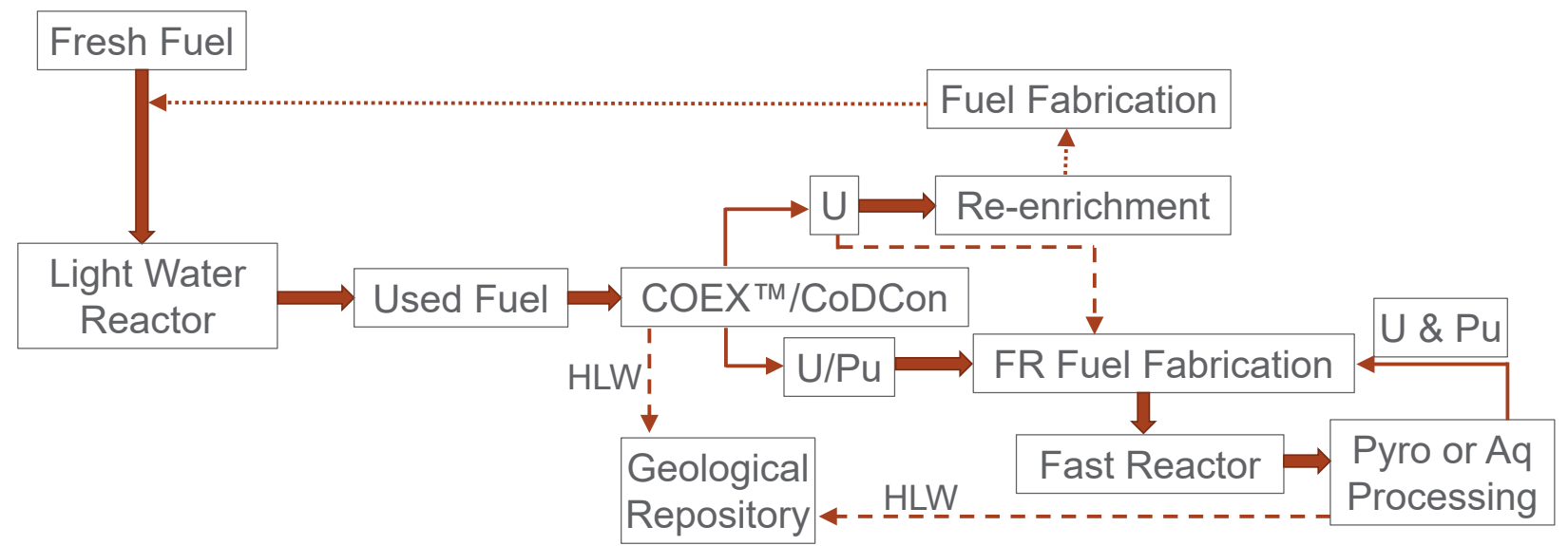

Figure B.2. Example fuel cycle scenario with multirecycle of $U$ and Pu. (Courtesy of PNNL.)

Table B.1 summarizes several fuel cycle options that can be considered. It should be kept in mind that some of the monorecycle scenarios can be considered as stepping stones to the more advance multirecycle options.

Table B.1. Summary of Fuel Cycle Options.

\footnotetext{
Single recycle of $U$ in thermal reactor

Single recycle of $\mathrm{Pu}$ as MOX fuel in thermal reactor

Single recycle of bulk $\mathrm{U}$ and $\mathrm{Pu}$ as MOX fuel in thermal reactor

Single recycle of $\mathrm{Pu}$ as MOX fuel in thermal reactor; no separated $\mathrm{Pu}$

Single recycle of bulk $\mathrm{U}$ and $\mathrm{Pu}$ as MOX fuel in thermal reactor; no separated $\mathrm{Pu}$

Multirecycle of $\mathrm{Pu}$ and some $\mathrm{U}$ in fast reactors

Multirecycle of $\mathrm{Pu}$ and some $\mathrm{U}$ in fast reactors plus single recycle of bulk $\mathrm{U}$

Multirecycle of $\mathrm{Pu}$ and $\mathrm{Np}$ and some $\mathrm{U}$ in fast reactors

Multirecycle of $\mathrm{Pu}$ and $\mathrm{Np}$ and some $\mathrm{U}$ in fast reactors plus single recycle of bulk $\mathrm{U}$

Multirecycle of $\mathrm{Pu}$ and $\mathrm{MA}$ and some $\mathrm{U}$ in fast reactors

Multirecycle of $\mathrm{Pu}$ and $\mathrm{MA}$ and some $\mathrm{U}$ in fast reactors plus single recycle of bulk $\mathrm{U}$
}

\section{References}

Drain, F.; Emin, J. L.; Vinoche, R.; Baron, P. COEX ${ }^{\mathrm{TM}}$ Process: Cross-Breeding between Innovation and Industrial Experience, 2008. WM2008. Phoenix, AZ: Paper 8220.

Fox, O. D.; Jones, C. J.; Birkett, J. E.; Carrott, M. J.; Crooks, G.; Maher, C. J.; Roube, C. V.; Taylor, R. J. Advanced PUREX Flowsheets for Future Np and Pu Fuel Cycle Demands. Separations for the Nuclear Fuel Cycle in the 21st Century. Lumetta, G. J., Nash, K. L., Clark, S. B., Friese, J. I. Washington, DC, 2006, American Chemical Society: 89-102.

Herbst, R. S.; Baron, P.; Nilsson, M. Standard and Advanced Separation: PUREX Processes for Nuclear Fuel Reprocessing. In Advanced Separation Techniques for Nuclear Fuel Reprocessing and 
Radioactive Waste Treatment. Nash, K. L., Lumetta, G. J. Oxford: Woodhead Publishing, 2011, pp 141-75.

Lumetta, G. J.; Allred, J. R.; Bryan, S. A.; Hall, G. B.; Levitskaia, T. G.; Lines, A. M.; Sinkov, S. I. Simulant Testing of a Co-Decontamination (CoDCon) Flowsheet for a Product with a Controlled Uranium-to-Plutonium Ratio. Separation Science and Technology 2019, 54: 1977-84.

NEA. Strategies and Considerations for the Back End of the Fuel Cycle. Nuclear Energy Agency, 2021. 


\section{Appendix C. Current State-of-the-Art: The PUREX Process}

All industrial plants for recycling of commercial power reactor fuel employ the PUREX process (Herbst et al. 2011). Although the specific flowsheets may vary from plant to plant (Swanson 1990), they all have the same basic features:

1. The fuel rods are chopped into small pieces $(2.5-7.5 \mathrm{~cm}$ long)

2. The irradiated fuel matrix is dissolved in hot $\mathrm{HNO}_{3}$

3. The dissolved fuel solution is clarified of undissolved solids

4. The dissolved fuel solution is adjusted to the appropriate $\mathrm{HNO}_{3}$ and $\mathrm{U}$ concentrations for feed to the solvent extraction circuit

5. Uranium and $\mathrm{Pu}$ are coextracted from the feed solution

6. Plutonium is stripped from the loaded solvent using a reducing agent

7. Uranium is stripped from the $\mathrm{Pu}$-depleted solvent with dilute $\mathrm{HNO}_{3}$

8. The stripped solvent is washed and conditioned, and then is recycled back to the extraction section

9. The $\mathrm{U}$ and $\mathrm{Pu}$ products are subjected to one or more subsequent purification cycles to achieve the desired purity of the products.

The state of knowledge regarding dissolution of $\mathrm{UO}_{2}$ in $\mathrm{HNO}_{3}$ has recently been reviewed (Marc et al. 2017). The dissolution reaction is believed to proceed predominantly according to the following two reactions.

$$
\begin{gathered}
\mathrm{UO}_{2}+8 /{ }_{3} \mathrm{HNO}_{3} \rightarrow \mathrm{UO}_{2}\left(\mathrm{NO}_{3}\right)_{2}+2 /{ }_{3} \mathrm{NO}+4 /{ }_{3} \mathrm{H}_{2} \mathrm{O} \\
\mathrm{UO}_{2}+4 \mathrm{HNO}_{3} \rightarrow \mathrm{UO}_{2}\left(\mathrm{NO}_{3}\right)_{2}+2 \mathrm{NO}_{2}+2 \mathrm{H}_{2} \mathrm{O}
\end{gathered}
$$

The $\mathrm{Pu}$ in the fuel matrix is essentially completely dissolved in $\mathrm{HNO}_{3}$ along with the $\mathrm{U}$, as are most of the $\mathrm{FP}$ elements. The undissolved solids are dominated by the elements $\mathrm{Mo}, \mathrm{Tc}, \mathrm{Ru}, \mathrm{Rh}$, and $\mathrm{Pd}(\mathrm{Buck}$ et al. 2015).

The heart of the PUREX process is the ability of TBP to extract $\mathrm{Pu}(\mathrm{IV})$ and U(VI) from $\mathrm{HNO}_{3}$ media. Figure C.1 shows the chemical structure of TBP and representative distribution ratio ${ }^{6}$ data for a typical PUREX system (Irish and Reas 1957). As can be seen from these data, U(VI) and Pu(IV) distribution ratios are orders-of-magnitude greater than those for most FPs. The dominant extraction reactions are believed to be those described by Eqs. 3 and 4 (Herbst et al. 2011), but recent results suggest that other species are likely involved (Lumetta et al. 2021).

$$
\begin{aligned}
& \mathrm{UO}_{2}{ }^{2+}(\mathrm{aq})+2 \mathrm{NO}_{3}{ }^{-}(\mathrm{aq})+2 \mathrm{TBP}(\mathrm{org}) \rightarrow \mathrm{UO}_{2}\left(\mathrm{NO}_{3}\right)_{2}(\mathrm{TBP})_{2}(\mathrm{org}) \\
& \mathrm{Pu}^{4+}(\mathrm{aq})+4 \mathrm{NO}_{3}{ }^{-}(\mathrm{aq})+2 \mathrm{TBP}(\text { org }) \rightarrow \mathrm{Pu}\left(\mathrm{NO}_{3}\right)_{4}(\mathrm{TBP})_{2}(\text { org })
\end{aligned}
$$

The trivalent actinides Am and Cm are poorly extracted by TBP and are easily routed to the HLW raffinate arising from the first extraction cycle. The behavior of $\mathrm{Np}$ is notoriously complex in the PUREX process because of its propensity to exist in oxidations states from +4 to +6 (Taylor et al. 2013). To a large extent, the differences between various PUREX flowsheets are based on different approaches to managing Np in the process. Technetium is another component that is challenging to manage in the process because of its

${ }^{6}$ The distribution ratio is defined as the concentration of the component in the organic phase divided by the concentration in the aqueous phase. 
coextraction with $\mathrm{U}(\mathrm{VI})$ and $\mathrm{Pu}(\mathrm{IV})$, which is exacerbated by the presence of $\mathrm{Zr}$ (Vialard and Germain 1986).

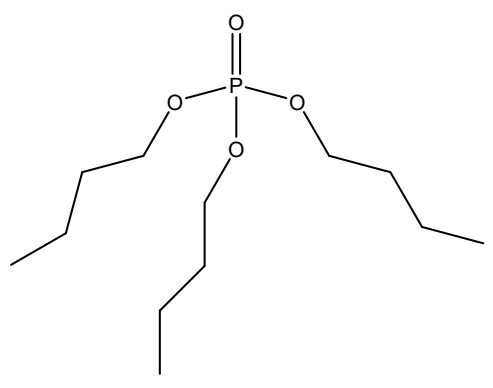

TBP

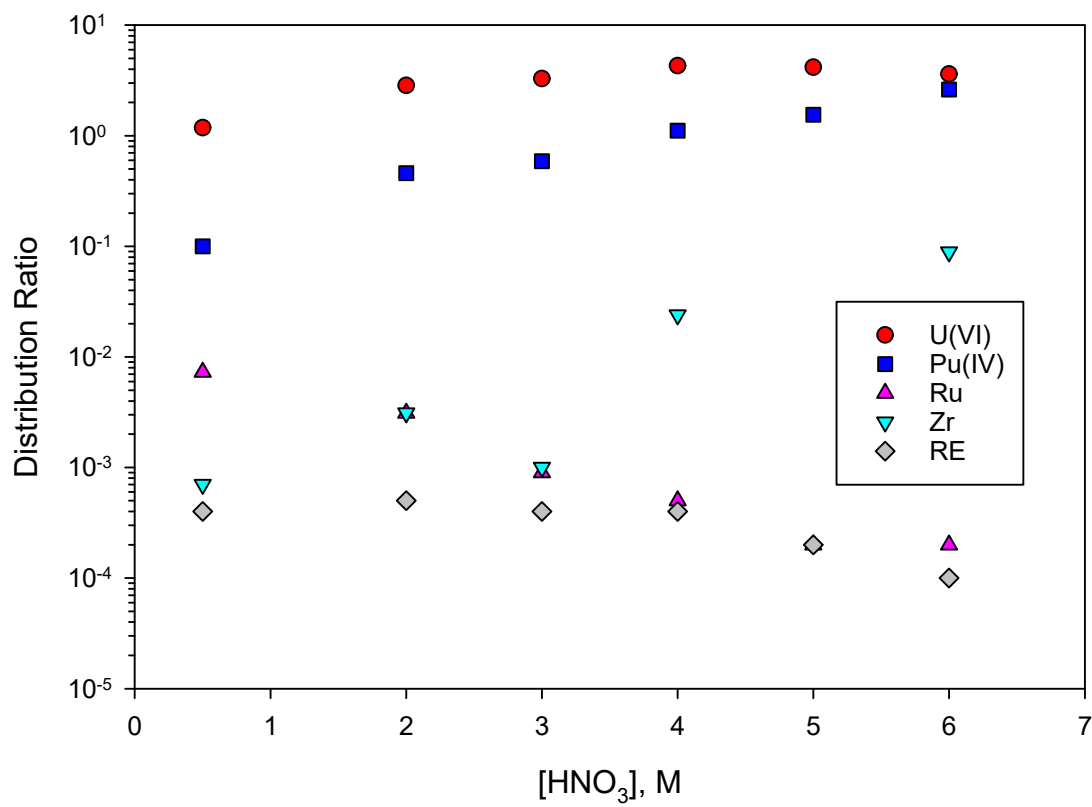

Figure C.1. Chemical structure of TBP (left); distribution ratios for selected fuel components in the PUREX system (solvent $30 \%$ TBP in an unspecified aliphatic diluent; initial aqueous $U$ concentration = $200 \mathrm{~g} / \mathrm{L}$; other components at tracer level; RE = rare earth elements). (Courtesy of PNNL. Data from Irish and Reas 1957.)

The chemical engineering principles for implementing the PUREX process are highly developed (Arm and Phillips 2011). The solvent extraction operations where there is substantial amounts of Pu present are typically performed in pulsed columns, which can be designed to be critically safe. Centrifugal contactors have been used for this purpose, but not as extensively as pulsed columns. However, there is renewed interest in centrifugal contactor technology, and these would likely be used in future fuel recycling facilities. Mixer-settlers are generally used where there are no criticality safety concerns such as in the U purification cycles. They are advantageous because of their mechanical simplicity.

\section{References}

Arm, S. T.; Phillips, C. Chemical Engineering for Advanced Aqueous Radioactive Materials Separations. Advanced Separation Techniques for Nuclear Fuel Reprocessing and Radioactive Waste Treatment. Nash, K. L.; Lumetta, G. J. Eds. Cambridge, Woodhead Publishing, 2011: 58-94.

Buck, E. C.; Mausolf, E. J.; McNamara, B. K.; Soderquist, C. Z.; Schwantes, J. M. Nanostructure of Metallic Particles in Light Water Reactor Used Nuclear Fuel. Journal of Nuclear Materials 2015, 461, 236-43.

Herbst, R. S.; Baron, P.; Nilsson, M. Standard and Advanced Separation: PUREX Processes for Nuclear Fuel Reprocessing. Advanced Separation Techniques for Nuclear Fuel Reprocessing and Radioactive Waste Treatment. Nash, K. L.; Lumetta, G. J. Oxford, Woodhead Publishing, 2011: $141-75$.

Irish, E. R.; Reas, W. H. The PUREX Process-A Solvent Extraction Reprocessing Method for Irradiated Uranium. General Electric Hanford Atomic Products Operation: Hanford, WA, 1957. 
Lumetta, G. J.; Heller, F. D.; Hall, G. B.; Asmussen, S. E.; Sinkov, S. I. Optical Spectroscopic Investigation of Hexavalent Actinide Ions in n-Dodecane Solutions of Tri-butyl Phosphate. Solvent Extraction and Ion Exchange 2021, 39, 56-73.

Marc, P.; Magnaldo, A.; Vaudano, A.; Delahaye, T.; Schaer, E. Dissolution of Uranium Dioxide in Nitric Acid Media: What Do We Know? EPJ Nuclear Sciences \& Technologies 2017, 3, 13.

Swanson, J. L. Purex Process Flowsheets. Science and Technology of Tributyl Phosphate. Schulz, W. W.; Burger, L. L.; Navratil J. D. Boca Raton: CRC Press, 1990. III, 55-79.

Taylor, R. J.; Gregson, C. R.; Carrott, M. J.; Mason, C.; Sarsfield, M. J. Progress Towards the Full Recovery of Neptunium in an Advanced PUREX Process. Solvent Extraction and Ion Exchange 2013, 31(4), 442-62.

Vialard, E.; Germain, M. Technetium Behaviour Control in the PUREX Process. International Solvent Extraction Conference, Munich, Germany, International Committee for Solvent Extraction Chemistry and Technology, 1986. 


\section{Appendix D. Workshop Organizing Committee}

\section{Chairs}

Bruce Moyer

moyerba@ornl.gov

Chair Oak Ridge National Laboratory

Gregg Lumetta

gregg.lumetta@pnnl.gov

Co-chair Pacific Northwest National Laboratory

Solid-Gas Separations Panel

Stephanie Bruffey

Lead Oak Ridge National Laboratory

bruffeysh@ornl.gov

Sarah Finkeldei

Co-lead University of California, Irvine

sfinkeld@uci.edu

Solid-Liquid Separations Panel

Ken Marsden

Lead Idaho National Laboratory

ken.marsden@inl.gov

Michael Simpson

Co-lead University of Utah

michael.simpson@utah.edu

Liquid-Liquid Separations Panel

Mark Jensen

Lead Colorado School of Mines

mjensen@mines.edu

Peter Zalupski

Co-lead

Idaho National Laboratory

peter.zalupski@inl.gov

Office of Nuclear Energy Lead

Stephen Kung

Director Office of Materials and Chemical Technologies stephen.kung@nuclear.energy.gov 


\section{Appendix E. Workshop Participants}

\begin{tabular}{|c|c|c|}
\hline Name & Institution & Email \\
\hline Abergel, Rebecca & $\begin{array}{l}\text { University of } \\
\text { California Berkeley } \\
\text { and Lawrence } \\
\text { Berkeley National } \\
\text { Laboratory }\end{array}$ & abergel@,berkeley.edu \\
\hline $\begin{array}{l}\text { Albrecht-Schönzart, } \\
\text { Thomas E. }\end{array}$ & $\begin{array}{l}\text { Florida State } \\
\text { University }\end{array}$ & albrecht-schmitt@chem.fsu.edu \\
\hline Alexandratos, Spiro & Hunter College & alexsd@hunter.cuny.edu \\
\hline Arm, Stuart & $\begin{array}{l}\text { Pacific Northwest } \\
\text { National Laboratory }\end{array}$ & stuart.arm@pnnl.gov \\
\hline Bader, Sven & Orano Group & sven.bader@orano.group \\
\hline Batista, Enrique & $\begin{array}{l}\text { Argonne National } \\
\text { Laboratory }\end{array}$ & erb@anl.gov \\
\hline Blakemore, James & University of Kansas & blakemore@ku.edu \\
\hline Boscoboinik, Jorge & $\begin{array}{l}\text { Brookhaven National } \\
\text { Laboratory }\end{array}$ & jboscoboinik@bnl.gov \\
\hline Britt, Phil & $\begin{array}{l}\text { Oak Ridge National } \\
\text { Laboratory }\end{array}$ & brittpf@ornl.gov \\
\hline Bruffey, Stephanie H. & $\begin{array}{l}\text { Oak Ridge National } \\
\text { Laboratory }\end{array}$ & bruffeysh@ornl.gov \\
\hline Brumaghim, Julia & Clemson University & brumagh@,clemson.edu \\
\hline $\begin{array}{l}\text { Bryantsev, } \\
\text { Vyacheslav }\end{array}$ & $\begin{array}{l}\text { Oak Ridge National } \\
\text { Laboratory }\end{array}$ & bryantsevv@ornl.gov \\
\hline Burns, Jon & $\begin{array}{l}\text { University of } \\
\text { Alabama }\end{array}$ & burnsjon@uab.edu \\
\hline Clark, Aurora & $\begin{array}{l}\text { Washington State } \\
\text { University }\end{array}$ & auclark@wsu.edu \\
\hline Collins, Emory & $\begin{array}{l}\text { Oak Ridge National } \\
\text { Laboratory }\end{array}$ & collinsed@ornl.gov \\
\hline Dai, Sheng & $\begin{array}{l}\text { Oak Ridge National } \\
\text { Laboratory }\end{array}$ & dais@ornl.gov \\
\hline Dares, Chris & $\begin{array}{l}\text { Florida International } \\
\text { University }\end{array}$ & cdares@fiu.edu \\
\hline Deblonde, Gauthier & $\begin{array}{l}\text { Lawrence Livermore } \\
\text { National Laboratory }\end{array}$ & deblonde1@,lnl.gov \\
\hline del Cul, Guillermo & $\begin{array}{l}\text { Oak Ridge National } \\
\text { Laboratory }\end{array}$ & delculgd@ornl.gov \\
\hline Delmau, Lætitia & $\begin{array}{l}\text { Oak Ridge National } \\
\text { Laboratory }\end{array}$ & delmaulh@ornl.gov \\
\hline DiBona, Kristin & Wyonics & krdibona@wyonics.com \\
\hline
\end{tabular}




\begin{tabular}{|c|c|c|}
\hline Name & Institution & Email \\
\hline Dixon, David & $\begin{array}{l}\text { University of } \\
\text { Alabama }\end{array}$ & dadixon@bama.ua.edu \\
\hline Ebert, William & $\begin{array}{l}\text { Argonne National } \\
\text { Laboratory }\end{array}$ & ebert@,Anl.gov \\
\hline Finkeldei, Sarah & $\begin{array}{l}\text { University of } \\
\text { California, Irvine }\end{array}$ & sfinkeld@uci.edu \\
\hline Flood, Amar & Indiana University & aflood@indiana.edu \\
\hline Francesconi, Lynn & Hunter College & 1frances@hunter.cuny.edu \\
\hline Gelis, Artem & $\begin{array}{l}\text { University of Nevada, } \\
\text { Las Vegas }\end{array}$ & artem.gelis@unlv.edu \\
\hline Ghaleb, Khalil & Orano Group & khalil.abbasghaleb@orano.group \\
\hline Gharibyan, Narek & $\begin{array}{l}\text { Argonne National } \\
\text { Laboratory }\end{array}$ & gharibyan1@1lnl.gov \\
\hline Gray, Kimberly & $\begin{array}{l}\text { Department of } \\
\text { Energy, Office of } \\
\text { Nuclear Energy }\end{array}$ & kimberly.gray@nuclear.energy.gov \\
\hline Griffith, Andrew & $\begin{array}{l}\text { Department of } \\
\text { Energy, Office of } \\
\text { Nuclear Energy }\end{array}$ & andrew.griffith@nuclear.energy.gov \\
\hline Grimes, Travis & $\begin{array}{l}\text { Idaho National } \\
\text { Laboratory }\end{array}$ & travis.grimes@,inl.gov \\
\hline Haghighat, Alireza & Virginia Tech & haghighat@,vt.edu \\
\hline Hall, Gabriel & $\begin{array}{l}\text { Pacific Northwest } \\
\text { National Laboratory }\end{array}$ & gabriel.hall@pnnl.gov \\
\hline Hawthorne, Krista & $\begin{array}{l}\text { Argonne National } \\
\text { Laboratory }\end{array}$ & khawthorne@anl.gov \\
\hline Hayton, Trevor & $\begin{array}{l}\text { University of } \\
\text { California, Santa } \\
\text { Barbara }\end{array}$ & hayton@chem.ucsb.edu \\
\hline Heathman, Colt & $\begin{array}{l}\text { Idaho National } \\
\text { Laboratory }\end{array}$ & colt.heathman@inl.gov \\
\hline Heres, Xavier & $\begin{array}{l}\text { Commissariat à } \\
\text { 1'Energie Atomique } \\
\text { (CEA), France }\end{array}$ & xavier.heres@cea.fr \\
\hline Herrmann, Steven & $\begin{array}{l}\text { Idaho National } \\
\text { Laboratory }\end{array}$ & steven.herrmann@inl.gov \\
\hline Hill, Caleb & $\begin{array}{l}\text { University of } \\
\text { Wyoming }\end{array}$ & caleb.hill@uwyo.edu \\
\hline Hin, Celine & Virginia Tech & celhin@vt.edu \\
\hline Holliday, Kiel & $\begin{array}{l}\text { Lawrence Livermore } \\
\text { National Laboratory }\end{array}$ & holliday7@1lnl.gov \\
\hline Horne, Gregory & $\begin{array}{l}\text { Idaho National } \\
\text { Laboratory }\end{array}$ & Gregory.Horne@,inl.gov \\
\hline
\end{tabular}




\begin{tabular}{|c|c|c|}
\hline Name & Institution & Email \\
\hline Hoyt, Nathaniel & $\begin{array}{l}\text { Argonne National } \\
\text { Laboratory }\end{array}$ & nhoyt@anl.gov \\
\hline Iizuka, Masatoshi & $\begin{array}{l}\text { Central Research } \\
\text { Institute of Electric } \\
\text { Power Industry } \\
\text { (CRIEPI), Japan }\end{array}$ & iizuka@criepi.denken.or.jp \\
\hline $\begin{array}{l}\text { Jansone-Popova, } \\
\text { Santa }\end{array}$ & $\begin{array}{l}\text { Oak Ridge National } \\
\text { Laboratory }\end{array}$ & jansonepopos@,ornl.gov \\
\hline Jensen, Mark & $\begin{array}{l}\text { Colorado School of } \\
\text { Mines }\end{array}$ & mjensen@mines.edu \\
\hline Johnson, Roland & Muons, Inc. & $\underline{\text { rol@muonsinc.com }}$ \\
\hline Kanatzidis, Mercouri & $\begin{array}{l}\text { Northwestern } \\
\text { University }\end{array}$ & m-kanatzidis@northwestern.edu \\
\hline Karay, Nicholas & $\begin{array}{l}\text { Savannah River } \\
\text { National Laboratory }\end{array}$ & Nicholas.Karay@srnl.doe.gov \\
\hline Kavallieratos, Kostas & $\begin{array}{l}\text { Florida International } \\
\text { University }\end{array}$ & kavallie@fiu.edu \\
\hline King, James & $\begin{array}{l}\text { Idaho National } \\
\text { Laboratory }\end{array}$ & james.king@inl.gov \\
\hline Knope, Karah & $\begin{array}{l}\text { Georgetown } \\
\text { University }\end{array}$ & $\underline{\text { Karah.Knope@georgetown.edu }}$ \\
\hline Kung, Stephen & $\begin{array}{l}\text { Department of } \\
\text { Energy, Office of } \\
\text { Nuclear Energy }\end{array}$ & stephen.kung@nuclear.energy.gov \\
\hline Ledoux, Bob & $\begin{array}{l}\text { Department of } \\
\text { Energy, ARPA-E }\end{array}$ & Robert.ledoux@hq.doe.gov \\
\hline Leggett, Christina & $\begin{array}{l}\text { Department of } \\
\text { Energy, ARPA-E }\end{array}$ & Christina.Leggett@hq.doe.gov \\
\hline Lesica, Susan & $\begin{array}{l}\text { Department of } \\
\text { Energy, Office of } \\
\text { Nuclear Energy }\end{array}$ & Sue.lesica@nuclear.energy.gov \\
\hline Li, Shelly & University of Utah & shelly.li@utah.edu \\
\hline Lines, Amanda & $\begin{array}{l}\text { Pacific Northwest } \\
\text { National Laboratory }\end{array}$ & amanda.lines@.pnnl.gov \\
\hline Lobo, JD & Mu*STAR & jdlobo@muonsinc.com \\
\hline Lumetta, Gregg J. & $\begin{array}{l}\text { Pacific Northwest } \\
\text { National Laboratory }\end{array}$ & gregg.lumetta@pnnl.gov \\
\hline Lyon, Kevin & $\begin{array}{l}\text { Idaho National } \\
\text { Laboratory }\end{array}$ & kevin.lyon@inl.gov \\
\hline Marsden, Ken C. & $\begin{array}{l}\text { Idaho National } \\
\text { Laboratory }\end{array}$ & ken.marsden@inl.gov \\
\hline Martin, Leigh & $\begin{array}{l}\text { Oak Ridge National } \\
\text { Laboratory }\end{array}$ & $\underline{\text { martInlr@ornl.gov }}$ \\
\hline
\end{tabular}




\begin{tabular}{|c|c|c|}
\hline Name & Institution & Email \\
\hline Matuszak, Daniel & $\begin{array}{l}\text { Department of } \\
\text { Energy, Office of } \\
\text { Science }\end{array}$ & daniel.matuszak@science.doe.gov \\
\hline Matyas, Josef & $\begin{array}{l}\text { Pacific Northwest } \\
\text { National Laboratory }\end{array}$ & josef.matyas@pnnl.gov \\
\hline Mayes, Richard & $\begin{array}{l}\text { Oak Ridge National } \\
\text { Laboratory }\end{array}$ & mayesrt@ornl.gov \\
\hline McCann, Kevin & $\begin{array}{l}\text { Savannah River } \\
\text { National Laboratory }\end{array}$ & kevin.mccann@srnl.doe.gov \\
\hline McNamara, Bruce & $\begin{array}{l}\text { Pacific Northwest } \\
\text { National Laboratory }\end{array}$ & bruce.mcnamara@pnnel.gov \\
\hline Moyer, Bruce A. & $\begin{array}{l}\text { Oak Ridge National } \\
\text { Laboratory }\end{array}$ & moyerba@ornl.gov \\
\hline Murakami, Tsuyoshi & $\begin{array}{l}\text { Central Research } \\
\text { Institute of Electric } \\
\text { Power Industry } \\
\text { (CRIEPI), Japan }\end{array}$ & m-tsuyo@criepi.denken.or.jp \\
\hline Nash, Kenneth L. & $\begin{array}{l}\text { Washington State } \\
\text { University }\end{array}$ & knash@,wsu.edu \\
\hline O'Hara, Matt & $\begin{array}{l}\text { Pacific Northwest } \\
\text { National Laboratory }\end{array}$ & matthew.ohara@pnnl.gov \\
\hline Parkison, Adam & $\begin{array}{l}\text { Oak Ridge National } \\
\text { Laboratory }\end{array}$ & parkisonaj@ornl.gov \\
\hline Pereira, Candido & $\begin{array}{l}\text { Argonne National } \\
\text { Laboratory }\end{array}$ & pereira@anl.gov \\
\hline Peterman, Dean & $\begin{array}{l}\text { Idaho National } \\
\text { Laboratory }\end{array}$ & dean.peterman@inl.gov \\
\hline $\begin{array}{l}\text { Phongikaroon, } \\
\text { Supathorn }\end{array}$ & $\begin{array}{l}\text { Virginia } \\
\text { Commonwealth } \\
\text { University }\end{array}$ & sphongikaroon@vcu.edu \\
\hline Pierce, Bob & $\begin{array}{l}\text { Savannah River } \\
\text { National Laboratory }\end{array}$ & robert.pierce@srnl.doe.gov \\
\hline Powell, Brian A. & Clemson University & bpowell@,clemson.edu \\
\hline Rappleye, Devin & $\begin{array}{l}\text { Brigham Young } \\
\text { University }\end{array}$ & devin_rappleye@byu.edu \\
\hline Richards, Jason & $\begin{array}{l}\text { Oak Ridge National } \\
\text { Laboratory }\end{array}$ & richardsjm@ornl.gov \\
\hline Rogers, Robin & $\begin{array}{l}\text { University of } \\
\text { Alabama/525 } \\
\text { Solutions }\end{array}$ & rdrogers@ua.edu \\
\hline Rudisill, Tracy S. & $\begin{array}{l}\text { Savannah River } \\
\text { National Laboratory }\end{array}$ & tracy.rudisill@srnl.doe.gov \\
\hline Schindelholz, Mara & $\begin{array}{l}\text { Sandia National } \\
\text { Laboratory }\end{array}$ & meschin@sandia.gov \\
\hline
\end{tabular}




\begin{tabular}{|c|c|c|}
\hline Name & Institution & Email \\
\hline Shafer, Jenifer & $\begin{array}{l}\text { Colorado School of } \\
\text { Mines and DOE } \\
\text { ARPA-E }\end{array}$ & jenifer.shafer@hq.doe.gov \\
\hline Shehee, Thomas & $\begin{array}{l}\text { Savannah River } \\
\text { National Laboratory }\end{array}$ & thomas.shehee@srnl.doe.gov \\
\hline Simpson, Michael & University of Utah & michael.simpson@utah.edu \\
\hline Sorel, Christian & $\begin{array}{l}\text { Commissariat à } \\
\text { l'Energie Atomique } \\
\text { (CEA), France }\end{array}$ & christian.sorel@cea.fr \\
\hline Taylor, Robin & $\begin{array}{l}\text { National Nuclear } \\
\text { Laboratory, United } \\
\text { Kingdom }\end{array}$ & robin.j.taylor@nnl.co.uk \\
\hline Thallapally, Praveen & $\begin{array}{l}\text { Pacific Northwest } \\
\text { National Laboratory }\end{array}$ & praveen.thallapally@pnnl.gov \\
\hline Tkac, Peter & $\begin{array}{l}\text { Argonne National } \\
\text { Laboratory }\end{array}$ & tkac@anl.gov \\
\hline Todd, Terry A. & $\begin{array}{l}\text { Idaho National } \\
\text { Laboratory }\end{array}$ & Terry.Todd@inl.gov \\
\hline Uysal, Ahmet & $\begin{array}{l}\text { Argonne National } \\
\text { Laboratory }\end{array}$ & ahmet@anl.gov \\
\hline Vienna, John & $\begin{array}{l}\text { Pacific Northwest } \\
\text { National Laboratory }\end{array}$ & john.vienna@pnnl.gov \\
\hline Vogelaar, R. Bruce & Virginia Tech & vogelaar@vt.edu \\
\hline Wai, Chien & $\begin{array}{l}\text { University of } \\
\text { Idaho/LCW } \\
\text { Supercritical } \\
\text { Technologies Corp }\end{array}$ & cwai@uidaho.edu \\
\hline Wetzler, Modi & Clemson University & mwetzle@clemson.edu \\
\hline Wilk, Philip & $\begin{array}{l}\text { Department of } \\
\text { Energy, Office of } \\
\text { Science }\end{array}$ & philip.wilk@science.doe.gov \\
\hline Willet, James & $\begin{array}{l}\text { Argonne National } \\
\text { Laboratory }\end{array}$ & willit@anl.gov \\
\hline Williamson, Mark A. & $\begin{array}{l}\text { Argonne National } \\
\text { Laboratory }\end{array}$ & williamson@anl.gov \\
\hline Wishart, Jim & $\begin{array}{l}\text { Brookhaven National } \\
\text { Laboratory }\end{array}$ & wishart@,bnl.gov \\
\hline Yang, Ping & $\begin{array}{l}\text { Los Alamos National } \\
\text { Laboratory }\end{array}$ & pyang@lanl.gov \\
\hline Zalupski, Peter & $\begin{array}{l}\text { Idaho National } \\
\text { Laboratory }\end{array}$ & peter.zalupski@inl.gov \\
\hline
\end{tabular}




\section{Appendix F. Workshop Agenda}

Monday, August 30, 2021

\begin{tabular}{|c|c|c|c|}
\hline \multicolumn{2}{|c|}{ Time (EDT) } & \multirow{2}{*}{$\begin{array}{l}\text { Topic } \\
\text { Plan for the day: Panelists } \\
\text { present novel concepts, } \\
\text { bringing out the nature of } \\
\text { each concept, how it } \\
\text { applies to nuclear fuel } \\
\text { cycles, and its advantages. }\end{array}$} & \multirow{2}{*}{$\begin{array}{l}\text { Speaker/moderator } \\
\text { Stephen Kung, Bruce Moyer, Gregg } \\
\text { Lumetta }\end{array}$} \\
\hline 10:00 & Plenary & & \\
\hline $10: 20$ & Plenary & $\begin{array}{l}\text { NE Introduction: Medium- } \\
\text { and Long-Term Goals }\end{array}$ & Andrew Griffith \\
\hline $10: 50$ & Plenary & $\begin{array}{l}\text { Fuel-Cycle Options and } \\
\text { Role of Separations }\end{array}$ & Gregg Lumetta \\
\hline $11: 30$ & Plenary & $\begin{array}{l}\text { Research Problems in } \\
\text { Process Implementation }\end{array}$ & Robin Taylor \\
\hline $12: 10$ & Break & & \\
\hline $12: 20$ & Breakout 1 & Solid-Gas Separations & Stephanie Bruffey, Sarah Finkeldei \\
\hline $12: 20$ & Breakout 2 & Solid-Liquid Separations & Ken Marsden, Michael Simpson \\
\hline $12: 20$ & Breakout 3 & Liquid-Liquid Separations & Mark Jensen, Peter Zalupski \\
\hline 13:10 & Break & & \\
\hline $13: 20$ & Plenary & $\begin{array}{l}\text { Waste Disposition and } \\
\text { Implications for } \\
\text { Separation Needs }\end{array}$ & John Vienna \\
\hline $14: 00$ & Plenary & $\begin{array}{l}\text { Summary of the } \\
\text { Conclusions of the ARPA- } \\
\text { E CURIE Workshop }\end{array}$ & Jenifer Shafer \\
\hline
\end{tabular}

14:40 Break

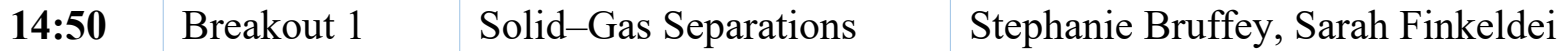

14:50 Breakout 2 Solid-Liquid Separations $\quad$ Ken Marsden, Michael Simpson 
\begin{tabular}{l|l|l|l} 
14:50 & Breakout 3 & Liquid-Liquid Separations & Mark Jensen, Peter Zalupski
\end{tabular}

\begin{tabular}{l|l|l|l}
$\mathbf{1 6 : 0 0}$ & Break & & \\
$\mathbf{1 6 : 1 0}$ & Plenary & $\begin{array}{l}\text { Report out from breakout } \\
\text { sessions }\end{array}$ & Panel Leads \\
\hline
\end{tabular}

16:30 Adjourn

Tuesday, August 31, 2021

\begin{tabular}{|c|c|c|c|}
\hline \multicolumn{2}{|c|}{ Time (EDT) } & \multirow{2}{*}{$\begin{array}{l}\text { Topic } \\
\text { Plan for the day: Panelists } \\
\text { evaluate the benefits and } \\
\text { risks of concepts, distill } \\
\text { knowledge and technology } \\
\text { gaps. }\end{array}$} & \multirow{2}{*}{$\begin{array}{l}\text { Speaker/moderator } \\
\text { Stephen Kung, Bruce Moyer, Gregg } \\
\text { Lumetta }\end{array}$} \\
\hline 10:00 & Plenary & & \\
\hline $10: 20$ & Plenary & $\begin{array}{l}\text { Challenges in Taking } \\
\text { Pyrochemical Concepts to } \\
\text { Deployment }\end{array}$ & Michael Simpson \\
\hline 11:00 & Break & & \\
\hline 11:10 & Breakout 1 & Solid-Gas Separations & Stephanie Bruffey, Sarah Finkeldei \\
\hline 11:10 & Breakout 2 & Solid-Liquid Separations & Ken Marsden, Michael Simpson \\
\hline 11:10 & Breakout 3 & Liquid-Liquid Separations & Mark Jensen, Peter Zalupski \\
\hline 13:00 & Break & & \\
\hline $13: 10$ & Breakout 1 & Solid-Gas Separations & Stephanie Bruffey, Sarah Finkeldei \\
\hline 13:10 & Breakout 2 & Solid-Liquid Separations & Ken Marsden, Michael Simpson \\
\hline $13: 10$ & Breakout 3 & Liquid-Liquid Separations & Mark Jensen, Peter Zalupski \\
\hline $15: 00$ & Break & & \\
\hline $15: 10$ & Plenary & $\begin{array}{l}\text { Report out from breakout } \\
\text { sessions }\end{array}$ & Panel Leads/Co-leads \\
\hline $15: 40$ & Plenary & Discussion & All \\
\hline $16: 30$ & Adjourn & & \\
\hline
\end{tabular}


Wednesday, September 1, 2021

\begin{tabular}{|c|c|c|c|}
\hline \multicolumn{2}{|c|}{ Time (EDT) } & Topic & Speaker/moderator \\
\hline 10:00 & Plenary & $\begin{array}{l}\text { Plan for the day: Panelists } \\
\text { identify and categorize } \\
\text { research needs. Group } \\
\text { collectively drafts target } \\
\text { future research directions } \\
\text { (FRDs) }\end{array}$ & $\begin{array}{l}\text { Stephen Kung, Bruce Moyer, Gregg } \\
\text { Lumetta }\end{array}$ \\
\hline $10: 20$ & Breakout 1 & Solid-Gas Separations & Stephanie Bruffey, Sarah Finkeldei \\
\hline $10: 20$ & Breakout 2 & Solid-Liquid Separations & Ken Marsden, Michael Simpson \\
\hline $10: 20$ & Breakout 3 & Liquid-Liquid Separations & Mark Jensen, Peter Zalupski \\
\hline $11: 50$ & Break & & \\
\hline $12: 10$ & Plenary & $\begin{array}{l}\text { Report out from breakout } \\
\text { sessions with key } \\
\text { knowledge/technology } \\
\text { gaps }\end{array}$ & Panel Leads \\
\hline $12: 40$ & Plenary & Discussion: Draft FRDs & All \\
\hline $13: 40$ & Break & & \\
\hline $14: 00$ & Plenary & Discussion: Finalize FRDs & All \\
\hline $15: 00$ & Plenary & Final remarks & $\begin{array}{l}\text { Stephen Kung, Bruce Moyer, Gregg } \\
\text { Lumetta }\end{array}$ \\
\hline
\end{tabular}




\section{Appendix G. White Papers}

\section{Liquid-Liquid Panel:}

\begin{tabular}{|c|c|}
\hline Author Name & White Paper Title \\
\hline Artem Gelis & $\begin{array}{l}\text { Single Solvent Extraction Process for U/TRU Recovery and Recycling } \\
\text { from Used Nuclear Fuel }\end{array}$ \\
\hline Colt Heathman & $\begin{array}{l}\text { Developing Two-Phase Separation Techniques for Advanced Fuel } \\
\text { Cycle Studies }\end{array}$ \\
\hline Amanda Lines & $\begin{array}{l}\text { Real-time process Monitoring and Automated Control to Enable } \\
\text { Economical and Proliferation Resistant Advanced Fuel Cycles }\end{array}$ \\
\hline Aurora Clark & The Control or Chaos Paradox \\
\hline Ahmet Uysal & Interfaces in Liquid-Liquid Extraction \\
\hline Amar Flood & From Third-Phase Mixtures to Ion-Driven Soft-Matter Separations \\
\hline Caleb Hill & $\begin{array}{l}\text { Novel Electrochemical Approaches to Separations for Nuclear Fuel } \\
\text { Reprocessing }\end{array}$ \\
\hline Peter Zalupski & Mining ${ }^{90} \mathrm{Sr}$ from Used Nuclear Fuel in Support of ${ }^{90} \mathrm{Sr} /{ }^{90} \mathrm{Y}$ Generator. \\
\hline Emory Collins & $\begin{array}{l}\text { Minor Actinide Partitioning and Transmutation Using Actual Actinide } \\
\text { Targets }\end{array}$ \\
\hline Gregory Horne & $\begin{array}{l}\text { Modeling the Fundamental Radiation Chemistry of the Organic } \\
\text { Diluent, and the Effect of Metal Ion Complexation on the } \\
\text { Radiochemical Behavior of Active Compounds }\end{array}$ \\
\hline Dean Peterman & $\begin{array}{l}\text { Impacts of Gamma Radiolysis on Tc Partitioning in Advanced } \\
\text { Aqueous Recycle of Used Nuclear Fuel }\end{array}$ \\
\hline Ping Yang & Data-Science for Separation \\
\hline $\begin{array}{l}\text { Vyacheslav } \\
\text { Bryantsev }\end{array}$ & $\begin{array}{l}\text { Computational Design and Screening on Novel Complexants for } \\
\text { Recycle of Used Nuclear Fuel }\end{array}$ \\
\hline David Dixon & Computational Approaches for the Design of Separation Systems \\
\hline Laetitia Delmau & $\begin{array}{l}\text { Adjacent Trivalent Actinides and Lanthanides Separation by Organic } \\
\text { Ligand/Aqueous Complexant System Optimization }\end{array}$ \\
\hline Robin Rogers & $\begin{array}{l}\text { Taking Advantage of the Unique Features of Ionic Liquids to Replace } \\
\text { the PUREX Process }\end{array}$ \\
\hline Amar Flood & Salt-selective Receptors for Extraction \\
\hline $\begin{array}{l}\text { Santa Jansone- } \\
\text { Popova }\end{array}$ & Innovative Approaches in Used Nuclear Fuel Reprocessing \\
\hline $\begin{array}{l}\text { Thomas E. } \\
\text { Albrecht-Schönzart }\end{array}$ & $\begin{array}{l}\text { Hyperpolarizability: A Quantifiable Property for Improved Recycling } \\
\text { in Closed Nuclear Fuel Cycles }\end{array}$ \\
\hline James Blakemore & $\begin{array}{l}\text { Strategic Modulation of Actinide Reduction Potentials for Improving } \\
\text { Recovery in Separations }\end{array}$ \\
\hline Xavier Heres & Plutonium Increase in Future Nuclear Fuel \\
\hline Julia Brumaghim & $\begin{array}{l}\text { Highly Covalent, Radiolytically Stable Complexants with Fast Kinetics } \\
\text { for Trivalent Separations }\end{array}$ \\
\hline Rebecca Abergel & $\begin{array}{l}\text { Chelation-Driven Redox Control for Actinide and Fission Product } \\
\text { Recovery }\end{array}$ \\
\hline
\end{tabular}


Kostas Soft donor ligands for Actinide/Lanthanide and Group Actinide

Kavallieratos Separations

\section{Solid-Liquid Panel:}

\section{Author Name White Paper Title}

Spiro Alexandratos Ion Exchange Polymers Applied to the Nuclear Fuel Cycle

Nicholas Karay PolyHIPE Foams for Efficient Separations

Thomas Shehee On-demand Capabilities for Nuclear Processing Using Additive

Manufacturing

Kevin McCann $\quad$ Reprocessing Nuclear Fuels Using Continuous Chromatographic

Separations

Gauthier Deblonde Fast, Efficient, Low-Cost, and Deployable Kit for Radioisotope

Capture and Purification

Jim Wishart Development of Robust and Effective Polyelectrolyte Membranes for

Efficient Lanthanide-Actinide Separation

Kristin Di Bona Implementation of Membrane Separations for Used Nuclear Fuel and

High Level Waste

Peter Tkac Lightening the Load on Solvent Extraction to Recycle Used Nuclear

Fuel

Jon Burns $\quad$ Sequestration of Used Nuclear Waste Stream Raffinates Using Pillared

Metal(IV) Phosphate-Phosphonate Hybrid Ion Exchange Materials and

Conversion to Final Ceramic Waste Form

\begin{tabular}{|c|c|}
\hline $\begin{array}{l}\text { Mercouri } \\
\text { Kanatzidis }\end{array}$ & Uranium Recovery from Used Nuclear Fuel Using Metal Sulfides \\
\hline Trevor Hayton & Chemical and Electrochemical Manipulation of the Uranyl Ion \\
\hline Chris Dares & Actinide Separation \\
\hline Jon Burns & $\begin{array}{l}\text { Group Hexavalent Actinide Separation by Co-Crystallization: A } \\
\text { Single-Step, Proliferation Resistant Approach to Nuclear Fuel Recycle }\end{array}$ \\
\hline $\begin{array}{l}\text { Supathorn } \\
\text { Phongikaroon }\end{array}$ & $\begin{array}{l}\text { Optimization and Assessment of a Hybrid Separation Method of Used } \\
\text { Nuclear Fuel for Advancement of Fuel Cycle Technology }\end{array}$ \\
\hline Bob Pierce & Uranium Metal from the Reduction of Purified Uranium Oxide \\
\hline Roland Johnson & $\begin{array}{l}\text { Continuous Removal of Fission Products from Molten-Salt Fueled } \\
\text { Subcritical Reactors }\end{array}$ \\
\hline Bruce McNamara & $\begin{array}{l}\text { Towards Automated, Continuous, Intra-Core Fission and Corrosion } \\
\text { Product Management in Molten Salt }\end{array}$ \\
\hline Steven Herrmann & Actinide-Lanthanide Separations in Pyrochemical Systems \\
\hline Tsuyshi Murakami & $\begin{array}{l}\text { Rare Earth Fission Products Separation from Used LiCl-KCl Melt by } \\
\text { Si Cathode for Reducing a Volume of the Salt Waste Form in } \\
\text { Pyroprocessing }\end{array}$ \\
\hline Michael Simpson & Minimal Salt Separations for Pyroprocessing \\
\hline $\begin{array}{l}\text { Mark A. } \\
\text { Williamson }\end{array}$ & $\begin{array}{l}\text { Fundamental Electrochemical Data Needs for Pyroprocess } \\
\text { Optimization }\end{array}$ \\
\hline James Willit & Reproducible Reference Electrodes for Molten Salts \\
\hline
\end{tabular}




\section{Solid-Gas Panel:}

\begin{tabular}{|c|c|}
\hline Author Name & White Paper Title \\
\hline Travis Grimes & $\begin{array}{l}\text { The Use of Acoustic Cavitation to Access HALEU from TRISO Fuel } \\
\text { Particles }\end{array}$ \\
\hline Gabriel Hall & Extraction of Actinides with Monoamides in Supercritical Fluids \\
\hline Chien Wai & Supercritical Fluid Extraction for Nuclear Fuel Cycle Application \\
\hline Gabriel Hall & $\begin{array}{l}\text { Two-step Proliferation Resistant, Enriched Uranium Recovery from } \\
\text { TRISO Fuel Types with Low Carbon Emission }\end{array}$ \\
\hline Jason Richards & Selective Fluoride Volatility Processing of UNF \\
\hline Emory Collins & Purification of Zirconium from Used Fuel Cladding \\
\hline Devin Rappleye & $\begin{array}{l}\text { Gas-phase Separation of Actinides Chlorides from Used Nuclear Fuels } \\
\text { and Process Salts }\end{array}$ \\
\hline Emory Collins & Addition of a Potential and Critical Benefit to SNF Reprocessing \\
\hline Stuart Arm & $\begin{array}{l}\text { Fissile Material Recovery Integrated Flowsheet Development for Waste } \\
\text { Reduction-Iodine and Tritium Management }\end{array}$ \\
\hline Tracy S. Rudisill & $\begin{array}{l}\text { Dissolution of Used Nuclear Fuel Using a Tributyl Phosphate } / n \text {-Paraffin } \\
\text { Solvent }\end{array}$ \\
\hline Gregg J. Lumetta & Alkaline-Side Processing of Used Nuclear Fuel \\
\hline Josef Matyas & Advancements in Sorption Technologies for Nuclear Applications \\
\hline $\begin{array}{l}\text { Praveen } \\
\text { Thallapally }\end{array}$ & $\begin{array}{l}\text { Tuning the Responsive Interactions with Noble Gases Using MOFs as } \\
\text { Molecular Switches }\end{array}$ \\
\hline Mara Schindelholz & $\begin{array}{l}\text { Embedded Sensors for In-situ Detection and Monitoring in Advanced } \\
\text { Nuclear Reactors }\end{array}$ \\
\hline
\end{tabular}




\section{Appendix H. Chapter Authors}

\begin{tabular}{|c|c|c|c|}
\hline Chapter & Title & Lead & Co-writers \\
\hline 1 & Introduction & $\begin{array}{l}\text { Gregg Lumetta } \\
\text { Pacific Northwest National } \\
\text { Laboratory }\end{array}$ & $\begin{array}{l}\text { Bruce Moyer } \\
\text { Oak Ridge National Laboratory }\end{array}$ \\
\hline 2 & $\begin{array}{l}\text { FRD 1: Develop } \\
\text { Methods to } \\
\text { Efficiently Remove } \\
\text { and Treat Coating } \\
\text { and Cladding } \\
\text { Materials }\end{array}$ & $\begin{array}{l}\text { Sarah Finkeldei } \\
\text { University of California, } \\
\text { Irvine }\end{array}$ & $\begin{array}{l}\text { Travis Grimes } \\
\text { Idaho National Laboratory } \\
\text { Gabriel Hall } \\
\text { Pacific Northwest National } \\
\text { Laboratory } \\
\text { Emory Collins } \\
\text { Oak Ridge National Laboratory } \\
\text { Stephanie Bruffey } \\
\text { Oak Ridge National Laboratory }\end{array}$ \\
\hline 3 & $\begin{array}{l}\text { FRD 2: Devise } \\
\text { Chemistry that } \\
\text { Operates Directly } \\
\text { on Irradiated Fuel }\end{array}$ & $\begin{array}{l}\text { Gregg Lumetta } \\
\text { Pacific Northwest National } \\
\text { Laboratory }\end{array}$ & $\begin{array}{l}\text { Gabriel Hall } \\
\text { Pacific Northwest National } \\
\text { Laboratory } \\
\text { Devin Rappleye } \\
\text { Brigham Young University } \\
\text { Bruce McNamara } \\
\text { Pacific Northwest National } \\
\text { Laboratory } \\
\text { Stuart Arm } \\
\text { Pacific Northwest National } \\
\text { Laboratory }\end{array}$ \\
\hline 4 & $\begin{array}{l}\text { FRD 3: Design } \\
\text { Robust Materials } \\
\text { for Separation of } \\
\text { Gas-Phase Species }\end{array}$ & $\begin{array}{l}\text { Stephanie Bruffey } \\
\text { Oak Ridge National } \\
\text { Laboratory }\end{array}$ & $\begin{array}{l}\text { Praveen Thallapally } \\
\text { Pacific Northwest National } \\
\text { Laboratory } \\
\text { Josef Matyas } \\
\text { Pacific Northwest National } \\
\text { Laboratory }\end{array}$ \\
\hline 5 & $\begin{array}{l}\text { FRD 4: Apply } \\
\text { Physical } \\
\text { Phenomena, Fields, } \\
\text { and Gradients to } \\
\text { Intensify } \\
\text { Separations }\end{array}$ & $\begin{array}{l}\text { Mark Jensen } \\
\text { Colorado School of Mines }\end{array}$ & \\
\hline 6 & $\begin{array}{l}\text { FRD 5: Exploit } \\
\text { Principles of } \\
\text { Coordination } \\
\text { Chemistry to } \\
\text { Simplify Actinide } \\
\text { Separations }\end{array}$ & $\begin{array}{l}\text { Peter Zalupski } \\
\text { Idaho National Laboratory }\end{array}$ & $\begin{array}{l}\text { Gregory Horne } \\
\text { Idaho National Laboratory } \\
\text { Ping Yang } \\
\text { Los Alamos National } \\
\text { Laboratory } \\
\text { Amar Flood } \\
\text { Indiana University }\end{array}$ \\
\hline
\end{tabular}




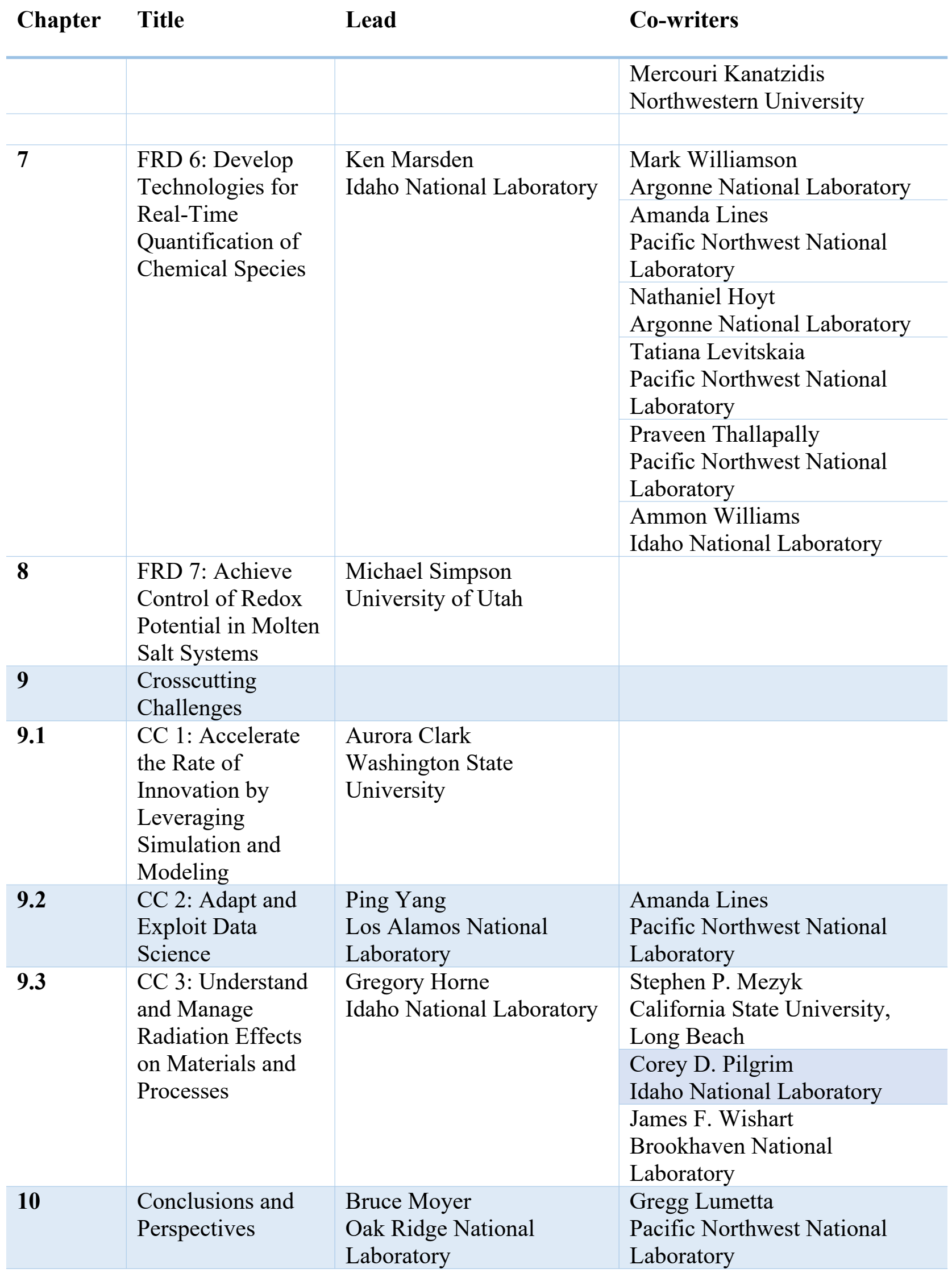




\section{$\begin{array}{lll}\text { Chapter Title Lead } & \text { Co-writers }\end{array}$}

\begin{tabular}{l|l|l|}
\hline $\begin{array}{l}\text { Appendix } \\
\text { A }\end{array}$ & $\begin{array}{l}\text { Composition of } \\
\text { Irradiated Fuel }\end{array}$ & $\begin{array}{l}\text { Mark Jensen } \\
\text { Colorado School of Mines }\end{array}$ \\
\hline $\begin{array}{l}\text { Appendix } \\
\text { B }\end{array}$ & $\begin{array}{l}\text { Example Fuel } \\
\text { Cycle Options }\end{array}$ & $\begin{array}{l}\text { Gregg Lumetta } \\
\text { Pacific Northwest National } \\
\text { Laboratory }\end{array}$ \\
\hline $\begin{array}{l}\text { Appendix } \\
\text { C }\end{array}$ & $\begin{array}{l}\text { Current State-of- } \\
\text { the-Art: The }\end{array}$ & $\begin{array}{l}\text { Gregg Lumetta } \\
\text { Pacific Northwest National }\end{array}$ \\
\hline
\end{tabular}


Ther 